\title{
Ultracold atomic gases in optical lattices: Mimicking condensed matter physics and beyond
}

\author{
Maciej Lewenstein, \\ ICREA and ICFO-Institut de Ciències Fotòniques, E-08660 Castelldefels (Barcelona) Spain \\ Anna Sanpera, \\ ICREA and Grup de Física Teòrica, Universitat Autònoma de Barcelona, E-08193 Bellaterra, Spain
}

Veronica Ahufinger,

ICREA and Grup d'Optica, Universitat Autònoma de Barcelona, E-08193 Bellaterra, Spain

Bogdan Damski,

Theory Division, Los Alamos National Laboratory, MS-B213, Los Alamos, NM 87545, USA

Aditi Sen(De), and Ujjwal Sen

ICFO-Institut de Ciències Fotòniques, E-08660 Castelldefels (Barcelona) Spain

(Received 00 Month 200x; In final form 00 Month 200x)

We review recent developments in the physics of ultracold atomic and molecular gases in optical lattices. Such systems are nearly perfect realisations of various kinds of Hubbard models, and as such may very well serve to mimic condensed matter phenomena. We show how these systems may be employed as quantum simulators to answer some challenging open questions of condensed matter, and even high energy physics. After a short presentation of the models and the methods of treatment of such systems, we discuss in detail, which challenges of condensed matter physics can be addressed with (i) disordered ultracold lattice gases, (ii) frustrated ultracold gases, (iii) spinor lattice gases, (iv) lattice gases in "artificial" magnetic fields, and, last but not least, (v) quantum information processing in lattice gases. For completeness, also some recent progress related to the above topics with trapped cold gases will be discussed. 


\section{Contents}

4

1.1. Cold atoms from a historical perspective 4

1.2. Cold atoms and the challenges of condensed matter physics 6

1.3. Plan of the review 10

2. The Hubbard and spin models with ultracold lattice gases 11

2.1. Optical potentials $\quad 12$

2.2. Hubbard models

2.3. Spin models $\quad 15$

2.4. Control of parameters in cold atom systems 17

2.5. Superfluid - Mott insulator quantum phase transition in the Bose Hubbard model 19

3. The Hubbard model: Methods of treatment 22

3.1. Introduction

3.3. Weak interactions limit 23

3.4. Strong interactions limit $\quad 25$

3.5. The Gutzwiller mean-field approach 26

3.6. Exact diagonalizations 28

3.7. Quantum Monte Carlo 29

3.8. Phase space methods 30

3.10. 1D methods 31

3.11. Bethe ansatz

3.12. A quantum information approach to strongly correlated systems

3.12.1. Vidal's algorithm 36

3.12.2. Matrix product states 38

3.13. Fermi and Fermi-Bose Hubbard models 39

4. Disordered ultracold atomic gases 43

4.1. Introduction

4.2. Disordered interacting bosonic lattice models in condensed matter 45

4.3. Realization of disorder in ultracold atomic gases 47

4.4. Disordered ultracold atomic Bose gases in optical lattices 49

4.5. Experiments with weakly interacting trapped gases and Anderson localization $\quad 52$

4.6 Disordered interacting fermionic systems

4.7 Disordered Bose-Fermi mixtures

4.8 Spin glasses 60

5. Frustrated models in cold atom systems 63

5.1. Introduction 63

5.2. Quantum antiferromagnets 64

5.2.1. The Heisenberg model 65

5.2.2. The $J_{1}-J_{2}$ model 66

5.3. Heisenberg antiferromagnets and atomic Fermi-Fermi mixtures in kagomé lattices 68 5.3.1. Heisenberg kagomé antiferromagnets

$\begin{array}{ll}\text { 5.3.2. Realization of kagomé lattice by Fermi-Fermi mixtures } & 69\end{array}$

5.4. Interacting Fermi gas in a kagomé lattice: Quantum spin-liquid crystals 69

5.4.1. The quantum magnet Hamiltonian 69

$\begin{array}{ll}\text { 5.4.2. Classical analysis } & 70\end{array}$

5.4.3. Quantum mechanical results 71

5.5. Realization of frustrated models in cold atom/ion systems 73

5.5.1. Simulators of spin systems with topological order 73

5.5.2. Frustrated models with polar molecules 74

$\begin{array}{ll}\text { 5.5.3. Ion-based quantum simulators of spin systems } & 75\end{array}$

6. Ultracold spinor atomic gases $\quad 77$

6.1. Introduction $\quad 77$

6.2. Spinor interactions $\quad 78$

6.3. $F=1$ and $F=2$ spinor gases: Mean field regime $\quad 79$

6.3.1. $\mathrm{F}=1$ gases in optical lattices 81

$\begin{array}{lr}\text { 6.3.2. Bose-Hubbard model for spin } 1 \text { particles } & 82\end{array}$

6.3.3. $\mathrm{F}=2$ gases in optical lattices 84

6.3.4. Bose-Hubbard model for $\mathrm{F}=2$ particles $\quad 85$

$\begin{array}{ll}\text { 6.3.5. Spinor Fermi gases in optical gases } & 88\end{array}$

7. Ultracold atomic gases in "artificial" magnetic fields 89 
7.1. Introduction - Rapidly rotating ultracold gases $\quad 89$

7.2. Lattice gases in "artificial" Abelian magnetic fields 91

7.3. Ultracold gases and lattice gauge theories 98

$\begin{array}{lr}\text { 8. Quantum information with ultracold gases } & 100\end{array}$

8.1. Introduction 100

8.2. Entanglement: A formal definition and some preliminaries 100

8.2.1. The partial transposition criterion for detecting entanglement 101

8.2.2. Entanglement measures 102

8.3. Entanglement and phase transitions $\quad 104$

8.3.1. Scaling of entanglement in the reduced density matrix 105

8.3.2. Entanglement entropy: Scaling of spin block entanglement 106

$\begin{array}{ll}\text { 8.3.3. Localizable entanglement and its scaling } & 107\end{array}$

8.3.4. Critical behaviour in the evolved state 108

8.4 Quantum computing with lattice gases 109

8.5. Generation of entanglement: The one-way quantum computer 111

8.5.1. The one-way quantum computer 111

8.5.2. Disordered lattice 113

9. Summary 114

Acknowledgements $\quad 114$

Appendix A: Effective Hamiltonian to second order $\quad 115$

Appendix B: Size of the occupation-reduced Hilbert space 117

References $\quad 117$ 
Motto:

There are more things in heaven and earth, Horatio,

Than are dreamt of in your philosophy [1].

\section{Introduction}

\subsection{Cold atoms from a historical perspective}

Thirty years ago, atomic physics was a very well established and respectful, but evidently not a "hot" area of physics. On the theory side, even though one had to deal with complex problems of many electron systems, most of the methods and techniques were developed. The main questions concerned, how to optimize these methods, how to calculate more efficiently, etc. These questions were reflecting an evolutionary progress, rather than a revolutionary search for totally new phenomena. Quantum optics at this time was entering its Golden Age, but in the first place on the experimental side. Development of laser physics and nonlinear optics led in 1981 to the Nobel prize for A.L. Schawlow and N. Bloembergen "for their contribution to the development of laser spectroscopy". Studies of quantum systems at the single particle level culminated in 1989 with the Nobel prize for H.G. Dehmelt and W. Paul "for the development of the ion trap technique", shared with N.F. Ramsey "for the invention of the separated oscillatory fields method and its use in the hydrogen maser and other atomic clocks".

Theoretical quantum optics was born in the 60ties with the works on quantum coherence theory by the 2005 Noble prize winner, R.J. Glauber $[2,3]$, and with the development of the laser theory by M. Scully and W.E. Lamb (Nobel laureate of 1955) [4], and H. Haken [5]. In the 70ties and 80ties, however, theoretical quantum optics was not considered to be a separate, established area of theoretical physics. One of the reasons of this state of art, was that indeed the quantum optics of that time was primarily dealing with single particle problems. Most of the many body problems of quantum optics, such as laser theory, or more generally optical instabilities [6], could have been solved either using linear models, or employing relatively simple versions of mean field approach. Perhaps the most sophisticated theoretical contributions concerned understanding of quantum fluctuations and quantum noise $[6,7]$.

This situation has drastically changed in the last ten - fifteen years, and there are several seminal discoveries that have triggered these changes:

- First of all, atomic physics and quantum optics have developed over the years quite generally an unprecedented level of quantum engineering, i.e. preparation, manipulation, control and detection of quantum systems.

- Cooling and trapping methods of atoms, ions and molecules have reached regimes of low temperatures (today down to nanoKelvin!) and precision, that 15 years ago were considered unbelievable. These developments have been recognised by the Nobel Foundation in 1997, who awarded the Prize to S. Chu [8], C. Cohen-Tannoudji [9] and W.D. Phillips [10] "for the development of methods to cool and trap atoms with laser light". Laser cooling and mechanical manipulations of particles with 
light [11] was essential for development of completely new areas of atomic physics and quantum optics, such as atom optics [12], and for reaching new territories of precision metrology and quantum engineering.

- Laser cooling combined with evaporation cooling technique allowed in 1995 for experimental observation of Bose-Einstein condensation (BEC) [13,14], a phenomenon predicted by S. Bose and A. Einstein more than 70 years earlier. The authors of these experiments, E.A. Cornell and C.E. Wieman [15] and W. Ketterle [16] received the Noble Prize in 2001, "for the achievement of Bose-Einstein condensation in dilute gases of alkali atoms, and for early fundamental studies of the properties of the condensates". This was a breakthrough moment, in which "atomic physics and quantum optics has met condensed matter physics" [16]. Condensed matter community at this time remained, however, still reserved. After all, BEC was observed in weakly interacting dilute gases, where it is very well described by the mean field Bogoliubov-de Gennes theory [17]. Although the finite size of the systems, and spatial inhomogeneity play there a crucial role, the basic theory of such systems was developed in the 50'ties.

- The seminal theoretical works of the late A. Peres [18], the proposals of quantum cryptography by C.H. Bennett and G. Brassard [19], and A.K. Ekert [20], the quantum communication proposals by C.H. Bennett and S.J. Wiesner [21] and C.H. Bennett, G. Brassard, C. Crépeau, R. Jozsa, A. Peres, and W.K. Wootters [22], the discovery of the quantum factorizing algorithm by P. Shor [23], and the quantum computer proposal by J.I. Cirac and P. Zoller [24] have given birth to experimental studies of quantum information $[25,26]$. These studies, together with rapid development of the theory have, in particular in the area of atomic physics and quantum optics, led to enormous progress in our understanding of what quantum correlations and quantum entanglement are, and how to prepare and use entangled states as a resource. The impulses from quantum information enter nowadays constantly into the physics of cold atoms, molecules and ions, and stimulate new approaches. It is very probable that the first quantum computers will be, as suggested already by Feynman [27], computers of special purpose - quantum simulators [28], that will efficiently simulate quantum many body systems that otherwise cannot be simulated using "classical" computers [26].

- The physics of ultracold atoms entered the areas of strongly correlated systems with the seminal 1998 paper of Jaksch et al. [29] on the superfluid-Mott insulator transition in cold atoms in an optical lattice. Apart from stimulation from the condensed matter physics [30], the authors of this paper were in fact motivated by the possibility of realising quantum computing with cold atoms in a lattice [31]. Transition to the Mott insulator state was supposed to be an efficient way of preparation of a quantum register with a fixed number of atoms per lattice site. The experimental observation of the superfluid-Mott insulator transition by the Bloch-Hänsch group [32] (see Fig. 1) marks the beginning of age of the experimental studies of strongly correlated systems with ultracold atoms [33]. Several other groups have observed bosonic superfluid-Mott insulator transitions in pure Bose systems [34], in disordered Bose systems [35], or in Bose-Fermi mixtures [36,37]. Very recently MI state of molecules have been created [38], and bound repulsive pairs of atoms (i.e. pairs of atoms at a site that cannot release their repulsive energy due to the band structure of the spectrum in the lattice) have been observed [39].

Since 1998 the physics of ultracold atoms has made enormous progress in the stud- 

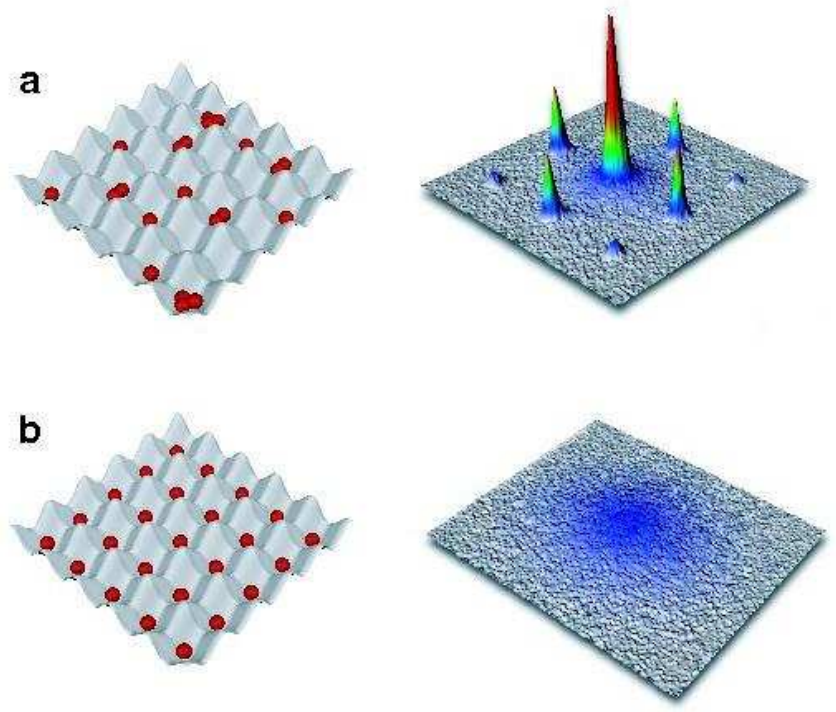

Figure 1. Distribution of atoms among lattice sites vs. momentum distribution of atoms released from an optical lattice. (a): low lattice potential: the largest interference peak is at zero momentum. The system is a superfluid with fluctuating number of atoms per site. (b): high lattice potential case where a perfect Mott state with exactly one atom per site is achieved - the momentum distribution is blurred (from [33]).

ies of strongly correlated systems. Number of the theory papers that propose to mimic various condensed matter systems of interest is hardly possible to follow, and the number of experiments in which strongly correlated regime has been met grows also very significantly. Moreover, condensed matter physicists, in particular theorists, joint the efforts of atomic physicists and quantum opticians. Among those who have "committed" a paper on cold atoms are Noble Prize winners: A.J. Leggett [40], F. Wilczek [41], D. Politzer [42], or such personalities of condensed matter theory as M.P.A. Fisher [43], or mathematical physics as M. Aizenman or E.H. Lieb [44].

\subsection{Cold atoms and the challenges of condensed matter physics}

The physics of cold atoms touches nowadays the same frontiers of modern physics as condensed matter and high energy physics. In particular many of the important challenges of the latter two disciplines can be addressed in the context of cold atoms:

- $1 D$ systems. The role of quantum fluctuations and correlations is particularly important in 1D. The theory of 1D systems is very well developed due to existence of exact methods such as Bethe ansatz and quantum inverse scattering theory (c.f. [45]), powerful approximate approaches, such as bosonisation, or conformal field theory [46], and efficient computational methods, such as density matrix renormalisation group technique (DMRG. c.f. [47]). There are, however, many open experimental challenges that have not been so far directly and clearly realized in condensed matter, and can be addressed with cold atoms (for a review see [48]). 
Examples include atomic Fermi, or Bose analogues of spin-charge separation, or more generally observations of microscopic properties of Luttinger liquids [49-51]. Recent experimental observations of the 1D gas in the deep Tonks-Girardeau regime by Paredes et al. [52] (see also [53-56]) are the first steps in this direction.

- Spin-boson model. A two state system coupled to a bosonic reservoir is a paradigm, both in quantum optics, as well as in condensed matter physics, where it is termed as spin-boson model (for a review see [57]). It has also been proposed [58] that an atomic quantum dot, i.e., a single atom in a tight optical trap coupled to a superfluid reservoir via laser transitions, may realise this model. In particular, atomic quantum dots embedded in a 1D Luttinger liquid of cold bosonic atoms realizes a spin-boson model with Ohmic coupling, which exhibits a dissipative phase transition and allows to directly measure atomic Luttinger parameters.

- 2D systems. According to the Mermin-Wagner-Hohenberg theorem, 2D systems with continuous symmetry do not exhibit long range order at finite temperatures T. 2D systems may, however, undergo Kosterlitz-Thouless-Berezinskii transition (KTB) to a state in which correlations decay is algebraic, rather than exponential. Although KTB transition has been observed in liquid Helium, its microscopic nature (binding of vortex pairs) has never been seen. A recent experiment of Dalibard's group [59] makes an important step in this direction.

- Hubbard and spin models. Very many important examples of strongly correlated state in condensed matter physics are realised in various types of Hubbard models $[45,60]$. While in condensed matter Hubbard models are "reasonable caricatures" of real systems, ultracold atomic gases in optical lattices allow to achieve practically perfect realizations of a whole variety of Hubbard models [61]. Similarly, in certain limits Hubbard models reduce to various spin models; again cold atoms and ions allow for practically perfect realizations of such spin models (see for instance [62-65]). Moreover, one can use such realizations as quantum simulators to mimic specific condensed matter models, and to address various very particular and well focused questions.

- Disordered systems: Interplay localisation-interactions. Disorder plays a central role in condensed matter physics, and its presence leads to various novel types of effects and phenomena. One of most prominent quantum signatures of disorder is Anderson localisation [66] of the wave function of single particles in a random potential. The question of the interplay between disorder and interactions has been intensively studied. For attractive interactions, disorder might destroy the possibility of superfluid transition ("dirty" superconductors). Weak repulsive interactions play a delocalising role, whereas very strong ones lead to Mott type localisation [67], and insulating behaviour. In the intermediate situations there exist a possibility of delocalized "metallic" phases. Cold atom physics starts to investigate these questions. Controlled disorder, or pseudo-disorder might be created in atomic traps, or optical lattices by adding an optical potential created by speckle radiation, or several lattices with incommensurate periods of spatial oscillations [68-70]. In an optical lattice this should allow to study Anderson-Bose glass and crossover between Anderson-like (Anderson glass) to Mott type (Bose glass) localisation. Very recently, Bose glass state has been realized experimentally by the M. Inguscio group [35]. The same group, as well as several others have initiated experimental and theoretical studies of the role of interactions in Anderson localisation effects for trapped Bose gases [71-74]. According to theoretical predictions of Ref. [73, 75-79], 
prospects of detecting signatures of Anderson localisation in the presence of weak nonlinear interactions and quasi-disorder in BEC are quite promising. One expects in such system the appearance of a novel Lifshits glass phase [80], where bosons condense in a finite number of states from the low energy tail (Lifshits tail) of the single particle spectrum.

- Disordered systems: spin glasses. Since the seminal papers of Edwards and Anderson [81], and Sherrington and Kirkpatrick [82] the question about the nature of the spin glass ordering has attracted a lot of attention [83-85]. The two competing pictures: the replica symmetry breaking picture of G. Parisi, and a droplet model of D.S. Fisher and D.A. Huse are probably applicable in some situations, and not applicable in others. Cold atom physics might contribute to resolving this controversy, and even add understanding of some quantum aspects, like for instance behaviour of Ising spin glasses in transverse (i.e. quantum mechanically non-commuting) fields [86,87].

- Disordered systems: Large effects by small disorder. There are many examples of such situations. In classical statistical physics a paradigm is the random field Ising model in 2D (that looses spontaneous magnetization at arbitrarily small disorder). In quantum physics the paradigmatic example is Anderson localisation, which occurs at arbitrarily small disorder in 1D, and should occur also at arbitrarily small disorder in 2D. Cold atom physics may address these questions, and, in fact, much more (cf. the Ref. [88], where a disorder breaks the continuous symmetry in a spin systems, and thus allows for long range ordering).

- High $T_{c}$ superconductivity. Despite many years of research, opinions on the nature of high $T_{c}$ superconductivity still vary quite appreciably [89]. It is, however, quite established (c.f. contribution of P.W. Anderson in Ref. [89]) that understanding of the 2D Hubbard model in the, so called, $t-J$ limit $[60]$ for two component $(\operatorname{spin} 1 / 2)$ fermions provides at least a part of the explanation. The simulation of these models are very hard and numerical results are also full of contradictions. Cold fermionic atoms with spin (or pseudospin) $1 / 2$ in optical lattices might provide a quantum simulator to resolve these problems [90] (see also [91]). First experiments with both "spinless", i.e. polarized, as well as spin 1/2 unpolarized ultracold fermions [34,92], and Fermi-Bose mixtures in lattices has already been realized [36, 37].

- BCS-BEC cross-over. Physics of high $T_{c}$ superconductivity can be also addressed with trapped ultracold gases. Weakly attracting spin $1 / 2$ fermions in such situations undergo at (very) low temperatures the Bardeen-Cooper-Schrieffer (BCS) transition to a superfluid state of loosely bounded Cooper pairs. Weakly repulsing fermions, on the other hand may form bosonic molecules, which in turn may form at very low temperatures a BEC. Strongly interacting fermions undergo also a transition to the superfluid state, but at much higher T. Several groups have employed the technique of Feshbach resonances [93-95] to observe such BCS-BEC cross-over (for the recent status of experiments see [96-106]).

- Frustrated antiferromagnets and spin liquids. The "rule of thumb" says that everywhere, in a vicinity of a high $T_{c}$ superconducting phase, there exists a (frustrated) antiferromagnetic phase. Frustrated antiferromagnets have been thus in the centre of interest in condensed matter physics for decades. Particularly challenging here is the possibility of creating novel, exotic quantum phases, such as valence bond solids, resonating valence bond states, and various kinds of quantum spin liquids (spin liquids of I and II kind, according to C. Lhuillier [107, 108], and topological 
and critical spin liquids, according to M.P.A. Fisher [109]). Cold atoms offer also in this respect opportunities to create various frustrated spin models in triangular, or even kagomé lattice [63]. In the latter case, it has been proposed by Damski et al. $[110,111]$ that cold dipolar Fermi gases, or Fermi-Bose mixtures might allow to realize a novel state of quantum matter: quantum spin liquid crystal, characterized by Néel like order at low $T$ (see also [112]), accompanied by extravagantly high, liquid-like density of low energy excited states.

- Topological order and quantum computation. Several very "exotic" spin systems with topological order has been proposed recently $[113,114]$ as candidates for robust quantum computing. Despite their unusual form, these models can be realized with cold atoms $[64,115]$. Particularly interesting [116] is the recent proposal by Micheli et al. [115], who propose to use heteronuclear polar molecules in a lattice, excite them using microwaves to the lowest rotational level, and employ strong dipoledipole interactions in the resulting spin model. The method provides an universal "toolbox" for spin models with designable range and spatial anisotropy of couplings.

- Systems with higher spins. Lattice Hubbard models, or spin systems with higher spins are also related to many open challenges; perhaps the most famous being the Haldane conjecture concerning existence of a gap, or its lack for the 1D antiferromagnetic spin chains with integer or half-integer spins, respectively (see for instance [60]). Ultracold spinor gases [117] might help to study these questions. Again, particularly interesting are in this context spinor gases in optical lattices [118-122], where in the strongly interacting limit the Hamiltonian reduces to a generalized Heisenberg Hamiltonian. Using Feshbach resonances and varying lattice geometry one should be able in such systems to generate variety of regimes and quantum phases, including the most interesting antiferromagnetic (AF) regime. GarcíaRipoll et al. [62] propose to use a duality between the AF and ferromagnetic (F) Hamiltonians, $H_{A F}=-H_{F}$, which implies that minimal energy states of $H_{A F}$ are maximal energy states of $H_{F}$, and vice versa. Since dissipation and decoherence are practically negligible in such systems, and affect equally both ends of the spectrum, one can study AF physics with $H_{F}$, preparing adiabatically AF states of interest.

- Fractional quantum Hall states. Since the famous work of Laughlin [123], there has been enormous progress in our understanding of the fractional quantum Hall effect (FQHE) [124]. Nevertheless, many challenges remain open: direct observation of the anyonic character of excitations, observation of other kinds of strongly correlated states, etc. FQHE states might be studied with trapped ultracold rotating gases $[125,126]$. Rotation induces there effects equivalent to an "artificial" constant magnetic field directed along the rotation axis. There are proposals how to detect directly fractional excitations in such systems [127]. Optical lattices might help in this task in two aspects: first, FQHE states of small systems of atoms could be observed in a lattice with rotating site potentials, or an array of rotating microtraps (cf. [128, 129] and references therein). Second, "artificial" magnetic field might be directly created in an lattices via appropriate control of tunneling (hopping) matrix element in the corresponding Hubbard model [130]. Such systems will also allow to create FQHE type states [131-133].

- Lattice gauge fields. Gauge theories, and in particular lattice gauge theories (LGT) [134] are fundamental for both high energy physics and condensed matter physics, and despite the progress of our understanding of LGT, many questions in this area remain open. Physics of cold atoms might help here in two aspects: "artificial" 
non-abelian magnetic fields may be created in lattice gases via appropriate control of hopping matrix elements [135], or in trapped gases using effects of electromagnetically induced transparency [136]. One of the most challenging tasks in this context concerns the possibility of realizing generalizations of Laughlin states with possibly non-abelian fractional excitations. Another challenge concerns the possibility of "mimicking" the dynamics of gauge fields. In fact, dynamical realizations of $U(1)$ abelian gauge theory, that involves ring exchange interaction in a square lattice [43], or 3 particle interactions in a triangular lattice $[137,138]$ have been also recently proposed.

- Superchemistry. This is a challenge of quantum chemistry, rather than condensed matter physics: to perform a chemical reaction in a controlled way, by using photoassociation or Feshbach resonances from a desired initial state to a desired final quantum state. Ref. [139] proposed to use MI with two identical atoms, to create via photoassociation, first a MI of homonuclear molecules, and then a molecular SF via "quantum melting". In Ref. [140], a similar idea was applied to heteronuclear molecules, in order to achieve molecular SF. Bloch's group have indeed observed photoassociation of ${ }^{87} \mathrm{Rb}$ molecules in $\mathrm{MI}$ with two atoms per site [141], while Rempe's group have realized the first molecular MI using Feshbach resonances [38]. Formation of three-body Efimov trimer states was observed in trapped Cs atoms by Grimm's group [142]. This process could be even more efficient in optical lattices [143].

- Ultracold dipolar gases. Some of the most facinating experimental and theoretical challenges of the modern atomic and molecular physics concern ultracold dipolar quantum gases (for a review, see [144]). The recent experimental realisation of the dipolar Bose gas of Chromium [145], and the progress in trapping and cooling of dipolar molecules [146] have opened the path towards ultracold quantum gases with dominant dipole interactions. Dipolar BECs and BCS states of trapped gases are expected to exhibit very interesting dependence on the trap geometry [144]. Dipolar ultracold gases in optical lattices, described by extended Hubbard models, should allow to realize various quantum insulating "solid" phases, such as checkerboard, and superfluid phases, such as supersolid phase [147, 148]. Particularly interesting in this context are the rotating dipolar gases (RDG). Bose-Einstein condensates of RDGs exhibit novel forms of vortex lattices: square, "stripe crystal", and "bubble crystal" lattices [149]. We have demonstrated that pseudo-hole gap survives the large $N$ limit for the Fermi RDGs [150], making them perfect candidates to achieve the stongly correlated regime, and to realise Laughlin liquid at filling $\nu=1 / 3$, and quantum Wigner crystal at $\nu \leq 1 / 7$ [151] with mesoscopic number of atoms $N \simeq 50-100$.

Several of the above mentioned open questions and challenges are addressed in this review. However, before we turn to the discussion of how ultracold atomic gases can mimic condensed matter systems, let us discuss shortly the properties of optical potentials in general, and optical lattices in particular (for a review, see [152]).

\subsection{Plan of the review}

This review is addressed to two kinds of readers. First of all, it gives for condensed matter and, perhaps, high energy physicists an overview of what is being done in 
atomic physics and quantum optics in the area of ultracold gases in optical lattices. The particular emphasis is put here on the problems that are directly related to open problems and challenges of condensed matter, or even high energy physics. We discuss how to mimic condensed matter, and even go beyond toward completely new areas and problems. Second, the review is directed to atomic and quantum optics community. For these readers it should give some basic information and basic literature about challenging problems of condensed matter physics that can be attacked with atoms, ions or molecules.

The plan of the review is as follows. In Section 2, we review the most general type of Hubbard-type model that can be realized with cold gases, and also review some spin models that can be reduced from the Hubbard model in specific limits. In Section 3, we present some basic theoretical methods of treatment of Hubbard models. Most of the material here is standard in condensed matter theory, but we include also a subsection about very recent developments in numerical treatments of many body systems based on quantum information and quantum optics ideas. In the following sections we address some of the challenges and open question described in this introduction. Each section has its own short introduction with basic condensed matter references to the considered problems, and then focuses on results obtained within the atomic physics and quantum optics context. Section 4 treats disordered ultracold gases, section 5 - frustrated ultracold gases, while sections 6 and 7 make short overviews of spinor ultracold gases and ultracold gases in "artificial" magnetic fields, respectively. The final section 8 discusses relations between ultracold gases and quantum information.

Since the review intends to strength the analogies between condensed matter systems and cold gases in optical lattices, it is mainly focused on the strongly interacting regime. Nevertheless, there is a wide range of interesting phenomena that appears in the weakly interacting regime that are not cover here.

This review has been written by theorists, and as such describes experiments only in aspects concerning the physical results, or the experimentally accessible ranges of parameters. We do not discuss here experimental techniques and methods. This review should be considered as complementary to the excellent review by Bloch and Greiner [153].

\section{The Hubbard and spin models}

In this section, we begin by a short discussion on optical potentials (Subsec. 2.1), and then review the most general Hubbard-type model that can be realized with cold gases (Subsec. 2.2). We report then (in Subsec. 2.3) the spin models that such Hubbard models reduce to, in different limits. In Subsec. 2.4, we discuss the amount of control that we have in the parameters involved in the Hubbard model, when realized with cold atoms. We then focus (in Subsec. 2.5) on the the paradigmatic model of a system that exhibits a quantum phase transition, namely on the homogeneous BoseHubbard $(\mathrm{BH})$ model $[30,154]$. The model undergoes the superfluid-Mott insulator (SF-MI) quantum phase transition. 

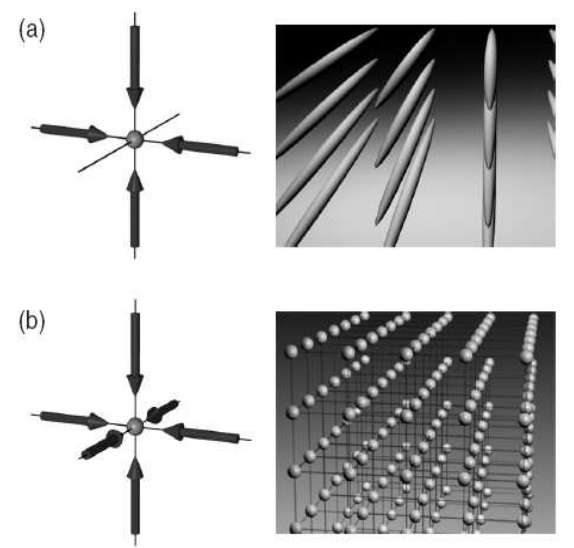

Figure 2. Schematic pictures of optical lattice potentials; a) 2D square lattice of quasi 1D traps; b) $3 \mathrm{D}$ simple cubic lattice (from [33]).

\subsection{Optical potentials}

The basic tool to create ultracold lattice gases are optical potentials. An electron in an atom in the presence of oscillating electric field $\mathbf{E}(\mathbf{r}, t)$ of a laser attains a time dependent dipole moment $\mathbf{d}$. When the field oscillations are far off resonance (i.e. they do not cause any real transition in the atom), the induced dipole moment follows the laser field oscillations,

$$
d_{i}=\sum_{j=x, y, z} \alpha_{i j}\left(\omega_{L}\right) E_{j}(\mathbf{r}, t),
$$

where $d_{i}$ is the corresponding component of $\mathbf{d}(i=x, y, z), \omega_{L}$ is the laser frequency, and $\alpha_{i j}\left(\omega_{L}\right)$ denotes the matrix elements of the polarizability tensor. The polarizability depends in general on the laser frequency, and on the energies of the non-resonant excited states of the atom. One of these states (with excitation energy, say, $E_{1}=\hbar \omega_{1}$ ) is usually much closer to the resonance than the others; in such case the polarizability becomes inversely proportional to the laser detuning from the resonance, $\Delta=\omega_{L}-\omega_{1}$.

Electronic energy undergoes in this situation a shift, $\Delta E$, which is nothing else than a AC-version of the standard quadratic Stark effect. The energy shift is proportional to

$$
\Delta E(\mathbf{r})=\sum_{i, j=x, y, z} \alpha_{i j}\left(\omega_{L}\right)\left\langle E_{j}(\mathbf{r}, t) E_{j}(\mathbf{r}, t)\right\rangle \propto I(\mathbf{r}) / \Delta,
$$

where the bra-ket denotes the averaging of the product of electric fields over the fast optical oscillations, and $I(\mathbf{r})$ is the laser beam intensity.

The consequences of the above simple formula are enormous. The atom feels an optical potential $V_{\text {opt }}(\mathbf{r})=\Delta E(\mathbf{r})$, that follows the spatial pattern of the laser field intensity. This is the basis for optical manipulations and trapping of atoms! If the laser is red-detuned (i.e., laser detuning $\Delta<0$ ), the atoms are attracted toward the 
regions of high intensity; conversely, blue detuned laser pushed the atoms out of the regions of high intensity.

Adding two, or more laser fields of the same frequency leads in general to interferences and the corresponding interference pattern of the intensity. In particular, two counter propagating laser waves of the same polarisation will create a standing wave, and thus a spatially oscillating potential for atoms. One can easily avoid interferences when adding more and more laser fields, and add corresponding intensities, i.e. optical potentials. To this aim one can use laser fields with orthogonal polarisations. Three pairs of counter-propagating laser beams with orthogonal polarizations will form then the 3D optical lattice represented schematically in Fig. 2b. An alternative way to avoid interferences on demand is to use slightly, but sufficiently different frequencies. In this case, time averaging over the "sufficient" different frequencies washes out the interference effects. Similarly, one can use laser beams with polarisations oscillating at different frequencies to avoid interferences.

\section{$2.2 \quad$ Hubbard models}

We are interested in the Hubbard-type models that are realizable with cold atoms in an optical lattice. The simplest optical lattice is a 3D simple cubic lattice, as presented in Fig. 2b. It is formed by three pairs of laser beams creating three orthogonal standing waves with orthogonal polarisations. As we will discuss below, one can, however, create practically arbitrary lattices on demand using optical potentials. Also, as the intensity of one of the standing waves increases, the probability of hopping along this direction decreases rapidly to zero [29]. In effect we obtain an 1D array of 2D square lattices. Consequently, an increase of the laser intensity of another of the standing waves, creates effectively a $2 \mathrm{D}$ array of $1 \mathrm{D}$ lattices (Fig. 2a).

Optical lattices provide an ideal (contain no defects) and rigid (do not support phonon excitations ${ }^{1}$ ) periodic potential in which the atoms move. As it is well known from solid state theory [155], single particle energy spectrum (in the absence of interactions) consists of bands, and the energy eigenstates of the Hamiltonian are Bloch functions. If the lattice potential is strong, the band-gaps are large, and the bands are very well separated energetically. For low temperatures regime, it is easy to achieve a situation in which only the lowest band is occupied (tight binding approximation). The Bloch functions of the lowest band can be expanded in Wannier functions, which are not the eigenstates of the single particle Hamiltonian, but are localised at each site. In the tight binding approximation, we project all of the atomic quantum field operators that describe the systems in question onto the lowest band, and then expand into the Wannier basis. Leaving then just the most relevant terms in the Hamiltonian (such as hopping between the nearest neighboring sites) leads directly to Hubbardtype Hamiltonian.

Let us write the most general Hubbard model that may be created in this way. Let us assume that we have several bosonic and fermionic species (or bosons/fermions with several internal states), enumerated by $\alpha$. The basic objects of the theory will be thus annihilation and creation operators of $\alpha$-bosons, $b_{i \alpha}, b_{i \alpha}^{\dagger}$ at the site $i$, and analogously annihilation and creation operators of $\beta$-fermions, $f_{i \beta}, f_{i \beta}^{\dagger}$ at the site $i$.

\footnotetext{
${ }^{1}$ This statement has to be revised when the lattice is created inside of an optical cavity. As we discuss later, the presence of atoms may affect the cavity field.
} 
Bosonic (fermionic) operators fulfill, of course, the standard canonical commutation (anticommutation) relations:

$$
\begin{aligned}
& {\left[b_{i \alpha}, b_{j \alpha^{\prime}}\right]=\left\{f_{i \alpha}, f_{j \alpha^{\prime}}\right\}=0} \\
& {\left[b_{i \alpha}, b_{j \alpha^{\prime}}^{\dagger}\right]=\left\{f_{i \alpha}, f_{j \alpha^{\prime}}^{\dagger}\right\}=\delta_{\alpha \alpha^{\prime}} \delta_{i j},}
\end{aligned}
$$

where $\delta_{a b}$ denotes the Kronecker delta.

The most general Hubbard type of hamiltonian that one can realize with cold atoms, assuming lowest band occupation only ${ }^{1}$, consists of four parts:

$$
H_{\text {Hubbard }}=H_{\text {hop }}+H_{\text {int }}+H_{\text {pot }}+H_{R a b i} .
$$

The hopping part describes hopping (tunneling) of atoms from one site to another. Since the hopping probability amplitude decreases exponentially with the distance, hopping is typically assumed to occur between the nearest neighboring sites, denoted $\langle i j\rangle$. Hopping, on the other hand, might be laser assisted, and thus might allow for transitions from one internal state to another (of course, or perhaps unfortunately, it cannot lead to a change of element, or even isotope!),

$$
H_{h o p}=-\sum_{\alpha, \beta,\langle i j\rangle}\left[t_{i j \alpha \beta}^{B} b_{i \alpha}^{\dagger} b_{j \beta}+\text { h.c. }\right]-\sum_{\alpha, \beta\langle i j\rangle}\left[t_{i j \alpha \beta}^{F} f_{i \alpha}^{\dagger} f_{j \beta}+\text { h.c. }\right] .
$$

Atoms interact in the first place via short range Van der Waals forces, which in the low energy limit are very well described by various kinds of the zero range pseudopotentials $[17,157,158]$. That means that the dominant part of the interactions is of contact type, i.e. occurs on-site. There are, however, situations in which interactions do affect neighbouring sites, or even have long range (such as dipole-dipole interactions). We thus write the interaction part as

$$
H_{\text {int }}=H_{\text {on-site }}+H_{\text {ext }},
$$

where

$$
\begin{aligned}
H_{\text {on-site }} & =\frac{1}{2} \sum_{i, \alpha, \beta, \alpha^{\prime} \beta^{\prime}}\left[U_{\alpha \beta \alpha^{\prime} \beta^{\prime}}^{B B}(i) b_{i \alpha}^{\dagger} b_{i \beta}^{\dagger} b_{i \beta^{\prime}} b_{i \alpha^{\prime}}+U_{\alpha \beta \alpha^{\prime} \beta^{\prime}}^{F F}(i) f_{i \alpha}^{\dagger} f_{i \beta}^{\dagger} f_{i \beta^{\prime}} f_{i \alpha^{\prime}}\right. \\
& \left.+2 U_{\alpha \beta \alpha^{\prime} \beta^{\prime}}^{B F}(i) b_{i \alpha}^{\dagger} f_{i \beta}^{\dagger} f_{i \beta^{\prime}} b_{i \alpha^{\prime}}\right] .
\end{aligned}
$$

In the simplest Hubbard models interactions depend only on the on-site atom numbers, $n_{i \alpha}^{B}=b_{i \alpha}^{\dagger} b_{i \alpha}$, and $n_{i \alpha}^{F}=f_{i \alpha}^{\dagger} f_{i \alpha}$. In general, however, they may depend in an non-trivial way on internal states, or atomic species; this is for instance the standard case for spinor gases [117]. The models with non-contact interactions are usually termed as extended Hubbard models, and include

$$
H_{e x t}=\frac{1}{2} \sum_{i, j \alpha, \beta, \alpha^{\prime}, \beta^{\prime}}\left[V_{\alpha \beta \alpha^{\prime} \beta^{\prime}}^{B B}(i, j) b_{i \alpha}^{\dagger} b_{j \beta}^{\dagger} b_{j \beta^{\prime}} b_{i \alpha^{\prime}}+V_{\alpha \beta \alpha^{\prime} \beta^{\prime}}^{F F}(i, j) f_{i \alpha}^{\dagger} f_{j \beta}^{\dagger} f_{j \beta^{\prime}} f_{i \alpha^{\prime}}\right.
$$

\footnotetext{
${ }^{1}$ Some authors go beyond this assumption. See for instance Ref. [156].
} 


$$
\left.+2 V_{\alpha \beta \alpha^{\prime} \beta^{\prime}}^{B F}(i, j) b_{i \alpha}^{\dagger} f_{j \beta}^{\dagger} f_{j \beta^{\prime}} b_{i \alpha^{\prime}}\right] .
$$

Most typically, the non-contact interactions will depend on the distance between the sites, $\left|\mathbf{r}_{i}-\mathbf{r}_{j}\right|$, and will be of the density-density form (i.e. they depend only on $n_{i \alpha}^{B}$, and $n_{i \beta}^{F}$ ), but in general, again this does not have to be the case. Dipolar interactions, for instance, depend on the angles between the dipole moments and on the vector $\mathbf{r}_{i}-\mathbf{r}_{j}$.

The last two parts of the Hamiltonian (Eq. (5)) describe on-site single atom processes, and essentially have the same form as the tunneling part, except that they occur on-site. We do distinguish them since they are well defined and controllable in experiments. $H_{\text {pot }}$ combines effects of all potentials felt by atoms such as external trapping potential, possible additional superlattice (i.e. additional lattice) potentials, possible disorder potentials, and last, but not least, chemical potential which is necessary if one uses the statistical description based on the grand canonical ensemble:

$$
H_{p o t}=-\sum_{\alpha, i}\left[\mu_{i \alpha}^{B} b_{i \alpha}^{\dagger} b_{i \alpha}+\mu_{i \alpha}^{F} f_{i \alpha}^{\dagger} f_{i \alpha}\right]
$$

The last part of the Hamiltonian, $H_{R a b i}$, describes possible coherent on-site transitions between the internals states of atoms; such transitions may be achieved using laser induced resonant Raman transitions, or microwave Rabi type transitions (we can write this part of the Hamiltonian as time-independent in the interaction picture with respect to the on site internal states Hamiltonian)

$$
H_{R a b i}=\frac{1}{2} \sum_{\alpha, \beta, i}\left[\Omega_{i \alpha \beta}^{B} b_{i \alpha}^{\dagger} b_{i \beta}+\Omega_{i \alpha \beta}^{F} f_{i \alpha}^{\dagger} f_{i \beta}\right] .
$$

\section{$2.3 \quad$ Spin models}

As it is well known (see for instance [60]), Hubbard models reduce to spin models in certain limits. Most of these limits, and even more, are accessible with cold atoms. Generally speaking, if bosonic atoms can occupy only $2 S+1$ different states in a lattice site, then one can always map these states onto the states of pseudo-spin $S$. These states may even correspond to different number of bosons, and the dimension of the local, on-site Hilbert space might vary in space; in such case we will deal with inhomogeneous models, where at each site there is, in general, a different spin. Similar construction might be done for fermionic atoms, with the remark that at a given site, the fermion number differences might attain only even numbers, since otherwise the fermionic character of particles cannot be eliminated.

When constructing specific spin models two aspects play a role: lattice geometry (which we discuss in the next subsection), and the form of interactions, which includes Ising, $X Y$, Heisenberg, $X X Z$, and anisotropic $X Y Z$ types, as well as ring exchange types. Below we list the most obvious constructions of spin models, that has been discussed in the literature on cold atoms. We restrict ourselves here to translationally invariant models.

- Hard core bosons and XY models. Perhaps the simplest way to obtain a non-trivial spin model is to use the simplest Bose-Hubbard Hamiltonian for one component 
("spinless") bosons

$$
H=-t \sum_{\langle i j\rangle}\left[b_{i}^{\dagger} b_{j}+h . c .\right]+\frac{U}{2} \sum_{i} n_{i}\left(n_{i}-1\right)-\mu \sum_{i} n_{i}
$$

where $\langle i j\rangle$ denotes sum over nearest neighbors. In the hard boson limit (i.e. when $U \gg t, \mu$ ) we may have at most 1 boson per site. We may encode the spin $1 / 2$ states as presence $(\uparrow)$, or absence $(\downarrow)$ of the boson at the site. The Hamiltonian reduces then to that of $X Y$ model in a transverse field,

$$
H=-t \sum_{\langle i j\rangle}\left[\sigma_{i}^{\dagger} \sigma_{j}+\text { h.c. }\right]-\frac{\mu}{2} \sum_{i}\left(\sigma_{z, i}+1\right),
$$

where $\sigma_{i}=\left(\sigma_{x, i}+i \sigma_{y, i}\right) / 2$ and $\sigma_{x, y, z, i}$ denote the standard Pauli matrices at site $i$. This model has the advantage that in $1 \mathrm{D}$ it is exactly solvable via Jordan-Wigner transformation [154]. One interesting application of this approach concerns the 1D disordered chain studied in Ref. [159]. The same approach was used recently in [88] to realize $X Y$ model in random parallel field.

- Spatially delocalised qubits. Somewhat similar idea considers two neighbouring traps, or potential wells ("left" and "right"), assumes 1 atom per double well, and encodes the spin $1 / 2$ (qubit) as the presence of the atom on the "left", or "right", respectively [160]. In Ref. [65] was proposed to encode one qubit by the presence or the absence of a whole string of neutral atoms yielding improved robustness. This system may be used for generation of maximally entangled many atom states (Schrödinger cat states) by crossing a quantum phase transition.

- Multi-component atoms in Mott states. Whenever we deal with a system of multicomponent atoms, i.e. atoms with say $2 S+1$ internal states, in the Mott insulator limit, the system will be well described by the appropriate spin model. The most prominent example is a two-component (or spin 1/2) Fermi gas [60], which in the Mott state with one atom per site forms a perfect Heisenberg model. Several groups are planning experiments with ultracold spin 1/2 Fermi atoms heading toward antiferromagnetism in various kinds of lattices. Prospects for observing antiferromagnetic transition in such systems are quite good, especially since one expect to be able to employ interaction induced cooling (an analogue of the Pomeranchuk effect, known in liquid Helium physics), [161], or disorder induced increase of $T_{c}$ [88]. One should stress, however, that although the Mott transition takes typically place when $t<U$, the low temperature physics of the resulting Heisenberg model requires temperatures of order $t^{2} / U \simeq k_{B} T$. Such temperatures are often in the nanoKelvin range, i.e. still hard to achieve experimentally. There is, however, a lot of new proposals of cooling atoms in the Mott states [162-165], and hopefully the temperatures will not set any limitations on experiments with these kinds of spin models in the next future.

The calculation of the effective Hamiltonians in pseudo-spin 1/2 Bose-Bose or BoseFermi mixtures is quite complicated, and was accomplished for the first time recently $[166,167]$. The Bose-Bose case can be reduced to an XXZ spin model (for the case of XXZ model in random fields see for instance [88]). The effective Hamiltonian for the Fermi-Bose mixture in general cannot be reduced to a spin model, since it involves Fermi operators describing composite fermions, consisting of one fermion 
paired with some number of bosons, or bosonic holes [167]. The Hamiltonian describes a "spinless" interacting Fermi gas of such composites. It can, however, be transformed to an XXZ model in external fields in $1 \mathrm{D}$ via Jordan-Wigner transformation.

- Spinor gases in Mott states. Of course, the above statements are particularly valid for spinor gases, which for atoms with spin $F>1 / 2$ have effective Hamiltonians containing generalizations (a power series) of Heisenberg interactions. For $F=1$ and the Mott state with 1 atom per site, we deal with the so-called QuadraticBiquadratic Hamiltonian [62,118-121]. In the Mott state with two atoms per site, the pair can either compose a singlet state or a state with on-site spin $S=2$. The resulting Hamiltonian contains then higher powers of the Heisenberg term. The situation is obviously more complicated for higher Mott states, and atoms with higher individual spin $F$. In Refs. [122, 168, 169], the case $F=2$ is studied; here, already with one atom per site, the effective Hamiltonian is a polynomial of the fourth order in Heisenberg term.

- Spin models in polymerized lattices. Yet another interesting way to obtain spin models with cold atoms, is based on the use of polymerized (dimerized, trimerized, quadrumerized etc.) lattices. These are lattices that can be easily realised with optical potentials, and have no analogue in condensed matter physics. A simple example of dimerized lattice in $2 \mathrm{D}$ is a square lattice of pairs of close sites; trimerized kagomé lattice, discussed in section 4, is a triangular lattices of trimes of close sites located on a small unilateral triangle; $2 \mathrm{D}$ quadrumerized square lattice is a square lattice of small squares of close sites, etc. When one considers ultracold gases in such lattices, one has to take into account first the lowest energy state in a dimer, trimer etc. If we deal with polarized "spinless" fermions in trimerized lattice, and we consider two fermions per trimer, fermions have to their disposal zero momentum state (which will be necessarily filled at low temperatures), and the two states with left and right chirality. The latter two are obviously degenerated, and can thus be used to encode the effective spin $1 / 2$. This is the model discussed in Refs. $[63,110,111]$. Note, that similar encoding is possible in a quatromerized lattice with 2 atoms per quadrumer.

\subsection{Control of parameters in cold atom systems}

Atomic physics and quantum optics offer many new types of methods to quantum engineer systems in question. Toutes proportions gardées, there are instances in which atomic physics and quantum optics not only meets, but rather "beats" condensed matter physics. That is one of the reasons why the physics of ultracold atoms attracts so many theorists from other disciplines.

Let us list shortly what can be controlled in the experiments with cold atoms in optical lattices:

- Lattice geometry and dimensionality. As mentioned above, practically any lattice geometry may be achieved with optical potentials. The method of superlattices (i.e. adding a new lattice on top of the exiting one) is very well developed. Changing of the lattice dimentionality does not pose any problem (compare Fig. 2). Also periodic boundary conditions can be realized in ring shapeed optical lattices [170].

- Phonons. Optical lattices are rigid and robust: they do not have any phonons. An 
interesting situation arises when the lattice is formed in an optical cavity: atomlight coupling might suffice then to shift the cavity resonance. Cavity will affect the atoms, but atoms will perform back action, and create "phonon" like excitations. For early works, see $[171,172]$. For more recent studies of superfluid-Mott insulator crossover, see [173], and for the first attempts towards "refracton" physics (analogs of "phonons"), see [174]. Phonons, obviously, play a role in ion traps, where they provide the major mechanism for ion-ion interactions.

- Tunneling. Tunneling can be controlled to a great extend using combination of pure tunneling, laser assisted coherent transitions, and lattice tilting (acceleration) techniques. The prominent example of such control describe the proposals for creating artificial magnetic fields [130-133,135].

- On-site interactions. These interactions are controlled by scattering lengths, which can be modified using Feshbach resonances in magnetic fields, [93-95], or optical Feshbach resonances (for theory see $[175]$, for experiments $[176,177]$ ). On-site interactions can be set to zero in dipolar gases, by changing the shape of the on-site potential [147].

- Next neighbour and long range interactions. Effective models obtained by calculating effect of tunneling in the Mott insulator phases, contain typically short range interactions of energies $\propto t^{2} / U$. Stronger interactions can be achieved using dipolar interactions, such as those proposed in Refs. $[115,147,178]$. Dipolar interactions are of long range type, are anisotropic, and exhibit a very rich variety of phenomena (for a review, see [144]). They can also be achieved in trapped ion systems, where they are mediated via phonon vibrations of the equilibrium ionic configuration. This case is discussed in detail in the section 7 of this review. The group of T. Pfau has recently realized the first experimental observation of ultracold dipolar gas [145], by condensing bosonic Chromium. Dipolar interaction are mediated here by magnetic dipoles of the Chromium atoms; they are weak, but nevertheless lead to observable effects [179].

- Multiparticle (plaquette) interactions. It has been also demonstrated how to generate effective three-body interactions in triangular optical lattices [137]. These interactions result from the possibility of atoms tunneling along two different paths. Similarly, ring exchange interactions in square optical lattice can be generated employing the correlated hopping of two bosons [43].

- Potentials. Various types of external potentials can be applied to the atoms, depending on the situations. One can use magnetic potentials whose shape can be at least cotrolled on the scale of few microns. Magnetic potentials with larger gradients can be created on atom chips (cf. [180]). The most flexible are, however, optical potentials. Apart from limitations set by the diffraction limit, they can have practically any desired shape and can form any kind of optical lattice: regular, disordered, modulated, etc. Recently, J. Schmiedmayer demonstrated also great possibilities offered by the so called radio frequency potentials [181].

- Rabi transitions. Similarly, apart from limitations set by diffraction, they are in practice highly controlable.

- Temperatures. The typical critical temperatures $T$ of trapped ultracold condensed Bose gases are of order of nano-Kelvins. Using evaporative cooling one can reach, however, lower $T$ (which in fact are not very well known, because of the lack of reliable temperature measurement methods. For recent advances see [182]). Sim- 
ilarly, the temperature of superfluid Fermi gases are in the range of tens of nK. One can thus say that temperatures in the range of tens of $\mathrm{nK}$ are becoming nowadays a standard. There are many proposals for reaching even lower $T$ s employing additional cooling and filtering procedures [165]. SF-MI transition occurs in the regime of $T$ s accessible nowadays. Many of the strongly correlated phases occur in the regime when the tunneling $t$ is much smaller than $U$ and require temperatures of order $k_{B} T \simeq t^{2} / U$, i.e. $10-20 \mathrm{nK}$, or even less. This is at the border of the current possibilities, but the progress in cooling and quantum engineering techniques allow us to believe that these limitations will be overcome very soon (for a detailed discussion see [183])

- Time dependences. The time scales of coherent unitary dynamics of these systems are typically in the millisecond range. It implies that, in contrast to condensed matter systems, all of the controls discussed above can be made time dependent, adiabatic, or diabatic, on demand. Some of the fascinating possibilities include change of lattice geometry, or turn-on of the disorder in real time.

The huge range of parameters which are experimentally controllable indicates the rich possibilities offered by cold gases in optical lattices to implement condensed matter models and beyond. The first proof came with the seminal paper of Greiner et al. [32] reporting the superfluid-Mott insulator transition with cold bosons in an optical lattice.

\subsection{Superfluid - Mott insulator quantum phase transition in the Bose Hubbard model}

Let us now consider the ideal homogeneous Bose-Hubbard ( $\mathrm{BH})$ model of the form

$$
H=-t \sum_{\langle i, j\rangle}\left(b_{i}^{\dagger} b_{j}+h . c .\right)+\frac{U}{2} \sum_{i} n_{i}\left(n_{i}-1\right),
$$

where $\langle i, j\rangle$ indicates sum over nearest neighbors. We denote here the tunneling energy by $t$ (both $t$ and $J$ are used in the literature). Below we discuss the superfluid (SF) Mott insulator (MI) quantum phase transition separately for the case of transitions at fixed density, and for the, so-called, generic (density-driven) transitions, where the number of atoms changes:

- Transitions at fixed integer density. We consider here transitions driven by a change of $t / U$ ratio in a system with a fixed number of bosons. The phase transition occurs when the lattice filling factor $\bar{n}$ (the number of atoms per site) is exactly integer. For $t / U<(t / U)_{c} \ll 1$ there is a Mott insulator (MI) phase, while for $t / U>$ $(t / U)_{c}$ there is a superfluid (SF) phase. As discussed by Fisher et al. [30], such a transition in a $d$-dimensional $\mathrm{BH}$ model lies in the universality class of the $(d+1)$ dimensional $X Y$ spin model. This result implies that the one dimensional BoseHubbard model undergoes a Kosterlitz-Thouless phase transition. Also it permits to determine the critical exponents. The quantum phase transition happens, ideally, at absolute temperature equal to zero [154], and its signatures are reflected in different quantities as discussed below.

The superfluid fraction $[184,185]$, which is defined through the response of the system to an externally imposed velocity field (equivalent to a twist in boundary 
conditions) vanishes in the Mott phase. This was verified in a 1D system numerically by a DMRG calculation [186], and analytically in a simplified BH model subjected to the restriction of a maximal site occupation of two particles [187]. Additionally, a jump in the superfluid fraction was observed at the transition point [186] of a one dimensional system, a result expected at the Kosterlitz-Thouless critical point [188]. It is worth to stress here, that the superfluid fraction should not be confused with the condensate fraction [40]. The latter one is equal to the highest eigenvalue of the single particle density matrix $\left\langle b_{i}^{\dagger} b_{j}\right\rangle$, divided by the number of particles. These quantities, both equal 100\% for an untrapped 3D Bose-Einstein condensate in the dilute limit [185], can be very different in the Bose-Hubbard model, see e.g. [68] for a simple example where the condensate fraction is $100 \%$, while the superfluid fraction hits zero once a sufficiently strong on-site disorder is present.

The excitation spectrum is gapless for the SF phase while gapped for the MI. In the MI neighborhood of the transition point, the gap scales exponentially in a one dimensional system as $\propto \exp \left(\sim\left[(t / U)_{c}-t / U\right]^{-1 / 2}\right)$, where the proportionality factor in the exponent is smaller than zero. In two and three dimensional models, it exhibits a power law behaviour $\propto\left[(t / U)_{c}-t / U\right]^{z \nu}$, where $z$ and $\nu$ are the critical exponents $[30,154]$. These exponents for a two dimensional system are $z=1$ and $\nu \approx 0.67$ [189], and for a three dimensional model they read as $z=1$ and $\nu=1 / 2[30]$.

Another quantity that shows a critical behaviour is the correlation length $\xi$ :

$$
\xi^{2}=1 / 2 \sum_{r} r^{2}\left\langle b_{j}^{\dagger} b_{j+r}+h . c .\right\rangle / \sum_{r}\left\langle b_{j}^{\dagger} b_{j+r}+h . c .\right\rangle .
$$

It is finite in the Mott phase, diverges at the critical point and stays divergent in the superfluid phase. In the neighborhood of the critical point it behaves as: $\xi \sim$ gap $^{-1 / z}$, where the critical exponent $z=1$ in one, two, and three dimensional systems [30].

Additional insight into the superfluid-Mott insulator phase transition can be obtained investigating the relation between $\mu / U, t / U$, and the number of particles in the system. The chemical potential $\mu$ is conveniently introduced during minimization of $\left\langle H-\mu \sum_{i} n_{i}\right\rangle$ leading to the determination of ground state with a $\mu$-dependent total number of atoms. When $t / U=0$, one obtains that for $\bar{n}-1<\mu / U<\bar{n}(\bar{n}$ is an integer number) the ground state is a single Fock state

$$
|\bar{n}, \bar{n}, \ldots\rangle,
$$

When tunneling is nonzero, the range of $\mu / U$ describing the system with integer $\bar{n}$ gradually shrinks and finally disappears at some $\left\{(\mu / U)_{c},(t / U)_{c}\right\}$. In this way, the famous lobes are formed: see Fig. 3. The fixed density transition happens when the system moves along the thin solid lines depicted in Fig. 3. This schematic plot illustrates also that a system with a non-integer filling factor never enters the MI lobes, i.e., stays always superfluid as depicted by the dashed lines. It is easily understood by considering a state with one particle added (subtracted) to (from) a system having integer filling factor. Such a particle (hole) can freely flow through the lattice, so that the system becomes superfluid for all values of $t / U$ ratio.

It is important to stress that though inside the lobes the filling factor $\bar{n}$ is integer, the ground state is not a single Fock state $(16)$ for $t / U \neq 0$. To illustrate this fact 


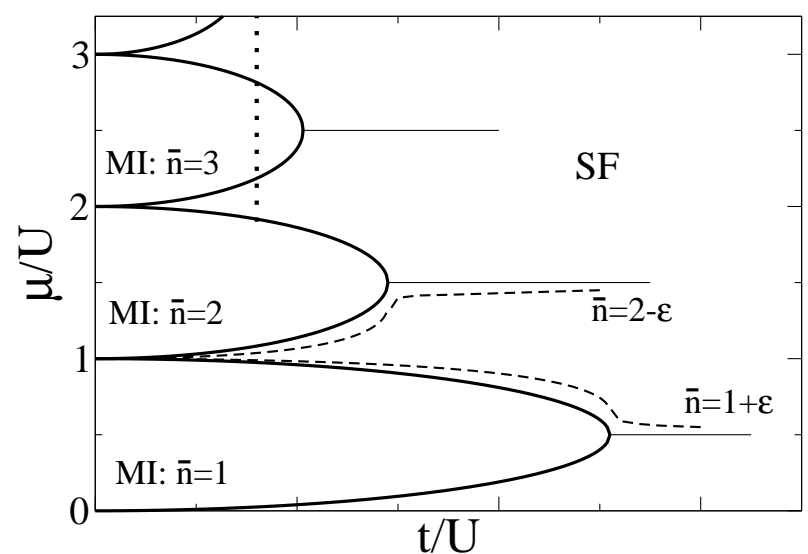

Figure 3. A schematic plot of the phase diagram of the Bose-Hubbard model. The lobes, surrounded by the superfluid sea, correspond to the Mott insulator islands with integer filling factor $\bar{n}$. The thin solid lines represent the lines of constant, integer density. The dashed lines show trajectories of a system with fixed, non-integer, filling factor $\bar{n}(0<\varepsilon \ll 1)$. The dotted line presents an example of a trajectory leading to a generic phase transition when the system enters either Mott, or superfluid phase by changing a total number of atoms.

one can look at the expectation values of some operators at the transition point of a $1 \mathrm{D} \mathrm{BH}$ model at $\bar{n}=1$. The ground state wave-function of this system has presumably pronounced deviations from (16). For instance, one obtaines there that (i) the nearest neighbor correlation length function $\left\langle b_{j}^{\dagger} b_{j+1}\right\rangle$ is approximately 0.8 and (ii) the variance of on-site number operator, $\left[\left\langle n_{j}^{2}\right\rangle-\left\langle n_{j}\right\rangle^{2}\right]^{1 / 2}$, equals about 0.6 [190]. These quantities significantly differ from the predictions obtained from the ground state for $t / U=0$ (16). Notice also that they attain substantial values since they are bounded by unity for any $t / U$.

It is interesting to ask what are the critical points $(t / U)_{c}$ in different dimensions, and at different filling factors. Here we list the most accurate estimations up to date for the case $\bar{n}=1$ which has been systematically studied in the past. In one dimension, the critical point was precisely determined by DMRG analysis: $(t / U)_{c} \approx 0.29$ [191]. In two dimensional system, a recent quantum Monte Carlo studies estimate the position of the critical point, $(t / U)_{c}$, to be around 0.061 [192]. In the three dimensional model, the perturbative expansion gives $(t / U)_{c} \approx 0.034$ [193]. The locations of the critical points for different small filling factors in different dimensional lattices were quite accurately calculated in Ref. [193].

- Generic transitions. A quick look at the dotted line in Fig. 3 reveals that the system can cross the superfluid - Mott insulator phase boundary through trajectories that do not correspond to a fixed filling factor. In such a case we say that the system undergoes the generic (density-driven) SF - MI phase transition. This transition does not belong to the universality class of the $X Y$ spin model, thus it is characterized by different critical exponents. In particular, one finds that $z \nu=1$ during generic transitions in $1 \mathrm{D}, 2 \mathrm{D}$ and $3 \mathrm{D}$ systems [30].

Since for this case the number of particles is not conserved, one should add the term $-\mu \sum_{i} n_{i}$ to the Hamiltonian (14), and find a ground state with a number of particles depending on the chemical potential $\mu$. The critical behaviour can be 
observed in at least two quantities: the compressibility $\kappa=\partial \rho / \partial \mu$, (where $\rho$ is atom density), and the superfluid fraction $\rho_{s}$. The first one, diverges as one approaches the Mott lobes from a superfluid side (Fig. 3), while the second goes to zero in this limit. Both the compressibility and the superfluid fraction stay zero inside the lobes.

To illustrate these statements let us consider a one dimensional system, at fixed $t / U$, undergoing a phase transition induced by a change in the number of atoms. In this case, the theory of Fisher et al. [30] predicts that (i) $\kappa \sim|\mu-\tilde{\mu}|^{-\nu(z-1)}$ ( $\tilde{\mu}$ is a chemical potential at phase boundary); (ii) $\rho_{s} \sim|\rho-\tilde{\rho}|^{z-1}$ ( $\tilde{\rho}$ is a filling factor of a Mott lobe approached during transition). The early Quantum Monte Carlo simulations $[194,195]$ have verified these predictions giving the following estimations of the critical exponents: $z \approx 2.04$ and $\nu \approx 0.48$.

Recently, there has been a lot of interest in generalizing MI-SF transition and Bose-Hubbard model to more "exotic" situations, such as atoms in optical cavities [173,174], or $p$ band Hubbard model, where transverse staggered order occurs [196]. In triangular lattices, stripe order was predicted both in SF and MI phases [197].

\section{The Hubbard model: Methods of treatment}

\subsection{Introduction}

In this section we review at a rather basic level some of the standard theoretical tools used in condensed matter theory to treat many body systems of interest. We discuss also novel developments which - taking advantage of quantum information methods provide an efficient way to calculate ground state properties and dynamical evolution of many condensed matter systems. The underlying philosophy of these new methods is closely related to the well-established Density Matrix Renormalization Group (DMRG) method [47, 231], and consist in truncating the dimension of the Hilbert space, which diverges exponentially with the size of the system, to a manageable size, considering entanglement properties of different bipartite partitions.

Analytical and numerical methods often rely on the size and dimensionality of the system. Powerful techniques like bosonization [46,48,232], Bethe ansatz [45, 233, 234], Jordan-Wigner transformation [154,235], or the mentioned DMRG exist and allow to solve some paradigmatic one dimensional systems, such as for instance Heisenberg spin $1 / 2, X X Z$ spin chains, or 1D Hubbard model of strongly interacting electrons. These methods are very well established to treat many body problems in one dimension, but often fail in higher dimensions. Finite size effects are also crucial in the study of strongly correlated systems, because quantum phase transitions occur only in the thermodynamic limit at zero temperature. It is thus important to know how finite size effects affect the statics and dynamics of strongly correlated systems, and the signatures of quantum phase transitions.

We begin this section by focussing on methods of treatment of the ideal homogeneous Bose Hubbard model. As we have discussed in the previous section, this system exhibits a phase transition: the superfluid-Mott insulator (SF-MI) quantum phase transition. Despite its simplicity, the interplay between tunneling and on-site interactions in the $\mathrm{BH}$ model is by no means trivial. Excitations in the limit of small interactions can be described with the help of Bogoliubov transformations. In the strong interaction limit, when the ratio between tunneling and on-site interactions is 
much smaller than one, tunneling can be treated as a perturbation, and the original Hamiltonian can be replaced by an effective Hamiltonian. In some cases, it is also possible to use a mean field approach, like for instance the Gutzwiller ansatz, which assumes that the many body wave functions have a product-over-sites form, and is conceptually and numerically relatively easy to implement. As we shall see, it predicts quite correctly the critical points separating the Mott phase from the SF phase for $3 \mathrm{D}$, or even 2D lattices, but its accuracy decreases dramatically for $1 \mathrm{D}$ systems. We discuss then briefly exact diagonalisation, Quantum Monte Carlo, and phase space methods. Subsequently, we discuss very shortly 1D methods: Bethe ansatz, JordanWigner and bosonisation. We analyze in more detail the novel approach to DMRG provided by Quantum Information. Finaly, we discuss some methods to treat Fermi and Fermi-bose Hubbard models.

\subsection{Weak interactions limit}

Here we want to illustrate how the Bose-Hubbard model can be solved in the limit of small interactions. Our discussion follows Ref. [236], where the Bogoliubov approach was developed.

After adding the $-\mu \sum_{i} n_{i}$ term to Eq.(14), the Hamiltonian can be conveniently written as:

$$
H=-t \sum_{\langle i, j\rangle}\left(b_{i}^{\dagger} b_{j}+\text { h.c. }\right)+\frac{U}{2} \sum_{i} b_{i}^{\dagger} b_{i}^{\dagger} b_{i} b_{i}-\mu \sum_{i} b_{i}^{\dagger} b_{i},
$$

where $\mu$ denotes the chemical potential. We assume a regular $d$-dimensional lattice, consisting on $M$ sites, and the distance between neighboring sites is " $a$ ". In the limit of $t / U \rightarrow \infty$, interactions between atoms are negligible. The system is completely condensed in the ground state, and $N_{0}$ (the number of condensed atoms) equals $N$ (the total number of atoms). When interactions become non-negligible, atoms gradually leave the condensate. To describe this process, it is convenient to work in momentum space: $b_{j}=\frac{1}{\sqrt{M}} \sum_{\vec{k}} \exp \left(-i \vec{k} \vec{x}_{j}\right) a_{\vec{k}}$, where $\vec{x}_{j}$ points into $j$-th lattice site, and $\vec{k}$ is discretised over the first Brillouin zone. Using the identity $\sum_{i} e^{i\left(\vec{k}_{1}-\vec{k}_{2}\right) \vec{x}_{i}}=M \delta_{\vec{k}_{1}, \vec{k}_{2}}$ one obtains:

$$
H=\sum_{\vec{k}}[-\varepsilon(\vec{k})-\mu] a_{\vec{k}}^{\dagger} a_{\vec{k}}+\frac{U}{2 M} \sum_{\vec{k}_{1}, \vec{k}_{2}, \vec{k}_{3}, \vec{k}_{4}} \delta_{\vec{k}_{1}+\vec{k}_{2}, \vec{k}_{3}+\vec{k}_{4}} a_{\vec{k}_{1}}^{\dagger} a_{\vec{k}_{2}}^{\dagger} a_{\vec{k}_{3}} a_{\vec{k}_{4}},
$$

where $\varepsilon(\vec{k})=2 t \sum_{i=1}^{d} \cos \left(k_{i} a\right)$. As $t / U \rightarrow \infty$, the ground state converges towards $\sim a_{0}^{\dagger N}|0\rangle$. The Bogoliubov approach relies on the transformation $a_{0} \rightarrow \sqrt{N_{0}}+a_{0}$, where the new operator $a_{0}$ is responsible for fluctuations of the number of condensed atoms. Substituting the above expression in Eq.(18), one finds, up to the quadratic terms:

$$
\begin{aligned}
H & =N_{0}\left(-z t-\mu+\frac{U}{2} n_{0}\right)+\sqrt{N_{0}}\left(U n_{0}-t z-\mu\right)\left(a_{0}+a_{0}^{\dagger}\right) \\
& +\sum_{\vec{k}}(-\varepsilon(\vec{k})-\mu) a_{\vec{k}}^{\dagger} a_{\vec{k}}+\frac{U n_{0}}{2} \sum_{\vec{k}}\left(4 a_{\vec{k}}^{\dagger} a_{\vec{k}}+a_{\vec{k}} a_{-\vec{k}}+a_{\vec{k}}^{\dagger} a_{-\vec{k}}^{\dagger}\right),
\end{aligned}
$$


where $n_{0}=N_{0} / M$ is the condensate density and $z=2 d$. Setting the chemical potential to $\mu=U n_{0}-z t$ removes the linear part while the quadratic one is diagonalized by the Bogoliubov transformation: $c_{\vec{k}}=u_{\vec{k}} a_{\vec{k}}+v_{\vec{k}} a_{-\vec{k}}^{\dagger}$. Notice that $\left|u_{\vec{k}}\right|^{2}-\left|v_{\vec{k}}\right|^{2}=1$ from the requirement that $\left[c_{\vec{k}}, c_{\vec{k}}^{\dagger}\right]=1$. After a simple algebra, one obtains that within the quadratic approximation the Hamiltonian reduces to:

$$
H=-\frac{U n_{0} N_{0}}{2}+\frac{1}{2} \sum_{\vec{k}}\left(\hbar \omega_{\vec{k}}+\varepsilon(\vec{k})-z t-U n_{0}\right)+\sum_{\vec{k}} \hbar \omega_{\vec{k}} c_{\vec{k}}^{\dagger} c_{\vec{k}},
$$

if

$$
\begin{aligned}
& \left(\left|u_{\vec{k}}\right|^{2}+\left|v_{\vec{k}}\right|^{2}\right)\left[U n_{0}-\varepsilon(\vec{k})+z t\right]-U n_{0}\left(v_{\vec{k}} u_{\vec{k}}^{*}+v_{\vec{k}}^{*} u_{\vec{k}}\right)=\hbar \omega_{\vec{k}}, \\
& \left(u_{\vec{k}}^{2}+v_{\vec{k}}^{2}\right) U n_{0}-2\left[U n_{0}-\varepsilon(\vec{k})+z t\right] v_{\vec{k}} u_{\vec{k}}=0 .
\end{aligned}
$$

Assuming that $u_{\vec{k}}$ and $v_{\vec{k}}$ are real, one easily obtains from these equations:

$$
\begin{aligned}
\hbar \omega_{\vec{k}} & =\sqrt{[z t-\varepsilon(\vec{k})]^{2}+2 U n_{0}[z t-\varepsilon(\vec{k})]} \\
v_{\vec{k}}^{2} & =u_{\vec{k}}^{2}-1=\frac{1}{2}\left(\frac{z t-\varepsilon(\vec{k})+U n_{0}}{\hbar \omega_{\vec{k}}}-1\right) .
\end{aligned}
$$

These results reveal that the excitation spectrum is gapless in the thermodynamic limit $M, N \rightarrow \infty$ at $M / N$ being fixed. Indeed, for the long wavelength (phonon) modes $(|\vec{k}| a \ll 1)$ we find that:

$$
\hbar \omega_{\vec{k}} \approx|\vec{k}| a \sqrt{t} \sqrt{t|\vec{k}|^{2} a^{2}+2 U n_{0}}
$$

i.e., the energy of a single excitation can be arbitrarily small: an expected result showing that the Bogoliubov approach does not work in the Mott phase.

At zero temperature, there are no excitations in the system, so that the ground state is a Bogoliubov vacuum $\mid$ vac $\rangle$, such that $c_{\vec{k}} \mid$ vac $\rangle=0$. At finite temperature, say $T$, excitations are present, and occupations of different modes satisfy $\left\langle c_{\vec{k}}^{\dagger} c_{\vec{k}}\right\rangle=\left[\exp \left(\frac{\hbar \omega_{\vec{k}}}{k T}\right)-1\right]^{-1}$, in accordance with the Bose-Einstein statistics. Using these properties and the solutions (21) and (22) one can easily calculate different quantities (e.g., correlation functions, number of condensed atoms, etc.) both at zero and finite temperatures. It has to be remembered, however, that reliable predictions can be obtained as long as $N_{0}(T) \sim N$, which can be self-consistently verified within this approach. It is also worth to stress that the Bogoliubov approach can be applied to time-dependent problems without further complications. Time dependent Bogoliubovde Gennes method, together with variational approach and the Kibble-Zurek mechanism has been recently used to show the scaling behaviour of the time-dependent correlations [237]. 


\subsection{Strong interactions limit}

Let us now study the Bose-Hubbard model in the limit of stong interactions, i.e., when the system is in the Mott phase. A systematic approach for studies of the Mott insulator phase is provided by the strong coupling expansion, i.e., a perturbative expansion in $t / U$ of Eq.(14).

To perform strong coupling expansion one splits the Hamiltonian into two parts: $H_{0}$, whose eigenstates are exactly known at $t / U=0$, and treat $-t / U \sum_{\langle i, j\rangle}\left(b_{i}^{\dagger} b_{j}+h . c.\right)$ the tunneling part, as a perturbation. Within this approach, the expectation values of some operators are expressed as a series of the form $\sum_{i} a_{i}(t / U)^{i}$. Expansions up to 14 th-order have been calculated, which guarantees in some cases, a high accuracy of perturbative predictions. High order calculations can be performed symbolically on a computer, so that the expansion coefficients $\left(a_{i}\right.$ 's), can be obtained exactly (not as the double precision numbers). The theoretical background for these calculations was set up in $[238,239]$, where this method was applied to spin systems. Below we review the relevant results in the context of the Bose-Hubbard model.

To start with, one can use the strong coupling expansion to determine the phase boundaries on the $(\mu / U, t / U)$ plane (Fig. 3) [191, 193, 240-242]. In this case, the unperturbed bare hamiltonian is $H_{0}=\frac{1}{2} \sum_{i} n_{i}\left(n_{i}-1\right)-\mu / U \sum_{i} n_{i}$, and one calculates perturbatively (i) the energy of the ground state with exactly $\bar{n}$ atoms per site; (ii) the ground state energy of the system with one particle added (subtracted) to (from) the system with filling factor $\bar{n}$. Setting the energy difference between (i) and (ii) cases to zero, one obtains the value of the chemical potential at the upper (lower) boundary between insulator and superfluid phases. These calculations can be performed for any dimensional lattice, and the order of expansion can be as large as 13th [240]. For one dimesional systems [191,242], the predicted structure of Mott insulator lobes is in a very good agreement with the numerical results obtained via DMRG calculations. The perturbative expansions can also be used together with different extrapolation methods leading to the determination of the critical exponents $z$ and $\nu[193,240]$.

Very recently this method has been applied to calculate the lobes for a modified 1D Bose-Hubbard model describing atoms in an optical lattice created by pumping a laser beam into the cavity [173]. The major difference to the standard case is that the intensity of the cavity field depends on the number of atoms present, since the atoms shift collectively the cavity resonance. In effect the coefficients $t$ and $U$ become very complex functions of all of the relevant parameters; cavity detuning, intensity of the pumping laser, $N$, etc. Moreover, quantum fluctuations of the resonance shift induce long range interactions between the atoms. The phase diagram, as a function of the dimensionless parameters $\mu / U$ and $\kappa / \eta$, where $\kappa$ is the cavity width and $\eta$ is the pumping laser strength, is shown in Fig. $4 .\left(1 / \sqrt{n_{p h}}=\kappa / \eta\right.$, where $n_{p h}$ is the number of photons in the cavity.) The striking effect is the overlap of different Mott phases, which is the consequence of the fact that the expressions for $t$ and $U$ for $n_{0}=1,2,3, \ldots$ Mott phases are different.

The strong coupling expansion has also been used to calculate the correlation functions $\left\langle b_{i}^{\dagger} b_{i+j}\right\rangle$ and the structure factor $[190,240]$. A typical prediction of the strong coupling expansion reads as

$$
\left\langle b_{i}^{\dagger} b_{i+1}\right\rangle=4 \frac{t}{U}-8\left(\frac{t}{U}\right)^{3}-\frac{272}{3}\left(\frac{t}{U}\right)^{5}+\frac{20272}{81}\left(\frac{t}{U}\right)^{7}-\frac{441710}{729}\left(\frac{t}{U}\right)^{9}
$$




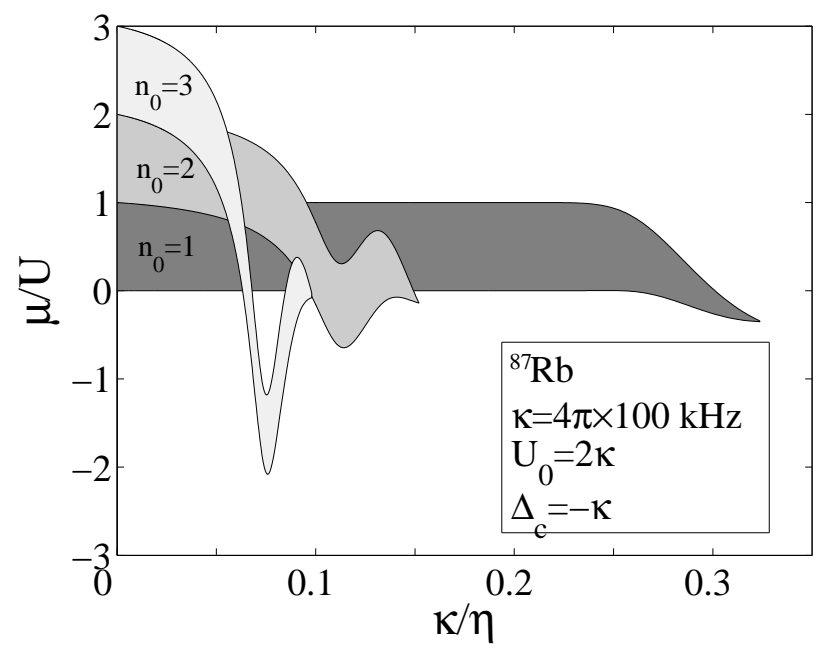

Figure 4. Overlapping MI phases for the Bose-Hubbard model in an optical cavity (from [173]).

$$
+\frac{39220768}{2187}\left(\frac{t}{U}\right)^{11}+\frac{8020902135607}{94478400}\left(\frac{t}{U}\right)^{13}+\mathcal{O}\left((t / U)^{15}\right),
$$

which was obtained in a one dimensional system at unit filling factor in [190]. The differences between such analytical result and the numerical calculation turn out to be hardly visible for $t / U<0.3$, i.e., in entire Mott phase. The strong coupling expansion has been also employed to determine density-density correlations, $\left\langle n_{i} n_{i+j}\right\rangle$, and the variance of on-site atom occupation $\left[\left\langle n_{i}^{2}\right\rangle-\left\langle n_{i}\right\rangle^{2}\right]^{1 / 2}[190]$. All these quantities should be directly measurable in an ongoing experiment in a homogeneous, one dimensional lattice [243].

\subsection{The Gutzwiller mean-field approach}

The Gutzwiller mean-field approach [29, 244,245] has been used in numerous papers devoted to the Bose-Hubbard model. In its simplest version, it is based on the approximation of the many-body wave function by the product over single site contributions

$$
|\Psi\rangle=\prod_{i} \sum_{n=0}^{n_{\max }} f_{n}^{(i)}|n\rangle_{i}
$$

where $|n\rangle_{i}$ denotes the Fock state of $n$ atoms in the $i$-th lattice site, $n_{\text {max }}$ is a system size-independent cut off in the number of atoms per site, and $f_{n}^{(i)}$ corresponds to the amplitude of having $n$ atoms in the $i$-th lattice site. The amplitudes are normalized to $\sum_{n}\left|f_{n}^{(i)}\right|^{2}=1$.

To see what Gutzwiller approach predicts for quantum phase transitions, we focus now on the case of an homogeneous system having an integer number of particles 
per site $\bar{n}$. In this case, one obtains that for $t / U<(t / U)_{g}$, where $(t / U)_{g}$ denotes a critical value, the Gutzwiller amplitudes are $f_{n}^{(i)}=\delta_{n \bar{n}}$, so that the wave function becomes a single Fock state (16). Therefore, the Gutzwiller wave function exactly reproduces the system wave function when $t / U \rightarrow 0$. It is also possible to argue that the differences between exact result and (23) are negligible in the limit of a large lattice and $t / U \rightarrow \infty$ [246]. Therefore, the common expectation is that (23) reasonably interpolates between superfluid and Mott insulator limits, which we discuss below pointing out the advantages and disadvantages of the Gutzwiller method.

The critical point according to the Gutzwiller approach, $(t / U)_{g}$, is located at $\frac{1}{5.8 z}$ for $\bar{n}=1$ [246], where $z$ is the number of nearest neighbors. Comparing this result to the more reliable findings from Sec. 2.5, one observes that the agreement improves with the system dimensionality: the Gutzwiller result is poor for a 1D system $(z=2)$, and satisfactory for a $3 \mathrm{D}$ one $(z=6)$.

The Gutzwiller method is quite straightforward in the numerical implementation. In the static case the amplitudes $f_{n}^{(i)}$ are real numbers and can be found by minimization of $\left\langle\Psi\left|H-\mu \sum_{i} n_{i}\right| \Psi\right\rangle$, where $H$ is given by (14), and $\mu$ is a chemical potential used to enforce a desired number of atoms in the ground state. The minimization can be done in a standard way, e.g. with the conjugate gradient method [247], and faces no problems as long as the system is homogeneous, or the external potential imposed on it is quite regular, e.g. harmonic. In the later case, a calculation in an experimentally realistic 3D configuration consisting of $65^{3}$ lattice sites has been recently done [248].

The extension of the Gutzwiller approach to time-dependent problems is simple [139]. (Alternative dynamical mean field approach has been formulated in Refs. [249].) Indeed, the stationarity of $\left\langle\Psi\left|i \frac{d}{d t}-H\right| \Psi\right\rangle$, where $H$ is given by (14), leads to the equation

$$
i \frac{d}{d t} f_{n}^{(i)}=\frac{U}{2} n(n-1) f_{n}^{(i)}-t \Phi_{i}^{\star} \sqrt{n+1} f_{n+1}^{(i)}-t \Phi_{i} \sqrt{n} f_{n-1}^{(i)},
$$

where $\Phi_{i}=\sum_{\langle i, j\rangle}\left\langle b_{j}\right\rangle=\sum_{\langle i, j\rangle} \sum_{n} \sqrt{n} f_{n-1}^{(j) \star} f_{n}^{(j)}$ (the first sum goes over all $j$ being nearest neighbors of $i$ ). Numerical integration of (24) is straightforward.

One should also appreciate the simplicity of Gutzwiller approach extensions to different systems, e.g., mixtures of bosonic gases [139,140], Bose-Fermi mixtures [87, 183, 210], etc. Particularly interesting are extended Hubbard models such as those involving dipolar interactions, where one expects the appearance of supersolid and checkerboard-like phases at low filling factors [147, 178, 250]. Despite all these nice features, there are also problems associated with the Gutzwiller ansatz.

The picture that the Mott insulator corresponds to a single Fock state (16) for the filling factor $\bar{n}$ is obviously incorrect - see a more detailed discussion of the Mott phase in Sec. 2.5. Another drawback of (23) is that the correlation functions between different sites factorize into products of single site contributions, e.g., $\left\langle\Psi\left|b_{i}^{\dagger} b_{j}\right| \Psi\right\rangle=$ $\left\langle\Psi\left|b_{i}^{\dagger}\right| \Psi\right\rangle\left\langle\Psi\left|b_{j}\right| \Psi\right\rangle$ for $i \neq j$. As a result, there is lack of dependence of correlations on the distance between lattice sites. Also, formula (23) does not correspond to a well defined number of particles. This problem can be solved by a proper projection of the wave function (23) onto the subspace with fixed number of atoms [139,244], but the subsequent calculations become complicated. Finally, the Gutzwiller approach underestimates finite size effects: it predicts a "quantum phase transition" in systems of any size due to decoupling into the product of single site contributions (23). The 
true quantum phase transition, however, requires a large system.

The performance of the Gutzwiller ansatz can be, to a limited extent, perturbatively improved [251]. These corrections significantly modify the Gutzwiller wave function for $t / U$ smaller than $(t / U)_{g}$. As a result, both the variance of an on-site atom occupation and the correlation functions $\left\langle b_{i}^{\dagger} b_{j \neq i}\right\rangle$ become nonzero for $t / U>0$, which is a progress with respect to the traditional Gutzwiller approach.

Finally, we mention that the Gutzwiller approach can be supplemented by other mean-field-like calculations exploring the properties of Green functions [252-257]. They predict virtually the same transition points in different dimensions as the Gutzwiller method, but allow for determination of the excitation spectra and finite temperature calculations.

\subsection{Exact diagonalizations}

Exact diagonalizations of the Bose-Hubbard model can be done for small systems only. The problems result from the enormous size of the Hilbert space, given by

$$
\mathcal{H} \mathcal{S}(N, M)=\frac{(N+M-1) !}{N !(M-1) !}
$$

where $N$ and $M$ stand for the number of atoms and the number of lattice sites, respectively. To illustrate predictions of $(25)$ we consider the simplest system undergoing a quantum phase transition in the thermodynamical limit, i.e., the $M=N$ case. For instance, for $N=8,10,12$ one obtains $\mathcal{H} \mathcal{S}(N, N)=6435,92378,1352078$ respectively. Moreover, one can easily verify that

$$
\frac{\mathcal{H} \mathcal{S}(N+1, N+1)}{\mathcal{H} \mathcal{S}(N, N)} \stackrel{N}{\approx}^{1} 4
$$

which quantifies the fast increase of the Hilbert space size with system size. This shows that a standard diagonalization where all matrix elements are stored, and no symmetries are employed, can face problems already for $N=M>8$. Additionally, from the exponential increase of the Hilbert space size with the system size, any significant progress due to improvement of computer resources is unlikely. To overcome to some extend the above limitations, one can take into account the following. First, one can use numerical routines that store non-zero matrix elements only, e.g., ARPACK [258]. Then, diagonalization of $N=M=12$ system faces no problems on a computer with about $1 \mathrm{~Gb}$ of memory provided that one looks for a limited number of eigenstates instead of the full spectrum. Second, one can cut the Hilbert space by restricting maximal site occupation to $K$ atoms. Such a choice can be justified by a quadratic increase of the interaction energy with the site occupation number and is present in DMRG and Quantum Monte Carlo schemes. The size of the Hilbert space (see Appendix B) is then

$$
\left.\mathcal{H} \mathcal{S}(N, M)\right|_{K}=\sum_{j=0}^{\left[\frac{N}{K+1}\right]}(-1)^{j}\left(\begin{array}{c}
M+N-1-j(K+1) \\
M-1
\end{array}\right)\left(\begin{array}{c}
M \\
j
\end{array}\right)
$$


where $[x]$ stands for the largest integer number not greater than $x$. For $K=N$, (26) reduces to (25). A typical choice leading to well converged results in a wide range of Bose-Hubbard model parameters is $K=4[186,242]$. It results in the size of Hilbert space equal to $5475,72403,975338$ for $N=8,10,12$, respectively. These numbers suggest that rather a slight progress can be achieved by cutting the Hilbert space size this way. The most powerful simplifications are possible when the problem under consideration has some symmetries, i.e., when there exists a set of operators $\left\{O_{i}\right\}$ commuting with the Hamiltonian. Then, one can split a Hilbert space into subspaces composed of states with well defined eigenvalues of the $O_{i}$ operators. In this way, the problem of diagonalization of the full Hamiltonian reduces to a few independent diagonalizations of smaller matrices. It allows for reduction of memory requirements and helps in getting a relatively large number of excited eigenstates. An example relevant for this review can be found in $[110,111]$, where a system of cold fermions placed in a kagomé lattice is considered. There exists a translational symmetry generated by an operator having $S$ different eigenvalues. The Hilbert space splits into $S$ subspaces, and diagonalization of the full Hamiltonian, say $L \times L$ matrix, reduces to $S$ independent diagonalizations of $\sim L / S \times L / S$ matrices. A more detailed description of exact diagonalization procedures for many-body quantum systems can be found in [259].

\subsection{Quantum Monte Carlo}

The Quantum Monte Carlo (QMC) method was successfully used for studies of the Bose-Hubbard model [194,195, 260-262], after publication of the seminal paper of Fisher et al. [30]. The computer resources and QMC algorithms allowed, at that time, to consider systems of the size of a few tens of lattice sites/atoms in one and two dimensional models. Nowadays, systems composed of $10^{3}$ sites and atoms in any dimension can be routinely studied. It allows to consider 3D configurations, which are quite realistic from an experimental perspective $[192,263]$. The progress comes obviously from better computer resources and more efficient algorithms (see e.g., $[109,264,265])$. Interestingly, efficient numerical codes for QMC simulations are now publicaly available [266].

The QMC approach allows to calculate different properties of a system being in equilibrium at finite temperature. During studies of the Bose-Hubbard model these temperatures can be chosen so low that the simulation describes essentially the zerotemperature physics of the system. The quantities usually calculated within the QMC approach are: the superfluid fraction, the chemical potential $(\mu)$, the density of atoms $(\rho)$, the variance of the on-site occupation, and the compressibility $(\kappa=\partial \rho / \partial \mu)$.

Early QMC calculations [194, 195, 260-262] focused on determination of the phase diagram in the $(\mu / U, t / U)$ plane (Fig. 3), and on the influence of disorder on it. In particular, they provided estimations of the position of the critical points, and they have verified critical behaviour of different quantities, e.g., scaling properties of compressibility and superfluid fraction, in the neighbourhood of the phase boundary, predicted in [30] (see Sec. 2.5).

Among the recent QMC studies of the Bose-Hubbard related to physics of cold atoms in optical lattices we would like to focus on investigations of harmonically trapped systems $[192,267-269]$. Below we briefly review findings of these works, stressing the differences between the harmonically trapped model and the homogeneous one. 
The Bose-Hubbard Hamiltonian in the presence of the harmonic trap takes the form (14) with an additional term $-\sum_{i} \mu_{i}^{\text {local }} n_{i}$. The $\mu_{i}^{\text {local }}=\mu-V r_{i}^{2}$ is a local chemical potential, $r_{i}$ is the distance from the trap centre, and $V$ is the strength of a harmonic trap. The state diagram for such a problem in one dimension was calculated in [267]. In a generic situation one finds that there are plateaus, characterized by integer density of atoms and small atom number fluctuations, surrounded by regions of space where atom number fluctuations are large and density is non-integer. The former (latter) are identified as Mott insulator (superfluid) domains. Amazingly, images of these domain shells have been recently observed experimentally [270,271]. It should be stressed, however, that there are significant differences between superfluid and Mott insulator "phases" in harmonically trapped and homogeneous models.

The harmonically trapped system is gapless even in the presence of Mott domains [268]. It is also always globally compressible [267]. Both these properties are in a striking difference to the homogeneous case. Inhomogeneity of a trapped system suggests that the critical behaviour might be recovered in local quantities, e.g., the local compressibility $\kappa_{i}^{\text {local }}=\partial\left\langle\sum_{j} n_{j}\right\rangle / \partial \mu_{i}^{\text {local }}$ (see $[192,272]$ for a systematic discussion in both harmonic and quartic trapping potentials). This quantity, however, does not show the critical behaviour at the border between Mott and superfluid domains. All this leads to the conclusions that: i) in a harmonically trapped system, instead of a true quantum phase transition, there is rather a crossover, and ii) it will be very interesting to perform the experiments in homogeneous systems in a "box" potential. Such potentials are currently being realized, for instance, by the group of M. Raizen [243].

Another difference between homogeneous and trapped models is observed in the visibility of interference patterns measured after releasing the atoms from the external potentials [269,273]. While in a homogeneous system the visibility of interference fringes is a smooth monotonic function of $t / U$ ratio, in the trapped model one finds both kinks (sudden changes of the slope) and non-monotonic behaviour caused by the presence of correlations between disconnected superfluid domains.

\subsection{Phase space methods}

Phase space methods have been introduced to quantum mechanics relatively early by Wigner [274,275] and Moyal [276], but their rapid development started in the 60-ties with the applications to quantum optics by Glauber [3] and Sudarshan [277]. To a great extent, quantum optical studies of quasi-probability distributions, such as Wigner functions, or $P$ - or $Q$ - distributions, contributed enormously to our modern understanding of quantum noise $[6,7]$. These methods usually map the density matrix of the considered system onto a function fulfilling a generalized Fokker-Planck type of equations, and try to replace this equation by systems of Langevin-like equations that can be simulated using classical Monte Carlo methods. The necessary condition is that the corresponding quasi-probability, or phase space quasi-distribution must be a reasonable probability measure, which not always is the case. Glauber-Sudarshan $P$-representation often is a highly singular distribution, and Wigner function might take negative values.

All these problems can be sometimes overcome, and several groups have started to use phase space methods to simulate many body problems. Pioneering work in this direction for bosonic gases has been done by P. Drummond's group [278]. These methods were extended to fermions in Ref. [279]. The authors study various signatures 
of strongly correlated ultra-cold fermions in optical lattices, performing collective mode calculations, where a sharp decrease in collective mode frequency is predicted at the onset of the Mott metal-insulator transition. They have also looked at correlation functions at finite temperatures, using a new exact method that applies the stochastic gauge technique with a Gaussian operator basis.

Somewhat similar approach has been developed by Y. Castin group [280]. This approach allowed, for instance, for precise determination of the fluctuations of the number of condensed atoms in an interacting Bose gas. It has been recently extended to describe lattice Hubbard problems [281], and to Fermi gases [282, 283].

It is worth stressing that stochastic, or phase space methods become very efficient when the fluctuations become classical, i.e. at high, or at least moderate high temperatures, which for the BEC might mean 0.1-0.2T . Truncated Wigner approach, or simulations of the Gross-Pitaevskii equations with random initial conditions, that mimic the initial thermal equilibrium are easy to implement and very accurate. Several groups have used such approach with great success. Hannover group used such method to describe phase fluctuations in quasi-1D BEC's [284-287]. Barcelona-Hamburg collaboration applied these approach to study dynamics of spinor condensates [288].

The so-called classical field method was developed by K. Burnett [289] and K. Rzążewski [290] groups. Both groups put some emphasis on understanding the concept of temperature in the microcanonical ensemble, whereas the latter recently realized the modeling of the decay of unstable vortex states.

\section{8 $1 D$ methods}

In this section we give a very short guide of $1 \mathrm{D}$ methods, illustrating the subsequent steps from Bose-Hubbard model to Luttinger liquid theory. We start by analyzing the Bose-Hubbard Hamiltonian in 1D (Eq. (12)), i.e. a chain of sites with open ends and we perform then the following steps:

a. Hard core bosons. In this limit (i.e. when $U \gg t, \mu$ ), as descibed in Section 2.3, the Bose Hubbard hamiltonian (Eq. (12)) reduces to the XY model in a transverse field (Eq. (13)).

b. Jordan-Wigner transformation. Jordan-Wigner transformation is a way to "fermionize" the, otherwise, bosonic system [154]. One defines:

$$
\begin{aligned}
f_{i} & =\prod_{j<i} \sigma_{z, j} \sigma_{i}, \\
f_{i}^{\dagger} & =\prod_{j<i} \sigma_{z, j} \sigma_{i}^{\dagger},
\end{aligned}
$$

so that $f_{i}^{\dagger} f_{i}=\sigma_{i}^{\dagger} \sigma_{i}=\left(1+\sigma_{z, i}\right) / 2$. It is easy to check that such nonlocal operators are fermionic, i.e.

$$
\left\{f_{i}, f_{j}\right\}=\left\{f_{i}^{\dagger} f_{j}^{\dagger}\right\}=0, \quad\left\{f_{i} f_{j}^{\dagger}\right\}=\delta_{i j}
$$


The inverse relations are also simple:

$$
\begin{aligned}
\sigma_{i} & =\prod_{j<i}\left(2 f_{i}^{\dagger} f_{i}-1\right) f_{i}, \\
\sigma_{i}^{\dagger} & =\prod_{j<i}\left(2 f_{i}^{\dagger} f_{i}-1\right) f_{i}^{\dagger} .
\end{aligned}
$$

With this transformation the Hamiltonian becomes the one of "spinless" fermions:

$$
H=+t \sum_{\langle i\rangle}\left[f_{i}^{\dagger} f_{i+1}+\text { h.c. }\right]-\mu \sum_{i} f_{i}^{\dagger} f_{i},
$$

and the model becomes exactly solvable. Had we started from an extended Hubbard model with, say, nearest neighbour interactions of the form $V \sum_{i} \sigma_{i}^{\dagger} \sigma_{i+1}^{\dagger} \sigma_{i+1} \sigma_{i}$, we would end up with "spinless" interacting fermions with the term $V \sum_{i} f_{i}^{\dagger} f_{i+1}^{\dagger} f_{i+1} f_{i}$.

Jordan-Wigner transformation is essentially a $1 \mathrm{D}$ transformation. There were very many attempts of generalizing it to higher dimension (for a review see [235], for a discussion in the context of applications for atoms see [183]). Only recently, new ideas related to the concept of PEPS, allowed Verstraete and Cirac to propose an interesting efficient generalization of the Jordan-Wigner transformation to 2D [291].

c. Bosonisation, and Luttinger liquid theory. Let us consider now the interacting "spinless" Fermi gas, and try to formulate the low energy effective theory for this model. We assume that fermions do not fill the lowest band, so that in the absence of interactions, the Fermi level is somewhere in the middle of the band. We expect that at low temperatures interesting physics will occur close to the Fermi energy. It is thus reasonable to linearize the fermionic dispersion relation at the Fermi energy, $\epsilon(k)=\epsilon\left( \pm k_{F}\right) \pm c_{F}\left(k \pm k_{F}\right)$, with the "sound velocity" $c_{F}=d \epsilon(k) /\left.d k\right|_{k_{F}}$. In 1D there are two values of momenta $\pm k_{F}$ where the Fermi energy is reached: one corresponds to left, and another to right going fermions. Note that all of the interesting low energy physics happens close to the Fermi surface (points). That implies that the states with momenta far from $\pm k_{F}$ will practically never participate in any relevant physical process, and will remain deep in the filled Fermi sea. It is thus reasonable treat the left and right going fermions close to $\pm k_{F}$ as independent and introduce two fermionic species, described in the momentum representation by $L(k), L^{\dagger}(k)$, and $R(k), R^{\dagger}(k)$, respectively; $k$ is here momentum relative to $\pm k_{F}$, so that it attains values from $-\infty$ to $+\infty$. The Hamiltonian, becomes

$$
\begin{aligned}
H & =+\sum_{k}\left[-c_{F}(k) L^{\dagger}(k) L(k)+c_{F}(k) R^{\dagger}(k) R(k)\right] \\
& +\frac{1}{L} \sum_{k_{1}, k_{2}, q} V(q) L^{\dagger}\left(k_{1}-q\right) R^{\dagger}\left(k_{2}+q\right) R\left(k_{2}\right) L\left(k_{1}\right) .
\end{aligned}
$$

This is a Luttinger model $[46,292]$. One introduces now the operators

$$
\rho_{L}(q)=\sum_{k} L^{\dagger}(k+q) L(k)
$$




$$
\begin{aligned}
& \rho_{L}(-q)=\sum_{k} L^{\dagger}(k) L(k+q)=\rho_{L}^{\dagger}(q), \\
& \rho_{R}(q)=\sum_{k} R^{\dagger}(k+q) R(k), \\
& \rho_{R}(-q)=\sum_{k} R^{\dagger}(k) R(k+q)=\rho_{L}^{\dagger}(q),
\end{aligned}
$$

and observes that $\left[\rho_{R}(-q), \rho_{R}\left(+q^{\prime}\right)\right]=\sum_{k_{F}-q^{\prime}}^{k_{F}} R^{\dagger}\left(k+q-q^{\prime}\right) R(k)$. In the LuttingerTomonaga approximation [292] one replaces now these and similar commutators by their averaged values over the ideal Fermi sea. The result is

$$
\left[\rho_{R}(-q), \rho_{R}\left(+q^{\prime}\right)\right]=\frac{q L}{2 \pi} \delta\left(q-q^{\prime}\right)
$$

implying that for $q>0$, we can introduce $b_{q}^{\dagger}=\rho_{R}(q) \sqrt{2 \pi / q L}, b_{q}=\rho_{R}(-q) \sqrt{2 \pi / q L}$, which may be regarded as bosonic annihilation and creation operators, respectively, since $b_{q}, b_{q^{\prime}}^{\dagger}=\delta\left(q-q^{\prime}\right)$. Similar construction is done for the operators $c_{-q}^{\dagger}=\rho_{L}(-q) \sqrt{2 \pi / q L}$, and $c_{-q}=\rho_{L}(q) \sqrt{2 \pi / q L}$. These operators have the interpretation that $b_{q}^{\dagger}$ takes a particle from state $k$ and puts it into $k+q$. It creates thus fermion-hole pairs when $k<k_{F}$ and $k+q>k_{F}$. Analogously, the operator $c_{-q}^{\dagger}$ takes a fermion from an unoccupied state $-k_{F}<k+p$ to the unoccupied state $k<-k_{F}$. The whole Hamiltonian becomes

$$
H=\sum_{q>0} q c_{F}\left[b_{q}^{\dagger} b_{q}+c_{-q}^{\dagger} c_{-q}\right]+\frac{1}{2 \pi} \sum_{q>0}\left[V(q) c_{-q}^{\dagger} b_{q}^{\dagger}+V(q)^{*} b_{q} c_{-q}\right] .
$$

The problem has been thus reduced to an exactly solvable system of interacting harmonic oscillators, describing linear 1D hydrodynamics of a, so called, Luttinger liquid. Bosonisation theory [46] assures that such description can be found for most of the 1D bosonic and fermionic systems with local interactions. For the very recent and very complete description of the interacting Bose gases in quasi-one dimensional optical lattices see the review article of Cazalilla et al. [293].

\subsection{Bethe ansatz}

Bethe ansatz is an analytical method to find exact eigenstates and eigenvalues of some strongly correlated one-dimensional models (although some times it has to be complemented with numerical analysis). By exploiting appropriately the symmetries involved in the Hamiltonian it is possible to diagonalise the Hamiltonian exactly. However, its power relies in the fact that Bethe ansatz characterises all eigenstates by a set of quantum numbers which enumerate the states, according to their physical properties. This method was originally developed by Bethe in 1931 [294] to solve a 1D array of electrons with uniform next neighbour interactions, i.e., spin $1 / 2$ Heisenberg model:

$$
H=-J \sum_{i=1}^{N} \sigma_{\mathbf{i}} \cdot \sigma_{\mathbf{i}+\mathbf{1}}
$$


where $\sigma=\left(\sigma_{x}, \sigma_{y}, \sigma_{z}\right)$ are the standard Pauli matrices and $\mathrm{N}$ denotes the number of sites. The parametrisation of the eigenvectors, i.e. the Bethe ansatz has become a fundamental tool with which many other 1D quantum systems have been shown to be solvable. From the eigenvectors one can compute easily the quantities of interest by calculating the expectation values of the desired operators. In what follows, we summarize the original formulation of Bethe following the pedagogical work of Karbach et. al. [295, 296]. Since, the Heisenberg Hamiltonian commutes with the total spin along the z-direction $\sigma_{T}^{z}=\sum_{i} \sigma_{i}^{z},\left[H, \sigma_{T}^{z}\right]=0$, then:

$$
H=-J \sum_{i=1}^{N}\left[\frac{1}{2}\left(\sigma_{i}^{+} \sigma_{i+1}^{-}+\sigma_{i}^{-} \sigma_{i+1}^{+}\right)+\sigma_{i}^{z} \sigma_{i+1}^{z}\right] \text {. }
$$

Thus, eigenstates of $\sigma^{z}$ are also eigenstates of $H$. Bethe ansatz is, as discussed below, a basis transformation.

We denote by $|0\rangle=|\uparrow\rangle$ the eigenstate of $\sigma^{z}$ with value $+1 / 2$ and by $|1\rangle=|\downarrow\rangle$ the eigenstate with eigenvalue $-1 / 2$. Sorting the basis according to the quantum number $N / 2-r$, where $r$ is the number of flipped spins, is all that is needed to block diagonalise the Hamiltonian. The block $r=0$ corresponds to a single state with all spins up which we call ferromagnetic: $H|F\rangle=H|\uparrow \ldots \uparrow\rangle=-J N / 4$. The case $r=1$ (one spin down) has $N$ invariant vectors, which are labeled by the position of the flipped spin: $|n\rangle=\sigma_{n}^{-}|F\rangle$. To diagonalise this block, which has size $N \times N$, we take into account that the Hamiltonian possesses translational symmetry. Therefore a translational invariant basis can be constructed in the subspace with $r=1$,

$$
|\psi\rangle=\frac{1}{\sqrt{N}} \sum_{n=1}^{N} e^{i k n}|n\rangle,
$$

with wave numbers $k=2 \pi m / N, m=0,1, \ldots N-1$. The vectors $|\psi\rangle$ correspond to a complete spin alignment of the ferromagnetic ground state $|F\rangle$ which is periodically disturbed by a spin wave with wavelength $\lambda=2 \pi / k$. These states are called magnons, or spin-waves.

The subspace $r=2$ cannot be solved by applying any further symmetry and here is where the full power of the Bethe ansatz appears. Solutions (42), for $r=1$, can be also obtained starting from the following ansatz

$$
|\psi\rangle=\sum_{n=1}^{N} a(n)|n\rangle
$$

and finding the values of the coefficients $a(n)$ that satisfy the eigenvalue equation $H|\psi\rangle=E|\psi\rangle$ using periodic boundary conditions. If one applies the same procedure to the subspace $r=2$, i.e. one looks for the coefficients $a\left(n_{1}, n_{2}\right)$ that determine the eigenstates, one finds:

$$
|\psi\rangle=\sum_{1 \leq n_{1}<n_{2} \leq N}^{N} a\left(n_{1}, n_{2}\right)\left|n_{1}, n_{2}\right\rangle .
$$

There are $N(N-1) / 2$ eigenstates. These are characterised by pairs of Bethe (integer) quantum numbers $\lambda_{1}, \lambda_{2}$, which define the "momenta" via the relations $N k_{1}=2 \pi \lambda_{1}+$ 
$\theta, N k_{2}=2 \pi \lambda_{2}-\theta$, such that

$$
a\left(n_{1}, n_{2}\right)=e^{i\left(k_{1} n_{1}+k_{2} n_{2}+\theta_{12} / 2\right)}+e^{i\left(k_{1} n_{2}+k_{2} n_{1}+\theta_{21} / 2\right)},
$$

whereas the angles $\theta_{12}=-\theta_{21}=\theta$ fulfill $2 \cot (\theta / 2)=\cot \left(k_{1} / 2\right)-\cot \left(k_{2} / 2\right)$. Depending on the relative values of the pair $\lambda_{1}, \lambda_{2}$, different type of solutions exist. In general, the Bethe ansatz for an unrestricted number $r$ of flipped spins reads:

$$
|\psi\rangle=\sum_{1 \leq n_{1} \leq \ldots \leq n_{r} \leq N}^{N} a\left(n_{1} \ldots n_{r}\right)\left|n_{1} \ldots n_{r}\right\rangle
$$

and the eigenvectors span a subspace of dimension $N ! /(N-r) ! r$ !. The coefficients $a\left(n_{1} \ldots n_{r}\right)$ have an analogous form to $(45)$, and a generalised solution of the resulting transcendental equations can be easily found numerically.

It is also worth to mention that the Bethe ansatz can be applied to the one dimensional Bose-Hubbard model - see [187] for a complete discussion. In this case, however, the method faces fundamental problems since one has to assume that the maximal occupation of each lattice site is not larger than 2 . As a result, the ansatz is never exact though it gives some valuable and correct, predictions, e.g., vanishing superfluid fraction in the Mott phase.

\subsection{A quantum information approach to strongly correlated systems}

The density matrix renormalisation group (DMRG) $[47,231]$ is a variational method that has had an enormous success in describing ground states of some strongly interacting 1D systems with rather modest computational effort. The underlying philosophy of all DMRG oriented algorithms is that many body systems can be treated almost "exactly" if one is able to truncate the full Hilbert space by removing the degrees of freedom that are not involved neither in the ground state, nor in the dynamical evolution of the system. The difficulty and glory of the method relies on how reliable the truncation is done. Very recently [297-301], quantum information theory has provided a new perspective on the following questions: (i) how to perform an efficient truncation of the Hilbert space, (ii) which quantum systems can be efficiently simulated, (iii) how to simulate dynamical evolutions of strongly correlated systems, (iv) how and when DMRG-oriented methods can be implemented to investigate ground states of $2 \mathrm{D}$ and 3D systems, (v) how classical concepts like correlation length, which diverge on the critical points is linked to entanglement [301,302], etc. In general, this approach is shedding new light in our understanding of complex many body physics. Anders et al. have recently proposed an approximate method to calculate ground states of quantum many-body systems, based on quantum information concepts of "weighted graph states" [303].

Real space renormalisation methods are iterative methods to describe accurately mainly $1 \mathrm{D}$ many body systems. The starting point is a small $1 \mathrm{D}$ chain system which can be exactly diagonalised. Then, the size of the chain is iteratively increased by adding a new lattice site at each step. The Hamiltonian that coupled the new site to the chain is renormalized by disregarding all physically irrelevant couplings and the resulting Hamiltonian diagonalised.

There are, at least, 3 different approaches to perform the truncation [47]: 
- By optimizing expectation values [304]. In this approach the value of some bounded operator (energy, magnetization or density) determines which are the most relevant states contributing to it.

- By optimizing the wave function [231]. The truncation is done such that the renormalized wave function minimizes the Schmidt norm distance to the exact one, i.e. $\| \Psi\rangle-|\Psi\rangle_{D M R G} \|^{2} \rightarrow 0$

- By optimizing entanglement properties. It was shown by G. Vidal [297] that the efficiency in simulating many body systems is directly related to their entanglement behaviour. Efficient simulation is possible if the entanglement of a subsystem with respect to the whole is bounded, or grows at most logarithmically with its size. Also, if entanglement grows linearly in time and block size, simulation of time evolution may be not efficient even in some non-critical 1D systems [299].

3.10.1 Vidal's algorithm. In the following we briefly review the algorithm of G. Vidal [297] (also called Time Evolving Block Decimation (TEBD)) to calculate ground states and dynamics using the Schmidt decomposition to truncate the Hilbert space. Implementing operations on individual sites and on 2 neighboring sites requires only local updating of the expansion, rending the calculations of ground states, expectation values, and 2-body correlations efficient. We also comment how the expansion is linked to the Matrix Product states (MPS) ansatz used in DMRG, and why MPS describe so well ground states of quantum spin systems [300].

Assume that our system consists of a set of atoms loaded in a 1D optical lattice with $M$-sites, whose Hamiltonian is well described by a Bose-Hubbard Hamiltonian (see Eq. (14)) and we are seeking for the ground state $|\Psi\rangle$ of the system. We choose as ansatz the most general description of a state in such Hilbert space which is given by

$$
|\Psi\rangle=\sum_{i_{0}=0}^{d} \ldots \sum_{i_{M}=0}^{d} C_{i_{1} \ldots i_{M}},\left|i_{1}\right\rangle \otimes \ldots\left|i_{M}\right\rangle
$$

where $\left\{\left|i_{j}\right\rangle\right\}$ denotes an orthonormal basis, $M$ is the number of sites, $N$ is the number of atoms, and $d$ indicates the maximal occupation number of site $j$ (maximal $\left.\left\langle a_{j}^{\dagger} a_{j}\right\rangle\right)$. Note that by taking $d=N$, we can be sure that Eq. (47) gives an exact representation of the state. Let us now split the $1 \mathrm{D}$ chain into two blocks: block A, which consists of a single site, and block $\mathrm{B}$, consisting of the remaining $M-1$ sites. For this (or any other bipartite) splitting $(A, B)$, there exist always a bi-orthonormal basis denoted by $\left|\Phi_{\alpha}^{[A]}\right\rangle$ and $\left|\Phi_{\alpha}^{[B]}\right\rangle$, respectively such that:

$$
|\Psi\rangle=\sum_{\alpha}^{\chi_{A}} \lambda_{\alpha}\left|\Phi_{\alpha}^{[A]}\right\rangle\left|\Phi_{\alpha}^{[B]}\right\rangle,
$$

where $\chi_{A} \leq d$, i.e. the number of coefficients (Schmidt coefficients) of the expansion is bounded by the dimension of the smallest of the two subspaces of the partition. This simply indicates that the number of degrees of freedom that can be entangled between $A$ and $B$, is the maximum number of degrees of freedom of the smaller subsystem. If there is a single coefficient different from zero in the expansion, then 
the two parties are in a product state, i.e. not entangled. The coefficients of the expansion are unique (up to the degeneracy of the reduced density matrices), real and $\sum_{\alpha} \lambda_{\alpha}^{2}=1$. Notice also that $\rho_{A}=\operatorname{Tr}_{B}(|\Psi\rangle\langle\Psi|)=\sum_{\alpha} \lambda_{\alpha}^{2}\left|\Phi_{\alpha}^{[A]}\right\rangle\left\langle\Phi_{\alpha}^{[A]}\right|$ and $\rho_{B}=\operatorname{Tr}_{A}(|\Psi\rangle\langle\Psi|)=\sum_{\alpha} \lambda_{\alpha}^{2}\left|\Phi_{\alpha}^{[B]}\right\rangle\left\langle\Phi_{\alpha}^{[B]}\right|$. The Schmidt decomposition is nothing else than the usual Single Value Decomposition (SVD) of the coefficient matrix $C$ corresponding to the decomposition of $|\Psi\rangle$ in an arbitrary orthogonal basis $|i\rangle_{A}$ $\left(|j\rangle_{B}\right)$ :

$$
|\Psi\rangle=\sum_{i, j} C_{i, j}|i\rangle_{A}|j\rangle_{B}
$$

$C_{i, j}$ is a $d \times d^{M-1}$ matrix. Applying the Single Value Decomposition to $\mathrm{C}$ one obtains that $C=U D V$, where $U(V)$ is a unitary matrix of dimensions $d \times d$ $\left(d^{M-1} \times d^{M-1}\right)$ and $D$ is a diagonal matrix of dimensions $d \times d^{M-1}$. Therefore,

$$
\begin{aligned}
|\Psi\rangle & =\sum_{i, j} C_{i, j}|i\rangle_{A}|j\rangle_{B}=\sum_{\alpha}\left[\sum_{l} U_{l, \alpha}|i\rangle_{A}\right] D_{l, l}\left[\sum_{l} V_{\alpha, l}|j\rangle_{B}\right] \\
& =\sum_{\alpha=1}^{d} \lambda_{\alpha}\left|\phi_{\alpha}^{[A]}\right\rangle\left|\phi_{\alpha}^{[B]}\right\rangle .
\end{aligned}
$$

An iterative treatment of the above procedure (i.e. splitting now part $B$ into a block of 1 lattice site and block of $M-2$ sites and finding its Schmidt decomposition, and again for the block of $M-3$ sites) leads to the following expression:

$$
C_{i_{1} \ldots i_{M}}=\sum_{\alpha_{1} \ldots \alpha_{M-1}} \Gamma_{\alpha_{1}}^{[1] i_{1}} \lambda_{\alpha_{1}}^{[1]} \Gamma_{\alpha_{1}, \alpha_{2}}^{[2] i_{2}} \lambda_{\alpha_{2}}^{[2]} \Gamma_{\alpha_{2}, \alpha_{3}}^{[3] i_{3}} \ldots \Gamma_{\alpha_{M-1}}^{[M] i_{M}} .
$$

The tensors $\Gamma$ take into account the correlations arising from the splitting, and are straightforwardly obtained by performing at each bipartite partition the corresponding SVD. Notice that except for the first and the last lattice site, for all the other lattice sites, the corresponding tensor $\Gamma$ depends on two $\alpha$ indices, corresponding to the two involved partitions, and takes into account the correlations with the block at its left, and the block at its right. It can be shown that for translationally invariant $1 \mathrm{D}$ systems with short range interactions, the coefficients of the Schmidt decomposition behave as $\lambda_{\alpha}^{[l]} \sim \exp (-\alpha)$, and the truncation of the Hilbert space is performed by removing all small coefficients.

By grouping the $\Gamma$ tensors with the coefficients $\lambda$ the expression of the ansatz takes the more compact form:

$$
|\Psi\rangle=\sum_{i_{1}, \ldots i_{M}=0}^{d} \operatorname{Tr}\left[A^{[1], i_{1}} \ldots A^{[M], i_{M}}\right]\left|i_{1}, \ldots i_{M}\right\rangle,
$$

where $A^{[k], 1} \ldots A^{[k], d}$ are $D_{k} \times \tilde{D}_{k}$ complex matrices with $D_{k+1}=\tilde{D}_{k} \leq D$, and $d$ is the occupation number on site $i$. Operations on a single site $i$ involve only updating the value of $A^{[k], i_{k}}$, which basically implies of the order of $D^{2}$ operations, $A^{\prime[k], i_{k}}=U A^{[k], i_{k}}$. In a similar way, operations involving two neighbouring sites correspond to updating the matrices of the corresponding sites and the correlations between them and again can be efficiently performed. Thus, the ground state of 

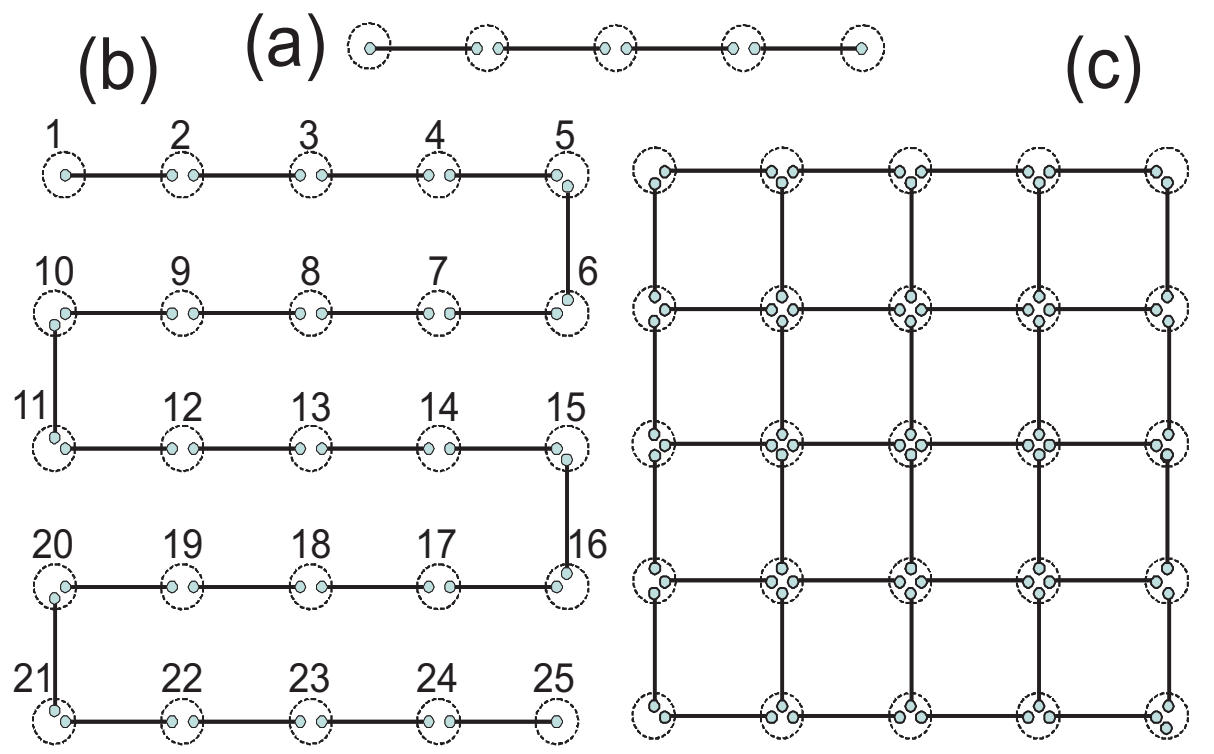

Figure 5. Schematic representation of a) MPS for a 1D system, b) "inefficient" MPS in 2D, and c) PEPS for a 2D system. Each pair of nearest neighbour sites is connected via maximally entangled state from an auxiliary Hilbert space. In a) and b) matrices, $A$ projects onto the physical space. In c) matrices have to be replaced by 4 th rank tensors in the bulk (from [306]).

the Bose-Hubbard Hamiltonian, which includes only next neighbour interactions plus on site collisions can be calculated variationally, whereas time evolutions can be simulated by means of a Trotter expansion. (For the recent work of dynamical response of $\mathrm{BH}$ model at the MI-SF transition, see [305].)

3.10.2 Matrix product states. Already Östlund and Rommer [307,308] realized that DMRG leads to an special type of ansatz known as Matrix Product States [309,310]. Matrix Product States (MPS) and their generalization to higher dimensions, Projected entangled-pair states (PEPS) (see Fig. 5) corresponds to translationally invariant states, whose general expression is of the form [308] :

$$
|\Psi\rangle=\sum_{i_{1}, \ldots i_{M}=1}^{d} \operatorname{Tr}\left[A^{[1], i_{1}} \ldots A^{[M], i_{M}}\right]\left|i_{1}, \ldots i_{M}\right\rangle,
$$

which describes a chain of $M$ spins of dimension $d$. The $A^{[k] i_{k}}$ are complex matrices with dimension $\leq D \times D$ matrices. The reason why this type of states (MPS) can reproduce very well the ground states of quantum spin systems with nearest neighbor interactions, is because the ground states (that have also the translational invariance of the Hamiltonian) and low energy excitations are completely determined by their two-body reduced density operators as shown by Verstraete and Cirac [311]. An intuitive but handwaving argument to understand the reason why MPS parametrize ground states of gapped quantum spin systems, comes from Hastings theorem [312], which states that for gapped systems all correlations de- 
cay exponentially. This means that since $\left\langle O_{A} O_{B}\right\rangle-\left\langle O_{A}\right\rangle\left\langle O_{B}\right\rangle \simeq \exp \left(-l_{A B} / \xi_{\text {corr }}\right)$, blocks $A$ and $B$, separated by $l_{A B}$, can be described by a product state, when $l_{A B}$ is much larger than the correlation length.

MPS and PEPS have already proven to be an enormously useful tool. In particular, they can be easily extended to calculate time evolution. They have also been used to characterize quantum phase transitions in spin chains [300], simulate infinite systems [313], and establish a closer relation between criticality, area law, and block entanglement [314].

\subsection{Fermi and Fermi-Bose Hubbard models}

Most of the methods that are used for Bose-Hubbard models (some of which were discussed in the previous subsections of this section) can be carried over to fermionic systems, although typically, treatment of fermions is more difficult. For example, mean field BCS method, valid for weakly interacting fermions, requires much more effort for the trapped gases than the bosonic Bogoliubov-de Gennes approach. Quantum Monte Carlo methods (QMC) suffer notoriously the, so-called, "sign problem" (negative probabilities). Gutzwiller ansatz in dimensions higher than 1, does not take the fermionic anticommutation relations into account properly, due to non-existence of standard Jordan-Wigner transformation (for a discussion, see for instance [183]).

When we deal with Fermi-Fermi, or Fermi-Bose mixtures, it is always useful to study some limiting cases, that moreover, can be realized in experiments. One limit would be the weakly interacting regime, where Hartree-Fock, BCS or Landau Fermi liquid theory could be applied. In the strong coupling limit of Mott insulating states, a very useful method is to construct an effective Hamiltonian. Such Hamiltonian will often correspond to a spin model, if we deal with commensurate filling factors (as we discussed in the Introduction). If the Mott states involve states differing by odd number of fermions (for instance presence or absence of a fermion), then the effective model will necessarily involve composite fermions.

Fermionic Hubbard models. Perhaps the most well-known are the effective models obtained in the strong coupling limit of the electronic Hubbard model for spin $1 / 2$ fermions, described by the Hamiltonian,

$$
H=-t \sum_{\langle i, j\rangle, \sigma}\left(f_{i \sigma}^{\dagger} f_{j \sigma}+\text { h.c. }\right)+\frac{U}{2} \sum_{i} f_{i \uparrow}^{\dagger} f_{i \downarrow}^{\dagger} f_{i \downarrow} f_{i \uparrow}-\mu \sum_{i, \sigma} f_{i \sigma}^{\dagger} f_{i \sigma} .
$$

In the limit of large repulsive interactions, $U \gg t, \mu$, there can be one, or zero particles at a site. In this limit the model is termed in the literature " $t-J$ " model (cf. [60]). When the system is at full filling, i.e. there is 1 fermion at each site (in the Mott limit), the effective Hamiltonian reduces to that of Heisenberg quantum antiferromagnet with the magnetization fixed by the corresponding numbers of spin-up and spin-down fermions (or external "magnetic" field). In particular, if these numbers are equal, the system has a zero net magnetization (vanishing "magnetic" field). Allowing for certain number of unoccupied sites, is termed doping. Doped Mott insulators provide a key to understand high $T_{c}$ superconductivity of cuprates (for a recent review, see [198]; for the recent contribution in the 
atomic context, see [199]). P. Torma's group has investigated in recent years, the role of spin-density imbalance, both in trapped [200], and superfluid lattice Fermi gases [201, 202]. Asymmetric Hubbard model with light and heavy fermions has been studied by Ziegler's group, who has, in particular, shown quantum phases with self-induced disorder [203-205].

Hubbard models for particles with higher spin, as well as Fermi-Fermi mixtures can be realized with ultracold atoms. There has been thus considerable interest in studying such systems (for a review of $S U(N)$ symmetric Hubbard models of fermions and bosons see [206]). In Ref. [122] effective spin Hamiltonians for spin $3 / 2$ ultracold spinor gases, described by the spinor version of the Hamiltonian (54) have been derived and investigated. In 1D the same system has been studied using the bosonisation approach [207], and exact Bethe ansatz method [208].

Fermi-Bose Hubbard models. The spectacular advances in loading atomic samples in optical lattices have allowed for the realization of systems which are well described by a Fermi Hubbard model, as well as mixtures of fermions and bosons (FB) described by Fermi-Bose Hubbard models. In the latter case, and in the limit of strong atom-atom interactions, such systems can be described in terms of composite fermions, consisting of a bare fermion, or a fermion paired with one boson (bosonic hole), or two bosons (bosonic holes), etc. [209]. The quantum phase diagram displayed by those systems is amazingly rich and complex. The physics of Fermi-Bose mixtures in this regime has been studied recently in [167, 183, 210], using perturbation theory up to second order to derive an effective Hamiltonian. There are a number of recent studies of FB mixtures in optical lattices ${ }^{1}$, and also of strongly correlated FB mixtures in traps [166,218]. In particular, the validity of the effective Hamiltonian for fermionic composites in 1D was studied using exact diagonalization and Density Matrix Renormalization Group method in Ref. [219], whereas trap effects were analyzed in Ref. [220]. Very recently, exact Bethe ansatz solution for the special case of FB Hubbard model has been found and intensively studied $[221,222]$. Also, intensive studies of quantum phases in the multiband FermiHubbard model (that models a Fermi gas subject to Feshbach resonance) have been undertaken [223-225]. Recently, particular interest has been stimulated by the experiments of the Hamburg and ETH groups [226,227], in which presence of fermions attracting bosons was seen to decrease the lattice potential threshold for MI state. This is quite paradoxical, since DMRG calculations predict the opposite effect [228] The physics of disordered FB mixtures will be reported in subsection 4.7.

Here, we focus on the homogeneous case and explain how to derive the corresponding effective Hamiltonian. We consider a homogeneous mixture of ultracold bosons (b) and spinless (or spin-polarized) fermions (f), for example ${ }^{7} \mathrm{Li}^{6}{ }^{6} \mathrm{Li}$ or ${ }^{87} \mathrm{Rb}-{ }^{40} \mathrm{~K}$, trapped in an optical lattice. As for the bosonic case, in the tightbinding regime, it is convenient to project the wave functions onto the Wannier basis of the fundamental Bloch band, corresponding to wave functions well localized in each lattice site $[155,229]$. This leads to the Fermi-Bose Hubbard (FBH) Hamiltonian [29,60, 154, 218]:

\footnotetext{
${ }^{1}$ The studies of trapped FB gases concerned in particular FB phase separation $[211,212]$, the phase diagram [213], novel types of collective modes [212,214], Fermi-Fermi interactions mediated by bosons $[212,215]$, the collapse of the Fermi cloud in the presence of attractive FB interactions [216], or the effects characteristic for the 1D FB mixtures [217].
} 
$H_{F B H}=-\sum_{\langle i j\rangle}\left[t_{b} b_{i}^{\dagger} b_{j}+t_{f} f_{i}^{\dagger} f_{j}+h . c.\right]+\sum_{i}\left[\frac{U}{2} n_{i}\left(n_{i}-1\right)+V n_{i} m_{i}-\mu^{b} n_{i}-\mu^{f} m_{i}\right]$,

where $b_{i}^{\dagger}, b_{i}, f_{i}^{\dagger}$ and $f_{i}$ are bosonic and fermionic creation- annihilation operators of a particle in the $i$-th localized Wannier state of the fundamental band, and $n_{i}=$ $b_{i}^{\dagger} b_{i}, m_{i}=f_{i}^{\dagger} f_{i}$ are the corresponding on-site number operators. The FBH model describes: (i) nearest neighbor (n.n.) boson (fermion) hopping, with an associated negative energy, $-t_{b}\left(-t_{f}\right)$; (ii) on-site boson-boson interactions with an energy $U$, which we will assume to be repulsive (i.e. positive),(iii) on-site boson-fermion interactions with an energy $V$, which is positive (negative) for repulsive (attractive) interactions and finally (iv) on-site energy due to chemical potentials in grand canonical description. Following $[87,183,210,230]$, let us review how to derive an effective Hamiltonian to second (or higher) order in $t=t_{f}=t_{b}$ (we assume here the same tunneling for bosons and fermions for the sake of simplicity). Generalization to the case $t_{f} \neq t_{b}$ is straightforward. We follow here the notation of Ref. [167].

In the limit of vanishing tunneling $(t=0)$ with finite repulsive boson-boson interaction $U$, and in the absence of interactions between bosons and fermions $(V=0)$, the bosons are in a Mott insulator (MI) phase with exactly $\tilde{n}=\left\lceil\tilde{\mu}^{b}\right\rceil+1$ bosons per site, where $\tilde{\mu}^{b}=\mu^{b} / U$ and $\lceil x\rceil$ denotes the integer part of $x$. In contrast, the fermions can be in any set of Wannier states, since for vanishing tunneling, the energy is independent of their configuration. The situation changes when the interparticle interactions between bosons and fermions, $V$, are turned on. In the following, we define $\alpha=V / U$, and consider the case of bosonic MI phase with $\tilde{n}$ bosons per site. We also assume that the temperature is small enough $(T<V)$ in order to neglect the particle-hole excitations. It follows that tunneling of a fermion is necessarily accompanied by the tunneling of $-s$ bosons (if $s<0$ ) or opposed-tunneling of $s$ bosons (if $s \geq 0$ ). The dynamics of the Fermi-Bose mixture can thus be regarded as the one of composite fermions made of one fermion plus $-s$ bosons (if $s<0$ ) or one fermion plus $s$ bosonic holes (if $s \geq 0$ ). The annihilation operators of the composite fermions are [167]:

$$
\begin{aligned}
& F_{i}=\sqrt{\frac{(\tilde{n}-s) !}{\tilde{n} !}}\left(b_{i}^{\dagger}\right)^{s} f_{i} \text { for } s \text { bosonic holes } \\
& F_{i}=\sqrt{\frac{\tilde{n} !}{(\tilde{n}-s) !}}\left(b_{i}\right)^{-s} f_{i} \text { for }-s \text { bosons. }
\end{aligned}
$$

These operators are fermionic in the sub-Hilbert space generated by $|n-m s, m\rangle$ with $m=0,1$ in each lattice site. Note that within the picture of fermionic composites, the vacuum state corresponds to MI phase with $\tilde{n}$ boson per site. At this point, different composite fermions appear, depending on the values of $\alpha, \tilde{n}$ and $\tilde{\mu}^{b}$, as detailed in Fig. 6 .

Because all sites are equivalent for the fermions, the ground state is highly $\left[N ! / N_{f} !\left(N-N_{f}\right) !\right]$-degenerated, where $N$ denotes the total number of atoms and $N_{f}$ the number of fermions. Hence, the manifold of ground states is strongly coupled by fermion or boson tunneling. We assume now that the tunneling rate $t$ is 


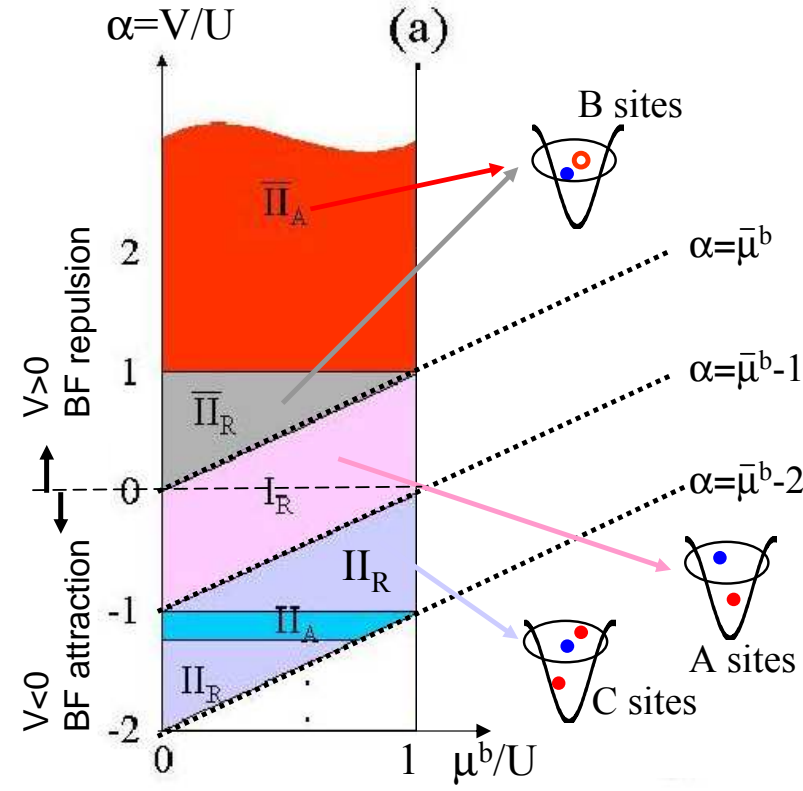

(b)

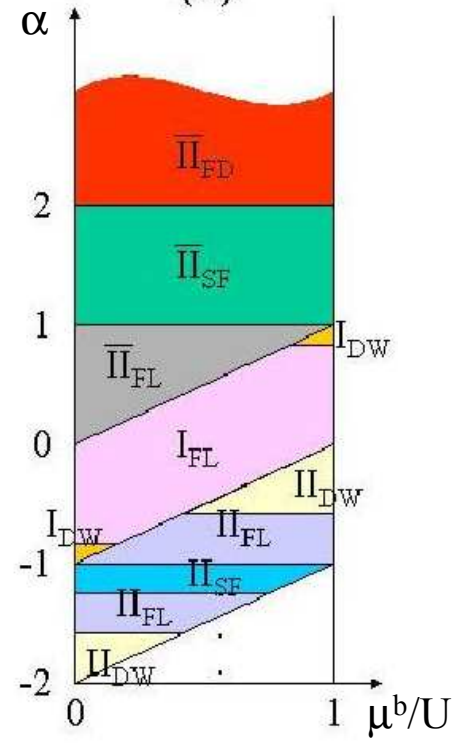

Figure 6. Quantum phase diagrams of Fermi-Bose mixtures in an homogeneous optical lattice as functions of $\tilde{\mu}^{b}$ and $\alpha=V / U$, for $\rho_{f}=0.4$ and $t / U=0.02$. Roman numbers denote the total number of particles that form the composite and a bar means that the composite is formed by bosonic holes rather than bosons. (a) Diagram of composites where the filled small (blue) dots symbolize fermions, large (red) dots symbolize bosons and empty (red) dots, bosonic holes. The subindex A (R) indicates attractive (repulsive) composites interactions. (b) Detailed quantum phase diagram of fermionic composites. The subindices denote here different phases: DW (density wave), FL (fermi liquid), SF (superfluid) and FD (fermionic domains). The strongly correlated phases for small but finite $t$ are surrounded by characteristic lobes [183], beyond which bosons become superfluid. Therefore, there are thin regions of bosonic superfluid between the various composite phases (from [167]).

small but finite. In the homogeneous case, one uses standard second order perturbation theory, to derive an effective Hamiltonian [60] for the fermionic composites (for the disordered case, see Appendix A $[87,230]$ ):

$$
H_{e f f}=-d_{e f f} \sum_{\langle i, j\rangle}\left(F_{i}^{\dagger} F_{j}+\text { h.c. }\right)+K_{e f f} \sum_{\langle i, j\rangle} M_{i} M_{j}-\bar{\mu}_{e f f} \sum_{i} M_{i},
$$

where $M_{i}=F_{i}^{\dagger} F_{i}$ and $\bar{\mu}_{\text {eff }}$ is the chemical potential, which value is fixed by the total number of composite fermions. The nearest neighbor hopping for the composites is described by $-d_{e f f}$ and the nearest neighbor composite-composite interactions is given by $K_{e f f}$, which may be repulsive $(>0)$ or attractive $(<0)$. This 
effective model is equivalent to that of spinless interacting fermions. The interaction coefficient $K_{\text {eff }}$ originates from 2 nd order terms in perturbative theory and can be written in the general form [183]:

$K_{e f f}=\frac{-2 t^{2}}{U}\left[(2 \tilde{n}-s)(\tilde{n}+1)-s(\tilde{n}-s)-\frac{(\tilde{n}-s)(\tilde{n}+1)}{1+s-\alpha}-\frac{(\tilde{n}-s+1) \tilde{n}}{1-s+\alpha}-\frac{1}{s \alpha}\right]$.

This expression is valid in all the cases, but when $s=0$, the last term $(1 / s \alpha)$ should not be taken into account. $d_{\text {eff }}$ originates from $(|s|+1)$-th order terms in perturbative theory and thus presents different forms in different regions of the phase diagram of Fig. 6. For instance in region $\mathrm{I}, d_{\text {eff }}=t$, in region $\overline{I I} d_{\text {eff }}=2 t^{2} / \mathrm{V}$ and in region II, $d_{e f f}=4 t^{2} /|V|$.

The physics of the system is determined by the ratio $K_{\text {eff }} / d_{e f f}$ and the sign of $K_{\text {eff }}$. In Fig. 6(a), the subindex A/R denotes attractive $\left(K_{\text {eff }}>0\right) /$ repulsive $\left(K_{\text {eff }}<0\right)$ composites interactions. Fig. 6(b) shows the quantum phase diagram of composites for fermionic filling factor $\rho_{f}=0.4$ and tunneling $t / U=0.02$. For large values of $t / U$, a transition to the $\mathrm{SF}$ state takes place (for the corresponding $3 \mathrm{D}$ lobes over the different regions in Fig. 6, see Ref. [183]).

\section{Disordered ultracold atomic gases}

\subsection{Introduction}

In 1958, Anderson [66] reported for first time the quantum localization phenomenon. He pointed out that the extended wave functions of electrons (Bloch waves) in a crystal, for strong enough disorder, become localized with an exponentially decaying envelope $|\psi| \sim \exp \left(\left|\vec{r}-\overrightarrow{r_{i}}\right| / \xi\right)$, where $\xi$ is the localization length. This occurs at the single particle level by coherent back scattering from random impurities. In one dimension, it has been rigorously proven that infinitesimally small disorder leads to exponential localization of all the eigenfunctions by repeated backscattering $[67,315,316]$ and it is known that the localization length is of the order of the backscattering mean free path. Ref. [317] proposed the concept of mobility edge, which separates the localized states from the extended ones. The scaling theory has been one of the basic tools to deepen the understanding of the Anderson localization phenomenon by considering the conductance as a function of the system size or of other scale variables [318-323]. In $1 \mathrm{D}$ the localization length is a function of the ratio between the potential and the kinetic (tunneling) energies of the eigenstate and the disorder strength. For the case of discrete systems with constant tunneling rates and local disorder distributed according to a Lorentzian distribution (Lloyd's model, cf. [324]) the exact expression for the localization length is known. In general, an exact relation between the density of states and the range of localization in $1 \mathrm{D}$ has been provided by Thouless [319]. In 2D, following the scaling theory, it is believed that localization occurs also for arbitrarily small disorder, but its character interpolates smoothly between algebraic for weak disorder, and exponential for strong disorder. There are, however, no rigorous arguments to support this belief, and several controversies aroused about this subject over the years. In 3D, scaling theory predicts a critical value of disorder, above which every 
eigenfunction exponentially localizes, and this fact has found strong evidence in numerical simulations.

Since the discovery of Anderson [66], the field of quantum disordered systems has been a very active research field in condensed matter physics (for reviews see [325$328]$ ). Disordered systems appear in some of the most challenging open questions concerning many body systems (see for instance [329-331]). In particular, quenched (i.e., frozen on the typical time scale of the considered systems) disorder determines the physics of a large variety of phenomena: transport, conductivity, localization effects and metal-insulator transition (cf. [325-328]), spin glasses (cf. [83,154,332]), neural networks (cf. [333]), percolation [334,335], high $T_{c}$ superconductivity (cf. [60]), or quantum chaos (cf. [324]).

The theoretical studies of disordered systems imply severe difficulties. One of the main ones is that in order to characterize the systems independently of the particular disorder realisations, one needs to average over the disorder. Such quenched (frozen over the typical time scales of the system) average of physical quantities, such as for instance free energy, requires usually the use of special methods, such as the replica trick (cf. [83]) or supersymmetry method [336]. Interestingly, quantum information approach might help to calculate averages using quantum superpositions, see [337]. Regarding numerical approaches, the characterization of the system demands either simulations of very large samples to achieve "self-averaging" or numerous repetitions of simulations of small samples with different configurations of the disorder. Obviously, this difficulty is particularly important for quantum disordered systems. Another difficulty that arises in the study of disordered systems is the possible existence of a large number of low energy excitations. Similar limitations arise in frustrated systems (cf. [107])(see section 5). Last but not least, the interplay of the disorder and the interactions has been and still is a challenging problem, as it is reported in the following subsections in the case of bosons and fermions.

Disorder effects are present in many condensed matter systems, and in particular in electronic systems. Nevertheless, these systems do not provide the best possible scenario to test the theoretical predictions mainly because of two factors: (i) the disorder is not controllable, but fixed by the specific realization of the sample; and (ii) the electrons interact via the long-range Coulomb interaction, which is fixed by Nature. It is thus desirable to ask whether atomic, molecular physics and quantum optics may help to understand quantum disordered systems, for instance by realizing in experiments disordered models using cold atoms in optical lattices. The advantages of atomic disordered systems are clear: (i) the control of the generation of random potentials that induce disorder in the system (see subsections 4.3 and 4.5); (ii) the presence of interactions that can also be controlled by means of Feshbach resonances; (iii) the type of quantum statistics, that can be chosen by using ultracold bosons or fermions, and (iv) the full control of the trapping potentials and therefore the effective dimensionality of the system. Obviously, standard control parameters, such as for example the temperature of the system, can also be controlled, but in these aspects atomic system do not differ much form the condensed matter one. Of course, there are also disadvantages: atomic systems are "small". Typical experiments with ultracold lattice gases involve lattices of sizes up to $100 \times 100 \times 100$ and up to few times $N=10^{6}$ atoms. 


\subsection{Disordered interacting bosonic lattice models in condensed matter}

The theory of disordered interacting bosons is complex and there are essentially no exact solutions, not even in one-dimension. In this section we review different numerical and approximate results existing in the condensed matter literature.

A system of bosons in a lattice with short-ranged repulsive interactions in the presence of external random potentials was considered in [30] where, by treating the tunneling as a perturbation, the phase diagram of the system was worked out. The three possible ground states predicted were: (i) an incompressible Mott insulator with a gap for particle-hole excitations; (ii) a gapless Bose-glass insulator with finite compressibility, exponentially decaying superfluid correlations in space and infinite superfluid susceptibility; and (iii) a superfluid phase with the usual off-diagonal long ranged order. It was predicted that the gapless Bose-glass intervenes between the Mott and the superfluid phases, so that the superfluid transition always occurs from the Bose-glass. The critical properties of this transition were characterized by three exponents: a dynamical exponent that equals the dimensionality of the system, a correlation length exponent that is bounded from above and an order parameter exponent that is bounded from below.

Previously, the onset of superfluidity in a random potential was studied in [338] and [339]. In [338], a system of strongly disordered hard-core bosons was considered in the framework of a mean field theory including quantum fluctuations. A renormalization group approach was developed to study a one-dimensional system of interacting bosons in a random potential [339]. In this work it was shown the existence of a localized-superfluid transition and universal power laws for correlation functions on the transition line were found. The case of zero temperature interacting bosons at commensurate density (one atom per site) on disordered lattices in one and two dimensions was addressed in [340] by using a real-space renormalization group. The results showed that when weak disorder is introduced, a transition directly from the Mott insulator to the superfluid occurs, so that infinitesimally weak disorder does not stabilize a Bose-glass at commensurate filling. The Boseglass is found beyond a threshold disorder, in contradiction with the arguments in [30]. The critical exponents for the superfluid-Bose-glass phase transitions at zero temperature for hard-core bosons in one-, two-dimensional [341] and threedimensional [342] disordered lattices were calculated using a quantum real-space renormalization group method once the system was mapped onto a quantum spin1/2 XY model with transverse random field. From these calculations [341,342], it was concluded that randomness is always relevant in one-dimensional systems while in two and three dimensions, there is a critical amount of disorder below which the superfluid phase is stable. Moreover it is also stated that there is only one universality class for the superfluid-Bose-glass transition. Starting from the mapping of a system of hard core disordered bosons onto a quantum spin- $1 / 2 \mathrm{XY}$ model with transverse random field and generalizing it to a system of spins with arbitrary magnitude, a perturbative study was applied to get more insight into the low-energy excitations in a weakly disordered bosonic system [343].

In the early 90's, disordered Bose condensates were studied using the Bogoliubov approximation [344-346]. In [344], the screening of the random potential in a twodimensional dense Bose gas in a lattice due to the short-range repulsive interactions was addressed. This screening is effective when the healing length is short enough so that the condensate can adjust to the variations of the random potential. The 
effects of the fluctuations deplete the condensate nonuniformly and lead to a spectrum of collective, phonon-like excitations. In [346] was reported that weak disorder hardly affects the condensate fraction or the superfluid density although the condensate distorts to screen the imposed random potential. In the strong disorder limit, the condensate fraction and the superfluid density tend to vanish as the disorder increases and a constant density of states appears at low energy. In case of three-dimensional bosonic systems in random external potentials the depletion of the condensate and the superfluid density were explored in [345]. The conclusion obtained from this analysis was that disorder is more active in reducing superfluidity than in depleting the condensate. In this scenario, a formalism based on dispersive quantum hydrodynamics at zero temperature was applied to investigate the propagation of phonons in the system [347]. In particular, the shift of the sound velocity and its damping were calculated. In [348], building on the continuum model of [345] and [347], a systematic diagrammatic perturbation theory for a dilute Bose gas with weak disorder at finite temperature below the superfluid transition temperature was developed and the disorder-induced shift of the superfluid transition temperature was derived.

Monte Carlo techniques (world-line algorithm) have been also applied to study the interplay between interactions and disorder in bosonic systems. In [262] was reported the first convincing evidence of a second insulating phase, the Andersonglass phase, in a one-dimensional lattice system. The Anderson-glass phase appears at weak couplings where the interactions compete with the disorder and tends to delocalize the bosons, contrary of what happens in the Bose-glass phase, that appears at strong couplings where disorder and interactions cooperate. Moreover, these two phases differ substantially on the nature of the boson density distribution: in the Bose-glass, the density is reasonably uniform while in the Anderson-glass, boson density correlations are expected to decay exponentially [262]. The existence of two such separate insulating phases was conjectured previously in [339]. The same year 1991, in [349], path-integral Monte Carlo techniques were used to study also the superfluid-insulator transition but in a two-dimensional square lattice. Using this technique in two dimensions, three phases were predicted: superfluid, Bose-glass and Mott-insulator and it was stated that at commensurate density, the system seems to undergo a direct transition from the superfluid phase to Mott insulator contradicting the picture of disorder of [30] and [339], but in agreement with the results obtained using real-space renormalization group by [340]. The direct transition from superfluid to Mott insulator without intervening Bose-glass at weak disorder was also reported for instance in [186] by using density-matrix renormalization group in one dimension, in [350] using quantum Monte Carlo simulations in two dimensions, or in [351] using a mean field theory. Contrarily, the transition via the Bose-glass phase was predicted, for instance in $[352,353]$ by using Monte Carlo simulations, in [354] by using the renormalization group method, in [193] using a strong-coupling expansion for the phase boundary of the Mott-insulator or in [355] by an improved application of the density matrix renormalization group with respect to [186]. It is worth noticing that in the results of [355] there is no indication of a qualitative difference between the glass phase at small or large values of the repulsion i.e., between Anderson and Bose-glass. Thus, a complete understanding of the phase diagram of interacting bosons in the presence of disorder and in various dimensions is still under debate. In particular, the possibility of a direct Mott-insulator to superfluid transition in the presence of disorder remains a 
controversial issue.

\subsection{Realization of disorder in ultracold atomic gases}

In order to perform a detailed analysis of the properties of the disordered interacting Bose lattice gases it would obviously be very useful to have an experimentally accessible system, that could be studied in a controlled way. As we will see below, ultracold atomic lattice gases provide such opportunity.

As has been discussed in section 1, since the experimental realization of BoseEinstein condensation there has been an enormous progress in the studies of the ultracold gases: first in the weakly interacting regime and more recently also in the strongly interacting regime. Nowadays there exist a complete control of the generation and manipulation of ultracold bosonic, or fermionic gases, as well as their mixtures. Among all the techniques of control, the transfer of these ultracold samples to optical lattices offers an unprecedented possibility to study disorder related phenomena. In fact, by superimposing laser beams from different directions and with different frequencies, it is possible to generate a huge variety of lattice geometries in a very controlled way. For instance, it has been proposed the use of two colour superlattices [68-70], i.e., the superposition of two standing-wave lattices with comparable amplitudes and with different wavelengths, as a form of quasidisorder. The so-called quasicrystal optical lattices in two and three dimensions have also been explored in [356-359]. These systems present long range order but not translational invariance. An example of a laser configuration [359] that gives rise to a quasicrystal lattice consists on $N_{b}$ laser beams arranged on the $x y$ plane with $N_{b}$-fold symmetry rotation. The polarization $\vec{\epsilon}_{j}$ of laser $j$ with wavevector $\vec{k}_{j}$ is linear and makes an angle $\alpha_{j}$ with the $x y$ plane. The optical potential is in this case:

$$
V(\vec{r})=\frac{V_{0}}{\left|\sum_{j} \varepsilon_{j}\right|^{2}}\left|\sum_{j=0}^{N_{b}-1} \varepsilon_{j} \vec{\epsilon}_{j} \exp ^{-i\left(\vec{k}_{j} \vec{r}+\varphi_{j}\right)}\right|^{2},
$$

where $\vec{r}=[x, y], 0 \leq \varepsilon_{j} \leq 1$ stand for eventually different laser intensities and $\varphi_{j}$ are the corresponding phases. In [68] a two-dimensional local quasidisordered potential was numerically simulated by using a main optical lattice, $V_{l}(\vec{r})$, to which a secondary lattice, $V_{r}(\vec{r})$, with much smaller amplitude and with frequency incommensurable with respect to the main lattice, is superimposed. The quasidisorder potential in this case reads:

$$
V(\vec{r})=V_{l}(\vec{r})+V_{r}(\vec{r})=V_{0}\left[\cos ^{2}(k x)+\cos ^{2}(k y)\right]+V_{1}\left[\cos ^{2}\left(\overrightarrow{k_{1}} \vec{r}\right)+\cos ^{2}\left(\overrightarrow{k_{2}} \vec{r}\right)\right]
$$

where $\vec{r}=[x, y]$. The secondary lattice is responsible for the introduction of the (quenched) pseudodisorder which is determined by the ratio between the wavelengths of the main and additional lattices, $k_{1} / k$ and $k_{2} / k$. The same kind of potential has been also studied in one-dimensional geometries [360].

All the potentials discussed so far in this section are not strictly speaking disordered, but quasidisordered. Truly random potentials can be achieved by using a speckle pattern $[68,71-74,361-363]$. The speckle field is a light field with highly disordered intensity and phase distributions but stationary and coherent. Such a speckle field 


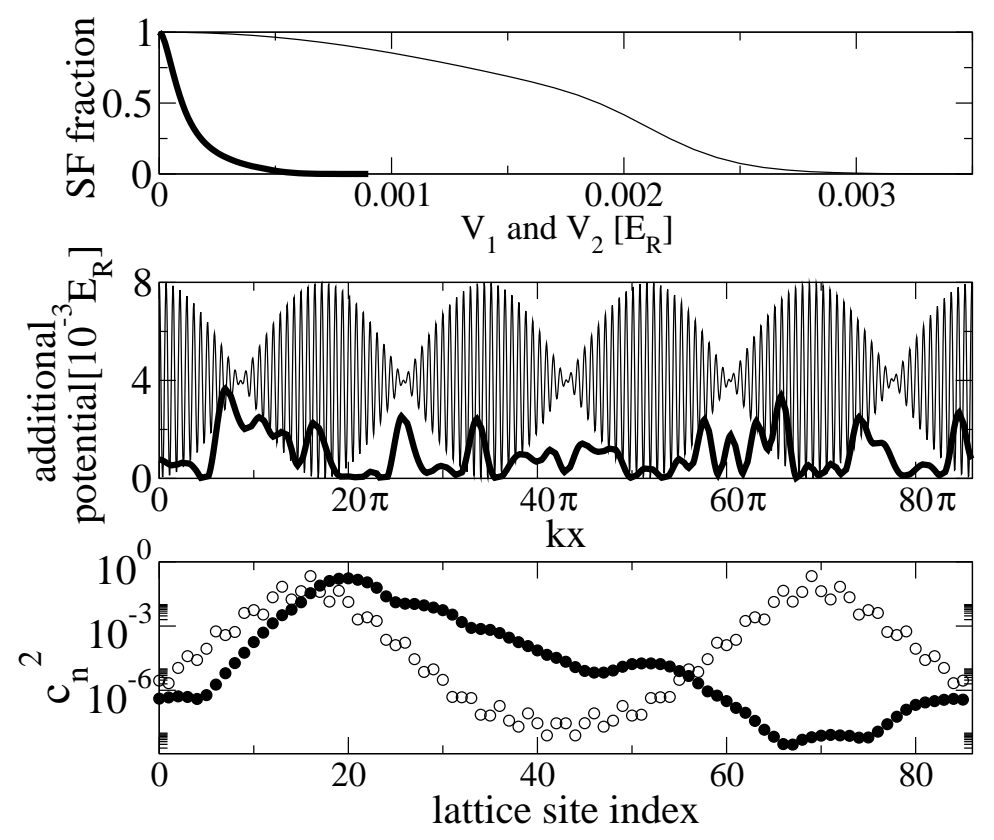

Figure 7. Theoretical prediction of Bose-Anderson glass in a 1D optical lattice; top: superfluid fraction as a function of disorder $V_{1}$ (thickline), respectively quasi-disorder $V_{2}$ (thinline); middle: disordered speckle potential (thickline), and quasi-disordered (thinline) potential formed by incommensurable superlattices; bottom: occupation numbers of the ground state in the presence of disorder (black dots), and quasi-disorder (open dots) (from [68]).

can be easily generated experimentally, for instance, by introducing a diffusor in the path of a laser plane wave or by reflecting a laser beam in a surface that is rough on the scale of the laser wavelength. Specifically, the random intensity of an speckle field follows an exponential statistical distribution with a standard deviation given by the average intensity, $P(I)=\exp (-I /\langle I\rangle)$. In addition, the intensity correlation length or disorder correlation length, given by the half-width of the autocorrelation function, is limited by optical resolution being of the order of at least few $\mu \mathrm{m}$ (see Fig. 7 for examples of 1D random, and quasi-random potentials leading to Bose-Anderson glass).

One can also create a disordered potential by using atoms of a second species or internal state quenched randomly at the nodes of an optical lattice $[364,365]$. These randomly distributed atoms act as point-like scatterers for the atoms of the first species, that are not trapped by the optical lattice. One possibility to randomly trap the atoms of the second species is by rapidly quenching them from the superfluid to the Mott insulating phase. In this approach, the correlation length is very short since the distance between the lattice sites is of the order of $0.5 \mu \mathrm{m}$. It is possible that such quenched random scatterers of fermionic composites (consisting of fermions bounded to 6-7 bosons) have been recently realised in an experiment with FermiBose mixtures by the Hamburg group [36]. It is also worth pointing out that with this kind of disorder, the effective Hamiltonian for composites will involve disorder with bimodal, or at most trimodal distribution. This kind of disorder is particularly 
interesting to study spin glass transition in the 2D Ising model [366].

Recently, also the generation of disorder in ultracold atoms in optical lattices through on-site interparticle interactions has been addressed [367,368]. The idea is to exploit the significant modifications of the scattering properties of two atoms that can be induced near Feshbach resonances [93,94] by slight modifications of the magnetic field. In this scenario, spatial random variations of the local interatomic interactions arise. The system is placed at the verge of a Feshbach resonance by means of an offset magnetic field in the presence of a spatially random magnetic field. This spatially random magnetic field appears for instance in magnetic microtraps and atom chips as a result of the roughness of the underlying surface [369]. The intrinsic disorder that appears in magnetic microtraps and atom chips has also been addressed in the absence of an optical lattice [370,371]. Controllable disorder on the level of next-neighbors interactions can be generated by means of tunneling induced interactions in systems with local disorder [86]. It is also worth mentioning a very recent attempt to create controlled disorder using optical tweezers methods [372].

\subsection{Disordered ultracold atomic Bose gases in optical lattices}

Previous section 4.3 reports on experimental feasible realizations of random and pseudorandom potentials for cold atoms in optical lattices. All these possibilities lead to experimental realizations of the disordered Bose-Hubbard model [68-70], where the interplay between interactions and disorder could be explored. The BoseHubbard hamiltonian in the presence of disorder reads like Eq. (12) but with site dependent tunneling rate, $t_{i j}$, and local chemical potential, $\mu_{i}$ :

$$
H=-\sum_{<i j>}\left[t_{i j} b_{i}^{\dagger} b_{j}+h . c .\right]+\sum_{i} \frac{U}{2} n_{i}\left(n_{i}-1\right)+\sum_{i} \mu_{i} n_{i},
$$

Although tunneling coefficients in the most general disordered case should be site dependent, it has been shown [68] that in optical lattices the hopping disorder is suppressed against on-site disorder included in the term $\mu_{i}$. The last term in (62) gives the on-site single-particle energy which originates from the external potentials and the on-site part of the kinetic energy. Therefore it accounts for an external harmonic trapping potential plus the inhomogeneities produced by the speckle pattern, or by the superlattice. Eventually, $\mu_{i}$ also contains the chemical potential in the grand canonical description. In [68], the pseudorandom on-site energies are calculated by using:

$$
\mu_{i}=\int d^{3} r w^{*}\left(\vec{r}-\overrightarrow{r_{i}}\right) V_{r}(\vec{r}) w\left(\vec{r}-\overrightarrow{r_{i}}\right)
$$

where $w\left(\vec{r}-\overrightarrow{r_{i}}\right)$ are the Wannier functions in the lowest Bloch band and $V_{r}(\vec{r})$ is the superlattice potential introduced in (61) or the potential induced by a numerically generated speckle pattern characterized by its mean value and the average speckle size. In $[69,70]$ there was considered a sinusoidal variation of the $\mu_{i}$ in the range $[-\Delta, 0]$ recovering the regular case for $\Delta=0$. In [68], the dynamical generation of the Bose-glass (strong interactions and non-integer filling factor) and the Anderson 


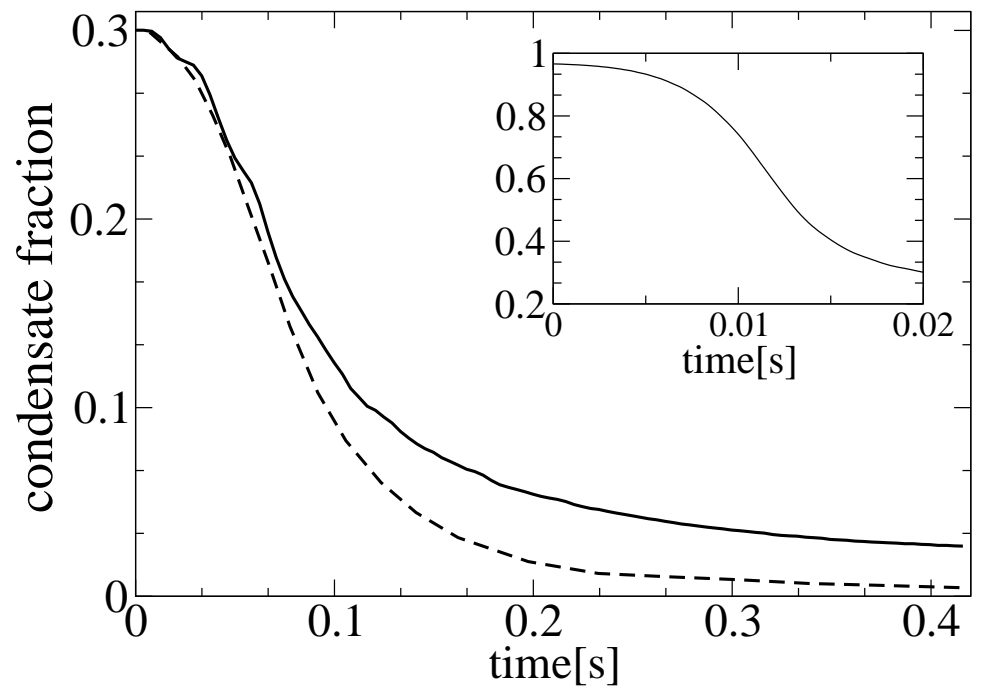

Figure 8. Numerically simulated dynamical transition to the Bose glass state; first (in inset) a superfluid at high value of lattice potential is formed, and then (in the main figure) the disorder is turned on gradually. Condensate (solid line) and superfluid (dashed line) fractions tend to zero (from [68]).

glass phases (weak interaction regime) in a two-dimensional ultracold bosonic gas was calculated using the Gutzwiller ansatz method (see Fig. 8), while in $[69,70]$ the ground state of the system was determined by solving the eigenvalue problem of the one-dimensional Bose-Hubbard hamiltonian numerically. In this last case, also both the Anderson-glass and the Bose-glass phases were found (see Fig. 9).

Very recently [159], the problem of disordered one-dimensional bosonic systems with hard core boson interactions has been exactly solved via a Jordan-Wigner transformation (see subsection 3.8). This mapping establishes a connection to noninteracting disordered fermions and allows to take advantage of many known results on Anderson localization. In this scenario, the correlation functions of the particle density and the local density of states for the interacting bosonic case coincide with the non-interacting fermionic ones, because only depend on the modulus square of the wave-function. This is not the case for the momentum distribution, but the mapping allows for a simple calculation of the disorder-averaged boson momentum distribution [159]. These calculations show the complete destruction of quasi-long range order by disorder and the flattening of the momentum distribution for sufficiently strong disorder. Note that in the homogeneous case, the momentum distribution is well known to possess a singularity [373-375]. In [159] it was also shown that the Bose-Fermi mapping can also be established via the effective low-energy theory [339]. Moreover, it was pointed out that a similar mapping is also available for arbitrary interaction strength, but involves interacting fermions with nonstandard contact interactions [376]. For strong (but finite) repulsive bosonic interactions, the weak fermionic interactions can be treated perturbatively. In Ref. [378], specific effects of quasi-disorder, in contrast to real disorder, in 1D lattices were discussed. 

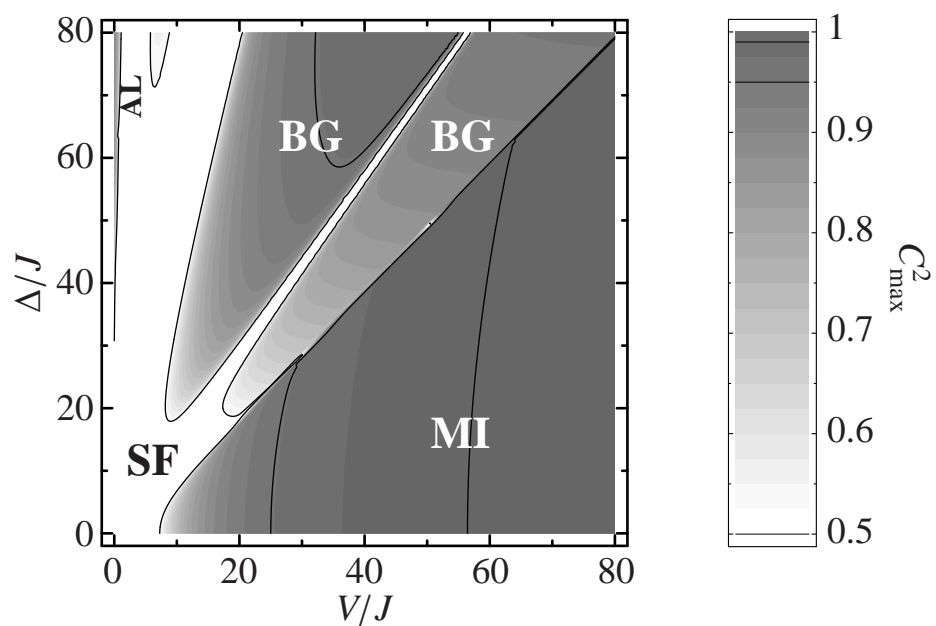

Figure 9. Contour plot of the square of the largest coefficient, $C_{\max }^{2}$, of the expansion of the state of the system described by a Bose-Hubbard model in the number basis, as a function of disorder $\Delta$ and on-site interaction $V$, in units of the hopping energy $J$. The results correspond to exact diagonalization of a one-dimensional system with 8 sites and 8 bosons (from [69]. Note that $V$ accounts for $U$ and $J$ for $t$ in the figure). The labels identify the predicted different phases: superfluid, Mott insulator, Bose glass (BG), and Anderson localization (AL).

Just before submission of this review, Krutitsky et al. [377] calculated the mean field phase diagram of disordered bosons at finite $T$. They made the important observation that the distinction of $\mathrm{MI}$ and Bose glass at finite $T$ in atomic lattice gases should be possible by looking at the density of states of low energy excitations.

The experiments on disordered ultracold gases in optical lattices has just started, and most of them deal with trapped gases and weak interaction limit (we discuss them in the next subsection). Nevertheless, spectacular results have been achieved in the area of disordered lattice gases, already. The Florence group has recently applied to a basic lattice formed by light with wavelength $\lambda_{1}=830 \mathrm{~nm}$ another superlattice created by $\lambda_{2}=1076 \mathrm{~nm}$ light, creating a quasi-disordered potential (see Fig. 10). They have applied the method of Bragg spectroscopy developed in [55] and measured effects of the lattice modulation. This allows essentially to measure the low energy excitation spectrum; broadening of this spectrum was identified in Ref. [35] to be the signature of the Bose glass phase (see Fig. 11).

In the experiment of the Hamburg group [36] (see also [37]), an unexpected shift of the SF-MI transition point towards weaker lattice potentials has been observed in a Fermi-Bose mixture with attractive interspecies interactions. It has been speculated that this effect might have to do with the formation of composite fermions. Composite fermions, consisting of a fermion and few bosons, are hardly mobile and should remain in the conditions of the mentioned experiments immobile and play a role of random scatterers for (still relatively movable) bosons. 


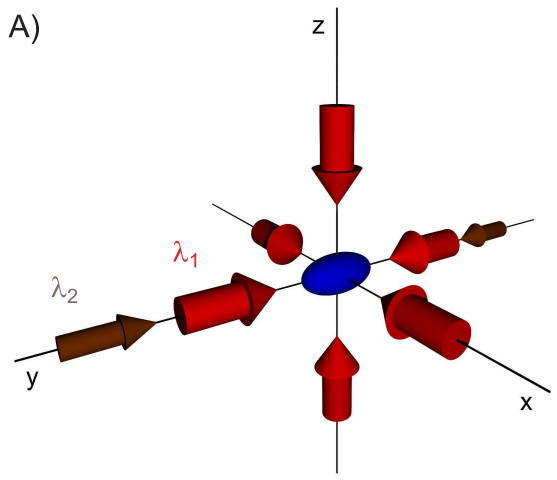

B)

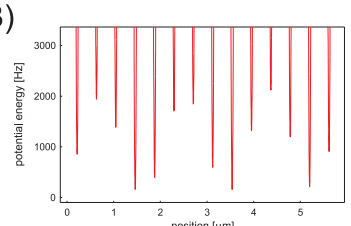

C)

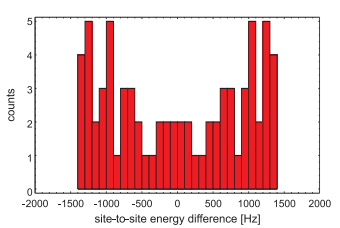

Figure 10. Quasidisorder used in the experiments reported in [35].

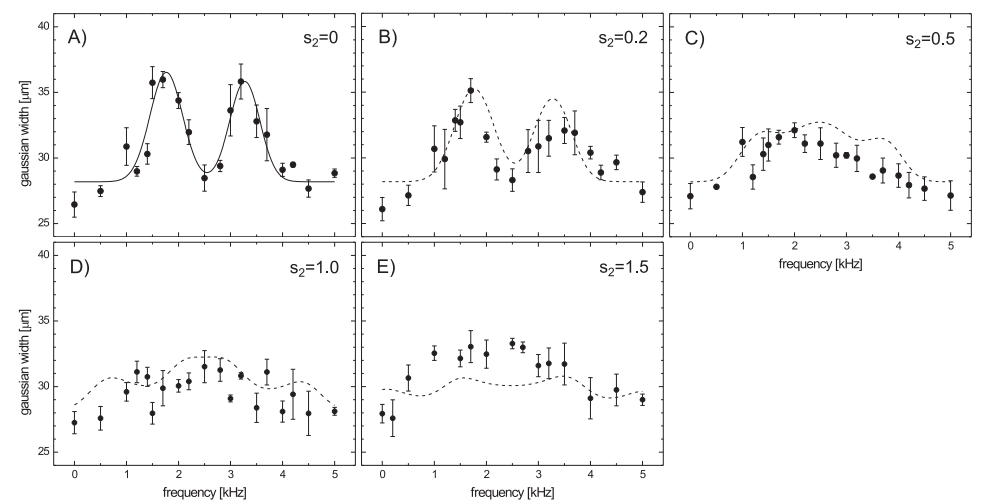

Figure 11. Experimental signatures of the MI-Bose glass transition: Broadening of the excitation spectrum as disorder $\left(s_{2}\right)$ grows. One observes discrete linbes in the gapped MI, and significantly broadened and structureless spectrum in the BG phase (from [35]).

\subsection{Experiments with weakly interacting trapped gases and Anderson localization}

During the last year the first experimental results on Bose-Einstein condensates in random potentials were reported [71-74]. In the first experiments [71], static and dynamic properties of an harmonically trapped ${ }^{87} \mathrm{Rb}$ condensate in the presence of a stationary, but spatially random potential created by imaging an speckle pattern onto the condensate were addressed. In this experiment the smallest length scale of the speckle was $L_{\text {dis }}=10 \mu \mathrm{m}$ while the size of the quasi one dimensional condensate was $110 \mu \mathrm{m}$ in the axial direction and $11 \mu \mathrm{m}$ in the radial. Absorption images after free expansion for different values of the speckle height, $V_{s}$, reveal different regimes of behavior: (i) for strengths of the disorder much smaller than the chemical potential of the condensate in the harmonic trap, practically no change with respect to the standard Thomas-Fermi profile is observed; (ii) for intermediate values of 
$V_{s}$, stripes appear in the expanded density profile, which can be a signature of the development of different phase domains across the condensate, like the ones associated with phase fluctuations in highly elongated condensates [284,379], or they can be explained through the presence of different momentum components due to the growth of instabilities [380]; and (iii) when $V_{s}$ is larger than the chemical potential of the harmonically trapped condensate, the tunneling between the different minima of the random potential is strongly suppressed and the system enters the tight binding regime, where a broad Gaussian profile is seen in the expansion as expected for the expansion of randomly spaced condensates isolated in the individual speckle wells. The transport properties of the system were also studied in [71] through collective excitations, dipolar and quadrupolar modes. The dipolar mode was excited by abruptly displacing the magnetic trap in the axial direction obtaining: (i) for $V_{s}$ much smaller than the chemical potential, slightly damped oscillations were obtained with the same frequency as in the undamped case in the absence of the speckle; (ii) for intermediate $V_{s}$, strong damping of the oscillations is observed; and (iii) for high values of $V_{s}$, the atomic cloud does not oscillate and remains localized on the side of the magnetic trap. The quadrupolar mode was excited with a resonant modulation of the radial trapping frequency observing that the anharmonicities of the random potential result in frequency shifts that are not correlated with the dipolar frequency like it happens in the absence of speckle. Moreover, it is shown that both the sign and the amplitude of the shift depend on the exact realization of the speckle potential.

Following these first experiments, the suppression of the one-dimensional transport of an interacting elongated condensate in a random potential was reported nearly simultaneously by $[72,74]$. In these experiments, one-dimensional expansion of a ${ }^{87} \mathrm{Rb}$ condensate along an optical [72] or magnetic [74] guide in the presence of a speckle potential was studied. Without the speckles, the condensate freely expand and the growth of the root mean squared (rms) radius ( $\mathrm{rms}=\sqrt{\left\langle x^{2}\right\rangle+\left\langle y^{2}\right\rangle}$ ) is self-similar and linear in agreement with the theory [381,382]. In the presence of a random potential with high enough amplitude, but without entering in the tight binding regime, the expansion dynamics changes completely and both the expansion and the centre of mass motion are inhibited. Although this strong suppression of expansion corresponds to disorder-induced trapping of the BEC, it does not correspond to Anderson like localization. The reasons are mainly: (i) the screening of the disorder potential played by the interaction energy; (ii) the resolution of the optics of the disordered potential that fixes the correlation length of the disorder, $L_{d i s}$, which is much larger than the healing length, $l_{\text {heal }}=1 / \sqrt{8 \pi n a}$ where $n$ is the density and $a$ the atomic scattering length. The healing length provides the typical distance over which the order parameter of the condensate recovers its bulk value when it is forced to vanish at a given point by an impurity for instance. Therefore, the density profile tends to follow the modulations of the disordered potential; and (iii) the fixed order of magnitude of the distance between the speckle sites imposes that the typical axial size of the system, $L$, is only ten times larger than the correlation length of the disorder. In order to achieve Anderson localization, the correlation length of the disorder has to be smaller than the size of the system, which seems to be difficult to achieve with speckles. Anderson localisation of elementary excitations in 1D BEC seemingly sets a little less rigid requirement, but is still not easy to achieve with the presently achievable speckle patterns $[77,78]$. Recently, a very detailed study shows that by expanding a quasi $2 \mathrm{D}$ cloud to a size of $1 \mathrm{~cm}^{2}$, it 


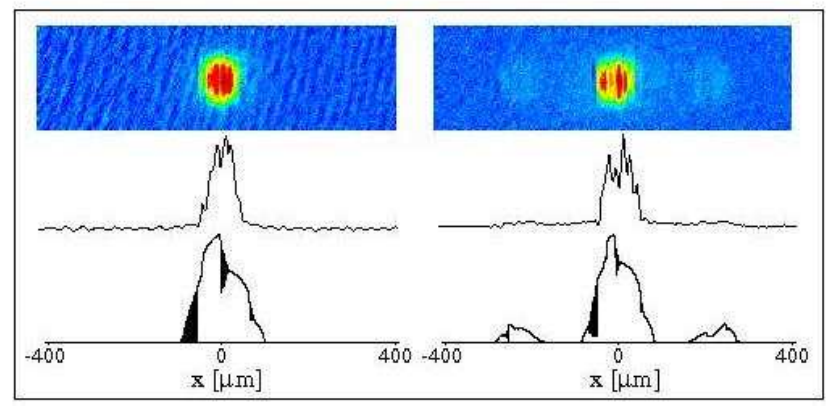

Figure 12. Density profiles (absorption images) of quasi 1D BEC released from the combined harmonic trap plus random speckle (left column), and from the combined harmonic trap, optical lattice, and random speckle (right column). The second row shows the column density and the third row shows the result of the numerical simulation (from [73]).

should be possible to see effects of weak localization and perhaps even strong ones, using available speckle potentials [77].

In Ref. [73], the first realization of an ultracold disordered lattice gas was reported, so the effect of a speckle pattern was superimposed to a quasi-1D condensate in a regular 1D optical lattice. In this work, it was shown that the fragmentation, already reported in [71], also appears in the presence of an optical lattice (see Fig. 12). More interestingly, in [73] it has been explored the crossover from the Anderson localization in the absence of interactions, where the ground state wave function is characterized by an exponential localization, to the screening regime, where the number of localization centres is so high that one can no longer distinguish the individual localized states and the signature of nontrivial localization vanishes (see Fig. 13). On one hand, the interactions can be reduced by reducing the number of atoms, lowering the trap frequencies or tuning the scattering length via Feshbach resonances and on the other hand, the limitations in the ratio between the correlation length of the disorder and the healing length and between the correlation length of the disorder and the size of the system, that arise when the disorder potential is created using an speckle pattern, could be overcome using the quasidisorder created by several lasers with incommensurable frequencies. Measurement of the superfluid fraction in an accelerated optical lattice is proposed [73] as a way of detection of the localization rather than the usual measurement of the density distribution after ballistic expansion. 


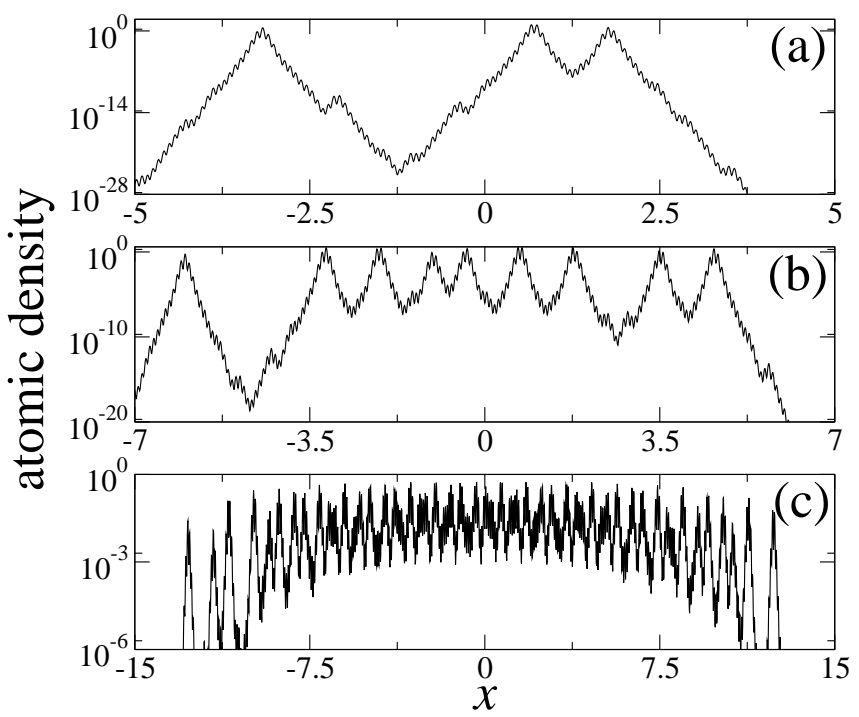

Figure 13. Calculated condensate wavefunction for a BEC in a combined potential formed by harmonic trap, and two incommensurate standing waves. The sequence (a)-(c) shows the effects of the increasing nonlinearity (i.e., increasing the number of atoms) (from [73]).

\subsection{Disordered interacting fermionic systems}

So far we have discussed disordered interacting bosonic systems and the experimental possibilities of testing the still controversial points of the theory using ultracold bosonic systems in optical lattices. Fermionic gases have also been cooled to the quantum degeneracy regime using sympathetic cooling of two fermion species, or boson-fermion mixtures (for the first experiments see [383-387]). Moreover, fermionic ${ }^{40} \mathrm{~K}$ atoms have been also loaded into a three-dimensional optical lattice [34]. Several proposals of using ultracold fermionic atoms in optical lattices to study various condensed matter models have already been formulated. In [90] for instance, it was discussed how fermionic atoms in optical lattices allow for the realization of antiferromagnetism or high-temperature superconductivity. More recently, it has been shown [388] that the fermionic SU(N) Hubbard model on a 2D square lattice can be realized with ultracold fermions in an optical lattice. Regarding the disordered case, very recently it has been reported [389] that fermionic atoms in an optical superlattice exhibit strongly correlated phases from Kondo singlet formation to magnetism of localized spins.

Let us now briefly review the literature on the physics of disordered interacting electronic systems with the aim of identifying the still open questions that could be addressed exploiting the ultracold fermionic atoms in disordered optical lattices.

As we have discussed in subsection 4.1, originally disorder in non-interacting electronic systems attracted a lot of interest, but the effects of electron-electron interactions received full attention only recently. It was believed that weak disorder should not modify essentially the Fermi liquid picture of Landau. Later a Fermi 
liquid theory for electrons in disordered solids was formulated, and termed the Fermi glass theory $[390,391]$. According to this approach the Landau's quasiparticle description is valid but now the quasiparticle wave-functions are not extended, as in the translationally invariant case, but they have no long-range coherence and may be even localized. Each quasiparticle is viewed as a single entity moving in the self-consistent field of all the other quasiparticles, and the resulting theory has the form of a mean-field theory for the quasiparticles. In [391], the phase transition into a Fermi glass state was discussed. In [392-394], and independently in [395], it has been shown, applying perturbation theory to the lowest order in the interaction strength, that even weak disorder leads to surprisingly singular corrections to the electronic density of states near the Fermi surface, and to transport properties. In 1994, Shepelyansky [396] stimulated further discussion about the role of interactions by considering two interacting particles in a random potential, arguing that there exist an interaction-induced enhancement of the two-particle localization length compared to the noninteracting case. The lines of this work were developed further [397-402]. A Fermi liquid approach for finite densities was suggested by [397], and later developed by [400,402] reducing the problem to the study of the delocalization of few quasiparticles above the Fermi sea.

Several groups have tried to study effects of interplay between disorder and (repulsive) interactions in more detail in the regime, when Fermi liquid becomes unstable as the Mott insulator state is approached by increasing the interactions. In other words, the crossover between Fermi glass (Anderson localized states populated with the restriction of the Pauli principle) and Mott insulator (state where repulsion dominates over kinetic energy and disorder) has been studied. Density matrix renormalization group studies were performed for spinless fermions with nearest neighbor (n.n.) interactions in a disordered mesoscopic ring [403], and for spin 1/2 electrons in a ring described by the half-filled Hubbard-Anderson model [404]. Spinless fermions with Coulomb repulsion (reduced to n.n. repulsion) in 2D [405, 406] were also studied. This collection of works shows that as interactions become comparable with disorder, delocalization does takes place. In a 1D ring it leads to the appearance of persistent currents. In 2D, the delocalized state exhibits also an ordered flow of persistent currents, which is believed to constitute a novel quantum phase corresponding to the metallic phase observed in experiments for instance with a gas of holes in GaAs heterostructures for the similar range of parameters.

Another intensive subject of investigation concerns metal (Fermi liquid)-insulator transition driven by disorder in 3D. Theoretical description of this phenomenon goes back to the seminal works of Efros and Shklovskii [407-409] and Mac Millan [410]. In this context, recent results of experiments on disordered alloys $[411,412]$ allowed to determine the critical exponents that govern the conductivity dynamics on both sides of a quantum phase transition in a disordered electronic system. Weakly doped semiconductors provide a good model of a disordered solid, and their critical behavior at the metal-insulator transition has been intensively studied (cf. [413,414]). Results concerning various forms of electronic glass: from Fermi glass (with negligible effects of Coulomb repulsion) to Coulomb glass [415-417], dominated by the electronic correlations were obtained in the group of M. Dressel $[418,419]$.

Very recently, the ground state phase diagram at half filling, for arbitrary interaction and disorder strength, has been calculated [420] by applying the Dynamical Mean-Field Theory (DMFT) with the geometrically averaged local density of 
states. It was shown that the presence of disorder increases the critical interaction at which the Mott-metal transition occurs and turns the sharp transition into an smooth crossover. Regarding the critical disorder strength for the Andersonlocalization transition, it was reported that it increases for weak interactions, and it is suppressed by strong interactions [420]. In the phase diagram obtained with the same method, but with an arithmetic average of the local density of states the Anderson transition is missing [421].

Again, we would like to point out that many of the questions discussed in this section can be addressed using ultracold atomic lattice gases.

\subsection{Disordered Bose-Fermi mixtures}

As mentioned in the subsection 4.6, in order to cool fermionic gases to the quantum degeneracy regime it is necessary to use sympathetic cooling of two spin species or boson-fermion (B-F) mixtures [383-387]. In the latter case, the final phase of the system is a quantum degenerate B-F mixture. The loading of a B-F mixture in an optical lattice has been also recently reported $[36,37,422]$.

In the absence of disorder, and in the limit of strong atom-atom interactions such lattice B-F systems can be described in terms of composite fermions consisting of a bare fermion, or a fermion paired with 1 boson (bosonic hole), or 2 bosons (bosonic holes), etc. [167]. The physics of Fermi-Bose mixtures in this regime has been studied recently in a series of papers [167, 183, 210,218,423,424], where it has been shown that the low temperature dynamics of the fermionic composites is described by an effective Hamiltonian (see also Sec. 3.11 and Appendix A), describing a spinless interacting Fermi gas. The validity of the effective Hamiltonian for fermionic composites in 1D was studied using exact diagonalization and the DMRG method [219]. The effects of inhomogeneous trapping potential on lattice mixtures was for the first time discussed by Cramer et al. [220], while the disordered case in the strong coupling limit was studied in $[86,87]$. In the presence of disorder, degenerate second order perturbation theory cannot be applied to derive the effective Hamiltonian, as it was used in [166, 167], since even for zero hopping rates there exists a well defined single ground state determined by the values of the local chemical potentials. Nevertheless, in general, there will be a manifold of many states with similar energies. The differences of energy inside a manifold are of the order of the difference of chemical potential in different sites, whose random distribution is bounded. Moreover, the lower energy manifold is separated from the exited states by a gap given by the boson-boson interaction. Therefore, one can apply a form of quasidegenerate perturbation theory by projecting onto the manifold of near-ground states, as described in Appendix A [87,230]. As in the homogeneous case [167], composite fermions behave as a spinless interacting Fermi gas, but in the presence of local disorder they interact via random couplings and feel effective random local potential. The effective Hamiltonian that describes their physics can be written as follows:

$$
H_{e f f}=\sum_{\langle i, j\rangle}\left[-d_{i j} F_{i}^{\dagger} F_{j}+h . c .\right]+\sum_{\langle i, j\rangle} K_{i j} M_{i} M_{j}+\sum_{i} \bar{\mu}_{i} M_{i},
$$

where $F_{i}$ are the annihilation operators of the composite fermions (Eqs. (56) and 
(57)), and $M_{i}=F_{i}^{\dagger} F_{i}$. The nearest neighbor hopping for the composites is described by $-d_{i j}$, the nearest neighbor composite-composite interactions is given by $K_{i j}$, which may be repulsive $(>0)$ or attractive $(<0)$, and $\bar{\mu}_{i}$ are the on-site energies. The explicit calculation of the coefficients $d_{i j}, K_{i j}$ and $\bar{\mu}_{i}$ depends on the concrete type of composites fermions [86,87]. For fermion-bosonic hole composite and for fermion-boson composite, it has been shown that the hopping amplitudes $d_{i j}$ are always positive. Depending on the ratio $\alpha=V / U$ between the boson-fermion and the boson-boson interactions, the effective interactions between composites $K_{i j}$ may be either repulsive or attractive for all the values of disorder, or for certain values of $\alpha$ the qualitative character of the interactions is controlled by the inhomogeneity. These two types of composites have been studied in two limits: (i) the small disorder limit, where the contributions of the disorder to the interactions and hopping are neglected $\left(K_{i j}=K\right.$ and $\left.d_{i j}=d\right)$, and only the leading contributions in the onsite energies are kept; and (ii) the large disorder limit or spin glass limit, where the tunneling becomes non-resonant and can be neglected in Eq. (64), while the couplings $K_{i j}$ fluctuate strongly. This situation corresponds to the (fermionic) Ising spin glass model (see Section 4.8 for details).

For the case of disorder applied only for the bosonic component of the mixture, and in the small disorder limit (when disorder does not affect the composite formation), the following quantum phases can be achieved:

- Fermion + bosonic hole composites

When $K / d \ll 1$, i.e., when the interactions are negligible, the system is in the Fermi glass phase, i.e. Anderson localized (and many-body corrected) single particle states are occupied according to the Fermi-Dirac rules. For large repulsive interactions, $K / d \gg 1$ and $K>0$, the ground state will be a Mott insulator and the composite fermions will be pinned for large filling factors. The value of $K / d$ is, however, bounded from above by 2 , which seems not enough to achieve the Mott insulator state. For intermediate values of $K / d$, with $K>0$, delocalized metallic phases with enhanced persistent currents are possible. For attractive interactions $(K<0)$ and $|K| / d<1$ one expects competition between pairing of fermions and disorder, i.e., a "dirty" superfluid phase while for $|K| / d \gg 1$, the fermions will form a domain insulator, that is a state in which fermionic composites will stick together to form a rigid immobile cluster. In Ref. [87] the crossover from the Fermi gas to the Fermi glass phases has been studied numerically by means of the dynamical Gutzwiller ansatz method (Fig. 14); similarly, dynamics of a transition from the fermionic domain insulator to a disordered insulating phase was investigated (Fig. 15).

- Fermion + boson composites

The regimes where $K \ll d$ lead to a non-interacting Fermi glass while the regimes of strong effective repulsive interactions, where $K \gg d$ and $K>0$, would correspond to Mott insulator or checkerboard phase if the filling factor is $1 / 2$. In this case, no strong attractive interactions regime occurs since $K / d$ reaches a minimum of $\simeq-0.07$. Therefore, the domain insulator phase does not appear, and even the "dirty" superfluid phase may be washed out.

The summary of the possible phases, described in the case of fermions plus bosonic hole composites, and in the case of fermion plus boson composites, is presented in the schematic diagram of Fig.16. In the case of bare fermion composites, it has been shown that for finite boson-fermion interactions, the fluctuations of the 

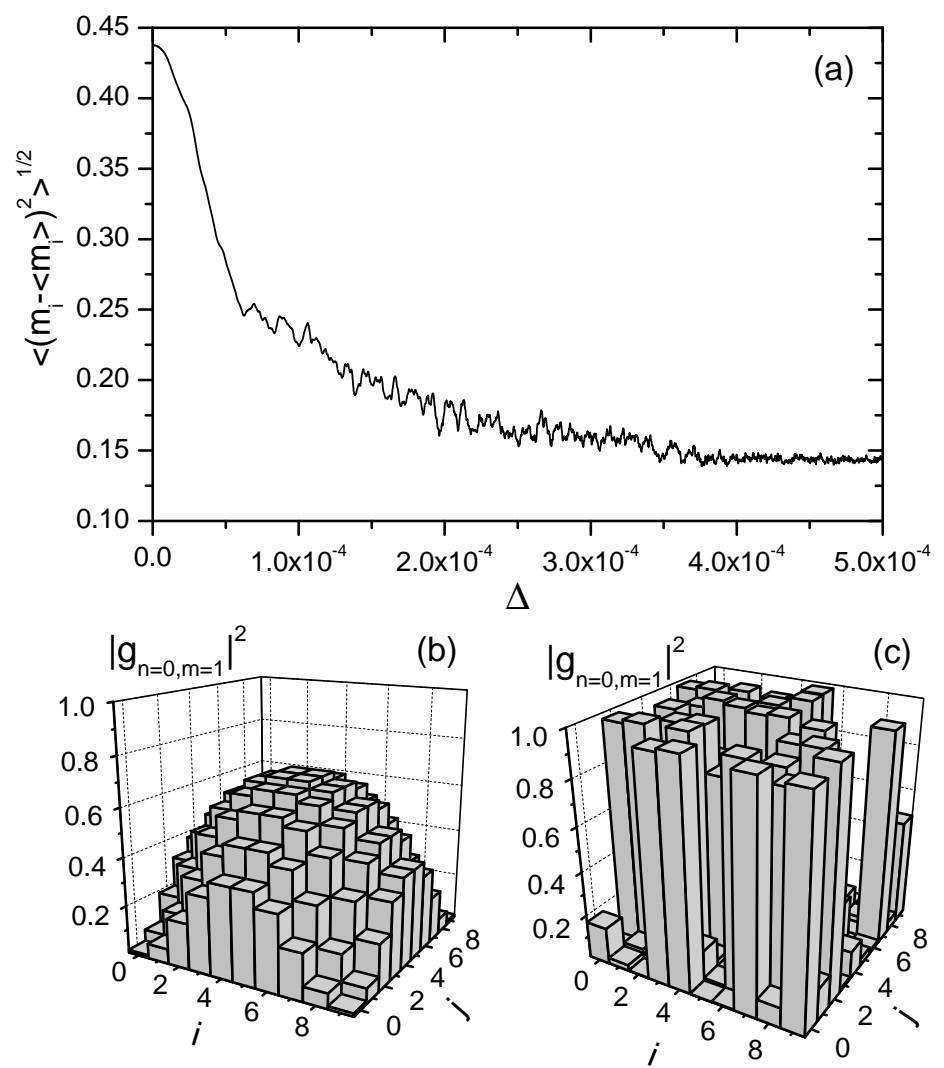

Figure 14. Numerically calculated dynamical transition from the Fermi liquid to the Fermi glass of fermionic composites (fermion + bosonic hole composites) in a 2D lattice with $N=10 \times 10$ sites. In (a), the decrease of the variance of the number of fermions per lattice site is shown as a function of the amplitude of the disorder. In (b), the probability of having one composite at each lattice site in the absence of disorder is given, and (c) gives the same as (b) after adiabatically ramping up the disorder (from [87]).

effective composite interactions may be large, and the dynamics of this type of composites resembles quantum bond percolation. One can assume, in a somehow simplified view, that the interaction parameter $K_{i j}$ takes either very large, or zero values. The lattice decomposes then into two sub-lattices: a "weak" bond sub-lattice (corresponding to $K_{i j} \ll d_{i j}$ ) in which fermions flow as in an almost ideal Fermi liquid, and a "strong" sub-lattice (corresponding to $K_{i j} \gg d_{i j}$ ), where only one fermion per bond is allowed. Additionally, for the case of lattices with different types of sites (i.e. sites in which disorder affects the formation and character of composites), it has been predicted that physics of quantum site percolation will become relevant. 


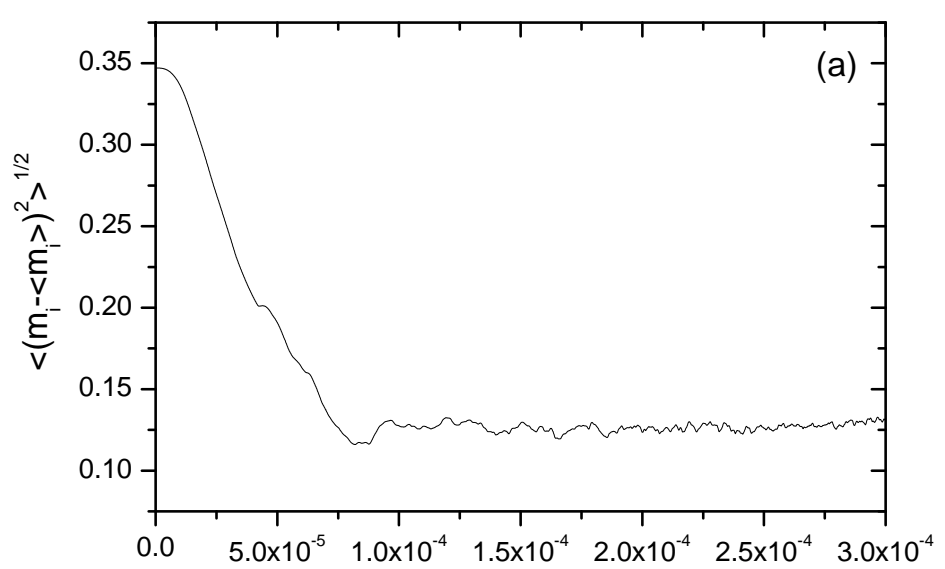

$\Delta$
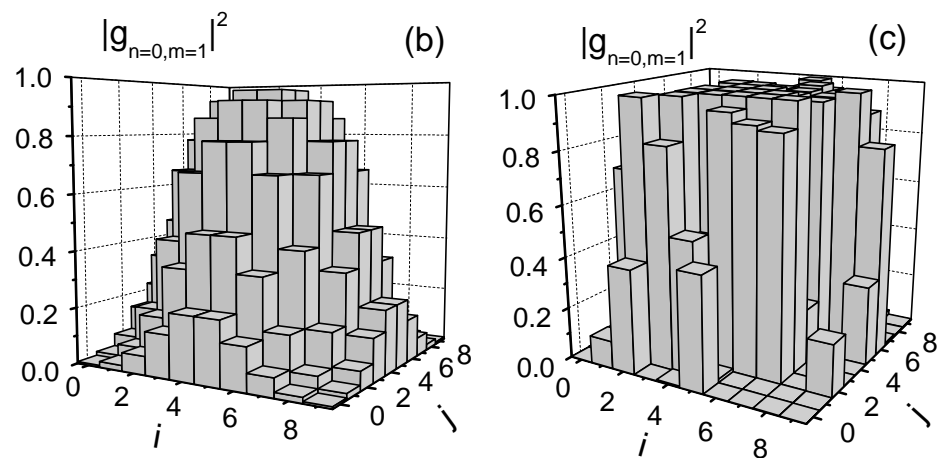

Figure 15. Numerically calculated dynamical transition from the Fermi domain to the Fermi glass of fermionic composites (one fermion and zero boson) in a 2D lattice with $N=10 \times 10$ sites. In (a), the decrease of the variance of the number of fermions per lattice site is plotted as a function of the amplitude of the disorder. In (b), the probability of having one composite at each lattice site in the absence of disorder is given, and (c) gives the same after adiabatically ramping up the disorder (from [87]).

\subsection{Spin glasses}

Spin glass is a phase that appears in spin systems interacting via random couplings that can be positive (anti-ferromagnetic), or negative (ferromagnetic). Such variations of the couplings lead typically to frustration i.e., if there are only two possible spin orientations and the interactions are random, no spin configuration can simultaneously satisfy all the couplings (see section 5 for more details). Thus, spin glass behavior requires two essential ingredients: quenched disorder and frustration.

In a somewhat oversimplified picture, this kind of systems are characterized by two order parameters: (i) the order parameter for magnetic ordering, the magnetization given by $M:=<\overline{\sigma_{i}}>$ and (ii) the Edwards-Anderson order parameter for spin glass 
(a)

\begin{tabular}{llllll}
$\begin{array}{l}\text { Domain } \\
\text { insulator }\end{array}$ & $\begin{array}{l}\text { "Dirty" } \\
\text { superconductor }\end{array}$ & $\begin{array}{l}\text { Fermi } \\
\text { Glass }\end{array}$ & $\begin{array}{l}\text { Metallic } \\
\text { Phases }\end{array}$ & \\
\hline$>$ 1 and $<0$ & $\sim-1$ & $\mathbf{0}$ & $\sim \mathbf{1}$ & $\mathbf{2}$ & $\mathbf{K} / \mathbf{d}$
\end{tabular}

(b)

\begin{tabular}{cccc}
$\begin{array}{c}\text { Fermi } \\
\text { Glass }\end{array}$ & $\begin{array}{l}\text { Metallic } \\
\text { Phases }\end{array}$ & $\begin{array}{l}\text { Mott Insulator } \\
\left(\text { Checker-board for } \rho_{\mathrm{f}}=1 / 2\right)\end{array}$ \\
\hline $\mathbf{0}$ & $\sim \mathbf{1}$ & $\mathbf{> 1}$ and $>\mathbf{0}$ & $\mathbf{K} / \mathbf{d}$
\end{tabular}

Figure 16. Schematic predicted phase diagram of (a) fermion plus bosonic hole composites and of (b) fermion plus boson composites [87]).

ordering, $Q_{E A}:=\overline{\left\langle\sigma_{i}\right\rangle^{2}}$, where $\overline{(\cdot)}$ denotes the average over disorder and $\langle(\cdot)\rangle$ the thermal average. Experimental studies have identified the phase diagram of the spin glasses consisting on three phases [425]: (i) At high temperature and small average spin exchange, $\bar{K}$, the system exhibits a paramagnetic phase with $M=0$ and $Q_{E A}=0$; (ii) for $\bar{K}<0$ and large, the system exhibits a ferromagnetic phase with $M \neq 0$ and $Q_{E A} \neq 0$; and (iii) for weak $\bar{K}$ and small temperatures, a spin glass phase appears with $M=0$ and $Q_{E A} \neq 0 . Q_{E A} \neq 0$ signals that the local magnetization is frozen, but may vary from site to site, so that the disorder prevents long range magnetic order. Spin glass phase is an example of "order in the presence of disorder" [425].

The physics of spin glasses is up to now not well understood and remains as one of the challenges of statistical physics. In particular, the question of the nature of their order is still open. There exist two competing theories: the Mézard-Parisi (MP) model, and the droplet model. The MP picture is a mean-field theory based on the replica method [83] that predicts that the spin glass phase consists of a large number of low-energy states with very similar energies. The applicability of the MP picture for short range spin glasses (like the Edwards-Anderson model [81]) is very questionable. The rival theory, the droplet model $[84,85,332,426,427]$ is a phenomenological theory based on scaling arguments and numerical results. The droplet model predicts that there are two ground states related by spin-flip symmetry, and that excitations over the ground state are regions with fractal boundary, the droplets, in which the spins are inversed with respect to the ground state.

One of the most remarkable results of Refs. $[86,87]$ is the possibility of the realization of a fermionic Ising spin glass model by using a disordered Bose-Fermi mixture. The spin glass limit is obtained from the composite fermionic model (Eq.(64)) when 
the interactions between fermions and bosons are of the same order, but slightly smaller than the interactions between bosons, and in the limit of large disorder. In this situation, the hopping vanishes due to strong site-to-site energy fluctuations and the nearest neighbor interactions, $K_{i j}$, fluctuate around mean zero with random positive and negative values. Replacing the composite number operators with a classical Ising spin variable $\sigma_{i}:=2 M_{i}-1= \pm 1$, one ends up with the Hamiltonian:

$$
H_{E-A}=\frac{1}{4} \sum_{\langle i j\rangle} K_{i j} \sigma_{i} \sigma_{j}+\frac{1}{2} \sum_{i} \bar{\mu}_{i} \sigma_{i} .
$$

It describes an (fermionic) Ising spin glass [428,429], which differs from the standard Edwards-Anderson model [83,430] in that the model (Eq.(65)) includes an additional random magnetic field $\bar{\mu}_{i}$, and, moreover, it has to satisfy the constraint of fixed magnetization value, $m=2 N_{f} / N-1$, as the number of fermions, $N_{f}$, in the underlying BFH-model is conserved. $N$ is the number of sites. It shares, however, the basic characteristics with the Edwards-Anderson model as being a spin Hamiltonian with random spin exchange terms $K_{i j}$. In particular, this provides bond frustration, which in this model is essential for the appearance of a spin glassy phase. Due to the mentioned differences, it is necessary to reformulate slightly the Mézard-Parisi mean field description of the system by adapting the Sherrington-Kirkpatrick-Parisi calculations [82,83] to this specific case [87].

The experimental study of this limit in ultra-cold Bose-Fermi mixtures could present a way to address various open questions of spin glass physics concerning the nature and the ordering of its ground- and possibly metastable states (the Mézard-Parisi picture [83] versus the droplet picture [84, 85, 332,426,427]), broken symmetry and dynamics in classical (in absence of hopping) and quantum (with small, but nevertheless present hopping) spin glasses [154,431]. Particularly interesting are prospects for studying questions that have been addressed only recently: the existence of the celebrated de Almeida-Thouless line (i.e. the line separating the replica symmetry broken spin glass phase from the replica symmetric phase in the magnetic field in the Parisi picture) in short range spin glasses [432, 433], disorder chaos in spin glasses (i.e. ground state sensitivity to small changes of disorder, [434]), spin glass transition in 2D and its dependence on the bond distribution [435], universality classes [436] etc. (for a recent review see [366]).

Usage of atoms offers unprecedented possibilities for detection of the spin glass properties. Recently for instance, it has been proposed [437] how to create replicas of disordered systems, i.e. systems with identical disorder landscape and to the measure of correlations between the replicas. These correlations can provide information about the ground state phases of the system. The replicas can be created in a three dimensional lattice with incommensurate frequencies by quenching the hopping between different planes obtaining then a set of two-dimensional incommensurate lattices, with the same realization of disorder in each plane. Another possibility is to use localized impurity atoms. In this case, the procedure would be first to prepare a disordered distribution of localized impurity atoms in such a way that only two or no atoms per lattice site are allowed. This can be achieved, for instance, using repulsive bound pairs, observed recently by the Innsbruck group [39]. Then, periodicity of the lattice in one direction should be adiabatically doubled by stretching the lattice in that direction. One would in this way obtain an array of 


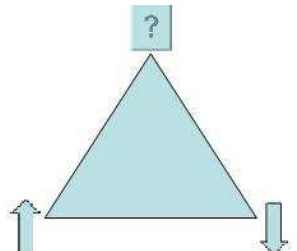

(a)

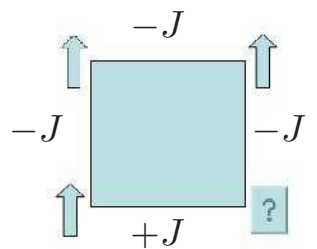

(b)

Figure 17. (a) Antiferromagnetic Ising model $(J>0)$ on a triangular lattice: Orientation of a spin cannot be determined by satifying all the couplings simultaneously. (b) Square lattice with all ferromagnetic Ising interactions, except one, in which the interaction is antiferromagnetic. The interactions are indicated on the sides of the lattice. The model is again frustrated.

replicated pairs of $2 \mathrm{D}$ random landscapes. That would be, obviously, an ideal tool to study the questions concerning existence of finite $T$ spin glass transition in Ising model with bimodal bond distribution [366].

Refs. $[86,87]$ demonstrate that ultracold disordered Bose-Fermi mixtures in optical lattices may serve as a paradigm fermionic system to study a variety of disordered phases and phenomena: from Fermi glass to quantum spin glass and quantum percolation. No doubts, one can mimic condensed matter and even go beyond!

\section{$5 \quad$ Frustrated models in cold atom systems}

\subsection{Introduction}

Frustration appears when all the constraints imposed by the Hamiltonian cannot be simultaneously fullfilled and it is an inherent property of some strongly correlated systems. It introduces various interesting features, such as a rich phase diagram. Particularly fascinating effects appear when a system is both disordered and frustrated (see, e.g., $[83,154]$ ). In this section, however, we will study several frustrated models in regular lattices and discuss their possible realizations. Antiferromagnetic (AF) models in a regular lattice are frustrated if the geometry of the lattice is sufficiently complicated (such as triangular or kagomé lattice).

A physical system described by the Ising Hamiltonian

$$
H_{I}=J \sum_{\langle i j\rangle} \sigma_{i}^{z} \sigma_{j}^{z}
$$

is AF, if $J>0$, where $\langle i j\rangle$ denotes the summation over nearest neighbors. This model, when considered on a two-dimensional triangular lattice, is an example of a frustrated system [438]. It is clear from Fig. 17(a) that it is not possible to minimize the energy on such a triangular lattice, satisfying all the bonds simultaneously. In this case, we obtain a degeneracy in the ground state due to the frustration. It is not due to the invariance under global spin flips of the Hamiltonian, which is the case for the ferromagnetic Ising model $(J<0)$ on the same lattice.

Frustration can be met even in a lattice, which has a relatively simple structure (e.g., a 2D square lattice), if some interactions are ferromagnetic while others are 
AF. In the ferromagnetic Ising model, e.g., if one replaces an odd number of ferromagnetic bonds by AF ones, one obtains a frustrated model from a nonfrustrated one. The square lattice in Fig. 17(b) is an example of such a model. In general, we say that the model is frustrated, when the orientations of its spins cannot be obtained by satisfying all the couplings simultaneously to the aim of minimizing the energy in the model [83]. Further discussion on this subject can be found in [107]. Detailed studies of quantum antiferromagnets can be found in the books of Auerbach [60] and Sachdev [154]. Frustrated models have been reviewed very recently by Misguich and Lhuillier [107] (see also [108,109]). They have been also discussed in the context of spin glasses (see [430]). The concept of frustration has been also studied from the perspective of high temperature superconductors (e.g., see the reviews [439] and [440]).

The plan of this section is as follows. We will present a short review on quantum antiferromagnets in the succeeding subsection. The next two subsections (5.3 and 5.4) will be devoted to the kagomé lattices: we will review some recent results and discuss possibilities of experimental verifications of the theoretical predictions. In the last subsection, we will discuss the possible realization of quantum magnets with cold atoms, ions, and molecules.

\subsection{Quantum antiferromagnets}

In this section we discuss some basic properties of the quantum antiferromagnets. The interest in this subject is motivated mainly by two things: (a) frustrated quantum antiferromagnets are related to most challenging open questions of condensed matter physics; (b) there exist already several theoretical proposals for experimental realization of quantum magnets in cold atom/ion systems. We expect that these experiments will verify existing theoretical predictions, and point out new directions in theoretical investigations of the quantum magnetism.

According to reviews [107-109], quantum antiferromagnets exhibit at low temperatures one of four generic behaviors (see below). We add to the list a fifth possibility $[110,111]$.

(i) Néel order. This is a standard up-down-up-down type of ordering that occurs often in regular lattices such as square in 2D or cubic in 3D. Generalized (planar) Néel order might, however, occur also in frustrated antiferromagnets, for instance in the triangular lattice [441]. Néel order breaks rotational and translational symmetry, exhibits long range order, and leads to gapless spin wave excitations.

(ii) Valence bond solids (VBS). This order breaks the lattice translational symmetry and consists of an ordered covering of the lattice by singlets, called also dimers. There is a long range order in dimer correlations, but the system is gapped and correlation functions decay exponentially. A famous example of a VBS is the Affleck-Kennedy-Lieb-Tasaki (AKLT) state, although it does not break the lattice translational symmetry [60]. Excitations in 2D VBS are gapped (confined spinons) [109].

(iii) Spin liquid of the I type. This order has no apparent symmetry breaking, and decaying correlations. Particularly interesting are topological spin liquids $[109,442]$, for which degeneracy of the ground state depends on the topology of the underlying lattice. 
(iv) Spin liquids of the II type. This order has no symmetry breaking, is gapless, and has large density of states of low energy excitations. Heisenberg antiferromagnet in trimerized kagomé lattice, discussed below is an example of such behavior, representing in a resonating valence bond state (RVB). The concept of resonating valence bond liquid was first introduced by Anderson [443], in the context of superconductivity. When a valence bond solid starts melting due to quantum fluctuations introduced in the system, the situation gives rise to a new phase called resonating valence bond liquid. Numerical Monte Carlo simulations suggest that the liquid does not show any long-range correlations [444], and is a products of singlets [109].

(v) Quantum spin-liquid crystal. This order combines Néel order with spin liquid type II behavior. Numerical studies reveal [110-112] that it has a large number of low energy excitations as in the spin-liquid type II, and presumably no gap.

5.2.1 The Heisenberg model. Let us first focus on spin systems that do not show frustration. Introduction of such models will help us to explain many results in the frustrated models. Let us consider the Heisenberg model

$$
H_{H}=J \sum_{\langle i j\rangle} \overrightarrow{\sigma_{i}} \cdot \overrightarrow{\sigma_{j}}
$$

where $\vec{\sigma}$ is the vector of spin- $1 / 2$ Pauli operators.

The ferromagnetic case. For the sake of completeness, we first look at the $2 \mathrm{D}$ ferromagnetic model $(J<0)$ in a square lattice. The exact ground state is given by the state [155]

$$
\left|\psi_{F}^{G}\right\rangle=\prod_{i}|0\rangle_{i}
$$

where $|0\rangle_{i}$ and $|1\rangle_{i}$ represent respectively the up and down eigenstates of $\sigma^{z}$ at the $i$-th site.

The antiferromagnetic case. In the case of 2D antiferromagnets $(J>0)$ on the square lattice, the exact ground state is hard to find though the system is not frustrated. Nonetheless, the classical ground states, obtained after replacement of the $\vec{\sigma}$ operators by vectors, can be explicitly written down. To this aim the lattice is divided into two sublattices $\mathrm{A}$ and $\mathrm{B}$ in such a way that all nearest neighbors of sublattice A belong to the sublattice B, and vice-versa. Such a splitted lattice is called a bipartite lattice. The classical ground state can be obtained by taking some orientation (e.g., in the z-direction) of all spins in one sublattice and opposite orientation of all spins in the other sublattice. In this way, one can find the classical ground state of a square lattice $[154,445]$ :

$$
\left|\psi_{c l, A F}^{G}\right\rangle=\prod_{i \in A, j \in B}|0\rangle_{i}|1\rangle_{j} .
$$

This classical ground state is known as the Néel state. Note here that any pair of spins in this classical ground state is either parallel or antiparallel, which means 
that the ordering is collinear. The ordering of classical ground states in frustrated systems, however, is noncollinear, even in bipartite lattices [154].

In the case of one-dimensional AF model, the exact ground state of the spin- $1 / 2$ antiferromagnet in the thermodynamic limit is known (Bethe solution) $[60,294,446$, 447]. For comparing the properties of integer and half-integer spin systems [448] (cf. [449]), and the famous Haldane conjecture, the systems may be realized with atoms either by using Fermi-Fermi mixtures, or by employing the approach of Ref. [62].

For quantum antiferromagnets, made up of spin- $1 / 2$ particles, and placed in the infinite square lattice, the ground state energy can be approximated by that of the Néel state (Eq.(69)), despite the fact that it is not an eigenstate of the quantum Hamiltonian (Eq.(67)).

5.2.2 The $\boldsymbol{J}_{\mathbf{1}}-\boldsymbol{J}_{\mathbf{2}}$ model. Let us now move on to the $J_{1}-J_{2}$ model that can be frustrated for some values of interaction couplings, and is a paradigmatic example of VBS. The Hamiltonian of this model can be written as

$$
H_{J_{1} J_{2}}=2 J_{1} \sum_{\langle i j\rangle} \overrightarrow{\sigma_{i}} \cdot \overrightarrow{\sigma_{j}}+2 J_{2} \sum_{\langle\langle i j\rangle\rangle} \overrightarrow{\sigma_{i}} \cdot \overrightarrow{\sigma_{j}},
$$

where $\langle\langle i j\rangle\rangle$ denotes the next nearest neighbors. This is a frustrated model (even in the case of a linear chain), when both $J_{1,2} \neq 0$ and at least one of them is positive. As will be discussed in Sec. 5.3, this Hamiltonian can also be obtained using Fermi-Fermi mixtures in an optical lattice. Its importance comes from its usefulness in explanation of magnetic properties of $\mathrm{Li}_{2} \mathrm{VOSiO}_{4}$ and $\mathrm{Li}_{2} \mathrm{VOGeO}_{4}$ compounds [450]. Below, we will discuss the different phases of this model.

The one-dimensional system. The Hamiltonian (Eq.(70)) for the case of the $1 \mathrm{D}$ lattice, with an even number of sites, periodic boundary conditions, and $J_{1}=$ $2 J_{2}>0$ is known as the Majumdar-Ghosh model [451]. The ground state space of the model is spanned by the two dimers ${ }^{1}$

$$
\left|\psi_{M G}^{G}\right\rangle_{ \pm}=\prod_{i=1}^{N / 2}\left(|0\rangle_{2 i}|1\rangle_{2 i \pm 1}-|1\rangle_{2 i}|0\rangle_{2 i \pm 1}\right) / \sqrt{2}
$$

where $N$ is the number of sites in the lattice. We also call these singlet states as "valence bond states". Note that unlike the AKLT state, this model breaks translational symmetry. A "valence bond solid" is formed from these valence bond states after they order between themselves.

The two dimensional model. The $2 \mathrm{D}$ case is far more complicated. The quantum phase diagram of this model is not clear even for the square lattice, while the classical phase diagram is quite well-understood [107]. In the latter case, it is known

\footnotetext{
${ }^{1}$ The Majumdar-Ghosh model is one of the few frustrated spin models for which the exact ground states are known. See also, e.g. [452-455].
} 


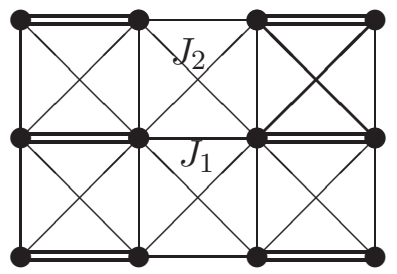

Figure 18. The $J_{1}-J_{2}$ model with dimer configurations in a square lattice. The double lines indicate singlets between the two corresponding spins. The interaction strength corresponding to the diagonals of the squares are $J_{2}$, while that of the sides are $J_{1}$.

that when $J_{2} / J_{1}$ is very small, the system is Néel ordered, and in the opposite extreme (i.e., $J_{2} \gg J_{1}$ ), it has collinear ordering (different than Néel) characterized by

$$
\left|\Psi_{c l, J_{2} \gg J_{1}}^{G}\right\rangle=\prod_{i} \prod_{\text {odd } j}|1\rangle_{i j} \prod_{\text {even } j}|0\rangle_{i j},
$$

where $i$ and $j$ are respectively the indices of rows and columns of the 2D lattice. However, when $0.4<J_{2} / J_{1}<0.6$ (the strongly frustrated regime), no such orderings exist.

These classical predictions are in qualitative agreement with semi-classical and fully quantum calculations based on: series expansion [456-460] (see [461,462] for an introduction to this method), spin wave theory [463,464] (first introduced by Anderson [465], and then extended to higher order by Kubo [466] and Oguchi [467]), exact diagonalizations [468-470], and Quantum Monte Carlo [471].

In the semi-classical limit, spin wave theory predicts a first-order phase transition from the Néel ordered phase to the collinear ordered phase (in the highly frustrated region at $\left.J_{2} / J_{1}=0.5\right)$. However, when quantum fluctuations are introduced in this region, a new phase will appear to separate these two phases. From the exact diagonalizations $[456,472,473]$ one obtains that there are two phase transitions. One is at $J_{2} / J_{1} \approx 0.38$ and another one is at $J_{2} / J_{1} \approx 0.6$. The first one is a second order phase transitions from the Néel state to a spin liquid valence bond state. The second transition is a first order phase transition from the spin liquid state to a collinear state. In the regime $0.38<J_{2} / J_{1}<0.6$, many calculations [474-480] suggest that the ground state may have the VBS dimer configuration with long range ordering, as shown in Fig. 18.

Despite all these efforts, the phase diagram of this model is still not completely understood. In particular, many candidates for the ground states are proposed for this highly frustrated regime. For example, the variational approach suggests [481] that a ground state is a spin liquid resonating valence bond state for a spin- $1 / 2$ system. Sushkov et al. [482] (see also [468]) found two new second-order phase transitions by using series expansion method: one of them around $J_{2} / J_{1}=0.34 \pm$ 0.04 , while the other in the strongly frustrated regime (at $J_{2} / J_{1}=0.50 \pm 0.02$ ). 

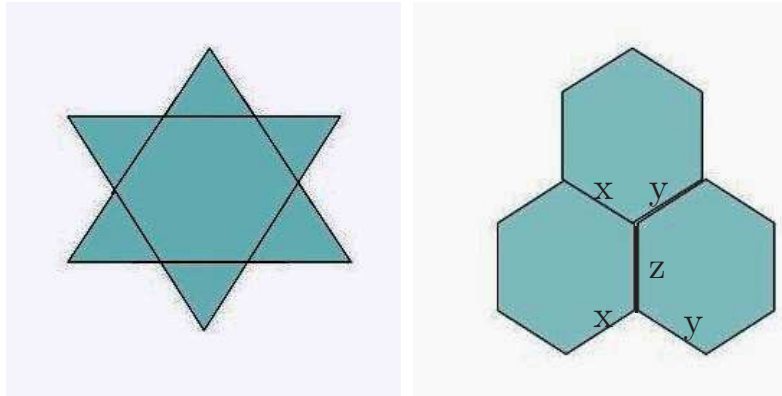

Figure 19. (a) (Left) The kagomé lattice. Two parent triangles superimposed on each other. Each of the parent triangles (one of which is inverted) contains three triangles at its three corners. The triangles in one of the parent triangles (say, in the non-inverted one) have interaction couplings $J$, while those in the other have $J^{\prime}$, between all their vertices. The kagomé lattice consists of a $2 \mathrm{D}$ array of such superimposed parent triangles. (b) (Right) The honeycomb lattice for a spin- $1 / 2$ system, whose Hamiltonian is given in Eq. (78). The three types of links are depicted in the figure.

\subsection{Heisenberg antiferromagnets and atomic Fermi-Fermi mixtures in kagomé lattices}

In the preceding subsection, we have discussed quantum magnets either in a linear chain or in a square lattice. In this section, we move to a more complicated lattice: the kagomé lattice. The Heisenberg antiferromagnet in this lattice is frustrated.

A schematic diagram of the kagomé lattice is depicted in Fig. 19. During the last 15 years, extensive work have been done on this model with Ising-type nearest neighbor interactions [438,483], or with Heisenberg-type nearest and next-nearest neighbor interactions [484-494]. All that give us a lot of information about the ground and excited states of these models. There are still, however, many questions to be answered.

5.3.1 Heisenberg kagomé antiferromagnets. The Hamiltonian, in this case, is given by

$$
H_{k a g}=J \sum_{\langle i j\rangle} \overrightarrow{\sigma_{i}} \cdot \overrightarrow{\sigma_{j}}+J^{\prime} \sum_{\langle i j\rangle} \overrightarrow{\sigma_{i}} \cdot \overrightarrow{\sigma_{j}}
$$

where $J$ and $J^{\prime}$ are the couplings (see caption of Fig. 19). Both $J$ and $J^{\prime}$ are positive.

Numerical simulations of the spin-1/2 system on the kagomé lattice suggest that the energy gap between the ground state and the lowest triplet state, if any, is very small (of order $J / 20$ ). This gap is filled with low-lying singlets, whose number scales with the number of spins, $N$, as $1.15^{N}$ [484]. All these results suggest that this model may be described by the resonating valence bond states.

In the trimerized limit, i.e., when the ratio $J^{\prime} / J$ is very small, Mila and Mambrini $[492,493]$ have found the number, the form, and the spectrum of singlets by using mean field approximation. It corresponds to short-ranged resonating valence bond states. This method also predicts a gap of $2 J^{\prime} / 3$ between singlet and triplets, and the gap remains in the thermodynamic limit. In the fully trimerized limit (i.e., $\left.J^{\prime} / J=0\right)$, the ground state in the subspace of short-ranged resonating valence bond 
states, is one singlet in each of the triangles. Although there are many theoretical predictions about this model, there are no clear experimental confirmations. We will now consider a possible way of verifying these theoretical results by using ultracold atoms [63].

5.3.2 Realization of a kagomé lattice by Fermi-Fermi mixtures. Consider a Fermi-Fermi mixture at $1 / 2$ filling for each species $[63,111]$. The Hamiltonian in this case is the spin- $1 / 2$ Hubbard model, given by

$$
H_{F F}=-\sum_{\langle i j\rangle} t_{i j}\left(f_{i}^{\dagger} f_{j}+\tilde{f}_{i}^{\dagger} \tilde{f}_{j}+\text { h.c. }\right)+\sum_{i} V n_{i} \tilde{n}_{i},
$$

where $n_{i}=f_{i}^{\dagger} f_{i}\left(\tilde{n}_{i}=\tilde{f}_{i}^{\dagger} \tilde{f}_{i}\right)$, and the operators $f_{i}$ and $f_{i}^{\dagger}\left(\tilde{f}_{i}\right.$ and $\left.\tilde{f}_{i}^{\dagger}\right)$ are the annihilation and creation operators for the two species. Here $t_{i j}$ takes the value $t_{0}$ for intratrimer, and $t_{0}^{\prime}$ for intertrimer hopping. In the strong coupling limit, $t_{0}, t_{0}^{\prime} \ll$ $V\left(t-J\right.$ model) [60], $H_{F F}$ reduces to the Heisenberg antiferromagnet Hamiltonian $H_{\text {kag }}$ (Eq. (73)), where $J=4 t_{0}^{2} / V$, and $J^{\prime}=4 t_{0}^{\prime 2} / V$, and $\vec{\sigma}=\left(\sigma^{x}, \sigma^{y}, \sigma^{z}\right)$, with

$$
n-\tilde{n}=2 \sigma^{z}, \quad f^{\dagger} \tilde{f}=\sigma^{x}+i \sigma^{y}, \quad \tilde{f}^{\dagger} f=\sigma^{x}-i \sigma^{y} .
$$

The total spin in the trimer takes the minimal value $1 / 2$, and there are four degenerate states having $\sigma^{z}= \pm 1 / 2$ and left or right chirality. The spectrum of the system in the singlet sector consists of a narrow band of low energy states of a width of the order $J^{\prime}$, separated from the higher singlet (triplet) bands by a gap of the order $3 J / 4\left(2 J^{\prime} / 3\right)$.

\section{$5.4 \quad$ Interacting Fermi gas in a kagomé lattice: Quantum spin-liquid crystals}

In this section we show that an interacting Fermi gas placed in a trimerized kagomé lattice behaves as a very special quantum magnet, a quantum spin-liquid crystal, possessing both antiferromagnetic order and an exceptionally large number of lowenergy excitations $[110,111]$.

The trimerized kagomé lattice can be created by a proper superposition of standing laser beams $[63,111]$. Such a lattice consists of trimers (i.e., sets of three closely packed potential minima) arranged in a perfect triangular pattern (see Fig. 20(a)). The ultracold fermions that are loaded into the lattice stay localized around potential minima, and the Fermi-Hubbard model can be shown to capture all essential properties of this system. The density of fermions is assumed to be such that there are two fermions per each trimer. It is also assumed that there is a long-range interaction between fermions at nearest neighbouring sites in nearest neighbour trimers. This long-range interaction can be achieved, e.g., in a gas of polarized dipolar fermions. 
(a)

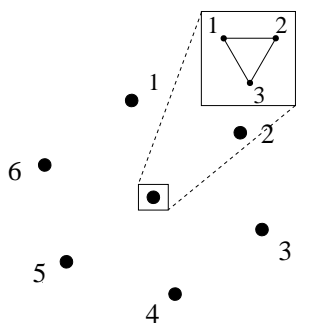

(b)

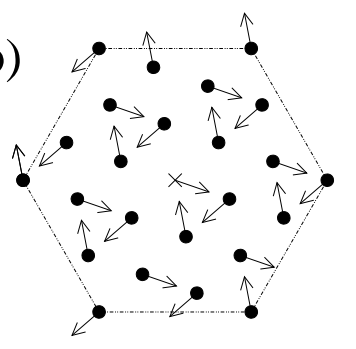

Figure 20. (a) Enumeration of intertrimer (intratrimer) nearest neighbours; (b) Classical $120^{\circ}$ Néel state with left chirality.

5.4.1 The quantum magnet Hamiltonian. The spinless interacting Fermi gas in the trimerised kagomé lattice is described by the following extended FermiHubbard Hamiltonian

$$
H_{\mathrm{FH}}=-\sum_{\langle a, b\rangle}\left(t_{a b} f_{a}^{\dagger} f_{b}+h . c .\right)+\sum_{\langle a, b\rangle} U_{a b} n_{a} n_{b},
$$

where $\langle a, b\rangle$ denotes nearest neighbors, $a=\{\alpha, i\}$ with $\alpha$ referring to intra-trimer indices and $i$ numbering the trimers. The $t_{a b}$ and $U_{a b}$ take the values $t$ and $U$ for intratrimer, and $t^{\prime}$ and $U^{\prime}$ for intertrimer couplings, $n_{a}=f_{a}^{\dagger} f_{a}$ and $f_{a}$ is the fermionic annihilation operator. The sites in each trimer are enumerated as in Fig. 20(a). We denote the 3 different intra-trimer modes by $f^{(i)}=\left(f_{1, i}+f_{2, i}+f_{3, i}\right) / \sqrt{3}$ (zero momentum mode), and $f_{ \pm}^{(i)}=\left(f_{1, i}+z_{ \pm} f_{2, i}+z_{ \pm}^{2} f_{3, i}\right) / \sqrt{3}$ (left and right chirality modes $)$, where $z_{ \pm}=\exp ( \pm 2 \pi i / 3)$.

In the limit of weak coupling between trimers, the extended Hubbard Hamiltonian for the problem of two fermions per trimer becomes equivalent to a quantum magnet on a triangular lattice with couplings that depend on the bond directions $[63,110$, $111]$

$$
H_{\text {magnet }}=\frac{J}{2} \sum_{i=1}^{N} \sum_{j=1}^{6} \sigma_{i}\left(\phi_{i \rightarrow j}\right) \sigma_{j}\left(\tilde{\phi}_{j \rightarrow i}\right),
$$

where $N$ denotes number of trimers, $J=4 U^{\prime} / 9$, and the nearest neighbours are enumerated as in Fig. 20(a). In Eq. (75) we have $\sigma_{i}(\phi)=\cos (\phi) \sigma_{x}^{(i)}+\sin (\phi) \sigma_{y}^{(i)}$, where the spin-1/2 operators are defined as: $\sigma_{x}^{(i)}=\left(f_{+}^{(i) \dagger} f_{-}^{(i)}+f_{-}^{(i) \dagger} f_{+}^{(i)}\right) / 2, \sigma_{y}^{(i)}=$ $-i\left(f_{+}^{(i) \dagger} f_{-}^{(i)}-f_{-}^{(i) \dagger} f_{+}^{(i)}\right) / 2$. The angles $\phi$ are: $\phi_{i \rightarrow 1}=\phi_{i \rightarrow 6}=0, \phi_{i \rightarrow 2}=\phi_{i \rightarrow 3}=2 \pi / 3$, $\phi_{i \rightarrow 4}=\phi_{i \rightarrow 5}=-2 \pi / 3, \tilde{\phi}_{1 \rightarrow i}=\tilde{\phi}_{2 \rightarrow i}=-2 \pi / 3, \tilde{\phi}_{3 \rightarrow i}=\tilde{\phi}_{4 \rightarrow i}=0, \tilde{\phi}_{5 \rightarrow i}=$ $\tilde{\phi}_{6 \rightarrow i}=2 \pi / 3$. The physical picture behind mapping the Fermi-Hubbard onto spin model is the following. There are two fermions in each trimer. In the ground state configuration one of them occupies the zero momentum mode, while the second one has to choose between either right or left chirality modes, so it stays in the superposition of these two modes that are further identified as spin- $1 / 2$ states.

5.4.2 Classical analysis. We first discuss the classical theory of the model (Eq. (75)), i.e., the large $S$ (spin) limit. In addition to being translationally invariant, 




Figure 21. (a) Classical $120^{\circ}$ Néel state with right chirality. Dots and triangles show $\sigma_{x}^{(i)} \sigma_{x}^{(10)}+\sigma_{y}^{(i)} \sigma_{y}^{(10)}$, where $|\vec{\sigma}|=1 / 2$ and the central spin defines the $\mathrm{x}$ axis; (b) Spin-spin correlations: $\left\langle\sigma_{x}^{(i)} \sigma_{x}^{(10)}+\sigma_{y}^{(i)} \sigma_{y}^{(10)}\right\rangle$. The upper (lower) set of values corresponds to $k T=0$ $\left(k T=10^{-2} J / 2\right)$. In both plots $N=21$ and $i=10$ at the central site.

the model (Eq.(75)) is invariant under the point group $Z_{6}=Z_{3} \cdot Z_{2}$. The generator of $Z_{3}$ is the combined rotation of the lattice by the angle $4 \pi / 3$, and of the spins by the angle $2 \pi / 3$, while the generator of $Z_{2}$ is the spin inversion in the lattice plane. There exist three ordered classical states with small unit cells that are compatible with this point-group symmetry of the model: a ferromagnetic state and two $120^{\circ}$ Néel type structures with left (Fig. 20(b)) and right (Fig. 21(a)) chiralities formally defined in $[110,111]$. The energies per site of these states are (i) $-3 S^{2} J / 4$ for ferromagnetic and right-handed Néel states; (ii) $3 S^{2} J / 2$ for left-handed Néel state. Hence, for $J<0$ the state with left-handed chirality will be the ground state. For $J>0$ the situation is more complicated since the state with right-handed chirality and the ferromagnetic state are degenerate ground states.

The analysis of classical ground states can be supplemented by a numerical study of the 12-spin cell done by fixing the direction of every spin to $n \pi / 3(n=0 \cdots 5)$, and checking the energies of the resulting $6^{12}$ configurations. This analysis has revealed that for $J<0$ there are 6 ground states $\left(Z_{6}\right.$ symmetry of $\left.(75)\right)$ each of them exhibiting the left chirality Néel order. For $J>0$ the results are dramatically different: there are 240 degenerate classical ground states in this case. Among them 6 right chirality Néel states and 6 ferromagnetic states. As will be seen below, the large number of degenerate classical ground states for $J>0$ finds its analogue in a large density of low-lying excitations of the quantum version of (Eq.(75)).

5.4.3 Quantum mechanical results. The insight into quantum mechanical properties of the system can be obtained through exact diagonalization of the Hamiltonian (Eq.(75)).

Numerical findings are presented in Fig. 21(b) and Tables 1 and 2. For $J>0$ the ground state exhibits the $120^{\circ}$ Néel order with right chirality. This is illustrated in Fig. 21(b), where the planar spin-spin correlations are presented. Direct comparison with the correlations of the classical state, Fig. 21(a), shows that the exact quantum correlations, although smaller, have the same order of magnitude and sign as the classical ones. Especially, the relative values of correlations compare nicely to the 


\begin{tabular}{cccccc}
\hline \hline & 1 & $\sqrt{3}$ & 2 & $\sqrt{7}$ & 3 \\
\hline $120^{\circ}$ & -0.125 & 0.25 & -0.125 & -0.125 & 0.25 \\
$\mathrm{~N}=24$ & -0.096 & 0.162 & -0.083 & -0.080 & 0.156 \\
$\mathrm{~N}=21$ & -0.085 & 0.135 & -0.071 & -0.067 & \\
\hline \hline
\end{tabular}

Table 1. Spin-spin correlations, $\left\langle\sigma_{x}^{(i)} \sigma_{x}^{(j)}+\sigma_{y}^{(i)} \sigma_{y}^{(j)}\right\rangle$, for $J>0$ as a function of distance (expressed in lattice units) between sites $i$ and $j$. The first row presents classical predictions for the $120^{\circ}$ Néel state (compare to Fig. 21).

\begin{tabular}{ccccc}
\hline \hline & 1 & $\sqrt{3}$ & 2 & $\sqrt{7}$ \\
\hline $120^{\circ}$ & -0.125 & 0.25 & -0.125 & -0.125 \\
$\mathrm{~N}=21$ & -0.134 & 0.237 & -0.117 & -0.116 \\
$\mathrm{~N}=12$ & -0.137 & 0.251 & -0.125 & \\
\hline \hline
\end{tabular}

Table 2. The same as in Table 1 but for $J<0$.

classical result. Interestingly, the $120^{\circ}$ Néel order survives at finite temperatures, as is indicated by the results obtained for $k T=10^{-2} \mathrm{~J} / 2$ (Fig. 21(b)). At such temperatures about 800 low energy eigenstates contribute to the correlations. To quantify how exceptionally dense the excitation spectrum is, we note that for $N=$ 21 there are about 2000 (800) excited states with energies less than $0.09 \mathrm{~J}(0.05 \mathrm{~J})$ above the ground state. Most of them support the spin order of the ground state so that antiferromagnetic order persists at finite temperatures. Moreover, numerical simulations show that the spectrum becomes more dense as $N$ increases.

The exact diagonalizations do not give a definite answer of whether the gap vanishes in the limit of infinite lattice. What can be found out from exact diagonalizations is that the gap, if any, should be smaller than about $10^{-2} \mathrm{~J} / 2$. The appearance of this very small energy scale is surprising. The smallness of the gap and the large density of low-energy states resemble very much the behaviour of a quantum spin liquid of type II. The spin liquids of type II, however, possess extremely short range correlations, which is in striking opposition to the behaviour of the considered quantum magnet. For these reasons it was proposed to name this system a quantum spin-liquid crystal.

The above results for $J>0$ are in a strong contrast to those for $J<0$. In the latter case the ground state is the standard quantum antiferromagnet with $120^{\circ}$ Néel order and left chirality. The spectrum is gapped, and the classical spin-spin correlations approximate well the quantum ones (Table 2). In fact, the semiclassical theory works remarkably well even for system sizes as small as $N=12$. The gap is of the order of $|J| / 2$ in this case, so that there are at most few states with energies substantially below $|J| / 2$ for $J<0$, as opposed to the huge number for $J>0$.

As it is evidenced from the above discussion, the theoretical studies of the quantum spin liquid crystals are limited to relatively small systems. We regard future experiments as the best possible verification of these predictions. 


\subsection{Realization of frustrated models in cold atom/ion systems}

Here we focus on control of interactions and recent proposals based on ultracold bosonic or fermionic atoms [64], cold gases of polar molecules [115], and trapped ions [495-497]. Realizations of frustrated models in ultracold atomic systems requires either the creation of a lattice with appropriate geometry, or the control of the effective atomic interactions. Geometry of the lattice, as we already mentioned, can be engineered using superlattice techniques (see Fig. 22 and Ref. [111] for the details in the case of kagomé lattices).

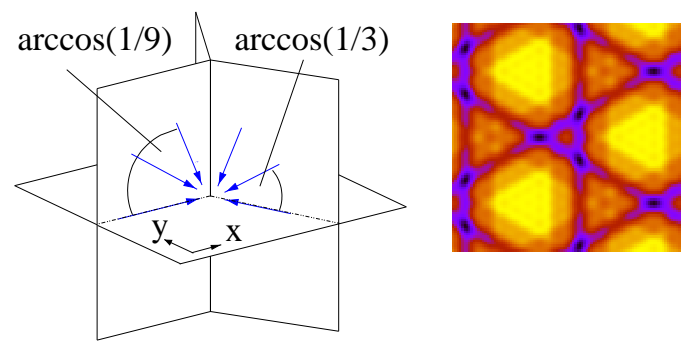

Figure 22. Scheme of the proposed experimental set-up. Each arrow depicts a wave vector of a standing wave laser. The three vertical planes intersect at an angle of $120^{\circ}$. Dark (dark blue in the online version) spots in the right kagomé figure indicate the potential lattice minima (from [111]).

5.5.1 Simulators of spin systems with topological order. We discuss here the proposal of Duan, Demler and Lukin, for realization of quantum magnets in a system of cold atoms placed in an optical lattice [64]. For simplicity, we assume that the lattice is filled with bosons. It is made of standing laser beams producing the periodic potential $V_{\mu \sigma} \sin ^{2}\left(\vec{k}_{\mu} \cdot \vec{r}\right)$ in the direction $\mu(\vec{k}$ is the beam wave vector $)$. The index $\sigma=\{\uparrow, \downarrow\}$ accounts for the fact that bosons are trapped in two different internal states by independent lattice potentials.

The system is governed by the following Hubbard Hamiltonian

$$
H_{\text {atoms }}=-\sum_{\langle i j\rangle \sigma}\left(t_{\mu \sigma} b_{i \sigma}^{\dagger} b_{j \sigma}+\text { h.c. }\right)+\frac{1}{2} \sum_{i \sigma} U_{\sigma} n_{i \sigma}\left(n_{i \sigma}-1\right)+U_{\uparrow \downarrow} \sum_{i} n_{i \uparrow} n_{i \downarrow},
$$

where $\langle i j\rangle$ denotes nearest neighbors, $b_{i \sigma}\left(b_{i \sigma}^{\dagger}\right)$ is the annihilation (creation) operator of bosons in the $i$-th lattice site. The Hamiltonian (Eq.(76)), describes tunneling of bosons in different internal states between neighbouring lattice sites, and their on-site interactions.

The Hamiltonian (Eq.(76)) can be cast into the quantum magnet form if the following conditions are satisfied (i) $t_{\mu \sigma} \ll U_{\sigma}, U_{\uparrow \downarrow}$, which typically requires large enough $V_{\mu \sigma}$; (ii) $\left\langle n_{i \uparrow}\right\rangle+\left\langle n_{i \downarrow}\right\rangle \simeq 1$. Then, in the lowest order nontrivial approximation in 
$t_{\mu \sigma} / U_{\uparrow \downarrow}$, one gets

$$
H_{a t o m s} \approx \sum_{\langle i, j\rangle}\left[\lambda_{\mu z} \sigma_{i}^{z} \sigma_{j}^{z}-\lambda_{\mu \perp}\left(\sigma_{i}^{x} \sigma_{j}^{x}+\sigma_{i}^{y} \sigma_{j}^{y}\right)\right],
$$

where $\sigma_{i}^{z}=n_{i \uparrow}-n_{i \downarrow}, \sigma_{i}^{x}=b_{i \uparrow}^{\dagger} b_{i \downarrow}+b_{i \downarrow}^{\dagger} b_{i \uparrow}$, and $\sigma_{i}^{y}=-i\left(b_{i \uparrow}^{\dagger} b_{i \downarrow}-b_{i \downarrow}^{\dagger} b_{i \uparrow}\right)$, and

$$
\lambda_{\mu z}=\frac{t_{\mu \uparrow}^{2}+t_{\mu \downarrow}^{2}}{2 U_{\uparrow \downarrow}}-\frac{t_{\mu \uparrow}^{2}}{U_{\uparrow}}-\frac{t_{\mu \downarrow}^{2}}{U_{\downarrow}}, \quad \lambda_{\mu \perp}=\frac{t_{\mu \uparrow} t_{\mu \downarrow}}{U_{\uparrow \downarrow}} .
$$

The Hamiltonian (Eq.(77)) corresponds to anisotropic Heisenberg spin-1/2 model, whose different variations can be studied due to the possibility of adjustment of $t_{\mu \sigma}$, $U_{\sigma}$ and $U_{\uparrow \downarrow}$ couplings. This can be achieved, e.g., via manipulation of the lattice potentials imposed on atoms: $V_{\mu \sigma}$.

To illustrate the possibilities offered by cold atomic systems, let us consider the limit of $t_{\mu \downarrow} / t_{\mu \uparrow} \rightarrow 0$, i.e., $V_{\mu \uparrow} \ll V_{\mu \downarrow}$. Then, obviously the $H_{\text {atoms }}$ Hamiltonian approximates the nearest-neighbor Ising model (Eq.(66)). Depending on $V_{\mu \uparrow}$ the couplings $\lambda_{\mu z}$ can be either isotropic or anisotropic. Moreover, they can be all either positive or negative depending on the $U_{\uparrow \downarrow} / U_{\uparrow}$ ratio. Therefore, as discussed in Sec. 5.1 both ferromagnetic and antiferromagnetic Ising model can be simulated with cold bosons in a lattice. Additionally, as discussed in [64], the successful experimental realization of the Ising model in a system of cold atoms can be very useful in the experimental implementation of the one-way quantum computer, introduced by Raussendorf and Briegel [498,499].

Perhaps the most unusual quantum magnet, that can be realized using the ideas presented above, corresponds to the anisotropic $2 \mathrm{D}$ spin- $1 / 2$ model on a hexagonal lattice. A hexagonal lattice can be created in a carefully designed setup of laser beams [64]. Assuming the same as above for derivation of Eq.(77), and additionally $U_{\uparrow \downarrow} \approx U_{\uparrow} \approx U_{\downarrow} \approx U$ the Hamiltonian (Eq.(76)) can be transformed into

$$
H_{\text {atoms }} \approx \lambda_{x} \sum_{x-l i n k s} \sigma_{i}^{x} \sigma_{j}^{x}+\lambda_{y} \sum_{y-l i n k s} \sigma_{i}^{y} \sigma_{j}^{y}+\lambda_{z} \sum_{z-l i n k s} \sigma_{i}^{z} \sigma_{j}^{z},
$$

where the summation goes over nearest neighbours $i$ and $j$ placed on the $x, y, z$ links - see Fig. 19. The couplings read $\lambda_{\mu}=-t_{\mu+}^{2} /(2 U)$, where $t_{\mu+}$ is the rate of tunneling of the atom being in the eigenstate of the Pauli operator $\sigma_{\mu}$ with eigenvalue +1 .

The Hamiltonian (Eq.(78)) describes the Kitaev model [500] with topological order. Excitations in this model are both Abelian and non-Abelian anyons [501,502], having exotic fractional statistics. It is exactly solvable, and possesses other exciting features [500]. Its realization should provide an exceptional possibility for experimental observation of Abelian and non-Abelian anyons, and could serve for applications in quantum information as protected qubits.

5.5.2 Frustrated models with polar molecules. Micheli et al. [115] have proposed a scheme to realize spin Hamiltonians with polar molecules. In fact, recently various schemes have been proposed for trapping different states of cold 
polar molecules. New developments in this area can be found in Ref. [146] (for experiments, see [503]).

In Ref. [115], the authors start with two polar molecules, trapped in an optical lattice. The outermost shell of an electron of a heteronuclear molecule represents the spin- $1 / 2$ system. The total Hamiltonian of a pair of heteronuclear molecules trapped in an optical lattice is given by

$$
H=\underbrace{\left(H_{d d}+\sum_{i=1}^{2} H_{m}^{i}\right)}_{H_{\text {int }}}+\underbrace{\left(\sum_{i=1}^{2} \frac{P_{i}^{2}}{2 m}+V_{i}\left(x-\overline{x_{i}}\right)\right)}_{H_{\text {ext }}},
$$

where $H_{\text {int }}$ and $H_{\text {ext }}$ represent respectively the internal and external dynamics of molecules. $H_{d d}$ is the Hamiltonian for dipole-dipole interactions between two molecules. The rotational excitation of each molecule is described by the Hamiltonian

$$
H_{m}=B N^{2}+\gamma N \cdot S
$$

where $N$ is the dimensionless orbital angular momentum of the nuclei and $S$ is the electronic spin. Here $B$ is the rotational constant and $\gamma$ is the spin-rotation coupling constant. The rotational motion of molecules is coupled by the dipole-dipole interactions that are already present in the heteronuclear molecule. To obtain strong dipole-dipole interactions, a microwave field is introduced. If the field polarization is set to the $z$-axis of the two molecules, and the frequency is fixed as near resonant with the excited state potential, it leads to some spin pattern of ground states. By changing the frequency and field polarization, it is possible to obtain various spin models, e.g., Ising, Heisenberg, and Kitaev models, that have been discussed earlier (see Table I of [115]). The advantage of this model is the strong dipole-dipole interactions which is due to the inherent properties of these molecules, and is also due to the introduction of microwave fields. The couplings are strong relative to decoherence rates.

5.5.3 Ion-based quantum simulators of spin systems. It was recently proposed by Porras and Cirac that different spin systems can also be simulated with cold ions $[495,496]$. This protocol, if implemented experimentally, should allow for studies of different quantum magnets in ion traps. As a result, the ions could then be used for investigation of condensed matter problems. In fact, it seems that ions are perfectly suitable for such experimental studies since (i) they can be trapped and cooled very efficiently; (ii) the position and internal state of every single ion can be measured and manipulated almost at will; (iii) the external parameters of that system can be well controlled and changed in real time.

Below we will briefly present the Porras and Cirac proposal. The spin $1 / 2$ states are encoded in two internal hyperfine states of each ion. The local dynamics of these states is governed by the Hamiltonian

$$
H_{m}=\sum_{j=1 \ldots N}^{\alpha=x, y, z} B^{\alpha} \sigma_{j}^{\alpha},
$$


where $N$ is the number of ions, $B^{x}$ and $B^{y}$ are "analogs" of the magnetic field induced by lasers resonant with the internal transition, whereas $B^{z}$ is the energy gap between the two internal states.

The ions are affected by both an external harmonic trapping potential with frequencies $\left(\Omega_{x}, \Omega_{y}, \Omega_{z}\right)$ and Coulomb interactions, which is described by the potential:

$$
V=\frac{m}{2} \sum_{j=1 \ldots N}\left(\Omega_{x}^{2} x_{j}^{2}+\Omega_{y}^{2} y_{j}^{2}+\Omega_{z}^{2} z_{j}^{2}\right)+\sum_{j>i} \frac{e^{2}}{\sqrt{\left(x_{i}-x_{j}\right)^{2}+\left(y_{i}-y_{j}\right)^{2}+\left(z_{i}-z_{j}\right)^{2}}},
$$

where $m$ is the mass of the ion and $e$ is the electron charge. It is further assumed that the trap has a cigar-shaped geometry with axial $z$ direction and radial $x$ and $y$ ones: $\Omega_{z} \ll \Omega_{x}, \Omega_{y}$. The competition between harmonic squeezing and Coulomb repulsion results in oscillations of ions around equilibrium positions. Dynamics of these oscillations is governed by the vibrational Hamiltonian: $H_{v}=\sum_{j=1 \ldots N}^{\alpha=x, y, z} \frac{p_{j}^{\alpha 2}}{2 m}+$ $V$, where $p_{j}^{\alpha}$ are momentum operators of the $j$-th ion. After expansion of $V$ up to a quadratic order in ions displacement from equilibrium positions, the vibrational Hamiltonian can be diagonalized in a standard way in terms of collective modes (phonons):

$$
H_{v}=\sum_{n}^{\alpha=x, y, z} \hbar \omega_{\alpha, n} a_{\alpha, n}^{\dagger} a_{\alpha, n},
$$

where $a_{\alpha, n}$ and $a_{\alpha, n}^{\dagger}$ are the phonon annihilation and creation operators and $\omega_{\alpha, n}$ are the collective mode frequencies.

Finally, one needs a coupling between $H_{m}$ and $H_{v}$, i.e., between "effective" spins and phonons. This is achieved by placing the ions in off-resonant standing wave beams. For instance, imposing standing light waves on ions one can realize the following Hamiltonian [496]:

$$
H_{f}=-F_{x} \sum_{j} x_{j}|\uparrow\rangle\left\langle\left.\uparrow\right|_{z, j}-F_{y} \sum_{j} y_{j} \mid \uparrow\right\rangle\left\langle\left.\uparrow\right|_{y, j},\right.
$$

where $\sigma^{\alpha}|\uparrow\rangle_{\alpha}=|\uparrow\rangle_{\alpha}$. This Hamiltonian assumes that the laser beams push ions in the upper state only, which can be achieved by a proper adjustment of the relative phases of the beams.

The total Hamiltonian of the system becomes then $H=H_{m}+H_{v}+H_{f}$. The final step to get the clear picture of the phonon-mediated interactions between "effective" spins relies on the unitary transformation $H \rightarrow U H U^{\dagger}$ with $U$ specified in $[495,496,504]$. The results of this transformation will be illustrated on particular examples.

Assuming that $F_{x} \neq 0$ and $F_{y}=0$, one gets to the lowest order in $\eta_{\alpha}=$ $F_{\alpha} \sqrt{\hbar / 2 m \Omega_{\alpha}} / \hbar \Omega_{\alpha}$

$$
H \approx \frac{1}{2} \sum_{i, j} J_{i, j}^{[x]} \sigma_{i}^{z} \sigma_{j}^{z}+\sum_{i} B^{x} \sigma_{i}^{x}+H_{v}+H_{E},
$$

where

$$
J_{i, j}^{[\alpha]} \sim \frac{1}{\left|\left\langle z_{i}\right\rangle-\left\langle z_{j}\right\rangle\right|^{3}}
$$


with $\left\langle z_{i}\right\rangle$ being the equilibrium position of the $i$-th ion. The first two terms in Eq.(81) act in the "spin" space and exhibit the quantum Ising model type of spinspin and spin-field interactions. The fact that the interactions are long-range makes quantum properties even richer then those of a standard Ising model with only nearest neighbor terms. Therefore, one gets the possibility of studying the fascinating Ising-type model [496] in an ion chain if the corrections coming from the perturbation $H_{E}[495,496]$ are negligible. To quantify influence of the perturbation $H_{E}$ on spin dynamics, we note that the deviations of expectation values of $M$-spin(site) observables, induced by skipping the $H_{E}$ term, scale as $M \eta_{\alpha}^{2}$. Since the observables of interest during analysis of quantum phase transitions correspond usually to $M=1$ or 2 classes, the corrections coming from $H_{E}$ should not cause major problems.

Another possibility shows up when there are two forces acting in radial directions $x$ and $y\left(F_{x}, F_{y} \neq 0\right)$. Then one gets that the $U$ transformed Hamiltonian is

$$
H \approx \frac{1}{2} \sum_{i, j}\left(J_{i, j}^{[x]} \sigma_{i}^{z} \sigma_{j}^{z}+J_{i, j}^{[y]} \sigma_{i}^{y} \sigma_{j}^{y}\right)+\sum_{i} B^{x} \sigma_{i}^{x}+H_{v}+H_{E},
$$

where $J_{i, j}^{[\alpha]}$ are given by Eq.(82). This time, the first two terms correspond to the $\mathrm{XY}$ model with long-range interactions. The perturbation affecting spin dynamics, $H_{E}$, is now a little different than the one above, but as long as $\omega_{x} \neq \omega_{y}$ the errors induced by leaving the $H_{E}$ out are of the same order. Hence, we conclude that ion chains can be used for simulation of yet another important spin model.

Though the above discussion was focused on the one-dimensional geometry of the ion trap, a similar approach can be used for studies of ions in two-dimensional geometries, where they form Coulomb crystals $[505,506]$. This time, two dimensional "effective" spin models should show up. Their experimental realization would greatly facilitate studies of frustrated quantum magnets.

Finally, we remark that the scheme discussed in this section offers an access to measurements and manipulations of arbitrarily selected ion(s): an exciting opportunity unavailable in traditional condensed matter systems. Interestingly, since the "spin" configuration is encoded in ions internal states, the fluorescence signal of the sample can contain enough information for detection of different phases.

\section{$6 \quad$ Ultracold spinor atomic gases}

\subsection{Introduction}

Interactions in bosonic systems with spin degrees of freedom host a wide variety of exotic phases at zero temperature and a dynamics clearly differentiated from the one displayed by scalar gases. Spin effects are enhanced in the limit of small occupation number and strong interactions. This fact, makes the study of ultracold spinors in optical lattices of primordial importance and a very valuable tool to deepen our understanding of magnetic ordering and condensed matter related issues.

On the other hand, there exists also an increasing interest in the study of spinor 
fermions in optical lattices since these systems could serve as quantum simulators of fermionic Hubbard model and shed some light on the problem of high Tc-superconductivity. we shall address this point at the end of the chapter.

The spinor degree of freedom on alkaline gases corresponds to the manifold of degenerate (in the absence of an external magnetic field) Zeeman hyperfine energy levels. The energy levels are described by the total angular momentum $\mathbf{F}=\mathbf{I}+\mathbf{J}$, where $\mathbf{I}$ refers to the nuclear spin and $\mathbf{J}=\mathbf{L}+\mathbf{S}$ describes the total electronic angular momentum. In the ground state, alkaline atoms have a single electron in an $n s$ orbital, their electronic angular momentum $J=S=1 / 2$ and, therefore, they have two possible hyperfine of Zeeman states. The nuclear spin I depends on each atomic species. ${ }^{133} \mathrm{Cs}$ atoms have a nuclear spin $I=7 / 2$, while ${ }^{85} \mathrm{Rb}$ atoms have $I=5 / 2$, and ${ }^{87} \mathrm{Rb}$ and ${ }^{23} \mathrm{Na}$ have $I=3 / 2$. In the case of Cesium, the hyperfine manifolds correspond to $F=4,3$; for ${ }^{85} \mathrm{Rb}$ they correspond to $F=3,2$ and ${ }^{87} \mathrm{Rb}$ and ${ }^{23} \mathrm{Na}$ have manifolds $F=2,1$. Each ground state manifold consists of all Zeeman states associated to a given total angular momentum, i.e. $\left\{\left|F, m_{F}\right\rangle\right\}$ where $m_{F}=-F, \ldots, F$. In this context, we identify the total angular momentum of the atom $F$ as the atomic spin.

If atoms are magnetically trapped, the degeneracy is broken and the atoms minimize their energy in the so-called "weak field" seeking states, characterised by a fixed hyperfine level $\left|F, m_{F}\right\rangle$. The simultaneous confining of different "low field seeking" states is usually unstable against spin-spin collisions. This is why magnetic trapping results in a frozen atomic spin " $F$ " and spin projection " $m_{F}$ " [508]. In that case, the bosonic quantum field operator $\Psi$ describing the creation of a boson in position $\mathbf{r}$ is a scalar in the spinor space with no dependence whatsoever on the hyperfine magnetic level. In turn, the mean field description of the ultracold bosonic gas is done via a scalar order parameter which has not explicit dependence on the ground state manifold $\left|F, m_{F}\right\rangle$ in which the atoms were trapped. In some cases, despite the fact that the levels are not anymore degenerate, it is possible to magnetically trap simultaneously more than one hyperfine component. This is the case of ${ }^{87} \mathrm{Rb}$, where usually the singlet and the triplet scattering lengths are practically equal and thus spin exchange collisions are highly suppressed. It is then possible to magnetically trap simultaneously the $\left|F=2, m_{F}=2\right\rangle$ and $\left|F=1, m_{F}=-1\right\rangle$ states. In such cases, one speaks of "multi-component" ultracold gases, but still the spin of the atoms remains "frozen", although with two different possibilities [507]. On the contrary, if atoms are optically trapped with a far-off resonance laser, then all atoms, regardless of their hyperfine level are simultaneously trapped. The bosonic quantum field operator in such case is no longer an scalar and has to incorporate this new degree of freedom $\Psi_{m_{F}}$. In turn, the corresponding order parameter describing mean field approach, becomes a vector whose components correspond to the different accessible hyperfine levels [508,509] and which transforms under rotations in the spin space as a vector preserving the symmetries present in the corresponding spin space.

\subsection{Spinor interactions}

Like in the scalar case, ultracold atomic spinor interactions can be parametrised by two-body short range (s-wave) collisions. In the most general scenario, symmetry arguments impose that the collisions between two identical bosons in a hyperfine 
spin level $\left|F, m_{F}\right\rangle$ are restricted to total spin $S=2 F, 2 F-2, \ldots, 0$. The contact potential can be written as:

$$
V=\sum_{S=0,2, \ldots, 2 F} g_{S} P_{S}
$$

where $P_{S}(S=0,2, \ldots, 2 F)$ denotes the projector onto the subspace with total spin $S$. The interaction strength $g_{S}$ characterising the contact potential interaction of the different $S$ channels are given by $g_{S}=\frac{4 \pi \hbar^{2} a_{S}}{m}$, where $a_{S}$ is the corresponding scattering length and $m$ the atomic mass. The different values of the various $a_{S}$ will lead to distinct magnetic ordering.

To understand the ground state properties as well as the dynamics, it is convenient to express the interaction potential $V$ in terms of spin operators by using different operator identities, e.g. $I=\sum_{S=0,2, \ldots, 2 F} P_{S}$ and $\mathbf{F}_{1} \cdot \mathbf{F}_{2}=\sum \lambda_{S} P_{S}$ where $\lambda_{S}=$ $(1 / 2)[S(S+1)-F(F+1)]$. For spin $F=1$, the total spin is $S=2,0$ and using the above identities the contact potential can be written as:

$$
V_{(F=1)}=c_{0}+c_{2} \mathbf{F}_{1} \cdot \mathbf{F}_{2}
$$

The terms with coefficients $c_{0}$ and $c_{2}$ describe spin-independent and spin-dependent binary elastic collisions respectively in the combined symmetric channels of total spin 0 and 2, and are expressed in terms of the s-wave scattering lengths $a_{0}$ and $a_{2}$ as: $c_{0}=4 \pi \hbar^{2}\left(a_{0}+2 a_{2}\right) / 3 m$ and $c_{2}=4 \pi \hbar^{2}\left(a_{2}-a_{0}\right) / 3 m[509,510]$.

It is straightforward to generalize some spin identities to larger spins [509] by noting that $\left(\boldsymbol{F}_{1} \cdot \boldsymbol{F}_{2}\right)^{n}=\sum \lambda_{S}^{n} P_{S}$. Then the interaction potential can be rewritten as $V=\sum_{n=0}^{2 F} c_{n}\left(\mathbf{F}_{1} \cdot \mathbf{F}_{2}\right)^{n}$ where the $c_{n}$ are linear combinations of the different scattering lengths $a_{S}$.

For $F=2$, there are 3 possible channels with total spin $S=0,2,4$. The contact potential can be expressed as

$$
V_{(F=2)}=c_{0}+c_{1} P_{0}+c_{2} \mathbf{F}_{1} \cdot \mathbf{F}_{2},
$$

where the coefficients are given by $c_{0}=4 \pi \hbar^{2}\left(3 a_{4}+4 a_{2}\right) / 7 m$ and $c_{1}=4 \pi \hbar^{2}\left(3 a_{2}-\right.$ $\left.10 a_{2}+7 a_{0}\right) / 7 m$ and $c_{2}=4 \pi \hbar^{2}\left(a_{4}-a_{2}\right) / 7 m$. The projector $P_{0}$ can be further expressed in terms of "singlet" pair operator as we shall see later.

\section{3 $F=1$ and $F=2$ spinor gases: Mean field regime}

The experimental achievement of Stenger et al. [511] in trapping ${ }^{23} \mathrm{Na}$ by optical means in 1998, triggered the study of spinor ultracold gases. A mean field approach to describe a $F=1$ condensate was developed by Ho [509], and independently by Ohmi and Mashida [512] in the same year. Koashi and Ueda [510], and Ciobanu, Yip, and Ho [514] have calculated the mean field phase diagram of $F=2$ spinor condensates. More recently, Ueda and Koashi [515] have studied mean field theory for $F=2$ atoms in presence also of a magnetic external field. The recent success on condensing Chromium atoms [145] have also initiated the study of mean field phases for spin $F=3[516,517]$. Recent experiments concern studies of dynamics of spinor BECs in traps [518-523] (for the theory, see [288]), and in lattices [524]. 
In scalar bosonic weakly interacting systems, where a mean field approach can be used, the ground state is found by approximating the bosonic operator of the corresponding Hamiltonian by its mean, and minimizing the energy functional under the constrain that the number of particles is fixed (grand canonical ensemble) $\partial\langle H-\mu N\rangle / \partial \mu=0$. For spinor gases, one follows the same approach [509] and minimization of the energy leads to different ground states depending on the values of the spin-spin coupling.

The Hamiltonian of a trapped cloud of ultracold atoms with spin $F=1$ in second quantization reads

$$
\begin{aligned}
H= & \int d^{3} r\left\{\Psi_{m}^{\dagger}\left(-\frac{\hbar^{2}}{2 M} \nabla^{2}+V_{e x t}\right) \Psi_{m}\right. \\
& \left.+\frac{c_{0}}{2} \Psi_{m}^{\dagger} \Psi_{j}^{\dagger} \Psi_{j} \Psi_{m}+\frac{c_{2}}{2} \Psi_{m}^{\dagger} \Psi_{j}^{\dagger} \mathbf{F}_{m k} \cdot \mathbf{F}_{j l} \Psi_{l} \Psi_{k}\right\}
\end{aligned}
$$

where $\Psi_{m}(\mathbf{r})\left(\Psi_{m}^{\dagger}\right)$ is the field operator that annihilates (creates) an atom in the $m$-th hyperfine state $\left|F=1, m_{F}\right\rangle$ at point $\mathbf{r}$. The external trapping potential, $V_{\text {ext }}$, is normally assumed to be spin-independent.

There are two distinct ground state mean field phases for $\operatorname{spin} F=1$ :

- Ferromagnetic phase for $F=1$.

The system presents ferromagnetic order if $c_{2}<0$ (i.e. $g_{0}>g_{2}$ ). This configuration minimizes the energy by imposing that $\langle F\rangle^{2}=1$. For a spin pointing along $\vec{n}=(\cos \alpha \sin \beta, \sin \alpha \sin \beta, \cos \beta)$ the condensate order parameter is given by:

$$
\xi=e^{i \varphi} U(\alpha, \beta)\left(\begin{array}{l}
1 \\
0 \\
0
\end{array}\right)=e^{i \varphi}\left(\begin{array}{c}
e^{-i \alpha} \cos ^{2}(\beta / 2) \\
\sqrt{(2)} \cos (\beta / 2) \sin (\beta / 2) \\
e^{i \alpha} \sin ^{2}(\beta / 2)
\end{array}\right) .
$$

This is, for example, the ground state of ${ }^{87} \mathrm{Rb}$ in the $F=1$ manifold.

- Polar phase for $F=1$.

The system presents polar (sometimes called antiferromagnetic) ordering if $c_{2}>$ 0 . This is the case for ${ }^{23} \mathrm{Na}$ or ${ }^{85} \mathrm{Rb}$. Minimisation is achieved by demanding that the expectation values of the spin component are zero along any direction, i.e. $\langle F\rangle=0$. However, variances are not equal to zero, indicating that the system does not posses rotational invariance. The general expression for the spinor in this case reads

$$
\xi=e^{i \varphi}\left(\begin{array}{l}
0 \\
1 \\
0
\end{array}\right)=e^{i \varphi}\left(\begin{array}{c}
-e^{-i \alpha} \sin (\beta) / \sqrt{(2)} \\
\cos (\beta) \\
e^{i \alpha} \sin (\beta) \sqrt{(2)}
\end{array}\right) .
$$

Notice that this "antiferromagnetic" ordering do not refer to orient the spins antiparallely as it happens classically.

For $F=2$ (for experiments see [519]) collisions can occur in one more channel corresponding to total spin $S=4$. As a result there is one more possible magnetic ground state, the so-called cyclic ground state. Now, to characterise the different ground states one should consider not only magnetization, i.e. $\langle F\rangle$, but also the "spin singlet pair creation" expectation value $[514,515]$. The term proportional to $P_{0}$ in the contact potential (see Eq.(86)) can be expressed by means of a "spin 
singlet pair operator" as $P_{0}=(2 / 5) \bar{S}_{+} \bar{S}_{-}$, where $\bar{S}_{+}=a_{0}^{\dagger} a_{0}^{\dagger} / 2-a_{1}^{\dagger} a_{-1}^{\dagger}+a_{2}^{\dagger} a_{-2}^{\dagger}$, and $a_{\sigma}^{\dagger}\left(a_{\sigma}\right)$ creates (annihilates) a particle with spin projection $\sigma\left(\bar{S}_{-}=\bar{S}_{+}^{\dagger}\right)$. The operator $\bar{S}_{-}$applied on the vacuum creates, except for normalization, two bosons in a spin singlet state. Ground states can be classified according to their expectation values of the "magnetisation" and the "singlet pair creation":

$$
\begin{aligned}
\langle F\rangle & =\sum_{m=-2, n=-2}^{m=2, n=2} F_{m n} \xi_{m}^{*} \xi_{n} \\
\left\langle\bar{S}_{+}\right\rangle & =\frac{1}{2} \sum_{m}(-1)^{m} \xi_{m} \xi_{-m} .
\end{aligned}
$$

- Ferromagnetic phase for $F=2$.

The ferromagnetic phase is achieved for $\langle F\rangle \neq 0$ and $\left\langle\bar{S}_{+}\right\rangle=0$. The system prefers ferromagnetic order if $c_{1}<0$ and $c_{1}-\left(c_{2} / 20\right)<0$. The expectation value of the magnetisation can either be $\langle F\rangle=2$ or $\langle F\rangle=1$ but for both cases $\left\langle\bar{S}_{+}\right\rangle=0$. A representative of maximal spin projection, ground state is given by:

$$
\xi=e^{i \varphi}\left(\begin{array}{l}
1 \\
0 \\
0 \\
0 \\
0
\end{array}\right) .
$$

This corresponds, for instance, to the ground state $F=2$ of ${ }^{87} \mathrm{Rb}$.

- Polar condensate for $F=2$.

Polar (antiferromagnetic) ordering is described by a non zero singlet amplitude $\left\langle\bar{S}_{+}\right\rangle \neq 0$ and zero magnetisation $\langle F\rangle=0$. A representative of the corresponding spinor order parameter is given by:

$$
\xi=e^{i \varphi}\left(\begin{array}{l}
0 \\
0 \\
1 \\
0 \\
0
\end{array}\right) .
$$

The phase space boundaries for polar phase are given by $c_{2}<0$ and $c_{1}-\left(c_{2} / 20\right)>$ 0 .

- Cyclic phase for $F=2$.

Finally, cyclic ordering appears for $c_{1}, c_{2}>0$ there $\langle F\rangle=0$ and $\left\langle\bar{S}_{+}\right\rangle=0$.

6.3.1 $F=1$ gases in optical lattices. Studies of $F=1$ systems have already been carried out by Demler's group $[118,119]$. They have derived an approximate phase diagram in the case of antiferromagnetic interactions. As in the standard Bose-Hubbard model, an $F=1$ spinor gas undergoes superfluid to Mott insulator transition as tunneling is decreased. In the antiferromagnetic case in $2 \mathrm{D}$ and $3 \mathrm{D}$, the SF phase is polar, and so are the Mott states with an odd number $N$ of atoms per site (those states are also termed nematic). In the case of even $N$, for small 
tunneling the Mott states are singlets, and for moderate tunneling there occurs a first order transition to the nematic state.

In $1 \mathrm{D}$ there is furthermore the possibility of a dimerized valence bond solid state, as in the Majumdar-Ghosh model [60] (discussed also in subsection 5.2.2). This possibility was studied by Yip [120], who derived an effective spin Hamiltonian for the MI state with $N=1$, and used the variational ansatz interpolating between dimer and nematic states to argue that in a wide range of parameters the spinor ${ }^{23} \mathrm{Na}$ lattice gas should have the dimer ground state in $1 \mathrm{D}, 2 \mathrm{D}$, and $3 \mathrm{D}$. This is a very interesting result, since dimer states have not been so far observed in experiments. This result has been supported by rigorous studies in Ref. [121] for spin systems with an even number of spins described by the same effective Hamiltonian: it was shown that while the ground state of the system has total spin $S_{t o t}=0$, the first excited state has $S_{t o t}=2$. Yip's results were recently confirmed by Rizzi et al. [525], who numerically studied the SF - MI transition in the $F=1$ BoseHubbard model in 1D, and found that the system is always in the dimerized state in low tunneling regime of the first MI lobe, where the effective spin model of Ref. [118, 119] works. Similar results were obtained by Porras et al. [526]. Thus, nematic order seems to be strictly speaking absent in 1D in the thermodynamic limit. However, susceptibility to nematic ordering grows close to the border of the ferromagnetic phases, indicating that it may persist in finite systems. A completely new insight can be gained by looking at entanglement transport properties of $F=1$ chains [527], which seem to confirm existence of nematic and trimer regions for finite systems.

Another interesting aspect, namely the possibility of controlling the order of the SF - MI transition by using appropriately polarized (lin- $\theta$-lin) laser fields to form the optical lattice was investigated in Refs. [528,529]. Such a laser configuration couples the states with $m_{F}= \pm 1$, so that the system becomes effectively two-component.

6.3.2 Bose-Hubbard model for spin 1 particles. The derivation of the Bose-Hubbard Hamiltonian for ultracold spinor gases is performed in the same way as in the scalar case. One has to add to the scalar Bose-Hubbard model the spin dependent part of the interaction. Following the identities given in the previous part of the section, the Bose-Hubbard Hamiltonian for spin 1 particles is obtained straightforwardly [119]:

$$
\begin{aligned}
H= & -t \sum_{<i j>, \sigma}\left(b_{\sigma i}^{\dagger} b_{\sigma j}+b_{\sigma j}^{\dagger} b_{\sigma i}\right)+\frac{c_{0}}{2} \sum_{i} n_{i}\left(n_{i}-1\right) \\
& +\frac{c_{2}}{2} \sum_{i}\left(\vec{F}_{i}^{2}-2 n_{i}\right)-\mu \sum_{i} n_{i}
\end{aligned}
$$

where $b_{\sigma i}$ annihilates a boson in a hyperfine state $m_{F}=\sigma$ at site $i, n_{i}$ denotes the number of particles at site $i$ and $\mathbf{F}_{i}=\sum_{\sigma \sigma^{\prime}} b_{\sigma i}^{\dagger} \boldsymbol{T}_{\sigma \sigma^{\prime}} b_{\sigma^{\prime} i}$ is the spin operator at site $i$ ( $\boldsymbol{T}_{\sigma \sigma^{\prime}}$ being the usual spin matrices for a spin-1 particle) and $\langle i j>$ denotes pairs of nearest neighbours in the lattice. The first two terms in the Hamiltonian represent tunneling between nearest-neighbor sites and Hubbard interactions between bosons on the same site, respectively, as in the standard Bose-Hubbard model. The third 
term represents the energy associated with spin configurations within lattice sites which penalizes non zero spin configurations in each individual lattice. This term will induce distinct Mott phases that differ from each other in their spin correlations [119]. The appearance of spin mediated tunneling transitions in the optical lattice depends clearly on the ratio between the different energy scales appearing on the Bose Hubbard Hamiltonian. We shall consider in what follows the polar superfluid phase characterised by $c_{2}>0$, and assume that spin-independent interactions $c_{0}$ are larger than the spin-dependent ones $c_{2}$ [119]. The superfluid-Mott transition depends then only on the ratio $t / c_{0}$. However, once the Mott regime is achieved, if $c_{2}>t$ one should expect different magnetic ordering in the insulator phase due to the spin interactions. On the contrary, if $t \gg c_{2}$ tunneling will manifest equal for all spin components and the gas will behave as a strongly correlated scalar gas. For small but finite tunneling $t / c_{0} \ll 1$ it is possible to perform perturbation theory. To derive an effective Hamiltonian to second order in $t / c_{0}$. We split, as usually, the full Hamiltonian as $H=H_{0}+H_{t}$ where $H_{0}=\left(c_{0} / 2\right) n(n-1)+\left(c_{2} / 2\right) \vec{F}^{2}-$ $2 n-\mu n$ describes the one site unperturbed Hamiltonian and $H_{t}$ describes tunneling between two adjacent sites, i.e we consider a two site problem. To derive the effective Hamiltonian one looks how the energy of the unperturbed ground states $|g, S\rangle$ is lowered due to tunneling: $\epsilon_{S}=-\sum_{\nu} \frac{\left|\left\langle\nu\left|H_{t}\right| g, S\right\rangle\right|^{2}}{E_{\nu}-E_{g}}$ where $\nu$ labels all the (virtual) intermediate states and $E_{\nu}, E_{g}$ denote the unperturbed energies of the two-site state $|\nu\rangle,|g, S\rangle$ (which are non-degenerate). The dependence of the energy shifts on the total spin $S$ introduces nearest-neighbor spin-spin interactions in the lattice. It is sufficient to evaluate these shifts for only one value of $m_{S}$ because tunneling cannot mix states with different $m_{S}$ and the overlaps $\left|\left\langle\nu\left|H_{t}\right| g, S\right\rangle\right|$ are rotationally invariant. The effective Hamiltonian can be written then as:

$$
H_{i j}=\sum_{0}^{F_{1}+F_{2}=S} \epsilon_{S} P_{S}
$$

where, now, the sum extends to all $F_{1}+F_{2}$ values. The effective Hamiltonian for $S=1$ in second order on $t$ can be expressed as a generalized quadratic Heisenberg Hamiltonian [119]:

$$
H_{i j}=-J_{0}-J_{1} \sum_{\langle i, j\rangle} \mathbf{F}_{\mathbf{i}} \mathbf{F}_{\mathbf{j}}-J_{2} \sum_{\langle i, j\rangle}\left(\mathbf{F}_{\mathbf{i}} \cdot \mathbf{F}_{\mathbf{j}}\right)^{2},
$$

where the explicit expressions for $J_{0}, J_{1}$, and $J_{2}$ can be found in Ref. [119].

This Hamiltonian differs from the familiar Heisenberg Hamiltonian for spin 1/2 particles due to the presence of the quadratic term on the spin interaction. Higher order terms on perturbation theory can give rise to terms with higher powers in $\left(\mathbf{F}_{\mathbf{i}} \mathbf{F}_{\mathbf{j}}\right)$ but with much smaller coefficients [120]. We will see, however, in the next subsection, that higher orders in the Heisenberg interactions appear in a perturbative treatment to second order if larger spins are used. We should ignore now higher order perturbative terms (i.e. $\propto t^{4}$ ) since their contribution are highly suppressed. We summarise the possible quantum phases in such a case. As reported by Imambekov et al. [119], for an optical lattice in 2D and 3D, the phase diagram can be summarized as follows. The term $J_{1}$ favors ferromagnetic order, while $J_{2}$ enhances the singlet spin configuration on each bond. To solve this competition, there is the 


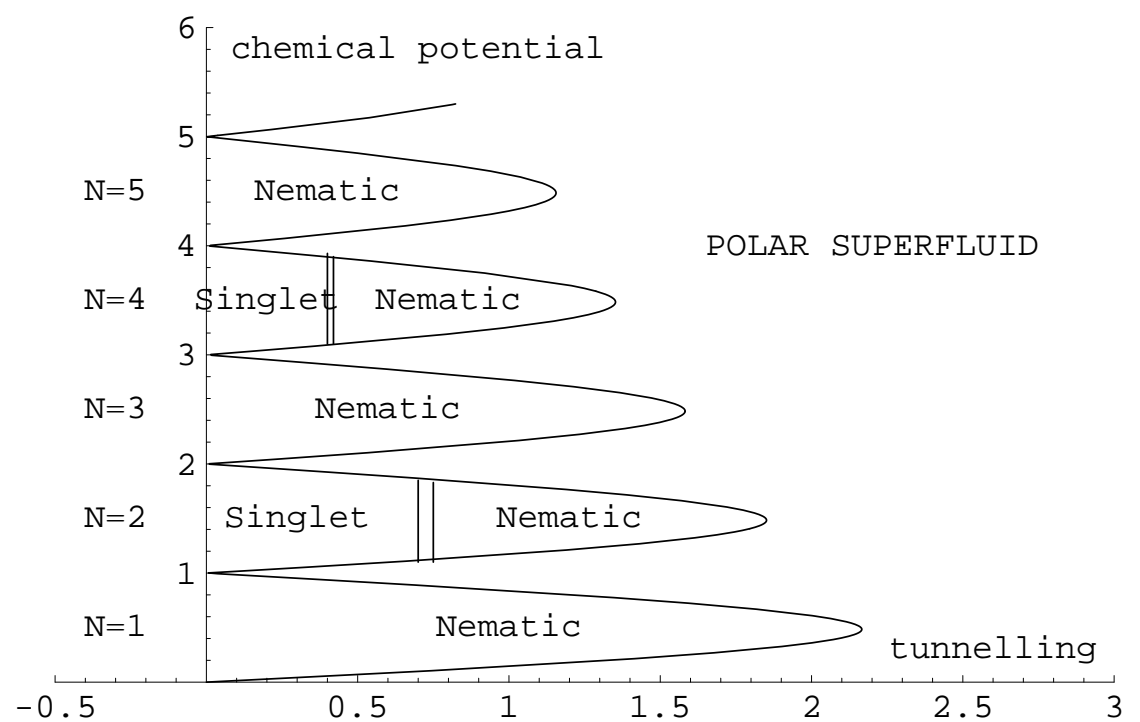

Figure 23. General phase diagram for $F=1$ bosons in 2D and 3D optical lattices (from [119]).

possibility of having nematic states that mix states with total spin $S=0$ and $S=2$ (and $m_{F}=0$ ) at each bond, but are product states:

$$
|N\rangle=\prod_{i}\left|F_{i}=1, m_{i}=0\right\rangle
$$

This is a nematic state, with zero expectation value of each spin but not rotationally invariant. For an odd filling factor, the Mott insulating phase is always nematic. For even filling factors, there is always a spin-singlet phase, in which pairs of atoms at a site form singlets. See Fig. 23.

Apart from nematic and singlet states, in 1D, an additional solution is the dimerized VBS state

$$
|D\rangle=\prod_{i=2 n}\left|F_{i}=1, F_{i+1}=1, F_{i}+F_{i+1}=0\right\rangle,
$$

in which the translational invariance is broken.

6.3.3 $F=2$ gases in optical lattices. The mean field states of spinor $F=2$ gases have been for the first time investigated in Refs. [510,514,515]. It is worth noticing that mean field states are also valid for insulating Mott states with one atom per lattice site, provided all atoms are described by the same single-particle wave function attached to a given site. Refs. $[510,515]$ go one step further, and apart from the mean field theory also consider the extreme case of quenched (immobile) 
$F=2$ bosons in an optical lattice. In other words, these references characterize possible on-site states for $N$ bosons with total spin $S$ in the absence of tunneling. After submission of the first version of this paper, Barnett, Turner and Demler have presented a beautiful and complete classification of the mean field phases for arbitrary $F$, based on the 19th century F. Klein's method of solving quintic polynomials by analysis of rotations of regular icosahedra [168]. We discuss their results in more details in the following. Also, very recently the effective spin Hamiltonians (in the first MI lobe), and quantum insulating phases of $F=2$ bosons have been studied by Zhou amd Semenoff [169], using variational principle applied to product (Guztwiller ansatz, cf. [29]), dimer and trimer states.

6.3.4 Bose-Hubbard model for $\boldsymbol{F}=2$ particles. The derivation of the BoseHubbard Hamiltonian for spin $F=2$ can be conveniently expressed [515] as:

$$
\begin{aligned}
H= & -t \sum_{<i j>, \sigma}\left(b_{\sigma i}^{\dagger} b_{\sigma j}+b_{\sigma j}^{\dagger} b_{\sigma i}\right)+\frac{c_{0}}{2} \sum_{i} n_{i}\left(n_{i}-1\right) \\
& +\frac{c_{1}}{2} \sum_{i}: \boldsymbol{F}_{i} \cdot \boldsymbol{F}_{i}:+\frac{2 c_{2}}{5} \sum_{i} \bar{S}_{+i} \bar{S}_{-i}
\end{aligned}
$$

where the : : denotes normal ordering of the operators.

The ratios between the various interactions, $c_{1} / c_{0}$ and $c_{2} / c_{0}$, are fixed by the scattering lengths whereas the ratio $t / c_{0}$ between tunneling and Hubbard interactions can be tuned by changing the lattice parameters [29]. The possible ground state phases for $F=2$ have been studied in [122] using second order perturbation theory with $t / c_{0} \ll 1$. At zeroth order, the Hamiltonian is a sum of independent single-site Hamiltonians:

$$
H_{0}=\frac{c_{0}}{2} n(n-1)+\frac{c_{1}}{2}: \boldsymbol{F} \cdot \boldsymbol{F}:+\frac{2 c_{2}}{5} \bar{S}_{+} \bar{S}_{-}
$$

Exact eigenstates of this Hamiltonian have been obtained in Ref. [515]. Since $S_{ \pm}$ commute with the total spin operator, the energy eigenstates can be labeled with four quantum numbers as $\left|N, N_{S}, F\right\rangle_{m_{F}}$. They have a $2 F+1$-fold degeneracy associated with the quantum number $m_{F}$ and their energies are given by:

$$
E=\frac{c_{0}}{2} N(N-1)+\frac{c_{1}}{2}(F(F+1)-6 N)+\frac{c_{2}}{5} N_{S}\left(2 N-2 N_{S}+3\right) .
$$

Assuming one atom per site, the effective Hamiltonian to second order in the perturbation parameter $t / c_{0}$ has the following form:

$$
H_{I}^{(i j)}=-4 t^{2}\left[\frac{P_{0}^{(i j)}}{g_{0}}+\frac{P_{2}^{(i j)}}{g_{2}}+\frac{P_{4}^{(i j)}}{g_{4}}\right] .
$$

Notice that $\epsilon_{S}=-4 t^{2} / g_{S}$ indicates that the control and engineering of the magnetic properties of the system could be easily achieved by means of e.g. optical Feshbach resonances. The Hamiltonian (Eq.(101)) can be easily generalized to the whole 
lattice, $H=\sum_{i} H_{0, i}+\sum_{<i j>} H_{I}^{(i j)}$. It can also be transformed into a polynomial of fourth order in the Heisenberg interaction $\mathbf{F}_{i} \cdot \mathbf{F}_{j}$ :

$$
\begin{aligned}
H= & \sum_{i} H_{0, i}+\sum_{<i j>} \frac{39 \epsilon_{0}-80 \epsilon_{2}}{51}\left(\mathbf{F}_{i} \cdot \mathbf{F}_{j}\right)+\frac{9 \epsilon_{0}-8 \epsilon_{2}}{102}\left(\mathbf{F}_{i} \cdot \mathbf{F}_{j}\right)^{2}+ \\
& \left(-\frac{7 \epsilon_{0}}{204}+\frac{10 \epsilon_{2}}{204}+\frac{\epsilon_{4}}{72}\right)\left(\mathbf{F}_{i} \cdot \mathbf{F}_{j}\right)^{3}+\frac{7 \epsilon_{0}+10 \epsilon_{4}}{1020}\left(\mathbf{F}_{i} \cdot \mathbf{F}_{j}\right)^{4} .
\end{aligned}
$$

Very recently, effective spin Hamiltonians (in the first MI lobe) and quantum insulating phases of $F=2$ have been studied in [169].

To study the ground state phase diagram of a one-dimensional $F=2$ spinor gas assuming that there is the freedom to modify the value of (the zero magnetic field) scattering lengths [122], one can use Matrix Product States (MPS) methods. MPS represent the ground state of a translationally invariant short range Hamiltonian exactly [311], or nearly exactly (see subsection 3.10.2). A straightforward way to do that [530] is to add to the bond Hamiltonian $(101) c(>0)$ times the identity operator $I^{(i j)}=\sum_{S} P_{S}^{(i j)}$ on the bond, so that the new Hamiltonian $H^{\prime}=H_{I}^{(i j)}+c I$ becomes positive definite,

$$
H_{I}^{\prime(i j)}=\sum_{S=0}^{4} \lambda_{S} P_{S}^{(i j)},
$$

i.e., all $\lambda_{S}$ are non-negative. In particular, $\lambda_{1}=\lambda_{3}=c$ and $\lambda_{0}=c-4 t^{2} / g_{0}$, $\lambda_{2}=c-4 t^{2} / g_{2}$ and $\lambda_{4}=c-4 t^{2} / g_{4}$. Since by definition, $\lambda_{1}=\lambda_{3}$ are the largest parameters, the ground state will obviously belong to the symmetric subspace.

Before we proceed, it is worth to discuss the mean field phase diagram obtained under the assumption that the ground state is a product state, $|\Psi\rangle=|e, e, \ldots\rangle$ (see $[168,514])$. There are 3 possible mean field (i.e., product) ground states, with $|e\rangle$ given (up to $S O(3)$ rotations) by:

- Ferromagnetic state, $|e\rangle=(1,0,0,0,0)$; possesses only the $U(1)$ symmetry of rotations around the $z$-axis, and has maximal projection of the spin onto $z$ axis.

- nematic state, has the $\eta$-degeneracy, $|e\rangle=(\sin (\eta) / \sqrt{2}, 0, \cos (\eta), 0, \sin (\eta) / \sqrt{2})$. This state is a MI version of the polar state in BEC; it has mean value of all components of the spin equal zero, but non vanishing singlet projection $\langle$ singlet $\mid \mathrm{e}, \mathrm{e}\rangle \neq 0$;

- Tetrahedratic (cyclic) state, $|e\rangle=(1 / \sqrt{3}, 0,0, \sqrt{2 / 3}, 0)$; this is a MI version of the cyclic state. The state may be uni- or biaxial, depending on whether the nematic tensor does, or does not have a pair of degenerated eigenvalues; it has vanishing of both, of mean values of all of the spin components, and of the singlet projection.

The mean field phase diagram corresponds to:

- a ferromagnetic state for $\lambda_{4}=0, \lambda_{2}, \lambda_{0}>0$, and for $\lambda_{0}=0$, provided $\lambda_{2} \geq$ $17 \lambda_{4} / 10$;

- a nematic state for $\lambda_{0}=0$, provided $3 \lambda_{4} / 10 \lambda_{2} \leq 17 \lambda_{4} / 10$;

- a cyclic state for $\lambda_{0}=0$, provided $\lambda_{2} \leq 3 \lambda_{4} / 10$, and for $\lambda_{2}=0$.

In $1 \mathrm{D}$, since the Hamiltonian is a sum of nearest neighbor bond Hamiltonians, we have $\sum_{k, k^{\prime}}\left\langle e_{k}, e_{k} \ldots\left|\hat{H}_{I}\right| e_{k^{\prime}}, e_{k^{\prime}} \ldots\right\rangle \propto\left\langle e_{k} \mid e_{k^{\prime}}\right\rangle^{N-2}$ and thus in the limit of an 
infinite chain the ground states are equally well described by product states (that will typically break the rotational symmetry). This means in this case we expect mean field product states to provide a very good approximation of the ground states with translational symmetry. Below we present a schematic classification of the possible ground states for the specific $\lambda_{i}$ values [122].

(A) For $\lambda_{4}=\lambda_{2}=\lambda_{0}=0$, all symmetric states are ground states, i.e., in particular all product states $|e, e \ldots\rangle$ with arbitrary $|e\rangle$.

(B) For $\lambda_{4}=\lambda_{2}=0, \lambda_{0}>0$, the ground states $|e, e \ldots\rangle$ remind the cyclic states states of Ref. [118] (i.e., they correspond to translationally, but nor rotationally invariant product states), which now mix $S=2$ and $S=4$ contributions on each bond, and they have to fulfill the condition $\langle$ singlet $\mid e, e\rangle=0$. Denoting by $|e\rangle=\left(e_{2}, e_{1}, e_{0}, e_{-1}, e_{-2}\right)$, this implies $e_{0}^{2}-2 e_{1} e_{-1}+2 e_{2} e_{-2}=0$. These states form a much greater class than the cyclic ones, since they may have non-vanishing (and even maximal) components of the spin. Interestingly, the transition between the cyclic phase for $\lambda_{2}=0$, and the ferromagnetic phase for $\lambda_{4}=0$, occurs via such states, i.e., at the transition point the degeneracy of the ground states manifold explodes.

$(C)$ For $\lambda_{4}=0$ and $\lambda_{2}, \lambda_{0}>0$, the ground states are ferromagnetic states $|2\rangle_{\mathbf{n}}|2\rangle_{\mathbf{n}} \cdots|2\rangle_{\mathbf{n}}$, corresponding to a maximal projection of the local spin onto a given direction $\mathbf{n}=(\sin (\theta) \cos (\phi), \sin (\theta) \sin (\phi), \cos (\theta))$. Such vectors for $F=2$ may be parametrized (in the basis of $\hat{\mathbf{F}}_{\mathbf{n}}$ with descending $m_{F}$ ) as $\propto\left(z^{-2}, 2 z^{-1}, \sqrt{6}, 2 z, z^{2}\right)$ with $z=|z| e^{i \phi},|z| \in(-\infty, \infty)$. It should be stressed that ferromagnetic states are exact ground state in the entire part of the phase diagram whenever $\lambda_{4}=0$.

(D) For $\lambda_{0}=0$ and $\lambda_{4}, \lambda_{2}>0$, the ground states apparently favor antiferromagnetic order. This, however, can be misleading if $\lambda_{4} \ll \lambda_{2}$. In that case, as the mean field diagram suggests, the ferromagnetic order might prevail. We have applied in 1D a more general variational approach, going beyond mean field. We have looked for ground states by applying the variational principle to mean field (product) states $|e, e \ldots\rangle$, Néel-type states $|e, f, e, f \ldots\rangle$, and valence bond solid states with singlet states for distinct pairs (dimers) of neighboring atoms and translational dimer symmetry. For the mean field case as discussed earlier the energy is either minimized by the ferromagnetic state $|e\rangle=|2\rangle_{\mathbf{n}}$ (for $\lambda_{2} \geq 17 \lambda_{4} / 10$ ), by a nematic state $|e\rangle=|0\rangle_{\mathbf{n}}$ (for $3 \lambda_{4} / 10 \lambda_{2} \leq 17 \lambda_{4} / 10$; in this case the state is a combination of total spin 0,2 and 4$)$, or, for $\lambda_{2} \leq 3 \lambda 4 / 10$, by a cyclic state, $|e\rangle=\left(e_{2}, e_{1}, e_{0}, e_{-1}, e_{-2}\right)$ with $e_{0}=1 / \sqrt{2}, e_{2}=-e_{-2}=1 / 2, e_{1}=e_{-1}=0$. Imposing Néel order with $\langle e \mid f\rangle \neq 1$ always results in a larger energy, as $\lambda_{1,3}>\lambda_{2,4}$, and the overlap with the singlet can be maximized already by restricting to product states. On the other hand, for the dimer state the energy per bond is given by $\frac{1}{2} \operatorname{Tr}\left(H_{I} \frac{1}{25} 1 \otimes 1\right)$. One can apply MPS codes [122] to search numerically for the exact ground states in 1D using the method of [311] to confirm the existence and phase borders of the ferromagnetic ground state, nematic and dimer regions. This particular phase diagram in the $\lambda_{2}, \lambda_{4}$ phase space is displayed in Fig. 24 .

$(E)$ For $\lambda_{2}=0$ and $\lambda_{4}, \lambda_{0}>0$, as in the $(D)$ case, mean field cyclic states are favorable over Néel states. One can compare them variationally to the analogues of the dimer states in the present case, i.e., configurations which have a state with total spin $S=2$ on distinct bonds. We call these state para-dimers. Now, contrary to the dimerized states in $(D)$, where the states on the bond are unique, here states with 


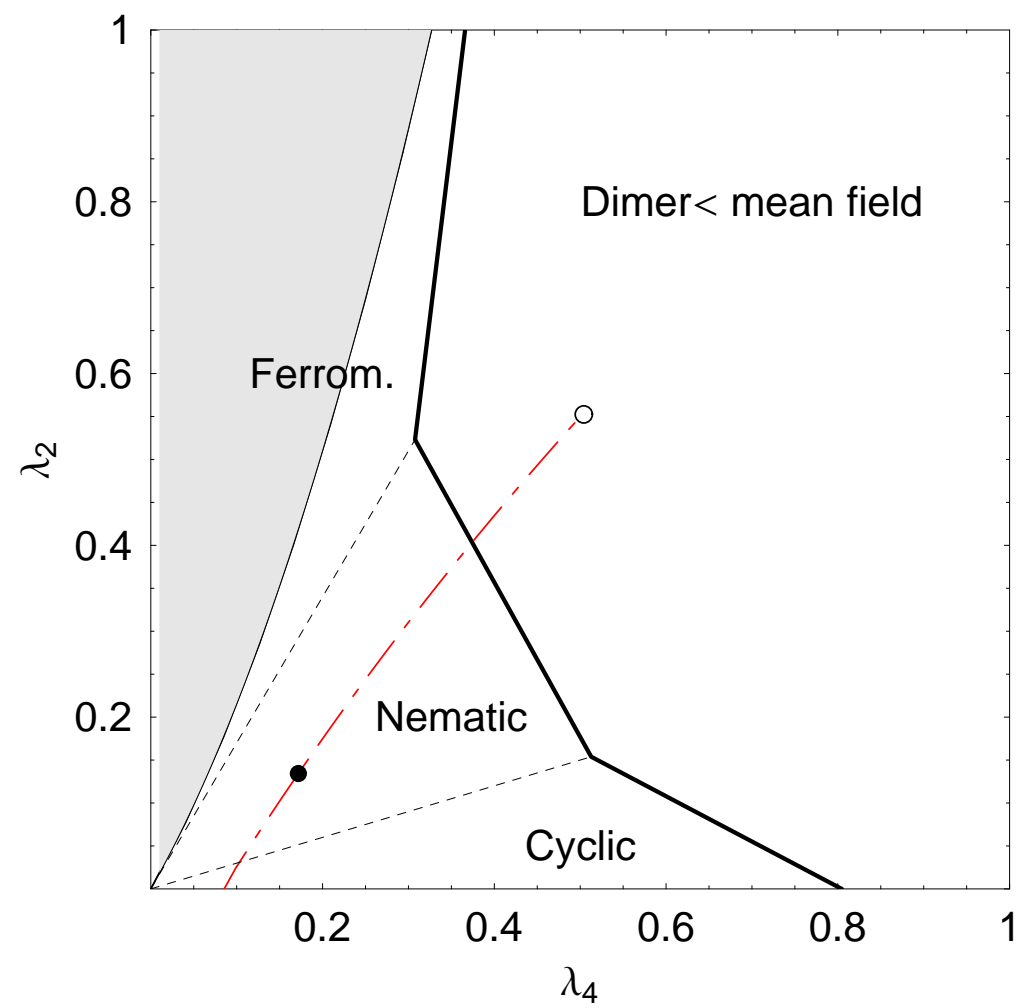

Figure 24. (Color online.) Sketch of the phase diagram, obtained by applying the variational principle in the $\lambda_{2}, \lambda_{4}$ phase space (for $\lambda_{0}=0$ ) to mean field, Néel, and dimer states with one atom per site. The scale is set by letting $\lambda_{1}=\lambda_{3}=1$. Néel-type states are never favorable over nematic states. The ferromagnetic region (gray) was obtained numerically by imaginary time evolution of MPS (and comparing the results from runs with $D=1$ and $D=5$ ) in a chain of 50 sites with open boundary conditions [300]. (Note that here $D$ is the dimension of the ancillary system that is used in the MPS method.) Of course, on the line $\left(\lambda_{4}=0, \lambda_{2}\right)$ ferromagnetic states give always ground states. Dashed lines indicate the regions where the type of mean field state with lowest energy changes qualitatively. The red (dashed-dotted) line gives the values of $\left(\lambda_{2}, \lambda_{4}\right)$ which can be obtained by changing the spin-independent scattering length $c_{0}=\left(3 g_{4}+4 g_{2}\right) / 7$ through optical Feshbach resonances. [175,177]. Black and white circles indicate a change of $c_{0}$ of $10 \%$ and $100 \%$, respectively.

different $m_{S=2}$ can form superpositions. It is easy to see, however, that the best superposition should have maximal possible entanglement, in order to minimize interaction on the bonds that are not covered by the para-dimers [122].

6.3.5 Spinor Fermi gases in optical lattices. We move now to fermionic spinor gases. For $F=1 / 2$, the quantum simulator of the fermionic Hubbard model has been proposed in [90] and recently studied with 3-component fermions in [531]. Liu et al. [41] proposed to use fermions with high $F$ to realize spin-dependent Hub- 
bard models, in which hopping parameters are spin-dependent. Such models lead to exotic kinds of superfluidity, such as to a phase in which SF and normal component coexist at zero temperature. W. Hofstetter and collaborators have written a series of papers, reviewed in [532], on fermionic atoms with $S U(N)$ symmetry in optical lattices. Such systems also have exotic superfluid and flavor-ordered ground states, and exhibit a very rich behavior in the presence of disorder. It is, of course, inevitable to ask which atoms can be used to realize high $F$ fermonic spinor gases in optical lattices. The most commonly used alkali ${ }^{6} \mathrm{Li}$ has hyperfine manifolds with $F=1 / 2$ and $F=3 / 2$. The latter is, obviously, a subject of two body losses, but as in the case of the $F=2$ manifold of Rubidium, one can expect reasonably long life time in the lattice (especially in MI states with $N=1$ ). Another commonly used fermion is a heavy alkali ${ }^{40} \mathrm{~K}$, which has manifolds $F=7 / 2$ and $F=9 / 2$. These fermions are particularly useful for spin-dependent Hubbard models [41]. There are several atoms whose lowest hyperfine manifold is $F=3 / 2$, i.e., in those ground states two body losses can be avoided: ${ }^{9} \mathrm{Be},{ }^{132} \mathrm{Cs}$, or ${ }^{135} \mathrm{Ba}$, but so far only the bosonic Cesium BEC has been achieved [533]. On the other hand, recently a BEC of ${ }^{174} \mathrm{Yb}$ atoms [534], as well as degenerate gas of ${ }^{173} \mathrm{Yb}$ fermions with $F=5 / 2$, has been realized. Finally, fermionic Chromium has 4 hyperfine manifolds with $F=9 / 2,7 / 2,5 / 2,3 / 2$, in the ascending order of energies, and after achieving BEC of the bosonic Chromium [145], the prospect for achieving ultracold degenerate spinor Fermi gases are very good.

Recently, there has been a lot of progress in understanding the special properties of $F=3 / 2$ and $F=5 / 2$ lattice Fermi gases [122,535, 536]. C. Wu et al. [537] realized that the spin- $3 / 2$ fermion models with contact interactions have a generic $S O(5)$ symmetry without any fine-tuning of parameters, and employed this fact to form a quantum Monte Carlo algorithm free of the sign-problem and to study novel competing orders in 1-dimensional optical traps and lattices [538]. In particular, the quartetting phase, a four-fermion counterpart of Cooper pairing, exists in a large portion of the phase diagram. The bosonisation approach was applied to 1D systems with $F=3 / 2,5 / 2, \ldots$ by Lecheminant et al. [207], and exact Bethe ansatz in [208]. An overview of hidden symmetries and competing orders in spin $3 / 2$ gases can be found in the excellent paper of $\mathrm{C}$. Wu [539].

\section{Ultracold atomic gases in "artificial" magnetic fields}

\subsection{Introduction - Rapidly rotating ultracold gases}

Quantum systems in magnetic fields exhibit particularly interesting behavior, with fractional quantum Hall effect (FQHE) being a paradigm [540]. It is very well known that rapidly rotating trapped ultracold gases provide a possibility to realize "artificial" (standard, or better to say, Abelian) magnetic fields.

In order to achieve such magnetic fields, one typically considers a quasi single component ("spinless") 2D gas of $N$ atoms in the $X Y$ plane rotating around the $Z$ axis with frequency $\Omega$, and confined in a harmonic trap of frequency $\omega_{\perp}$. If the rotation is moderate $\Omega<\omega_{\perp}$, in macroscopic atomic clouds, the Abrikosov vortex lattice is formed $[541,542]$. As $\Omega$ approaches $\omega_{\perp}$, the vortex lattice melts, and the system evolves through a sequence of states that have been identified in 
literature $[125,127,543]$ as highly correlated quantum liquids.

The various regimes of rapidly rotating gases can be described in the terminology of fractional quantum Hall effect (FQHE) theory [540]. The crucial role is played by the direct analog of the Landau level filling factor in the FQHE, which can be related to the number of vortices $N_{v}$ by $\nu=N / N_{v}$ as defined in the BEC mean field description, which is valid for large systems and moderate rotations.

In the first literature $[125,127]$, the authors considered only the lowest Landau level (LLL) for strong enough rotation. Later, attention was paid to edge excitations and topological order [544]. Recently, correlated liquids at $\nu=k / 2$ for $k=1,2,3, \ldots$ for $\nu \leq \nu_{c} \simeq 6-10$ have been discussed [126,545-547]. These states resemble, to a great extent, the states from the Rezayi-Read (RR) hierarchy [548]: $k=1$ is the Laughlin state, $k=2$ is the Moore-Read paired state [544,549], etc. Rezayi et al. [550] have recently shown that the presence of a small amount of dipole-dipole interactions unambiguously makes the $\mathrm{RR}$ state with $k=3$ the ground state. This state is particularly interesting, since its excitations are both fractional, and nonAbelian. The validity of the LLL approximation for rotating gases is also discussed in [551]. Recently, a composite fermion (boson + one flux quantum) theory of rapidly rotating bosons has been formulated [552].

Most of the literature on ultracold rapidly rotating gases aims at considering relatively large systems and even the thermodynamic limit. Thus, in numerical simulations, either periodic (torus) or spherical boundary conditions are used. Stability of the cloud in an harmonic trap requires $\Omega<\omega_{\perp}$, since otherwise centrifugal forces drag the atoms away from the trap. Observation of the Laughlin states requires, on the other hand, to remain in the LLL. This in turn can be assured only if $\Omega-\omega_{\perp}=O(1 / N)$, i.e., it requires a very precise control of the delicate balance between $\Omega$ and $\omega_{\perp}$. Unfortunately, in the case of contact (short range Van der Waals) interactions pseudo-hole excitations of the Laughlin state have vanishing interaction energy, similarly as the Laughlin state itself; they can differ only by angular momentum contribution $\propto \Omega-\omega_{\perp}=O(1 / N)$, i.e. they vanish at large $N$. Despite the progress in experimental studies of vortex lattices [553,554], and first steps toward reaching the LLL physics [555-557], experiments have not yet reached this regime.

The problems related to the short range nature of the Van der Waals forces can be overcome by using optical traps more stiff than harmonic, which is technically difficult, but in principle possible. Yet another promising idea is to use dipolar gases, i.e. gases that interact via magnetic or electric dipole moments (for a review see [144]). Rotating dipolar bosonic gases are expected to exhibit exotic behaviour in the weakly interacting regime [149], whereas fermionic dipolar gases have a finite gap for the $\nu=1 / 3$ Laughlin state [150]. The first observation of BEC of a dipolar gas of Chromium atoms with large magnetic dipole has been recently reported [179], and several groups are trying to achieve an ultracold gas of heteronuclear molecules with large electric dipole moments [558]. The recent proposal of using polar molecules excited to the first rotational state is also very promising [115].

Completely different approach to create "artificial" magnetic fields employ the effect of electromagnetically induced transparency (EIT) and "slow light"; it has been proposed by Juzeliunas and Öhberg [559-562]. This method is very flexible, and can be used to reach the integer quantum Hall effect [563], and even regimes of FQHE in planar geometries [562], or generate non-Abelian gauge fields [136]. 
Nevertheless, atomic gases in optical lattices, or arrays of optical traps provide perhaps the best opportunity for FQHE. There are two ways of reaching the regime of Laughlin liquids. A very promising one, consists in using atoms in an array of rotating optical microtraps, either in an optical lattice [564], or created by an array of rotating traps generated by an array of microlenses [565], or an array of laser beams [566].

In such arrangements, it will be natural to study independent mesoscopic or even microscopic systems of few atoms at each lattice site, i.e., avoid the problems of large $N$. The lattice will play, however, an important role. First of all, one can prepare equal number of atoms at each site using the Mott transition, and then turn the intersite hopping off. Second, the measurement and detection procedures could automatically be applied to many copies of the same meso-, or micro-system. Such experiments demand careful theoretical studies of few atom systems using possibly exact methods, such as exact diagonalisations of the Hamiltonian with open boundary conditions in the presence of the harmonic trap, or even a deformed trap. Such studies have been initiated recently. Popp et al. [128] analyzed in detail the possibilities of an adiabatic path to fractional quantum Hall states of few bosonic atoms. The papers of Barberan et al. [129] discuss the appearance of ordered structures (vortices, vortex arrays, Wigner crystals) in systems of few bosonic atoms by looking at density and pair-correlation functions. In Fig. 25, an example of such structures pair correlation function in the Laughlin states is shown. Similar studies (going even beyond LLL approximation) for bosonic and fermionic atoms have been reported in Ref. [567].

Another way to create highly-correlated liquids could be to mimic effects of magnetic fields not by rotation, but by appropriately designed control of tunnelling in optical lattices. This is discussed in the next subsection.

\subsection{Lattice gases in "artificial" Abelian magnetic fields}

In order to create an effect of constant magnetic field in a $2 \mathrm{D}$ square lattice one needs to be able to control the phases of the hopping matrix elements. Single atom stationary Schrödinger equation, analyzed in the famous paper by Hofstadter [568], reads:

$$
\begin{aligned}
& -t e^{-\mathrm{i} \frac{\mathrm{e} a}{\hbar c} A_{x}} \psi(x+a, y)-t e^{\mathrm{i} \frac{\mathrm{e} a}{\hbar c} A_{x}} \psi(x-a, y) \\
& -t e^{-\mathrm{i} \frac{\mathrm{e} a}{\hbar c} A_{y}} \psi(x, y+a)-t e^{\mathrm{i} \frac{\mathrm{e} a}{\hbar c} A_{y}} \psi(x, y-a)=E \psi(x, y),
\end{aligned}
$$

where $\psi(x, y)$ is the wave function, $a$ is the lattice constant, and $\vec{A}$ is the vector potential. The choice of $\vec{A}$ determines the magnetic field $\vec{B}$, which in turn determines the behavior of the system. For uniform field in the $z \operatorname{direction,} \vec{B}=B \overrightarrow{\mathrm{e}}_{z}$, one may choose the potential $\vec{A}=(0, B x, 0)$, and tunnelings in the $y$-direction acquire phases. This makes the problem effectively one-dimensional and Eq. (104) can be transformed into the Harper's equation [569]:

$$
g(m+1)-g(m-1)+2 \cos (2 \pi m \alpha-\nu) g(m)=\varepsilon g(m)
$$

by using the ansatz $\psi(m a, n a)=\mathrm{e}^{\mathrm{i} \nu n} g(m)$, where $x=m a, y=n a$, and $\varepsilon=-E / t$. 

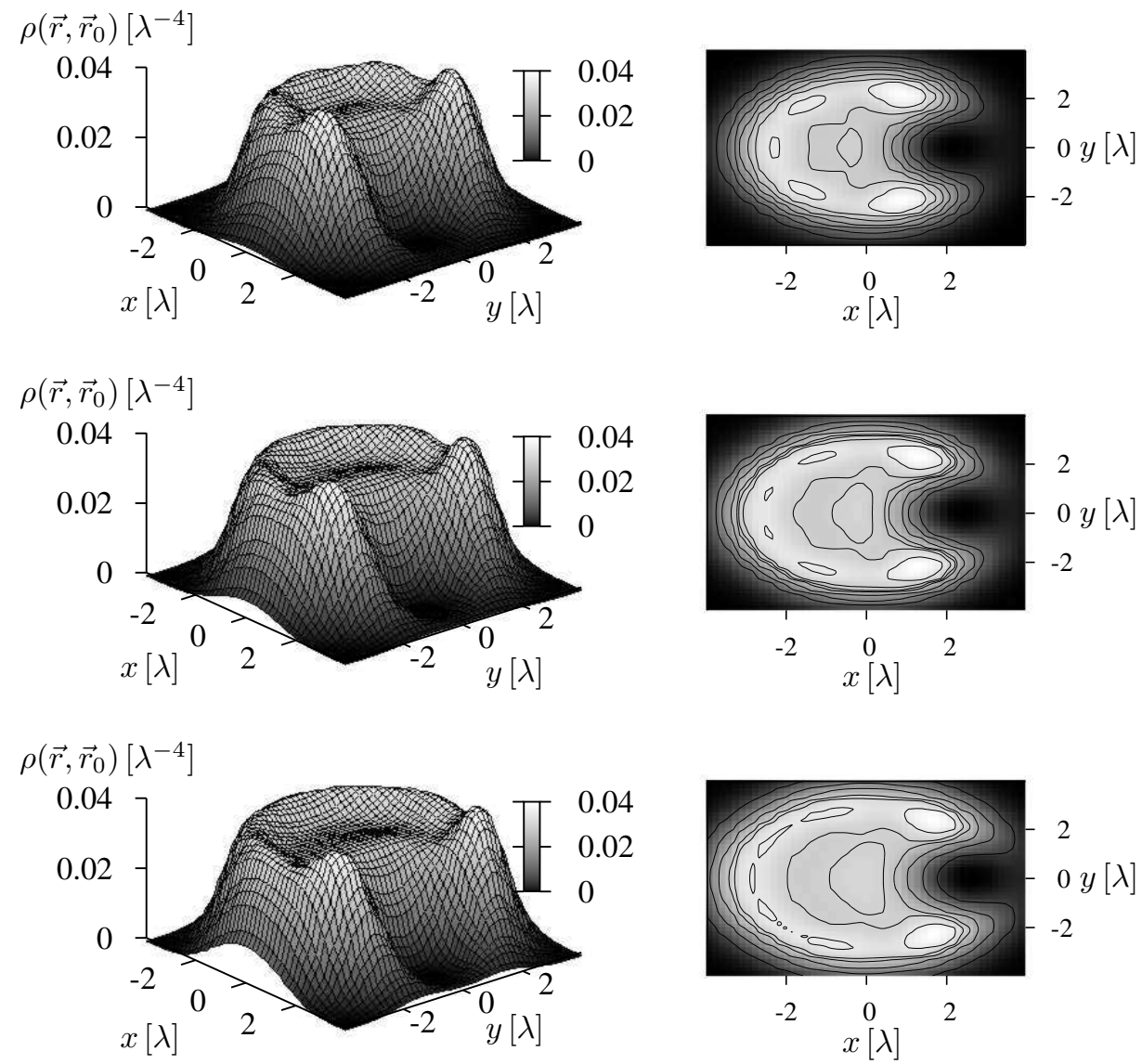

Figure 25. 3D (left) and contour (right) plot of the pair correlation function $\rho\left(r, r_{0}\right)$ for the Laughlin states of $N=6,7$ and 8 atoms of mass $m$ in a lattice site; $r_{0}=\sqrt{N}$ in harmonic oscillator units and $\lambda=\sqrt{\hbar / 2 m \omega \perp}$ (from [129]).

The eigenvalue problem for rational values of the magnetic flux $\alpha=\frac{\mathrm{e} a^{2} B}{h c}$ per elementary plaquette becomes periodic. This results in a band spectrum whose bands form the famous Hofstadter butterfly (see Fig. 26). Note that the regime of this spectrum requires finite values of $\alpha$, i.e., magnetic fields $B \sim 1 / a^{2}$, which in the continuum limit $a \rightarrow 0$ become ultra-intense.

Jaksch and Zoller [130], were the first who have recently proposed methods to realize such "artificial" magnetic field effects in lattice gases. Their method employs atoms with two internal states trapped in two lattices, laser assisted tunneling, lattice tilting (achieved by acceleration, or application of external static electric fields), and other experimentally accessible techniques. We discuss the generalization of this proposal to the case of non-Abelian fields in the next subsection. 


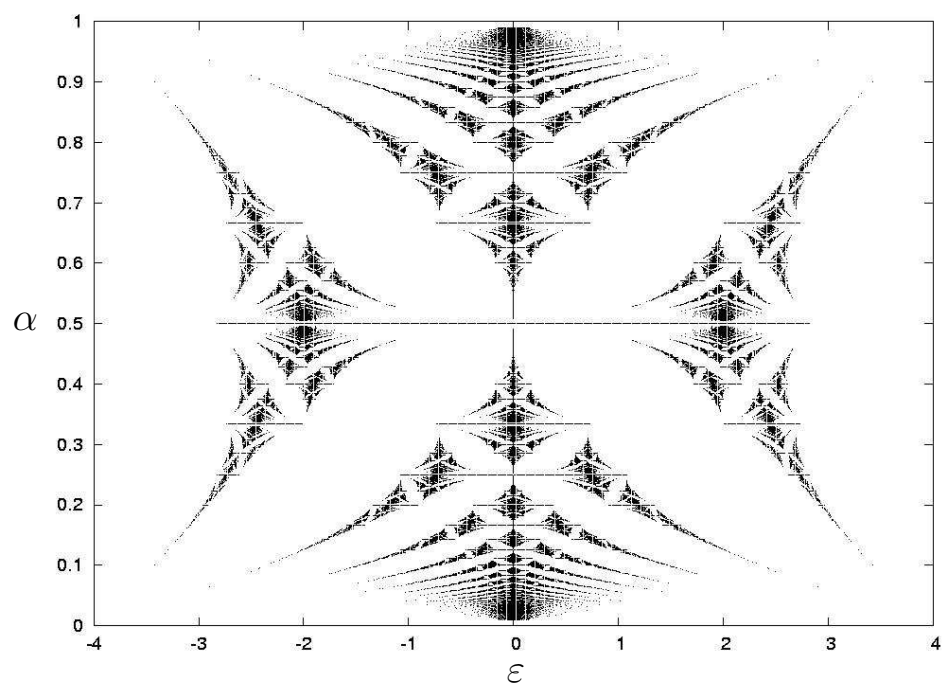

Figure 26. The famous Hofstadter butterfly: energy bands plotted for rational values of the magnetic flux $\alpha$.

The physical features in "artificial" magnetic fields are, as expected extremely rich. For instance, one may load the lattice with a cold BEC, or add disorder, and observe how the single atom properties of the spectrum changes in weakly interacting or weakly disordered systems. The modifications of the butterfly due to interactions and disorder, or both respectively, can be measured, as proposed in [130].

Several other groups made alternative proposals for "artificial electromagnetism for neutral atoms". E.J. Mueller [131] generalized the method of [130] to atoms with 3 internal states in three lattices. The advantage of this approach is that it does not use lattice tilting, but on expense of more complicated laser configuration. Since the fields are "artificial" they do not even need to fulfill Maxwell equations, and can thus lead to physical realisations of otherwise unphysical effects. Mueller proposes for instance time dependent realisations of hopping matrix element on a 1D ring that leads to "Escher staircase": a single particle in such ring undergoes acceleration limited only by the Umklapp process: when the de Broglie wavelength of the particle becomes equal to the lattice constant, the matter wave is Bragg reflected off the lattice and reverses its direction.

Sorensen et al. [132] have proposed yet another method that employs also time dependent hopping matrix elements along with a large oscillating quadrupolar potential. These authors performed exact diagonalisations of the resulting Bose-Hubbard model in the limit of hard core bosons for $N$ up to 5 . In the limit of small $\alpha$ and low densities, the continuum limit may be applied and the model reduces then to a system of bosons in a magnetic field with contact interactions. For filling factor $1 / 2$ (total angular momentum $L=2 N$ ), the Laughlin wave function is then the exact ground state [448]. The numerical calculations show that the exact ground state remains very similar to the Laughlin state for $\alpha<0.3$. Preparation of such Laughlin state in the lattice may be achieved by creating first a Mott state with 
one atom per site in a superlattice with the larger period. In this way low density is achieved, while the atoms are quenched and not affected by the "magnetic" field. By turning off the superlattice, the Mott state melts into a Laughlin liquid.

Very recently, Palmer and Jaksch studied high field FQHE in optical lattices. They considered the value of $\alpha \simeq \alpha_{c}=l / n$, where $l, n$ are small integers, and derived the corresponding effective Hamiltonian in the continuum limit, which for $\alpha_{c}=1 / 2$ reduces to a model similar to a bilayer FQHE system [570]. The corresponding ground state in homogeneous systems (in the absence of trapping potential) is, the, so called, 221 state, constructed as the Laughlin state for $\nu=1 / 2$ for particles from the same layer, and for $\nu=1$ for particles from different layers. Denoting the coordinates by $z_{i}, s_{j}$, the wave function for $N=2 M$ atoms is

$$
\Psi\left(z_{1}, \ldots, z_{M}, s_{1}, \ldots, s_{M}\right) \propto \prod_{i \neq j}\left(z_{i}-z_{j}\right)^{2} \prod_{i \neq j}\left(s_{i}-s_{j}\right)^{2} \prod_{i, j}\left(z_{i}-s_{j}\right) .
$$

The angular momentum of this state is $L=2 M(M-1)+M^{2}$, and filling factor $\nu=2 / 3$. Interestingly, the Hall current in such states in the presence of the trap might exhibit unexpected sign changes.

Bukov and Demler [571] have recently studied "vortices" in a dice lattice, induced by "magnetic field" and have shown fascinating possibilities of creation of vortexPeierls state (a bosonic analog of valence bond solids). Rotating optical lattices has been considered by several authors: Polini et al. [572] proposed a realization of a fully frustrated XY model with cold atoms in optical lattices, while Bhat et al. [573] studied ground states of various symmetries for rotating $4 \times 4$ lattices. The somewhat related problem of vortex configuration in systems of rotating ultracold atoms in optical lattices has been studied by Wu et al. [574], who finds that near the superfluid-Mott insulator transition, the vortex core has a tendency to approach the Mott insulator state.

\subsection{Lattice gases in "artificial" non-Abelian magnetic fields}

In a recent Letter [135], it has been shown that by using atoms with more internal states, the application of state dependent, laser assisted tunneling (cf. [41]), and coherent transfer between internal states, one can generalize results of Jaksch and Zoller and create "artificial external magnetic fields" corresponding to non-Abelian $U(n), S U(n)$, or even $G L(n)$ gauge fields. In this case, the tunneling amplitudes are replaced by (unitary) matrices whose product around a plaquette is non-trivial and its mean trace (Wilson loop) is not equal to $n[134,575]$.

We consider an atomic gas in a 3D optical lattice and assume that tunneling is completely suppressed in the $z$-direction, so that, effectively, we deal with an array of 2D lattice gases and we are able to restrict ourselves to one copy. The atoms occupy two internal hyperfine states $|g\rangle,|e\rangle$, and the optical potential traps them in the state $|g\rangle$ and $|e\rangle$ in every second column, i.e., for the $y$ coordinate equal to $\ldots, n-1, n+1, \ldots(\ldots, n, n+2, \ldots)$. The resulting $2 \mathrm{D}$-lattice has thus the spacing $\lambda / 2(\lambda / 4)$ in the $x-(y-)$ direction. The tunneling rates in the $x$ direction are due to kinetic energy; they are spatially homogeneous and assumed to be equal for both hyperfine states. The lattice is tilted in the $y$-direction, which introduces an energy shift $\Delta$ between neighboring columns. Tilting can be achieved by accelerating the 
lattice, or by placing it in a static electric field. By doing this, standard tunneling rates due to kinetic energy are suppressed in the $y$ direction. Instead, tunneling is laser assisted, and driven by two pairs of lasers resonant for Raman transitions between $|g\rangle$ and $|e\rangle$, i.e., $n \leftrightarrow n \pm 1$. This can be achieved because the offset energy for both transitions is different and equals $\pm \Delta$. Detunings of the lasers are chosen in such a way that the effect of tilting is cancelled in the rotating frame of reference. The lasers generate running waves in the $\pm x$-direction, so that the corresponding tunneling rates acquire local phases $\exp ( \pm i q x)$.

In order to realize "artificial" non-Abelian fields in a similar scheme, one may use atoms with degenerate Zeeman sublevels in the hyperfine ground state manifolds, $\left|g_{i}\right\rangle$, and $\left|e_{i}\right\rangle$ with $i=1, \ldots, n$, whose degeneracy is lifted in external magnetic fields. These states may be thought of as "colors" of the gauge fields. Promising fermionic candidates with these properties are heavy Alkali atoms, for instance, ${ }^{40} \mathrm{~K}$ atoms in states $F=9 / 2, m_{F}=9 / 2,7 / 2, \ldots$, and $F=7 / 2, m_{F}=-7 / 2,-5 / 2, \ldots$; in particular, they allow for realizing "spin" dependent lattice potentials and hopping $[41]$.

Having identified the "colors", one modifies the scheme of Ref. [130]: laser assisted tunneling rates along the $y$-axis should depend on the internal state, although not necessarily in the sense of Ref. [41]. For a given link $\left|g_{i}\right\rangle$ to $\left|e_{i}\right\rangle$, tunneling should be described by a non-trivial unitary matrix $U_{y}(x)$ being a member of the "color" group $\left(U(n), S U(n), G L(n)\right.$ etc.). For unitary groups, the tunneling matrix $U_{y}(x)$ can be represented as $\exp \left(i \tilde{\alpha} A_{y}(x)\right)$. Here, $\tilde{\alpha}$ is real, and $A_{y}(x)$ is a Hermitian matrix from the gauge algebra, e.g., $U(n)$ or $S U(n)$. Since transitions from $\left|g_{i}\right\rangle$ to $\left|e_{i}\right\rangle$ correspond to different frequencies for each $i$, they are driven by different running wave lasers, and may attain different phase factors $\exp \left( \pm i q_{i} x\right)$.

In order to create gauge potentials that cannot simply be reduced to two independent Abelian components, tunneling in the $x$-direction should be described by a tunneling matrix $U_{x}$, which fulfills $\mathrm{t}\left[U_{x}, U_{y}(x)\right] \neq 0$, so that a genuine non-Abelian character of the fields is assured. We stress that all elements of this scheme, as shown in Fig. 27, are experimentally accessible.

For the specific gauge fields considered in Ref. [135] one obtains the following $U(2)$ generalization of the Harper's equation:

$$
\sigma_{x} g(m+1)-\sigma_{x} g(m-1)+2 \cos (2 \pi m \alpha-\nu) g(m)=\varepsilon g(m),
$$

where $g(m)$ is the two component wave function obtained by using the ansatz $\psi(m a, n a)=\mathrm{e}^{\mathrm{i} \nu n} g(m)$, with $x=m a, y=n a$, and $\varepsilon=-E / t ; \sigma_{x}$ is the Pauli matrix, and the "magnetic flux" matrix $\alpha=\operatorname{diag}\left[\alpha_{1}, \alpha_{2}\right]$.

Given each $\alpha_{i}=p_{i} / q_{i}$ rational, the problem is $Q$-periodic (where $Q$ equals the smallest common multiple of $q_{1}$ and $q_{2}$ ). The spectrum shows a band structure, and is bounded by two hyperplanes (Fig. 28). It exhibits a very complex formation of holes of finite measure and various sizes, which we name the Hofstadter "moth". Although a rigorous proof can not be provided, the "moth" reminds a fractal structure. Obviously, this fractal structure will be very sensitive to any sort of perturbation (finite size of the system, external trapping potential etc.) on very small scales. But, since the holes are true 3D objects with finite volume, the spectrum will be more robust on a larger scale to perturbations than in case of the Hofstadter "butterfly". Very recently, the same gauge fields were used to investigate metal-insulator transition for cold atoms [576] and integer quantum Hall 


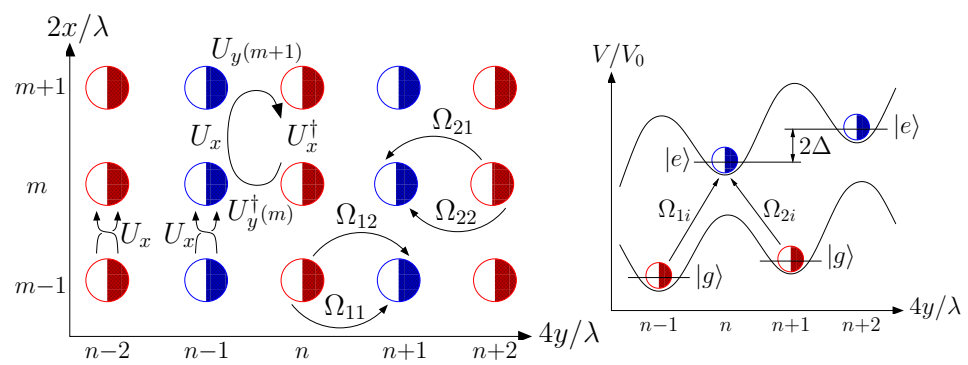

Figure 27. (Color online, see [135]). Optical lattice setup for U(2) gauge fields: Red and blue open semi-circles (closed semi-circles) denote atoms in states $\left|g_{1}\right\rangle$ and $\left|g_{2}\right\rangle$, respectively $\left(\left|e_{1}\right\rangle\right.$ and $\left|e_{2}\right\rangle$ ). Left) Hopping in the $x$-direction is laser assisted and allows for unitary exchange of colors; it is described by the same unitary hopping matrix $U_{x}$ for both $\left|g_{i}\right\rangle$ and $\left|e_{i}\right\rangle$ states. Hopping along the $y$-direction is also laser assisted and attains "spin dependent" phase factors. Right) Trapping potential in $y$-direction. Adjacent sites are set off by an energy $\Delta$ due to the lattice acceleration, or a static inhomogeneous electric field. The lasers $\Omega_{1 i}$ are resonant for transitions $\left|g_{1 i}\right\rangle \leftrightarrow\left|e_{2 i}\right\rangle$, while $\Omega_{2 i}$ are resonant for transitions between $\left|e_{1 i}\right\rangle \leftrightarrow\left|g_{2 i}\right\rangle$ due to the offset of the lattice sites. Because of the spatial dependence of $\Omega_{1,2}$ (running waves in $\pm x$ direction) the atoms hopping around the plaquette get the unitary transformation $U=U_{y}^{\dagger}(m) U_{x} U_{y}(m+1) U_{x}^{\dagger}$, where $U_{y}(m)=\exp \left(2 \pi i m \operatorname{diag}\left[\alpha_{1}, \alpha_{2}\right]\right)$, as indicated in the left figure (from [135]).

effect [577].

The method presented in Ref. [135] can be easily generalized to external gauge potentials of the form

$$
A_{i}(x, y)=a_{i}+b_{i}(x / a)+c_{i}(y / a)
$$

with $i=x, y$, and $a_{i}, b_{i}, c_{i}$ being essentially arbitrary $n \times n$ matrices. Furthermore, local disorder may be introduced in a controlled way that allows for small fluctuations of the matrices $a_{i}, b_{i}, c_{i}$. In particular, disorder can be made annealed, i.e. changing on a time scale comparable with the relevant time scales of the system, and, thus, mimics thermal fluctuations. It can be of significant amplitude, provided it does not drive the assisting lasers system out of resonance. Also, more complicated spatial dependences, e.g., piecewise linearity, of $\vec{A}$ are feasible by using static electric fields, laser induced potentials, etc. Additional lasers may introduce local, and in general time dependent unitaries. Such transformations would generate arbitrary local temporal components of the gauge potential, $A_{0}(x, y)$. Although for Yang-Mills fields in $(2+1) \mathrm{D}$, this component may be gauged out adapting the Weyl, or strict temporal gauge [578], the corresponding gauge transformations may introduce more complex spatial and temporal forms of the remaining two components of $\vec{A}$.

In the limit of weak fields, the continuum limit may be used, and the single particle Hamiltonian reduces to that of a particle in the gauge field corresponding to the above potential, i.e.:

$$
H=\frac{1}{2 m}\left[\left(p_{x}-A_{x}(x, y)\right)^{2}+\left(p_{y}-A_{y}(x, y)\right)^{2}\right] .
$$

The non-Abelian gauge fields and Hofstadter "moth" are interesting not only for 


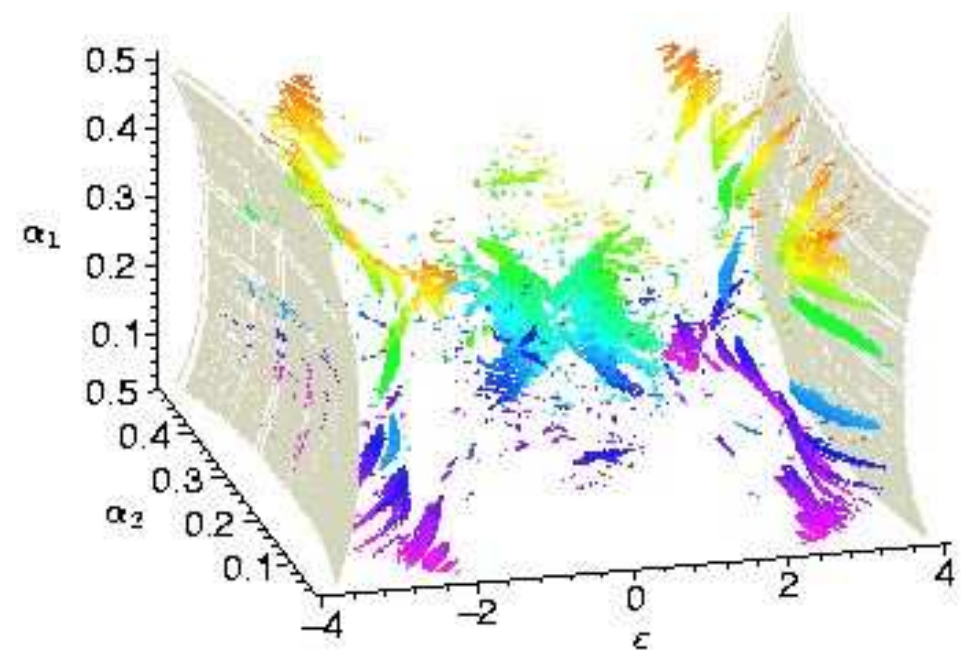

Figure 28. (Color online). The Hofstadter "moth" spectrum. Forbidden eigenenergies $\varepsilon$ are plotted versus $\alpha_{i}=p_{i} / q_{i}, \in[0,=0.5](i=1,2)$, where $q_{i} \leq 41$ and $\alpha_{1} \neq \alpha_{2}$ (from [135]).

fundamental reasons: they offer also a fascinating possibility of observing the nonAbelian Aharonov-Bohm effect and realizing non-Abelian atom interferometry and non-Abelian nonlinear atom optics $[135,579]$. Phase shifts in non-Abelian interferometers will correspond to matrices, and will depend not only on the perturbations of the trajectories of the interfering particles, but also on the specific locations of these perturbations.

Another fascinating possibility concerns the possibility of realizing novel FQHE states, that would be associated with fractional non-Abelian excitations. For instance, moving of a pseudo-hole around origin should induce a non-Abelian Berry "phase" described by a non-trivial matrix [580]. Particularly interesting in this context would be generalizations of methods used in [133]. The studies of non-Abelian FQHE in such fields has just began. The resulting model [174] is related to, yet very different from, the bilayer of FQHE with tunneling between the layers [442,570].

We expect that pseudo-hole in such model will transform according to the nonAbelian representations of the permutation group, or more precisely Artin's braid group, which in 2D considers particle exchange along the topologically distinct paths that avoid other particles (for a pedagogical review see [581], for recent discussion of wave functions of non-Abelions see [582]). There has been a long lasting quest for non-Abelian anyons in the condensed matter literature, but no clear experimental observation so far. The most prominent candidates are electronic (fermionic) $\nu=5 / 2$ FQHE state. This state has been observed in experiments, and Moore and Read [549] (see also [583]), and independently Greiter, Wen and Wilczek [584] proposed to explain it in terms of the "Pfaffian state" (see also Das Sarma and Pinczuk [585]). Recently, however, Töke and Jain [586] proposed an alternative "composite fermions" model of the $\nu=5 / 2$ state, which does not relate to nonAbelian statistics in any obvious manner. For bosons, a promising candidate is the 
$\nu=3 / 2$ state from the, so called, Read-Rezayi sequence of incompressible correlated liquids. This state seem to be a true ground state for the rapidly rotating gas of bosons interacting via contact (Van der Waals) forces with a moderate amount of dipolar interactions [550]. Such situation may be achieved, for instance, with Bose condensed Chromium, as in experiments of T. Pfau's group [145]. We hope that non-Abelian FQHE, due its profound and direct non-commutative character, will provide further, experimentally feasible examples of non-Abelian anyons. An alternative way to realize Pfaffian-like states and non-Abelions in 1D ultracold gases has been very recently proposed in Ref. [587].

\subsection{Ultracold gases and lattice gauge theories}

Another fascinating question concerns the possibility of using ultracold atoms for simulations of lattice gauge theories in $(2+1) \mathrm{D}$. The main difference is that in lattice gauge theories (LGT) gauge fields are dynamical variables, whereas they are obviously not in the scheme of Ref. [135]. Moreover, the scheme is realized in real rather than in imaginary time. Nevertheless, the big advantage of the proposal is that given a gauge field configuration, the dynamics of matter fields in real time are given for free. By generating various configurations of gauge fields, we may try to "mimic" the Monte Carlo sampling of LGT in the limit in which gauge fields affect the matter fields, but not vice versa. Averaging over both, annealed disorder and quantum fluctuations should approximate the statistical average in LGT. Such approach requires that generated configurations represent the characteristic or statistically relevant ones of corresponding LGT phases. For instance, configurations in the confinement sector should exhibit an area law fulfilled by Wilson loops, appropriate distributions of centre vortices, Abelian magnetic monopoles, instantons, merons, calorons, etc. (for a recent reviews, see [588,589]; see also [590]). Although the gauge fields accessible in the scheme of Ref. [135] are limited, at least some of them share characteristics with LGT phases.

The situation is much better when we turn to Abelian lattice gauge theories, since they reduce in some limits to models with ring exchange interactions, that involve product of operators over an elementary plaquette of the underlying lattice. The first proposal for such interactions has been formulated in Ref. [137]. The authors derived a spin model for a multicomponent Bose, or Fermi gas in the Mott limit with one particle per site. Due to the fact that in a triangular lattice tunneling from one site to another may occur directly, or via the remaining third site, they obtain 3-spin interactions in the third order of $t / U$ expansion. This proposal has the disadvantage that it involves very small energy scales and long time scales, since in the Mott limit $(t / U)^{3} \ll 1$.

Büchler et al. [43] consider a standard single component Bose-Hubbard model in a square (cubic) lattice coupled to lattice of diatomic "molecules", trapped in the centre of each plaquette (see Fig. 29) The coupling to the molecular state (of $d$ symmetry) takes the form:

$$
H_{m}=\nu \sum_{\text {plaquettes }} m_{\square}^{\dagger} m_{\square}+g \sum_{\text {plaquettes } \square}\left[m_{\square}^{\dagger}\left(b_{1} b_{3}-b_{2} b_{4}\right)+\text { h.c. }\right] .
$$

Two atoms may perform a Raman transition to a molecular state with the coupling 
(a)

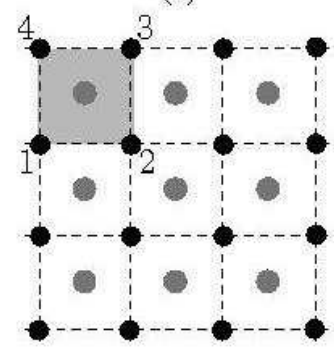

(b)

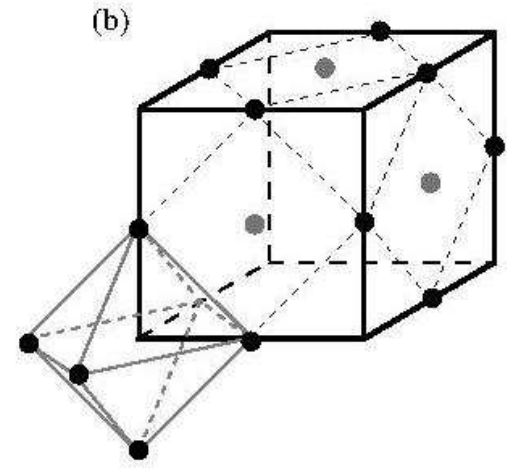

Figure 29. The scheme of realizing ring exchange interactions (redrawn from [43])

$g ; \nu$ denotes detuning of this transition. Perturbative elimination of the molecules leads to the effective ring exchange Hamiltonian for bosons:

$$
H_{R E}=K \sum_{\text {plaquettes } \square}\left(b_{1}^{\dagger} b_{2} b_{3}^{\dagger} b_{4}+b_{4}^{\dagger} b_{3} b_{2}^{\dagger} b_{1}-n_{1} n_{2}-n_{3} n_{4}\right) .
$$

More precisely, the model can be realised using atoms with two internal states: one state trapped in the square (cubic) lattice and described by the simple BoseHubbard model, and a second one trapped in the centres of the plaquettes in a site potential that is not symmetric, but rather has a point symmetry of the lattice. "Molecules" are build from two atoms in that second internal state, and have the $d-$ symmetry. The model described by the Bose-Hubbard Hamiltonian (Eq.(14)) plus $H_{m}$ is a promising candidate for a deconfined quantum critical point in 2D [591]. This is because it undergoes (most probably) a quantum phase transition between the bosonic superfluid phase (which occurs when $\nu \gg g$, and $t \gg g^{2} / \nu$, and which breaks the $U(1)$-symmetry), and a molecular "density wave" (stripes) phase (which occurs in the opposite limit, when $\nu<0$ and $|\nu| \gg t, g$, and breaks the translational symmetry). Both in $2 \mathrm{D}$ and $3 \mathrm{D}$ this model describes a $U(1)$ gauge theory, and is likely to exhibit a variety of quantum phases, such as $U(1)$ deconfined insulator, $U(1)$ deconfined phase, etc. [109,592].

Just before this review was submitted, Tewari et al. [138] proposed to use a dipolar Bose gas to simulate the compact $U(1)$ lattice gauge theory. Such model is well described by an extended Bose-Hubbard Hamiltonian, as discussed in [147]. Tewari et al. propose to use 2D kagomé, or 3D pyrochlore lattices, assuring isotropy of dipolar interactions between nearest neighbors on the lattice. In the limit in which the on-site interactions $U$ are comparable to the n.n. interactions $V$, and both are strong, the physics is dominated by the configurations where the fluctuations of the number of atoms in all elementary plaquettes is zero. In the lowest relevant order 
of perturbation theory $\left((t / U)^{3}\right)$ one obtains a hamiltonian with "ring exchange" term on a dual hexagonal lattice. This model reduces directly to the $U(1)$ lattice gauge theory, and allows to realize various fractionalized topological phases. Moreover, the authors propose methods of detecting signatures of the emergent $U(1)$ Coulomb phase, terming it as "emergence of artificial light in an optical lattice". It is finally worth stressing that by combining, vortex lines with fermions, one can realize perhaps the first connections of ultracold atoms with string theory [593].

\section{Quantum information with ultracold gases}

\subsection{Introduction}

There has recently been a real explosion of interest in the studies of the interface between quantum information (QI) and many body systems, both in condensed matter, as well as in the physics of ultracold atomic gases. First, in Ref. [31] a proposal of using ultracold atomic gases in optical lattices for QI was formulated. This has stimulated interest in distributed quantum information processing. Second, in Refs. [594-596], the first connections between entanglement and quantum phase transitions (QPT) were discussed. This discussion has opened fundamental questions of deeper understanding of QPT's as well as practical questions of employing QI ideas in simulation codes for many body quantum systems (see subsec. 3.10).

In this section we will (partially) review these two major themes of such studies. One of them uses realizable many body systems as viable systems for quantum computation and other quantum information tasks. In the other theme, the aim is to use quantum information concepts such as entanglement, to understand condensed matter and many body phenomena, like for instance quantum phase transitions.

This section has a slightly different character than the rest of the paper, in that it does not make distinction between condensed matter and atomic systems, but rather considers general properties of quantum states. It is also organized differently. In order to make the review self-contained, we begin with subsec. 8.2 by presenting necessary basic notions and tools of quantum information theory in general, and entanglement theory in particular. In the next subsection (subsec. 8.3 ), we consider quantum phase transitions from the quantum information perspective. In subsec. 8.4, we discuss possibilities of performing quantum computation using atoms in optical traps. The last subsection 8.5 is devoted to the concept of measurement-based quantum computation, both in ordered and disordered lattices.

\subsection{Entanglement: A formal definition and some preliminaries}

The concept of entanglement can be traced back to manuscripts of E. Schrödinger around 1932, when it was realised that the quantum mechanical formalism allows the existence of pure states of two systems, for which the information about the whole system is not the sum of the information about the separate systems. With the advent of quantum information, a more operational definition of entanglement was required. Such a definition was formulated by Werner in 1989 [597]. Suppose that there are two systems belonging to two observers called Alice (A) and Bob 
(B). They are, in principle, at distant locations, which, operationally means that they can only act on their local parts. Under such conditions, the most general state that they are able to prepare is of the form

$$
\varrho_{A} \otimes \varrho_{B}
$$

However, they may additionally be allowed to communicate classical information between them. In that case, the most general state that they are able to prepare is of the form

$$
\sum_{i} p_{i} \varrho_{A}^{i} \otimes \varrho_{B}^{i}
$$

where the $\left\{p_{i}\right\}$ are a set of probabilities. The set of operations that Alice and Bob are allowed to perform is then called local operations and classical communication (LOCC). The states that are of the form in Eq. (111) are called separable states, and they (and only they) can be prepared under LOCC only. However, there are states that cannot be written in the form in Eq. (111), and consequently it is not possible to prepare them by LOCC, an example being the singlet state. These states are called entangled states.

A pure state $|\psi\rangle_{A B}$ is separable if and only if its local subsystems are also pure, that is when it can be written as $\left|\psi_{1}\right\rangle_{A} \otimes\left|\psi_{2}\right\rangle_{B}$. The case of mixed states is, however, nontrivial. In fact, so far an operational necessary and sufficient criterion for detecting entanglement in mixed states has not been found (see [598] for a tutorial). Using a semidefinite programming, it has been shown that separability can be tested in a finite number of steps, although there is no limit on the number of steps needed [599]. See also Ref. [600]. However, there exist many efficient criteria, that are either necessary or sufficient. One of them is described below.

8.2.1 The partial transposition criterion for detecting entanglement. The partial transposition criterion is a very useful criterion for detecting entanglement. We will require the notion of partial transposition of a density matrix $\varrho_{A B}$. If partial transposition of $\varrho_{A B}$ is taken over the subsystem $A$, denoted by $\varrho_{A B}^{T_{A}}$, then its elements are defined as

$$
\left(\rho_{A B}^{T_{A}}\right)_{i \mu, j \nu}=\left(\rho_{A B}\right)_{j \mu, i \nu}
$$

where the subscripts $i, j$ are for Alice's subsystem and the subscripts $\mu, \nu$ are for Bob's subsystem. Note that although the partial transposition depends upon the choice of the basis in which $\varrho_{A B}$ is written, the eigenvalues do not depend on the basis. We say that a state has positive partial transposition (PPT) whenever $\varrho^{T_{A}} \geq 0$, i.e. all the eigenvalues of $\varrho^{T_{A}}$ are nonnegative. Otherwise the state has negative partial transposition (NPT). Peres [601] noticed that for separable states, the eigenvalues of the partially transposed density matrix are always positive. That is, all separable states are PPT, and there exist entangled states which are NPT. Failing to be PPT is thus a signature of entanglement. An NPT state is often said to violate the PPT criterion. One of the most important results of the theory of entanglement has been proven by the Horodecki family, who showed that in $\mathbb{C}^{2} \otimes \mathbb{C}^{2}$ and $\mathbb{C}^{2} \otimes \mathbb{C}^{3}$, a state is separable if and only if it is PPT [602]. This is no longer true in higher dimensions. 
8.2.2 Entanglement measures. The partial transposition criterion provides a tool to check whether the given state is entangled or not. Now, we will discuss ways to find out how much entanglement a given state has, once we know that it is entangled. This quantification is necessary, at least partly because entanglement is viewed as a resource in quantum information theory. There are several complementary ways to quantify entanglement (see [603-612] and references therein). We will present here three possible ways to do so.

Entanglement of formation. Consider a bipartite pure state $\left|\psi_{A B}\right\rangle$ shared between Alice and Bob. It was shown by Bennett et al. [613], that given $n E\left(\psi_{A B}\right)$ copies of the singlet state shared between Alice and Bob, they can by LOCC, transform them into $n$ copies of the state $\left|\psi_{A B}\right\rangle$, if $n$ is large, where:

$$
E\left(\psi_{A B}\right)=S\left(\varrho_{A}\right)=S\left(\varrho_{B}\right)
$$

with $\varrho_{A}$ and $\varrho_{B}$ being the local density matrices of $\left|\psi_{A B}\right\rangle$. Here $S(\rho)$ is the von Neumann entropy of $\rho$, given by:

$$
S(\rho)=-\operatorname{tr} \rho \log _{2} \rho .
$$

If the state $\left|\psi_{A B}\right\rangle$ describes a $\mathbb{C}^{d_{1}} \otimes \mathbb{C}^{d_{2}}$ system, then $E$ ranges from 0 to $\log _{2} d$, where $d=\min \left\{d_{1}, d_{2}\right\}$. Note that $E$ is vanishing for separable (pure) states. A state is called maximally entangled in $\mathbb{C}^{d_{1}} \otimes \mathbb{C}^{d_{2}}$, if its $E$ is $\log _{2} d$. The singlet state is a maximally entangled state in $\mathbb{C}^{2} \otimes \mathbb{C}^{2}$ and its entanglement is unity. This amount of entanglement of a singlet is also called an ebit. A maximally entangled state in $\mathbb{C}^{d_{1}} \otimes \mathbb{C}^{d_{2}}$ has $\log _{2} d$ ebits. An example of a maximally entangled state in $\mathbb{C}^{d_{1}} \otimes \mathbb{C}^{d_{2}}$ is:

$$
\frac{1}{\sqrt{d}} \sum_{i=1}^{d}\left|\alpha_{i}\right\rangle_{A} \otimes\left|\beta_{i}\right\rangle_{B},
$$

where $\left|\alpha_{i}\right\rangle_{A}\left(\left|\beta_{i}\right\rangle_{B}\right)$ are orthogonal states in Alice's (Bob's) subsystem. With the above terminology, we can therefore say that the state $\left|\psi_{A B}\right\rangle$ has $E\left(\psi_{A B}\right)$ ebits. Since $E\left(\psi_{A B}\right)$ is the number of singlets required to prepare a copy of the state $\left|\psi_{A B}\right\rangle$, it is called the "entanglement of formation" of $\left|\psi_{A B}\right\rangle$. We are therefore using the amount of entanglement of the singlet state as our unit in quantifying entanglement.

For (bipartite) pure states, the entanglement of formation is essentially the only (asymptotic) measure of entanglement, as the singlet state can be locally obtained from a pure state at the same asymptotic rate [613].

After obtaining the definition of entanglement of formation for pure states, let us now define this measure for mixed states [603]. Any mixed state can be expressed as a mixture (convex combination) of pure states. That is, a mixed state $\varrho$ can always be expressed as:

$$
\varrho=\sum_{i} p_{i}\left|\psi_{i}\right\rangle\left\langle\psi_{i}\right|,
$$

where $\left\{p_{i}\right\}$ are a set of probabilities. The states $\left|\psi_{i}\right\rangle$ are not necessarily orthogonal. 
It is then tempting to define the entanglement of formation of a mixed state

$$
\varrho_{A B}=\sum_{i} p_{i}\left|\psi_{A B}^{i}\right\rangle\left\langle\psi_{A B}^{i}\right|
$$

as the average

$$
\sum_{i} p_{i} E\left(\psi^{i}\right)
$$

However, a mixed state can be decomposed into pure states in an infinite number of ways. The entanglement of formation $E_{F}$ of $\varrho_{A B}$ is defined as the minimum over all such averages. Precisely, [603]:

$$
E_{F}\left(\varrho_{A B}\right)=\min \sum_{i} p_{i} E\left(\psi_{A B}^{i}\right)
$$

where the minimum is over all decompositions of $\varrho_{A B}$ into pure states.

The procedure for calculating entanglement of formation of any state in $\mathbb{C}^{2} \otimes \mathbb{C}^{2}$ was given in Refs. [614,615]. For states in higher dimensions, the entanglement of formation (or even more difficult asymptotic entanglement cost for many copies) has been calculated for only a few rare instances using certain symmetries (see for instance Ref. [616-620]).

Concurrence. As stated before, the entanglement of formation of an arbitrary (possibly, mixed) state in $\mathbb{C}^{2} \otimes \mathbb{C}^{2}$ has been calculated. It is given by:

$$
E_{F}\left(\varrho_{A B}\right)=\mathrm{H}\left(\frac{1+\sqrt{1-C^{2}}}{2}\right),
$$

where

$$
C=C(\varrho)=\max \left\{0, \lambda_{1}-\lambda_{2}-\lambda_{3}-\lambda_{4}\right\},
$$

and $H(\cdot)$ is the binary entropy function, defined for $0 \leq x \leq 1$ as $H(x)=-x \log _{2} x-$ $(1-x) \log _{2}(1-x)$. The $\lambda_{i}$ are the eigenvalues (with $\lambda_{1}$ being the greatest) of the Hermitian matrix $\left(\varrho^{\frac{1}{2}} \varrho \varrho^{\frac{1}{2}}\right)^{\frac{1}{2}}$, where $\tilde{\varrho}=\sigma_{y} \otimes \sigma_{y} \varrho^{*} \sigma_{y} \otimes \sigma_{y}$, with the complex conjugation over $\varrho$ being taken in the $\sigma_{z}$ eigenbasis.

In $\mathbb{C}^{2} \otimes \mathbb{C}^{2}$, the quantity $C\left(\varrho_{A B}\right)$, called the concurrence, is defined for arbitrary states, and moreover, the entanglement of formation and concurrence are monotonically nondecreasing functions of each other, with both quantities ranging from 0 to 1 . Therefore, in $\mathbb{C}^{2} \otimes \mathbb{C}^{2}$, we will also use the concurrence as a measure of entanglement.

Logarithmic negativity. The partial transposition criterion can be used to define a useful measure of entanglement, that is easily computable. To define the measure, let us first introduce a quantity called negativity. The negativity $N\left(\varrho_{A B}\right)$ of a bipartite state $\varrho_{A B}$ is defined as the absolute value of the sum of the negative eigenvalues of $\varrho_{A B}^{T_{A}}$. The logarithmic negativity (LN) is defined as [621]

$$
E_{N}\left(\varrho_{A B}\right)=\log _{2}\left(2 N\left(\varrho_{A B}\right)+1\right) .
$$


Note that for two qubit states, $\varrho_{A B}^{T_{A}}$ has at most one negative eigenvalue [622]. Moreover, it follows from our discussion in 8.2.1 that for states in $\mathbb{C}^{2} \otimes \mathbb{C}^{2}$ and in $\mathbb{C}^{2} \otimes \mathbb{C}^{3}$, a positive LN implies that the state is entangled, while $E_{N}=0$ implies that the state is separable.

The logarithmic negativity has an operational interpretation in terms of another measure related to the entanglement of formation [623], and moreover, is an entanglement monotone under deterministic LOCC [624]. However, it is not convex.

\subsection{Entanglement and phase transitions}

Over the past few years, the question whether quantum phase transitions (QPT) can be understood from the perspective of quantum information has been posed, and at least partially positively answered.

QPTs are nonanalyticities in the ground state energy of physical systems. A typical situation is the following (see e.g. [154]). Consider a physical system that is defined on some lattice, and that is described by a Hamiltonian of the form

$$
H=H_{0}+\lambda H_{1},
$$

where $\left[H_{0}, H_{1}\right] \neq 0$. The parameter $\lambda$ is to be visualized as an external parameter, which can be changed by the experimenter. In the limit of an infinite lattice, if as the system crosses a certain value of $\lambda=\lambda_{c}$, the ground state has a qualitative change, and there is an associated nonanalyticity in the ground state energy at $\lambda=\lambda_{c}$, then the system is said to have a QPT at that point. Since the transition is in the ground state, so that the system is at zero temperature, the transition is driven by quantum fluctuations (and not by thermal fluctuations like in classical phase transitions), and this is the reason why it is called a "quantum" phase transition.

The study of entanglement in strongly correlated systems was initiated in e.g. [625-644] (and references therein). It was realized by Osborne and Nielsen [594,595] and Osterloh et al. [596] that the distinctly quantum phenomenon of QPTs can be related to the quantum phenomenon of entanglement. The scaling of entanglement as an infinite chain of spin-half particles, described by the XY model, goes through a QPT was considered. Ideally, one would like to consider the scaling of the multiparticle entanglement of the whole infinite spin system in the ground state. However, the subject of multiparticle entanglement is not fully developed at present. As a way out, the authors in Refs. [594-596] considered the nearest neighbor entanglement of the ground state of the spin chain. This required the consideration of entanglement of (mixed) states in $\mathbb{C}^{2} \otimes \mathbb{C}^{2}$, which is relatively well-developed. We consider this topic in subsection 8.3.1.

As we just commented, for higher number of parties, the theory of entanglement is not so well developed, even for pure states, at least not quantitatively. However, the entanglement of pure states in arbitrary bipartite dimensions is quite wellunderstood, as we have discussed in 8.2.2. Consequently, and since the ground state is a pure state, one may consider the scaling of entanglement of the ground state for a bipartite split consisting on a certain number of sites $n$ and the rest of the lattice, as $n$ grows. The entanglement of one site to the rest of the chain was considered in e.g. Ref. [595,638,639], and the scaling in this scenario was initiated by Vidal et al. [298]. We discuss this topic in 8.3.2. 
Yet another type of scaling was considered by Verstraete et al. [302] by using the concept of "localizable entanglement", which will be defined and then its scaling discussed in 8.3.3.

We omit several topics in the following, such as the topic of scaling of certain multipartite entanglement measures (see e.g. [645-649], and references therein). There is also an interesting string of research that deals with criticalities in the Hubbard model (see e.g. [646,650-652], and references therein). Dorner et al. [65] consider a string of neutral atoms in a one dimensional beam splitter configuration, where the longitudinal motion is controlled by a moving optical lattice potential; they show that it is possible to create maximally entangled states in this setup, by crossing a QPT. Lambert et al. [653] considers an infinite collection of twolevel atoms interacting via the Dicke Hamiltonian with a single bosonic mode, and describes the behaviour of entanglement when the system is near its QPT.

Most of the papers that deal with the scaling of entanglement near criticality deal with asymptotic measures of entanglement, or those related to such measures. The entanglement of formation (and concurrence), for example, is defined in terms of the the entropy of the reduced density matrix of a pure state, which has meaning only in the asymptotic regime. Exceptions include the work by Eisert and Cramer [654], which considers single copy entanglement of the ground state of critical quantum spin chains. In the last part of this subsection, a possible way to use (bipartite) entanglement to observe a type of phase transitions (called dynamical phase transitions) in the evolved state of a strongly correlated system is discussed [655].

8.3.1 Scaling of entanglement in the reduced density matrix. The first system for which the scaling of entanglement near a QPT was considered is a system of spin-half particles on a chain, described by the XY Hamiltonian:

$$
H_{X Y}=-\frac{J}{2} \sum_{i}\left[(1-\gamma) \sigma_{i}^{x} \sigma_{i+1}^{x}+(1+\gamma) \sigma_{i}^{y} \sigma_{i+1}^{y}\right]-h \sum_{i} \sigma_{i}^{z},
$$

with $J>0, h>0$, and $0<\gamma \leq 1$. This system is known to undergo a QPT as the parameter $\lambda=\frac{J}{2 h}$ passes over $\lambda=\lambda_{c} \equiv 1$ (see e.g. [154,656-662]).

In Refs. [594-596], it was proposed that this QPT may be analysed by looking at the behavior of the entanglement in the two site states (the state obtained by tracing over all except two sites of the chain) of the ground state of the system, as it goes through the QPT (see Fig. 30). In particular, in the case of the Ising Hamiltonian $(\gamma=1)$ on an infinite chain, the $\lambda$-derivative of the nearest neighbor concurrence diverges as [596]:

$$
\partial_{\lambda} C_{1}=\frac{8}{3 \pi^{2}} \ln \left|\lambda-\lambda_{c}\right|,
$$

(up to an additive constant) as $\lambda$ approaches $\lambda_{c}$ (See Fig. 30). The subindex 1 of $C_{1}$ indicates the fact that the nearest neighbor entanglement is calculated.

The Refs. [594-596] stimulated further studies of two-site entanglement to characterise QPTs. Bose and Chattopadhyay [640] have considered it in the case of some frustrated spin models in one dimension, ladder, and two dimensions. Two-site entanglement in the XXZ chain was studied by Gu et al. [664]. The Lipkin-Meshkov- 


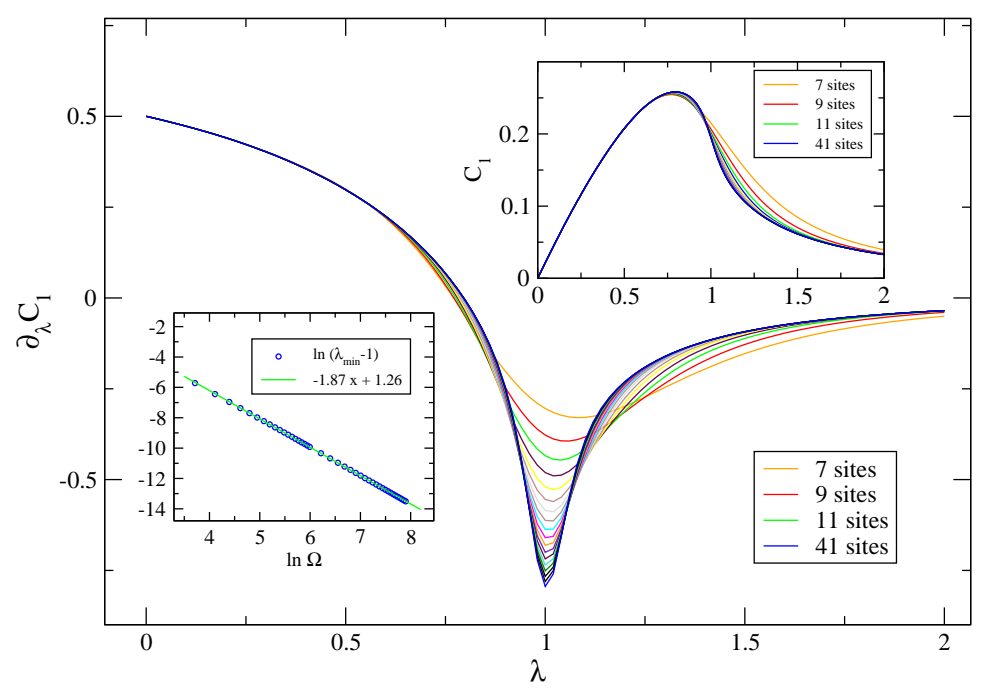

Figure 30. (Color online).Scaling of nearest neighbour entanglement of the ground state of the transverse Ising Hamiltonian on a chain (from [663]). The inset at top right shows the nearest neighbour entanglement, while the main figure shows its derivative, plotted against the parameter $\lambda$. In the main figure (top right inset), say at $\lambda=1$ (say at $\lambda=1.25$ ), the curves from the top are respectively for $7,9,11, \ldots, 41$ sites (the quality of the figure improves in the online version).

Bottom left inset: The position $\lambda_{\min }$ of the minimum of $\partial_{\lambda} C_{1}$ in the main figure changes with the total number of sites. The current inset shows the plot of the logarithm of $\lambda_{\min }-1$ against the logarithm of the total number of sites (denoted in this figure as $\ln \Omega$ ).

Glick model has been considered in Refs. [665-667]. Stauber and Guinea [668] have looked at the entanglement of spins at the boundary of a spin chain governed by the transverse Ising model, as well as that of two spins coupled to a critical reservoir. The anisotropic ferromagnetic Heisenberg chain in the presence of domain walls was considered by Alcarez et al. [669].

Yang [670] obtained the following apparently counterintuitive result. An exactly solvable three-spin interaction Hamiltonian was considered, and it was shown that there can appear a discontinuity of the first derivative of the two-site concurrence, which has no connection to any QPT (see also Refs. [665,671]). It was further shown that this discontinuity originates from finding the maximum in the definition of concurrence. Subsequently, Wu et al. [672], found necessary and sufficient conditions under which discontinuities in the two-site entanglement (as quantified by concurrence, or negativity) can be an indicator of a QPT in physical systems governed by Hamiltonians containing only two-body interactions.

8.3.2 Entanglement entropy: Scaling of spin block entanglement. A different approach than the two-site entanglement was considered by Vidal et al. [298]. The idea is to consider a block of spins and consider its entanglement with the rest of the system, for the ground state, as the block size increases. Since the ground state is a pure state, a good measure of entanglement of a block of spins with the rest of the system, is the entropy of the block of spins. This has been termed 
the entanglement entropy (EE) of the model. The models that were considered in Ref. [298] (see also [673]) are the one dimensional XY and XXZ models, where the latter is described by the following Hamiltonian:

$$
H_{X X Z}=\sum_{i}\left(\sigma_{i}^{x} \sigma_{i+1}^{x}+\sigma_{i}^{y} \sigma_{i+1}^{y}+\Delta \sigma_{i}^{z} \sigma_{i+1}^{z}-\lambda \sigma_{i}^{z}\right)
$$

It was shown that away from the criticalities, EE saturates to a constant as a function of the block size. However, near the criticalities, EE diverges logarithmically with block size. Similar behaviour was seen to be true for the Affleck-KennedyLieb-Tasaki valence bond solid models by Fan et al. [674].

Techniques from conformal field theory were used by Korepin [650] for evaluating the EE in the limit of large block size for infinite spin chains. Its et al. [675] have evaluated this limiting EE for an infinite chain governed by the XY model.

EE was also considered by Wellard and Orús [676] for the case of QPTs in antiferromagnetic (Ising) planar cubic lattices in the presence of homogeneous transverse and longitudinal magnetic fields, and by Latorre et al. Ref. [677] for the LipkinMeshkov-Glick model.

The relation between entanglement entropy and area for the cubic harmonic lattice has been discussed in Refs. [678,679] (for fermionic systems, see [680,681]) (cf. $[314,682])$.

In Ref. [683], an unbounded increase of entanglement entropy was obtained for a lattice of spins that interact via some long-range Ising type interaction.

8.3.3 Localizable entanglement and its scaling. For a given multiparty state, $\varrho_{1,2, \ldots, N}$, of $N$ parties, the localizable entanglement (LE) [302] is the maximum average entanglement that can be made to be shared between two predetermined parties (say, 1 and 2), by measurements at the rest of the parties. We still have to state which entanglement measure we consider in finding the average entanglement. Similar considerations were used in the context of violation of Bell inequalities [684] in Refs. [685-687]. In Ref. [302], the LE of the ground state of certain spin-half systems were considered, where concurrence was used as the measure of entanglement in the definition of average entanglement.

Using the concept of LE, a related "entanglement length", $\xi_{E}$, was defined in [301], which gives the typical length scale (of a spin-half chain, e.g.) at which it is possible to create maximally entangled states by performing measurements at the rest of the parties. Let us make the definition more specific in the case of a spin chain. Let $E_{i, i+n}$ be the LE that can be made to share between the $i$ th and the $(i+n)$ th spins of the chain. If $E_{i, i+n}$ decays exponentially with $n$, then the entanglement length of the chain is defined by:

$$
\xi_{E}^{-1}=\lim _{n \rightarrow \infty}\left(\frac{-\ln E_{i, i+n}}{n}\right) .
$$

If the decay of $E_{i, i+n}$ is non-exponential, then the entanglement length is infinite. A similar definition was given by Aharonov [688], considering a transition from quantum to classical physics. The definition of entanglement length parallels that 
of correlation length, for which if the correlation $\left\langle O_{i} O_{i+n}\right\rangle$ between two observables $O_{i}$ and $O_{i+n}$ decays exponentially, then the correlation length $\xi$ is defined by:

$$
\xi^{-1}=\lim _{n \rightarrow \infty}\left(\frac{-\ln \left\langle O_{i} O_{i+n}\right\rangle}{n}\right) .
$$

A diverging correlation length implies a diverging entanglement length. However, the converse is not true: for example, the gapped spin system governed by the modified Affleck-Kennedy-Lieb-Tasaki model, which has a finite correlation length, was proven to have a diverging entanglement length by Verstraete et al. [301].

8.3.4 Critical behaviour in the evolved state. Phase transitions are usually viewed for ground states, at least when considered from the perspective of quantum information theory. In contrast, Ref. [655] considers a criticality of nearest-neighbor entanglement in the evolution of an infinite spin chain described by the asymmetric $X Y$ model in a time-dependent transverse field.

The initial state of the evolution is taken to be the canonical equilibrium state at zero temperature (which, in this case, is the ground state). The transverse field is then suddenly turn off at time equal to zero. The system is thus initially disturbed, and its properties are then studied at later times. The nearest-neighbor entanglement (quantified by the logarithmic negativity) in the evolved state at a fixed time shows a criticality (which has been called a dynamical phase transition (DPT)) with respect to the transverse external field. The region of the initial transverse field for which the entanglement is nonvanishing (vanishing), at a fixed time, is referred to as the "entangled phase" ("separable phase") (see Fig. 31).

Interestingly, the nature of the DPT depends on whether we are near, or far from the time of initial disturbance. Moreover, for values of the initial transverse field near the criticalities, as well as in the separable phase, and for short times, the nearest-neighbor entanglement shows nonmonotonicity with respect to temperature. Accordingly, the criticalities are referred to as "critical regions", signifying that "critical" effects persist for a small region around the critical value of the transverse field.

The study of nonmonotonicity of entanglement with respect to temperature is interesting, as preservation of entanglement in a hostile environment is one of the main challenges in quantum computation and quantum information in general. A common belief is that temperature is a form of noise, i.e. it destroys the subtle quantum correlations. However, this seems not to be universally true.

Nonmonotonicity of entanglement with respect to temperature was also found by Arnesen et al. [632], in the canonical equilibrium state of the one-dimensional isotropic antiferromagnetic Heisenberg model. Similar behavior was obtained by Scheel et al. [689], in the Jaynes-Cummings model. The idea that dissipation can assist generation of entanglement has also been put forward in Refs. [690-696].

It should be mentioned here that the asymptotics of temperature correlations in the isotropic (i.e $\gamma=0) X Y$ model was considered by Its et al. [697] (see also [698-701]). Also, previous studies of the quantum dynamics of spin models after a rapid change of the field include Refs. [32,657,659-662,702,703], while effects of a sudden switching of the interaction in arrays of oscillators were studied in 


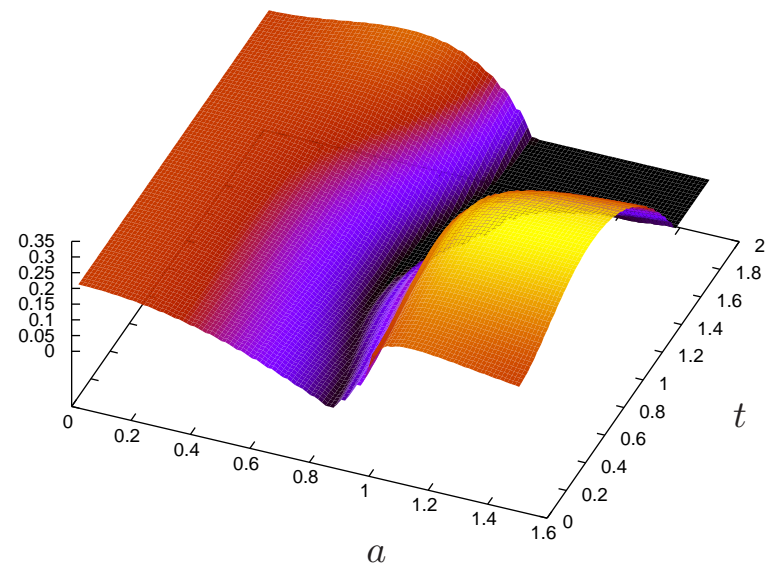

Figure 31. Dynamical phase transitions: a "river" of separable states between the two regions of control parameter space where nearest neighbour entaglement is non-zero in an asymmetric XY model in a time-dependent transverse field. (from [655]). Entanglement (logarithmic negativity) is plotted against the initial transverse field $(a)$ and (real) time $(t)$. A similar behaviour is absent in magnetization [655].

Ref. [704].

\subsection{Quantum computing with lattice gases}

The computers that have become a part of our daily life are very efficient and useful machines. However, there are certain problems that may not be efficiently solved with such computers. For example, it is believed that the problem of finding prime factors of a given integer is exponentially inefficient on a classical computer, since the number of steps required to find the factors scales exponentially with the size of the input integer.

Classical computers follow the principles of classical physics, but it is possible to consider computers that are ruled by the laws of quantum mechanics. There are at least two important motivations for considering computing at a quantum level: (1) The classical laws of physics governing the functioning of classical computers are an approximation that suits our purpose for the current size of microchips. If size is going to keep decreasing, so that computational speed increases, quantum effects will unavoidably emerge; (2) In 1994, Shor discovered a quantum algorithm that can efficiently factorize integers into their prime factors [23].

There are several proposals for implementing quantum computing [705]. A very promising one was put forward by Cirac and Zoller in 1995 that uses cold trapped ions interacting with laser beams [24]. For the effects of environmental decoherence on such an ion trap quantum computer and possible ways to get over them, see e.g. [706-711], and references therein. Following the Cirac-Zoller proposal, a fun- 
a)

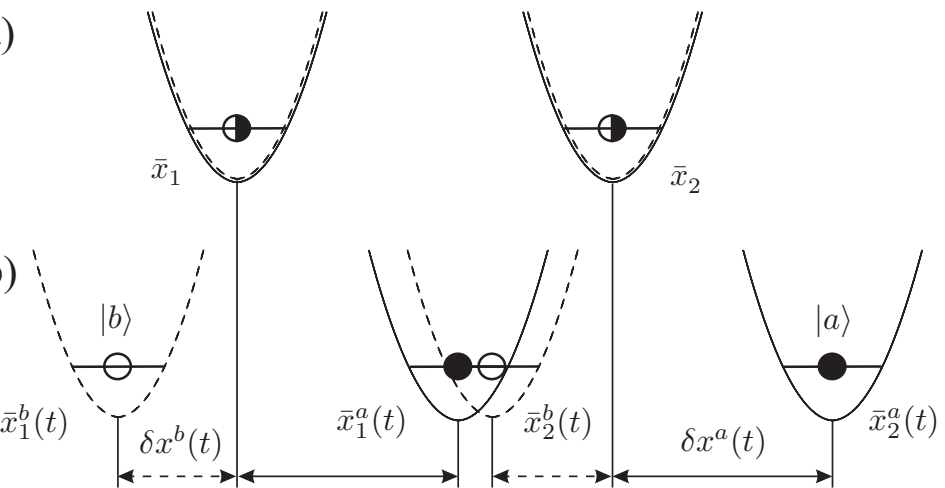

Figure 32. Schematic representation of the gate operation. Solid and dashed lines represent potentials felt by the atoms in the different internal states, respectively. By shifting the potentials, the pair of atoms in the middle experiences a collisional shift (from [31])

damental quantum logic gate, the CNOT gate, was experimentally demonstrated by Monroe et al. [712], where the two qubit state was encoded in the two internal states and two external states of a single trapped ion. For recent developments in ion trap computers, see [713-717]. In particular, Ref. [714] has realized the CNOT gate by two ${ }^{40} \mathrm{Ca}^{+}$ions, held in a linear Paul trap. Jaksch et al. [31] proposed to use atoms in an optical lattice for quantum computation. They use cold controlled collisions between two atoms in moving trap potentials for implementing the following two-qubit gate:

$$
\begin{aligned}
|a\rangle_{1}|a\rangle_{2} & \rightarrow|a\rangle_{1}|a\rangle_{2}, \\
|a\rangle_{1}|b\rangle_{2} & \rightarrow e^{-i \phi_{a b}}|a\rangle_{1}|b\rangle_{2}, \\
|b\rangle_{1}|a\rangle_{2} & \rightarrow|b\rangle_{1}|a\rangle_{2}, \\
|b\rangle_{1}|b\rangle_{2} & \rightarrow|b\rangle_{1}|b\rangle_{2} .
\end{aligned}
$$

The two qubits are encoded in the internal states $|a\rangle$ and $|b\rangle$ of the two atoms. The two atoms are labelled as 1 and 2 and each atom is trapped in the ground state of a potential well. The phase $\phi_{a b}$ will be a fixed number depending on the implementation. Initially, the potential wells are at a distance that is sufficiently large distance so that there is no interaction between the atoms. They are then moved along certain trajectories toward each other, so that the atoms interact for a certain time, and then finally the wells are restored to their initial positions. The potential wells are moved adiabatically, so that the atoms remain in the respective ground states of the wells. The implementation requires an interaction that induces internal state dependent potentials, so that the atoms in different internal states feel different potentials (see Fig. 32).

The effective Hamiltonian governing the system is given by:

$$
H=\sum_{i}\left[\omega^{a}(t) a_{i}^{\dagger} a_{i}+\omega^{b}(t) b_{i}^{\dagger} b_{i}+u^{a a}(t) a_{i}^{\dagger} a_{i}^{\dagger} a_{i} a_{i}+u^{b b}(t) b_{i}^{\dagger} b_{i}^{\dagger} b_{i} b_{i}\right]
$$




$$
+\sum_{i j} u_{i j}^{a b}(t) a_{i}^{\dagger} a_{i} b_{j}^{\dagger} b_{j}
$$

$a_{i}\left(a_{i}^{\dagger}\right)$ and $b_{i}\left(b_{i}^{\dagger}\right)$ are the annihilation (creation) operators of the internal levels $|a\rangle$ and $|b\rangle$ for an atom in the ground state of the potential well at position $i$. By assuming that (i) there is no sloshing motion of the atoms in the wells when the latter are moved, (ii) the potentials are moved so that the atoms remain in the ground state (adiabaticity condition), and (iii) the rms velocity of the atoms in the vibrational ground state is sufficiently small so that zero energy $s$-wave scattering approximation is valid, then it is possible to show that the internal states of the atoms transform in the way described in Eq. (114).

There are several other papers that deal with quantum computation with atoms in optical lattices. In particular, Brennen et al. [718] have used dipole-dipole interactions to implement the CNOT gate. For further work, see e.g. $[29,32,64,65,160$, $162,369,719-742]$.

\subsection{Generation of entanglement: The one-way quantum computer}

There has been several studies for generating entanglement in different physical systems. We want to discuss generation of entanglement from the perspective of performing quantum computation. We shall discuss entanglement generation in ordered and disordered lattices separately.

8.5.1 The one-way quantum computer. The one-way quantum computer (also referred to as "measurement-based quantum computation") was proposed by Raussendorf and Briegel [743], who showed that arbitrary quantum computation can be simulated via single-particle measurements on a specially prepared quantum state termed as "cluster state". Cluster states can be created efficiently in any ultracold atomic systems with Ising-type interactions, and allow the possibility of preparing the system in a specific initial product state. A proposal to implement cluster states uses four atomic levels trapped in appropriately tuned optical lattices [744] (cf. [745, 746]).

To define cluster states [747], consider an arbitrary graph made up of $N$ "vertices" (which will be identified with the qubits making up the quantum computer), and a certain number of "edges" (which will be identified with Ising interactions between the qubits) connecting the vertices (see Fig. 33). The terminology used here is

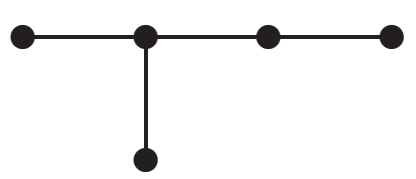

Figure 33. An example of a graph. In this case, it has five vertices and four edges. The vertices represent qubits, and the edges represent Ising interactions between them.

from graph theory (see e.g. [748]), and thereby cluster states are also called "graph 
states". Suppose that the qubits are spin-half particles, and that each of them is prepared in the state $|+\rangle=(|0\rangle+|1\rangle) / \sqrt{2}$, where $|0\rangle$ and $|1\rangle$ are respectively the spin up and down states in the $z$ direction. We then apply an Ising interaction governed by the Hamiltonian

$$
H=-J \sum \sigma_{i}^{z} \sigma_{j}^{z}
$$

between the qubits, where the summation runs over all pairs $(i, j)$ of sites that are connected by an edge in the graph. After a certain finite time $\left(=\frac{\pi \hbar}{4 J}\right)$, the state

$$
\left|\phi_{C}\right\rangle=\prod C S_{i j}|+\rangle^{\otimes N}
$$

is created. Here $C S_{i j}$ is the controlled-PHASE gate, defined as

$$
\begin{aligned}
& |0\rangle|0\rangle \rightarrow|0\rangle|0\rangle, \\
& |0\rangle|1\rangle \rightarrow|0\rangle|1\rangle, \\
& |1\rangle|0\rangle \rightarrow|1\rangle|0\rangle, \\
& |1\rangle|1\rangle \rightarrow i|1\rangle|1\rangle,
\end{aligned}
$$

acting on the spins $i$ and $j$. The product on the right hand side of Eq. (115), again runs over all pairs $(i, j)$ of sites that are connected by an edge in the graph. The state $\left|\phi_{C}\right\rangle$ is called the cluster state corresponding to the given graph. These states have several interesting properties. Perhaps the most interesting property is that for sufficiently large graphs, the corresponding cluster states can be used as a substrate for quantum computational tasks. More specifically, any quantum gate can be implemented with this resource, simply by performing measurements on the different spins of a suitably chosen graph, where it is assumed that the choice of a measurement basis on a particular spin may depend on outcomes of previous measurements on other spins. As an example, the Hadamard gate $(\mathbb{H})$, defined as

$$
\begin{aligned}
|0\rangle & \rightarrow(|0\rangle+|1\rangle) / \sqrt{2}, \\
|1\rangle & \rightarrow(|0\rangle-|1\rangle) / \sqrt{2},
\end{aligned}
$$

can be implemented in the following way. Suppose that at a certain point of computation, we obtain the state $\left|\psi_{i n}\right\rangle$, which we want to transform into $\mathbb{H}\left|\psi_{i n}\right\rangle$. This can be realized by using a graph of five vertices in a chain as shown in Fig. $34[743,749,750]$. The input state is initially at the extreme left. The rest of the spins are initially in the state $|+\rangle, \sigma_{z}|+\rangle=|+\rangle$. Ising interaction with nearest neighbor interactions (as depicted in the figure by the edges) for the requisite amount of time (equivalent to the controlled-PHASE gates, as described in the text) results in a (entangled) state of the five spins. Subsequently, measurements are performed on all spins except that on the extreme right, in the eigenbasis of the operator indicated over the corresponding vertices. This results in the state $\mathbb{H}\left|\psi_{\text {in }}\right\rangle$ at the extreme right spin. A five-spin graph and corresponding measurements implementing an arbitrary single-qubit gate, and a 15-spin graph implementing the CNOT gate is given in the same papers. 


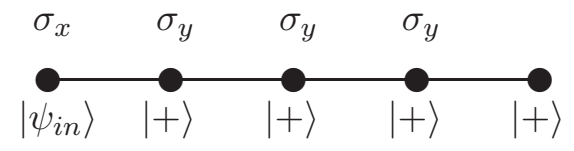

Figure 34. A graph to implement the Hadamard gate.

There is a large body of work in this direction that deal with several important issues ( see e.g. [744,751-774]). In particular, Dür et al. [683] deals with the entanglement properties of the time-evolved states in a model with long-range Ising-type interactions, in the thermodynamic limit.

8.5.2 Disordered lattice. Interestingly, it is also possible to perform quantum computing in systems with quenched disorder. This may sound contradictory, but we want to employ here the possibility of creating controlled disorder in atomic gases in optical lattices. Here, by "controlled", we mean that a particular realization of disorder remains fixed for times much longer than over which we monitor the evolution of the system (the disorder is "quenched"). This allows, as discussed in Section 4, to study Anderson and Bose glasses in a Bose gas [68], or spin glasses with short range interactions in Fermi-Bose, or Bose-Bose mixtures [86]. Using linear chains of trapped ions [495], or dipolar atomic gases [144,775], it is possible to realize complex spin systems with long range interactions that may serve as a model for classical and quantum neural networks [776] (cf. [777]).

Disordered systems offer at least two possible advantages for quantum computing. First, since they have typically a large number of different metastable (free) energy minima, such states might be used to store information distributed over the whole system, similarly to classical neural network (NN) models [778]. The information is thus naturally stored in a redundant way, like in error correcting schemes $[779,780]$. Second, in disordered systems with long range interactions, the stored information is robust: metastable states have quite large basins of attraction in the thermodynamical sense.

The aim of using complex disordered systems is therefore to be able to perform distributed quantum computing in a way that is resistant to noise. In Refs. [781, 782], the following questions in the non-distributed case were addressed: ${ }^{1}$ (i) Can one generate entanglement in such systems that would survive quenched averaging over long times? (ii) Can one realize quantum gates with reasonable fidelity? Both questions were answered affirmatively for both short and long range disordered systems. Here we present as an example, the second question for the Hadamard gate [781].

We assume that the computation is performed in a spin lattice created with cold ions in an optical lattice, of which particles 1 and 2 are part of. At a certain time, particle 1 is in an arbitrary state $\left|\psi_{i n}\right\rangle$, while 2 is in an eigenstate of $\sigma^{z}$ with

\footnotetext{
${ }^{1}$ Since the submission of an earlier version of this review, the distributed case has also been considered $[776,783]$.
} 
eigenvalue +1 e.g. $|+\rangle$. Then we let 1 and 2 evolve according to the EdwardsAnderson Hamiltonian

$$
H_{E-A}=-J \sigma_{1}^{z} \sigma_{2}^{z}
$$

for a suitable duration of time. Here $J$ is a random quenched coupling, that in the case of the Edwards-Anderson model, can be regarded a Gaussian random variable. After a suitable duration of evolution, a measurement is performed on particle 1 (in a suitable basis). For the case when $J$ is a Gaussian variable with mean $=5$ and variance $=1$, the state in particle 2 attains the Hadamard rotated state $\mathbb{H}\left|\psi_{\text {in }}\right\rangle$, with quenched averaged fidelity greater than 0.85 . One can increase such fidelity by increasing the number of spins, and employing measurements assisted by outcomes of previously performed measurements. Note, that the fidelity of the Hadamard rotated state using the classical information obtained only from the measurement of particle 1 , is only $2 / 3$ [784].

\section{Summary}

Summarizing, we have tried to survey the state-of-the-art on the active research field of ultracold atomic gases in optical lattices. On one hand, we have aimed at giving a general outline of new topics and open problems that can be addressed with these systems. Our list, however, is by no means exhaustive. On the other hand, we have briefly reviewed some of the best established methods and techniques to study strongly correlated systems in the framework of ultracold gases. In spite of the fact that we have mostly focused on the connections of ultracold gases with condensed matter topics, we envision and hope that, in the next years, the frontiers of this field will greatly expand.

Finally, we hope we have at least partially convey our enthusiasm for ultracold gases physics. A concise version of our summary is contained in the motto, which has in fact been used by one of us (M.L.) at the end of his conference presentations and seminars. This motto should express a pure joy of curiosity and discovery (even if it was re-discovery) that we and many others have experienced while working on ultracold atomic gases over the last years. This is the most important message: Ultracold atom physics is FA-A-ANTASTIC!!!

\section{Acknowledgements}

This review is to a great extent based on ideas and discussions that we shared with our long lasting friends, close collaborators, and colleagues: Jan Arlt, Alain Aspect, Nuri Barberan, Michael Baranov, Indrani Bose, Immanuel Bloch, Kai Bongs, Sibylle Braungardt, Dagmar Bruß, Ignacio Cirac, Eugene Demler, Kai Eckert, Wolfgang Ertmer, Uli Everts, Henning Fehrmann, Massimo Inguscio, Jarosław Korbicz, Anna Kubasiak, Jonas Larson, Mikko Juhani Leskinen, Alem Mebrahtu, Chiara Menotti, Armand Niederberger, Belen Paredes, Eugene Polzik, Andreas Osterloh, Klaus Osterloh, Carles Rodó, Oriol Romero-Isart, Robert Roth, Kazimierz Rzązewski, Krzysztof Sacha, Laurent Sanchez-Palencia, Luis Santos, Klaus 
Sengstock, Gora Shlyapnikov, Christian Trefzger, Janek Wehr, Jakub Zakrzewski, Łukasz Zawitkowski, and Peter Zoller.

We acknowledge support from the Deutsche Forschungsgemeinschaft (SFB 407, SPP1078 and SPP1116), the Spanish Ministerio de Ciencia y Tecnología grants BFM-2002-02588, FIS2005-04627, FIS2005-01497, FIS2005-01369 and Consolider Ingenio 2010 CSD2006-00019, the Alexander von Humboldt Foundation, the ESF Program QUDEDIS, the EU IP SCALA, and the U.S. Department of Energy.

\section{Appendix A: Effective Hamiltonian to second order}

The Bose Hubbard Hamiltonian or Fermi-Bose Hamiltonian can be split into $H=H_{0}+H_{\text {int }}$, where $H_{0}$ denotes the on-site Hamiltonian obtained in the limit of zero tunneling, and the hopping term is denoted by $H_{\text {int }}$. The Hamiltonian $H_{0}$ can be easily diagonalised and has well defined eigenstates, that are grouped in blocks (or manifolds). Each manifold is well separated from each other. Typically different manifolds are separated by terms of the order of $U$, being $U$ the interaction between two bosons. We denote by $P_{\alpha}$, the projector on each block space, where $\alpha$ is the block index, and the $i$-th state in any block is denoted by $|\alpha, i\rangle$. Note that $P_{\alpha} H_{0} P_{\beta}=0$ holds for $\alpha \neq \beta$. The $H_{\text {int }}$ part of the Hamiltonian will introduce couplings between the bare block $\alpha$ and $\beta$, i.e., $P_{\alpha} H_{\text {int }} P_{\beta}$ can be different from zero for $\alpha \neq \beta$. Following [230], one can construct an effective Hamiltonian, $H_{\text {eff }}$, from $H$ such that it describes the slow, low-energy perturbation-induced tunneling strictly within each manifold of the unperturbed block states and has the same eigenvalues as $H$. Tunneling processes between different manifolds are thus neglected. We demand that the effective hamiltonian $H_{e f f}$ fulfills that:

(i) is by construction hermitian, with the same eigenvalues and the same degeneracies as $H$. To achieve that, one defines $T:=e^{i S}$, with $S$ hermitian, $S=S^{\dagger}$, and chosen such that:

$$
H_{\text {eff }}=T H T^{\dagger} .
$$

(ii) $H_{\text {eff }}$ does not couple states from different manifolds:

$$
P_{\alpha} H_{e f f} P_{\beta}=0, \quad \alpha \neq \beta \quad .
$$

(iii) As the first two conditions still allow for an infinite number of unitary transformations (all $U T$ are still possible, $U$ being any unitary transformation acting only within the manifolds), the following additional condition is imposed:

$$
P_{\alpha} S P_{\alpha}=0 \text { for any } \alpha \text {. }
$$

Expanding the first condition using the Baker-Hausdorff formula, one obtains:

$$
\begin{aligned}
H_{e f f}= & H+[i S, H]+\frac{1}{2 !}[i S,[i S, H]] \\
& +\frac{1}{3 !}[i S,[i S,[i S, H]]]+\ldots .
\end{aligned}
$$


Making a power-series ansatz for in the perturbative parameter $t S$,

$$
S=t S_{1}+t^{2} S_{2}+t^{3} S_{3}+\ldots,
$$

and employing $H=H_{0}+t H_{\text {int }}$ one obtains from (A4) to second order

$$
\begin{aligned}
& H_{\text {eff }}=H_{0}+t \overbrace{\left(\left[i S_{1}, H_{0}\right]+H_{\text {int }}\right)}^{H_{\text {eff }}^{1}} \\
& H_{\text {eff }}^{2} \\
& +t^{2} \overbrace{\left(\left[i S_{2}, H_{0}\right]+\left[i S_{1}, H_{\text {int }}\right]+\frac{1}{2}\left[i S_{1},\left[i S_{1}, H_{0}\right]\right]\right)} .
\end{aligned}
$$

This is a power series for $H_{e f f}$, with its moments denoted by $H_{e f f}^{1}, H_{e f f}^{2}$. to first order, i.e. $H_{e f f}=H_{0}+t H_{e f f}^{1}$ and $S=t S_{1}$. Using the second and third conditions, as well as $P_{\alpha} H_{0} P_{\beta}=0$ and the expression for $H_{\text {eff }}^{1}$ in (A6), one finds:

$$
\begin{gathered}
\left\langle\alpha, i\left|i S_{1}\right| \beta, j\right\rangle\left(E_{\beta j}-E_{\alpha i}\right)+\left\langle\alpha, i\left|H_{i n t}\right| \beta, j\right\rangle=0 \\
\Rightarrow\left\langle\alpha, i\left|i S_{1}\right| \beta, j\right\rangle=\left\{\begin{array}{cc}
\frac{\left\langle\alpha, i\left|H_{i n t}\right| \beta, j\right\rangle}{E_{\alpha i}-E_{\beta j}} & \alpha \neq \beta \\
0 & \alpha=\beta
\end{array} .\right.
\end{gathered}
$$

Thus, the effective Hamiltonian within the $\alpha$-manifold, depends only on $H_{\text {int }}$ and not on $S_{1}$, i.e., $\left\langle\alpha, i\left|H_{e f f}^{1}\right| \alpha, j\right\rangle=\left\langle\alpha, i\left|H_{\text {int }}\right| \alpha, j\right\rangle$. A general result for any $n$ is that $\left\langle\alpha, i\left|H_{e f f}^{n}\right| \alpha, j\right\rangle$ is independent of $S_{n}$. Based on the third condition, and on the observation that $S_{n}$ enters the expression for $H_{e f f}^{n}$ only in the commutator with $H_{0}$, which is diagonal in the manifold index.

Thus, when continuing to second order, the term $\left[i S_{2}, H_{0}\right]$ in the expression for $H_{e f f}^{2}$ can be dropped. Of the two remaining terms defining $H_{\text {eff }}^{2}$ in (A6), the second one can be simplified by observing, that according to (A7) the operator $\left[i S_{1}, H_{0}\right]$ is purely non-diagonal in the manifold index, with values opposite to those of the non-diagonal part of $H_{\text {int }}$. Thus, $\frac{1}{2}\left[i S_{1},\left[i S_{1}, H_{0}\right]\right]=-\frac{1}{2}\left[i S_{1}, H_{i n t}^{n d}\right]$. Now inserting the identity between the operators in the still untreated second term in $H_{\text {eff }}^{2},\left[i S_{1}, H_{\text {int }}\right]$, one sees that due to $S_{1}$ being non-diagonal in $\alpha$, again only the non-diagonal part of $H_{\text {int }}$ can contribute: $\left[i S_{1}, H_{i n t}\right]=\left[i S_{1}, H_{i n t}^{n d}\right]$. Therefore, one has:

$$
H_{\text {eff }}^{2}=\left[i S_{1}, H_{\text {int }}^{n d}\right]+\frac{1}{2}[i S_{1}, \overbrace{\left[i S_{1}, H_{0}\right]}^{-H_{\text {int }}^{\text {nd }}}]=\frac{1}{2}\left[i S_{1}, H_{\text {int }}^{n d}\right] .
$$

Collecting all terms relevant for $\left\langle\alpha, i\left|H_{\text {eff }}\right| \alpha, j\right\rangle$ to second order in $t$, and introducing the notation $Q_{\alpha i}:=\sum_{k, \gamma \neq \alpha} \frac{|\gamma, k\rangle\langle\gamma, k|}{E_{\gamma k}-E_{\alpha i}}$, one finds:

$$
\begin{gathered}
\left\langle\alpha, i\left|H_{e f f}\right| \alpha, j\right\rangle=E_{\alpha i} \delta_{i j}+t\left\langle\alpha, i\left|H_{i n t}\right| \alpha, j\right\rangle \\
-\frac{t^{2}}{2}\left(\left\langle\alpha, i\left|H_{i n t}\left[Q_{\alpha i}+Q_{\alpha j}\right] H_{\text {int }}\right| \alpha, j\right\rangle\right),
\end{gathered}
$$


where the identity operator has been inserted in the final expression for $H_{\text {eff }}^{2}$ in formula (A9), and then evaluated using formula (A8), which naturally leads one to define the operator $Q_{\alpha i}$ as above. Note that this construction can be generalized to arbitrary orders in $t$ in a straightforward manner, as detailed in [230].

\section{Appendix B: Size of the occupation-reduced Hilbert space}

We derive here Eq. (26), i.e., an expression for the size of the Hilbert space in the system subjected to the restriction that at most $K$ bosons can populate a single lattice site. We assume that there are $N$ bosons placed in $M$ lattice sites, therefore $K \leq$ $N$ and $K M \geq N$. By definition $\left.\mathcal{H S}(N, M)\right|_{K}=\sum_{n_{1}=0}^{K} \cdots \sum_{n_{M}=0}^{K} \delta_{n_{1}+\cdots+n_{M}, N}$. Using the following Kronecker delta representation, $\delta_{l, m}=\frac{1}{2 \pi} \int_{-\pi}^{\pi} \mathrm{d} \varphi e^{i(l-m) \varphi}$, one gets

$\left.\mathcal{H} \mathcal{S}(N, M)\right|_{K}=\frac{1}{2 \pi} \int_{-\pi}^{\pi} \mathrm{d} \varphi e^{-i N \varphi}\left(\frac{e^{i(K+1) \varphi}-1}{e^{i \varphi}-1}\right)^{M}=\left.\frac{1}{N !} \frac{d^{N}}{d z^{N}}\left(\frac{z^{K+1}-1}{z-1}\right)^{M}\right|_{z=0}$,

where the right hand side is obtained after integration on a unit circle $z=\exp (i \varphi)$ in the complex plane. Then, using the Leibnitz formula one obtains

$$
\left.\mathcal{H S}(N, M)\right|_{K}=\left.\frac{1}{N !} \sum_{n=0}^{N}\left(\begin{array}{c}
N \\
n
\end{array}\right) \frac{d^{N-n}}{d z^{N-n}}\left(z^{K+1}-1\right)^{M} \frac{d^{n}}{d z^{n}}\left(\frac{1}{z-1}\right)^{M}\right|_{z=0}
$$

which can be further reduced to (26).

\section{References}

[1] William Shakespeare, Hamlet, Prince of Denmark (Cambridge University Press, Cambridge, 2003).

[2] R.J. Glauber, Phys. Rev. 130, 2529 (1963).

[3] R.J. Glauber, Phys. Rev. 131, 2766 (1963).

[4] M.O. Scully and W.E. Lamb, Phys. Rev. 159, 2088 (1967).

[5] H. Haken, Z. Phys. 190, 3278 (1966).

[6] D.F. Walls and G.J. Milburn, Quantum Optics, (Springer, Berlin, 2006).

[7] C.W. Gardiner and P. Zoller, Quantum Noise : A Handbook of Markovian and NonMarkovian Quantum Stochastic Methods with Applications to Quantum Optics, (Springer, Berlin, 2004).

[8] S. Chu, Rev. Mod. Phys. 70, 685 (1998).

[9] C.N. Cohen-Tannoudji, Rev. Mod. Phys. 70, 707 (1998).

[10] W.D. Phillips, Rev. Mod. Phys. 70, 721 (1998).

[11] H.J. Metcalf and P. van der Straten, Laser Cooling and Trapping, (Springer, Berlin, 2001).

[12] P. Meystre, Atom Optics, (American Institute of Physics, Ridge, NY, 2001).

[13] M.H. Anderson, J.R. Ensher, M.R. Matthews, C.E. Wieman, and E.A. Cornell, Science 269, 198 (1995).

[14] K.B. Davis, M.O. Miewes, M.R. Andrews, N.J. van Druten, D.S. Durfee, D.M. Kurn, and W. Ketterle, Phys. Rev. Lett. 75, 3969 (1995).

[15] E.A. Cornell and C.E. Wieman, Rev. Mod. Phys. 74, 875 (2002).

[16] W. Ketterle, Rev. Mod. Phys. 74, 1131 (2002).

[17] L. Pitaevski and S. Stringari, Bose-Eisntein condensation, (Clarendon Press, Oxford, 2003). 
[18] A. Peres, Quantum Theory: Concepts and Methods, (Springer, Berlin, 1993).

19] C.H. Bennett and G. Brassard, IEEE Int. Conf. Comp. Sys. Sig. Proc., 175 (Bangaldore, India, 1984).

[20] A.K. Ekert, Phys. Rev. Lett. 67, 661 (1991)

21] C.H. Bennett and S.J. Wiesner, Phys. Rev. Lett. 69, 2881 (1992).

[22] C.H. Bennett, G. Brassard, C. Crépeau, R. Jozsa, A. Peres, and W.K. Wootters, Phys. Rev. Lett. 70, 1895 (1993).

[23] P.W. Shor, Proc. 35th Ann. Symp. Found. Comp. Sci., (IEEE Press, Los Alamitos, 1994).

[24] J.I. Cirac and P. Zoller, Phys. Rev. Lett. 74, 4091 (1995).

[25] D. Bouwmeester, A.K. Ekert, and A. Zeilinger, The Physics of Quantum Information: Quantum Cryptography, Quantum Teleportation, Quantum Computation, (Springer, Berlin, 2000).

[26] P. Zoller et al., Quantum information processing and communication - Strategic report on current status, visions and goals for research in Europe, Eur. Phys. J. D 36, 203 (2005).

[27] R.P. Feynman, Found. Phys. 16, 507 (1986).

[28] J.I. Cirac and P. Zoller, Physics Today 57, 38 (2004).

[29] D. Jaksch, C. Bruder, J. I. Cirac, C. W. Gardiner, and P. Zoller, Phys. Rev. Lett. 81, 3108 (1998).

[30] M.P.A. Fisher, P.B. Weichman, G. Grinstein, and D.S. Fisher, Phys. Rev. B 40, 546 (1989).

[31] D. Jaksch, H.-J. Briegel, J.I. Cirac, C.W. Gardiner, and P. Zoller, Phys. Rev. Lett. 82, 1975 (1999).

[32] M. Greiner, O. Mandel, T. Esslinger, T. W. Hänsch and I. Bloch, Nature 415, 39 (2002).

[33] I. Bloch, Physics World 17, 25 (2004).

[34] M. Köhl, H. Moritz, T. Stöferle, K. Günter, and T. Esslinger, Phys. Rev. Lett. 94, 080403 (2005).

[35] L. Fallani, J. E. Lye, V. Guarrera, C. Fort, and M. Inguscio, cond-mat/0603655.

[36] S. Ospelkaus, C. Ospelkaus, O. Wille, M. Succo, P. Ernst, K. Sengstock, and K. Bongs, Phys. Rev. Lett. 96, 180403 (2006).

[37] K. Günter, T. Stöferle, H. Moritz, M. Köhl, and T. Esslinger, Phys. Rev. Lett. 96, 180402 (2006).

[38] T. Volz, N. Syassen, D. M. Bauer, E. Hansis, S. Dürr, and G. Rempe, Nature Phys. 2, 692 (2006).

[39] K. Winkler, G. Thalhammer, F. Lang, R. Grimm, J. Hecker-Denschlag, A. J. Daley, A. Kantian, H. P. Büchler, and P. Zoller, Nature 441, 853 (2006).

[40] A.J. Leggett, Rev. Mod. Phys. 73, 307 (2001).

[41] W.V. Liu, F. Wilczek, and P. Zoller, Phys. Rev. A 70, 033603 (2004).

[42] H.D. Politzer, Phys. Rev. A 43, 6444 (1991).

[43] H.-P. Büchler, M. Hermele, S.D. Huber, M.P.A. Fisher, and P. Zoller, Phys. Rev. Lett. 95, 040402 (2005)

[44] M. Aizenman, E.H. Lieb, R. Seiringer, J.P. Solovej, and J. Yngvason, Phys. Rev. A 70, 023612 (2004).

[45] F.H.L. Essler, H. Frahm, F. Göhmann, A. Klümper, and V.E. Korepin, The One-Dimensional Hubbard Model, (Cambridge University Press, Cambridge, 2005).

[46] Th. Giamarchi, Quantum Physics in One Dimension, (Oxford University Press, Oxford, 2004)

[47] U. Schollwöck, Rev. Mod. Phys. 77, 259 (2005).

[48] M.A. Cazalilla, J. Phys. B- Atom. Molec. Phys. 37, S1 (2004).

[49] A. Recati, P.O. Fedichev, W. Zwerger, and P. Zoller, Phys. Rev. Lett. 90, 020401 (2003).

[50] B. Paredes and J.I. Cirac, Phys. Rev. Lett. 90, 150402 (2003).

[51] C. Kollath, U. Schollwöck, and W. Zwerger, Phys. Rev. Lett. 95, 176401 (2005).

[52] B. Paredes, A. Widera, V. Murg, O. Mandel, S. Fölling, J.I. Cirac, G.V. Shlyapnikov, T.W. Hänsch, and I. Bloch, Nature 429, 277 (2004).

[53] H. Moritz, T. Stöferle, M. Köhl, and T. Esslinger, Phys. Rev. Lett. 91, 250402 (2003)

[54] T. Kinoshita, T. Wegner, and D.S. Weiss, Science 305, 1125 (2004).

[55] T. Stöferle, H. Moritz, Ch. Schori, M. Köhl, and T. Esslinger, Phys. Rev. Lett. 93, 240402 (2004).

[56] B. Laburthe Tolra, K.M. O’Hara, J.H. Huckans, W.D. Phillips, S.L. Rolston, and J.V. Porto, Phys. Rev. Lett. 92, 190401 (2004)

[57] A.J. Leggett, S. Chakravarty, A.T. Dorsey, M.P.A. Fisher, and W. Zwerger, Rev. Mod. Phys. 59, 1 (1987).

[58] A. Recati, P.O. Fedichev, W. Zwerger, J. von Delft, and P. Zoller, Phys. Rev. Lett. 94, 040404 
(2005).

[59] Z. Hadzibabic, P. Krüger, M. Cheneau, B. Battelier, and J.B. Dalibard, Nature 441, 1118 (2006).

[60] A. Auerbach, Interacting Electrons and Quantum magnetism, (Springer, New York, 1994).

61] D. Jaksch and P. Zoller, Ann. Phys. (N.Y.) 315, 52 (2005).

[62] J.J. García-Ripoll, M.A. Martin-Delgado, and J.I. Cirac, Phys. Rev. Lett. 93, 250405 (2004).

[63] L. Santos, M.A. Baranov, J.I. Cirac, H.-U. Everts, H. Fehrmann, and M. Lewenstein, Phys. Rev. Lett. 93, 030601 (2004).

[64] L.-M. Duan, E. Demler, and M.D. Lukin, Phys. Rev. Lett. 91, 090402 (2003).

[65] U. Dorner, P. Fedichev, D. Jaksch, M. Lewenstein, and P. Zoller, Phys. Rev. Lett. 91, 073601 (2003).

[66] P. W. Anderson, Phys. Rev. 109, 1492 (1958)

[67] N.F. Mott, Rev. Mod. Phys. 40, 677 (1968).

[68] B. Damski, J. Zakrzewski, L. Santos, P. Zoller, and M. Lewenstein, Phys. Rev. Lett. 91, $080403(2003)$.

[69] R. Roth and K. Burnett, J. Opt. B: Quantum Semiclass. Opt. 5, S50 (2003).

[70] R. Roth and K. Burnett, Phys. Rev. A 68, 023604 (2003).

[71] J. E. Lye, L. Fallani, M. Modugno, D. Weirsma, C. Fort, and M. Inguscio, Phys. Rev. Lett. 95, 070401 (2005)

[72] C. Fort, L. Fallani, V. Guarrera, J. Lye, M. Modugno, D. S. Wiersma, and M. Inguscio, Phys. Rev. Lett. 95, 170410 (2005).

[73] T. Schulte, S. Drenkelforth, J. Kruse, W. Ertmer, J. Arlt, K. Sacha, J. Zakrzewski, and M. Lewenstein, Phys. Rev. Lett. 95, 170411 (2005).

[74] D. Clément, A. F. Varón, M. Hugbard, J. A. Retter, P. Bouyer, L. Sanchez-Palencia, D. M. Gangardt, G. V. Shlyapnikov, and A. Aspect, Phys. Rev. Lett. 95, 170409 (2005).

[75] N. Bilas and N. Pavloff, Phys. Rev. Lett. 95, 130403 (2005).

[76] T. Paul, P. Leboeuf, N. Pavloff, K. Richter, and P. Schlagheck, cond-mat/0509446.

[77] R.C. Kuhn, C. Miniatura, D. Delande, O. Sigwarth, and C.A. Müller, Phys. Rev. Lett. 95, $250403(2005)$.

[78] N. Bilas and N. Pavloff, cond-mat/0602622.

[79] T. Schulte, S. Drenkelforth, J. Kruse, R. Tiemeyer, K. Sacha, J. Zakrzewski, M. Lewenstein, W. Ertmer, and J.J. Arlt, New J. Phys. 8, 230 (2006).

[80] P. Lugan, D. Clément, P. Bouyer, A. Aspect, M. Lewenstein, and L. Sanchez-Palencia, condmat/0610389.

[81] S. F. Edwards and P. W. Anderson, J. Phys. F 5, 965 (1975).

[82] D. Sherrington and S. Kirkpatrick, Phys. Rev. Lett. 35, 1792 (1975).

[83] M. Mézard, G. Parisi, and M.A. Virasoro, Spin Glass Theory and Beyond (World Scientific, Singapore, 1987).

[84] D. S. Fisher and D. A. Huse, Phys. Rev. Lett. 56, 1601 (1986).

[85] A. J. Bray and M. A. Moore, Phys. Rev. Lett. 58, 57 (1987).

[86] A. Sanpera, A. Kantian, L. Sanchez-Palencia, J. Zakrzewski, and M. Lewenstein, Phys. Rev. Lett. 93, 040401 (2004).

[87] V. Ahufinger, L. Sanchez-Palencia, A. Kantian, A. Sanpera, and M. Lewenstein, Phys. Rev. A 72,063616 (2005).

[88] J. Wehr, A. Niederberger, L. Sanchez-Palencia, and M. Lewenstein, cond-mat/0604063.

[89] T. Claeson and P. Delsing (Eds.), Condensation and Coherence in Condensed Matter, Proc. of the Nobel Jubilee Symposium, Göteborg 2001, (Physica Scripta/The Royal Academy of Sciences/World Scientific, Singapore, 2003).

[90] W. Hofstetter, J.I. Cirac, P. Zoller, E. Demler, and M.D. Lukin, Phys. Rev. Lett. 89, 220407 (2005).

[91] A.O. Koetsier, D.B.M. Dickerscheid, H.T.C. Stoof, Phys. Rev. A 74, 033621 (2006).

[92] T. Stöferle, H. Moritz, K. Günter, M. Köhl, and T. Esslinger, Phys. Rev. Lett. 96, 030401 (2006).

[93] S. Inouye, M.R. Andrews, J. Stenger, H.-J. Miesner, D.M. Stamper-Kurn, and W. Ketterle, Nature 392, 151 (1998).

[94] S.L. Cornish, N.R. Claussen, J.L. Roberts, E.A. Cornell, and C.E. Wieman, Phys. Rev. Lett. 85, 1795 (2000).

[95] E. Timmermans, P. Tommasini, M. Hussein, and A. Kerman, Phys. Rep. 315, 199 (1999).

[96] C.A. Regal, M. Greiner, and D.S. Jin, Phys. Rev. Lett. 92, 040403 (2004).

[97] M. Bartenstein, A. Altmeyer, S. Riedl, S. Jochim, C. Chin, J.H. Denschlag, and R. Grimm, 
Phys. Rev. Lett. 92, 203201 (2004).

[98] C. Chin, M. Bartenstein, A. Altmeyer, S. Riedl, S. Jochim, J.H. Denschlag, and R. Grimm, Science 305, 1128 (2004).

[99] M.W. Zwierlein, C.A. Stan, C.H. Schunck, S.M.F. Raupach, A.J. Kerman, and W. Ketterle, Phys. Rev. Lett. 92, 120403 (2004).

[100] J. Kinast, S.L. Hemmer, M.E. Gehm, A. Turlapov, and J.E. Thomas, Phys. Rev. Lett. 92, $150402(2004)$.

[101] J. Kinast, A. Turlapov, J.E. Thomas, Q.J. Chen, J. Stajic, and K. Levin, Science 307, 1296 (2005).

[102] T. Bourdel, L. Khaykovich, J. Cubizolles, J. Zhang, F. Chevy, M. Teichmann, L. Tarruell, S.J.J.M.F. Kokkelmans, and C. Salomon, Phys. Rev. Lett. 93, 050401 (2004).

[103] G.B. Partridge, K.E. Strecker, R.I. Kamar, M.W. Jack, and R.G. Hulet, Phys. Rev. Lett. 95, 020404 (2005).

[104] M.W. Zwierlein, J.R. Abo-Shaeer, A. Schirotzek, C.H. Schunck, and W. Ketterle, Nature 435, 1047 (2005).

[105] M.W. Zwierlein, A. Schirotzek, C.H. Schunck, and W. Ketterle, Science 311, 492 (2006).

[106] G.B. Partridge, W.H. Li, R.I. Kamar, Y.A. Liao, and R.G. Hulet, Science 311, 503 (2006).

[107] G. Misguich and C. Lhuillier, Two-dimensional quantum anti-ferromagnets (condmat/0310405), in Frustrated spin systems, edited by H.T. Diep (World Scientific, Singapore, 2004).

[108] C. Lhuillier, cond-mat/0502464.

[109] F. Alet, A.M. Walczak, and M.P.A. Fisher, Physica A 369, 122 (2006) (cond-mat/0511516).

[110] B. Damski, H.-U. Everts, A. Honecker, H. Fehrmann, L. Santos, and M. Lewenstein, Phys. Rev. Lett. 95, 060403 (2005).

[111] B. Damski, H. Fehrmann, H.-U. Everts, M. Baranov, L. Santos, and M. Lewenstein, Phys. Rev. A 72, 053612 (2005).

[112] A. Honecker, D.C. Cabra, H.-U. Everts, P. Pujol, and F. Stauffer, cond-mat/0609312.

[113] A. Kitaev, Ann. Phys. (N.Y.) 321, 2 (2006).

[114] B. Douçot, M.V. Feigel'man, L.B. Ioffe, A.S. Ioselevich, Phys. Rev. B 71, 024505 (2005).

[115] A. Micheli, G.K. Brennen, and P. Zoller, Nature Physics 2, 341 (2006).

[116] M. Lewenstein, Nature Physics 2, 289 (2006).

[117] D. Stamper-Kurn and W. Ketterle, cond-mat/0005001, Proceedings of Les Houches 1999 Summer School, Session LXXII.

[118] E. Demler and F. Zhou, Phys. Rev. Lett. 88, 163001 (2002).

[119] A. Imambekov, M. Lukin, and E. Demler, Phys. Rev. A 68, 063602 (2003).

[120] S.K. Yip, Phys. Rev. Lett. 90, 250402 (2003).

[121] S.K. Yip, J. Phys. C - Cond. Mat. 15, 4583 (2003).

122 Ł. Zawitkowski, K. Eckert, M. Laskinen, A. Sanpera, and M. Lewenstein, cond-mat/0603273.

[123] R.B. Laughlin, Phys. Rev. Lett. 50, 1395 (1983).

[124] L. Jacak, P. Sitko, K. Wieczorek, and A. Wójs, Quantum Hall Systems : Braid Groups, Composite Fermions, and Fractional Charge, (Oxford University Press, Oxford, 2003).

[125] N.K. Wilkin, and J.M.F. Gunn, Phys. Rev. Lett. 84, 6 (2000).

[126] N.R. Cooper, N.K. Wilkin, and J.M.F. Gunn, Phys. Rev. Lett. 87, 120405 (2001).

[127] B. Paredes, P. Fedichev, J.I. Cirac, and P. Zoller, Phys. Rev. Lett. 87, 010402 (2001).

$128]$ M. Popp, B. Paredes, and J.I. Cirac, Phys. Rev. A 70, 053612 (2004).

[129] N. Barberan, M. Lewenstein, K. Osterloh, and D. Dagnino, Phys. Rev. A 73, 063623 (2006); D. Dagnino, N. Barbern, M. Lewenstein, K. Osterloh, A. Riera, cond-mat/0610512.

[130] D. Jaksch and P. Zoller, New J. Phys. 5, Art. No. 56 (2003).

[131] E.J. Mueller, Phys. Rev. A 70, 041603 (2004).

[132] A.S. Sorensen, E. Demler, and M.D. Lukin, Phys. Rev. Lett. 94, 086803 (2005).

[133] R.N. Palmer and D. Jaksch, Phys. Rev. Lett. 96, 180407 (2006).

[134] I. Montvay and G. Münster, Quantum Fields of a Lattice (Cambridge University Press, Cambridge, 1997).

[135] K. Osterloh, M. Baig, L. Santos, P. Zoller, and M. Lewenstein, Phys. Rev. Lett. 95, 010403 (2005).

[136] J. Ruseckas, G. Juzeliunas, P. Öhberg, and M. Fleischhauer, Phys. Rev. Lett. 95, 010404 (2005)

[137] J.K. Pachos and E. Rico, Phys. Rev. A 70, 053620 (2004).

[138] S. Tewari, V.W. Scarola, T. Senthil, and S. Das Sarma, cond-mat/0605154.

[139] D. Jaksch, V. Venturi, J.I. Cirac, C.J. Williams, and P. Zoller, Phys. Rev. Lett. 89, 040402 
(2002).

[140] B. Damski, L. Santos, E. Tiemann, M. Lewenstein, S. Kotochigova, P. Julienne, and P. Zoller, Phys. Rev. Lett. 90, 110401 (2003)

[141] T. Rom, T. Best, O. Mandel, A. Widera, M. Greiner, T.W. Hänsch, and I. Bloch, Phys. Rev. Lett. 93, 073002 (2004).

[142] T. Kraemer, M. Mark, P. Waldburger, J.G. Danzl, C. Chin , B. Engeser, A.D. Lange, K. Pilch, A. Jaakkola, H.C. Nagerl, and R. Grimm, Nature 440, 315 (2006).

[143] M. Stoll, and T. Köhler, Phys. Rev. A 72, 022714 (2005).

[144] M. Baranov, Ł. Dobrek, K. Góral, L. Santos, and M. Lewenstein, Physica Scripta 102, 74 (2002).

[145] A. Griesmaier, J. Werner, S. Hensler, J. Stuhler, and T. Pfau, Phys. Rev. Lett. 94, 160401 (2005).

[146] Special issue on Ultracold Polar Molecules: Formation and Collisions, Eur. Phys. J. D, 31 (2004).

[147] K. Góral, L. Santos, and M. Lewenstein, Phys. Rev. Lett. 88, 170406 (2002).

[148] C. Trefzger, C. Menotti, and M. Lewenstein, in preparation.

[149] N.R. Cooper, E.H. Rezayi, and S.H. Simon, Phys. Rev. Lett. 95, 200402 (2005).

[150] M.A. Baranov, K. Osterloh, and M. Lewenstein, Phys. Rev. Lett. 94, 070404 (2005).

[151] M.A. Baranov, H. Fehrmann, and M. Lewenstein, in preparation.

[152] R. Grimm, M. Weidemüller, and Y.B. Ovchinnikov, Adv. Atom. Molec. Opt. Phys. 42, 95 (2000).

[153] I. Bloch and M. Greiner, Adv. At. Molec. Opt. Phys. 52, 1 (2005).

[154] S. Sachdev, Quantum Phase Transitions, (Cambridge University Press, Cambridge, 1999).

[155] N.W. Ashcroft and N.D. Mermin, Solid State Physics (Holt, Rinehart, and Winston, Philadelphia, 1976).

[156] O.E. Alon, A.I. Streltsov, and L.S. Cederbaum, Phys. Rev. Lett. 95, 030405 (2005).

[157] K. Huang, Statistical Mechanics, (Wiley, New York, 1987).

[158] Z. Idziaszek and T. Callarco, Phys. Rev. Lett. 96, 013201 (2006)

[159] A. De Martino, M. Thorwart, R. Egger and R. Graham, Phys. Rev. Lett. 94, 060402 (2005).

[160] J. Mompart, K. Eckert, W. Ertmer, G. Birkl, and M. Lewenstein, Phys. Rev. Lett. 90 , 147901 (2003).

[161] F. Werner, O. Parcollet, A. Georges, and S.R. Hassan, Phys. Rev. Lett. 95, 056401 (2005).

[162] P. Rabl, A.J. Daley, P.O. Fedichev, J.I. Cirac, and P. Zoller, Phys. Rev. Lett. 91, 110403 (2005).

[163] A. Griessner, A.J. Daley, D. Jaksch, and P. Zoller, Phys. Rev. A 72, 032332 (2005).

[164] M. Popp, J. J. García-Ripoll, K. G. H. Vollbrecht, and J. I. Cirac, Phys. Rev. A 74, 013622 (2006).

[165] M. Popp, J.-J. García-Ripoll, K.G.H. Vollbrecht, and J.I. Cirac, New J. Phys. 8, 164 (2006).

[166] A. B. Kuklov and B. V. Svistunov, Phys. Rev. Lett. 90, 100401 (2003).

[167] M. Lewenstein, L. Santos, M. A. Baranov and H. Fehrmann, Phys. Rev. Lett. 92, 050401 (2004).

[168] R. Barnett, A. Turner, and E. Demler, Phys. Rev. Lett. 97, 180412 (2006).

[169] F. Zhou and G.W. Semenoff, Phys. Rev. Lett. 97, 180411 (2006).

[170] L. Amico, A. Osterloh, and F. Cataliotti, Phys. Rev. Lett. 95, 063201 (2005).

[171] C. Maschler and H. Ritsch, Phys. Rev. Lett. 95, 260401 (2005).

[172] D. Nagy, J.K. Asboth, P. Domokos, and H. Ritsch, Europhys. Lett. 74, 254 (2006).

[173] J. Larson, B. Damski, G. Morigi, and M. Lewenstein, cond-mat/0608335.

[174] M. Lewenstein, A. Kubasiak, J. Larson, C. Menotti, G. Morigi, K. Osterloh, and A. Sanpera, submitted to the Proceedings of ICAP-2006 (cond-mat/0609587).

[175] P.O. Fedichev, Y. Kagan, G.V. Shlyapnikov, and J.T.M. Walraven, Phys. Rev. Lett. 77, 2913 (1996).

[176] G. Thalhammer, M. Theis, K. Winkler, R. Grimm, and J.H. Denschlag, Phys. Rev. A 71 , 033403 (2005).

[177] M. Theis, G. Thalhammer, K. Winkler, M. Hellwig, G. Ruff, R. Grimm, and J.H. Denschlag, Phys. Rev. Lett. 93, 123001 (2005).

[178] R. Barnett, D. Petrov, M. Lukin, and E. Demler, Phys. Rev. Lett. 96, 190401 (2006).

[179] J. Stuhler, A. Griesmaier, T. Koch, M. Fattori, T. Pfau, S. Giovanazzi, P. Pedri, and L. Santos, Phys. Rev. Lett. 95, 150406 (2005).

[180] L. Della Pietra, S. Aigner, Ch. vom Hagen, S. Groth, I. Bar-Joseph, H.J. Lezec, and J. Schmiedmayer, cond-mat/0604619. 
[181] S. Hofferberth, I. Lesanovsky, B. Fischer, J. Verdu, and J. Schmiedmayer, Nature Phys. 2, $710(2006)$

[182] R. Gati, B. Hemmerling, J. Folling, M. Albiez, and M.K. Oberthaler, Phys. Rev. Lett. 96, 130404 (2006)

[183] H. Fehrmann, M. A. Baranov, B. Damski, M. Lewenstein, and L. Santos, Opt. Comm. 243 , 23 (2004).

[184] R. Roth and K. Burnett, Phys. Rev. A 67, 031602(R) (2003).

[185] E.H. Lieb, R. Seiringer, and J. Yngvason, Phys. Rev. B 66, 134529 (2002).

[186] R.V. Pai, R. Pandit, H.R. Krishnamurthy, and S. Ramasesha, Phys. Rev. Lett. 76, 2937 (1996).

[187] W. Krauth, Phys. Rev. B 44, 9772 (1991).

[188] J.M. Kosterlitz and D.J. Thouless, J. Phys. C 6, 1181 (1973).

[189] M. Campostrini, M. Hasenbusch, A. Pelissetto, P. Rossi, and E. Vicari, Phys. Rev. B 63, 214503 (2001)

[190] B. Damski and J. Zakrzewski, Phys. Rev. A 74, 043609 (2006).

[191] T.D. Kühner, S.R. White, and H. Monien, Phys. Rev. B 61, 12474 (2000).

[192] S. Wessel, F. Alet, M. Troyer, and G.G. Batrouni, Phys. Rev. A 70, 053615 (2004).

[193] J.K. Freericks and H. Monien, Phys. Rev. B 53, 2691 (1996).

[194] G.G. Batrouni, R.T. Scalettar, and G.T. Zimanyi, Phys. Rev. Lett. 65, 1765 (1990).

[195] G.G. Batrouni and R.T. Scalettar, Phys. Rev. B 46, 9051 (1992).

[196] W.V. Liu and C. Wu, Phys. Rev. A 74, 013607 (2006).

[197] C. Wu, W.V. Liu, J. Moore, and S. Das Sarma, Phys. Rev. Lett. 97, 190406 (2006).

[198] P.A. Lee, N. Nagaosa, and X.G. Wen, Rev. Mod. Phys. 78, 17 (2006).

[199] A. Klein and D. Jaksch, Phys. Rev. A 73, 053613 (2006).

[200] J. Kinnunen, L.M. Jensen, and P. Torma, Phys. Rev. Lett. 96, 110403 (2006).

[201] T. Koponen, J.-P. Martikainen, J. Kinnunen, and P. Torma, Phys. Rev. A 73, 033620 (2006).

[202] T. Koponen, J. Kinnunen, J.-P. Martikainen, L.M. Jensen, and P. Torma, New J. Phys. 8, 179 (2006).

[203] C. Ates and K. Ziegler, Phys. Rev. A 71, 063610 (2005).

[204] K. Ziegler, Las. Phys. 16, 699 (2006).

[205] K. Ziegler, cond-mat/0611010.

[206] W. Hofstetter, Phil. Mag. 86, 1891 (2006).

[207] P. Lecheminant, E. Boulat, and P. Azaria, Phys. Rev. Lett. 95, 240402 (2005).

[208] D. Controzzi and A.M. Tsvelik, Phys. Rev. Lett. 95, 097205 (2006).

[209] This phenomenon, related to the appearance of counterflow superfluidity in Ref. [166], may occur also in the absence of the optical lattice; see M.Yu. Kagan, I.V. Brodsky, D.V. Efremov, and A.V. Klaptsov, Physics of Metals and Metallography 99, 225 (2005) (cond-mat/0209481); Phys. Rev. A 70, 023607 (2004)

[210] H. Fehrmann, M. A. Baranov, M. Lewenstein, and L. Santos, Opt. Express 12, 55 (2004).

[211] K. Mølmer, Phys. Rev. Lett. 80, 1804 (1998).

[212] M.J. Bijlsma, B.A. Heringa, and H.T.C. Stoof, Phys. Rev. A 61, 053601 (2000); H. Pu, W. Zhang, M. Wilkens, and P. Meystre, Phys. Rev. Lett. 88, 070408 (2002).

[213] R. Roth, Phys. Rev. A 66, 013614 (2002).

[214] P. Capuzzi and E. S. Hernández, Phys. Rev. A 64, 043607 (2001); X.-J. Liu and H. Hu, ibid. 67, 023613 (2003).

[215] A. Albus et al., Phys. Rev. A 65, 053607 (2002); L. Viverit, and S. Giorgini, ibid. 66, 063604 (2002); H. Heiselberg, C. J. Pethick, H. Smith and L. Viverit, Phys. Rev. Lett. 85, 2418 (2000).

[216] G. Modugno, G. Roati, F. Riboli, F. Ferlaino, R.J. Brecha, and M. Inguscio, Science 297, 2240 (2002); M. Modugno, F. Ferlaino, F. Riboli, G. Roati, G. Modugno, and M. Inguscio, Phys. Rev. A 68, 043626 (2003).

[217] K.K. Das, Phys. Rev. Lett. 90, 170403 (2003); M.A. Cazalilla and A.F. Ho, ibid. 91, 150403 (2003).

[218] G. Modugno, F. Ferlaino, R. Heidemann, G. Roati, and M. Inguscio, Phys. Rev. A 68, 011601(R) (2003); A. Albus, F. Illuminati, and J. Eisert, Phys. Rev. A 68, 023606 (2003); H.P. Büchler and G. Blatter, Phys. Rev. Lett. 91, 130404 (2003); F. Illuminati and A. Albus, Phys. Rev. Lett. 93, 090406 (2004); A. B. Kuklov and B. V. Svistunov, Phys. Rev. Lett. 90, 100401 (2003).

[219] A. Mehring, U. Schollwöck, and M. Fleischhauer, Ver. der Deutsch. Phys. Ges., 69 Jahrestagung Berlin 2005, Kurzfassungen der Vorträge, Q32.6. 
[220] M. Cramer, J. Eisert, and F. Illuminati, Phys. Rev. Lett. 93, 190405 (2004).

[221] A. Imambekov and E. Demler, Phys. Rev. A 73, 021602(R) (2006); Ann. Phys. 321, 2390 (2006) (cond-mat/0510801).

[222] H. Frahm and G. Palacios, Phys. Rev. A 72, 061604(R) (2005).

[223] L.D. Carr and M.J. Holland, Phys. Rev. A 72, 031604 (2005).

[224] F. Zhou, Phys. Rev. B 72, 220501(R) (2005).

[225] R.B. Diener and T.-L. Ho, Phys. Rev. Lett. 96, 010402 (2006).

[226] K. Günter, T. Stöferle, H. Moritz, M. Köhl, and T. Esslinger, Phys. Rev. Lett. 96, 180402 (2006).

[227] S. Ospelkaus, C. Ospelkaus, O. Wille, M. Succo, P. Ernst, K. Sengstock, and K. Bongs, Phys. Rev. Lett. 96, 180403 (2006).

[228] L. Pollet, C. Kollath, U. Schollwoeck, and M. Troyer, cond-mat/0609604.

[229] C. Kittel, Introduction to Solid State Physics, (J. Wiley \& Sons, new York, 2004).

[230] C. Cohen-Tannoudji, J. Dupont-Roc, G. Grynberg, Atom-photon Interactions: Basic Processes and Applications (Wiley, New York, 1992).

[231] S.R. White, Phys. Rev. Lett. 69, 2863 (1992).

[232] A.O. Gogolin, A.A. Nersesyan, and A.M. Tsvelik, Bosonization and Strongly Correlated Systems, (Cambridge University Press, Cambridge, 2004).

[233] V.E. Korepin and F.H.L. Essler, Exactly Solvable Models of Strongly Correlated Electrons, (World Scientific, Singapore, 1994).

[234] V.E. Korepin, N.M. Bogoliubov, and A.G. Izergin, Quantum Inverse Scattering Method and Correlation Functions, (Cambridge University Press, Cambridge, 1997).

[235] A.M. Tsvelik, Quantum Field Theory in Condensed Matter Physics, (Cambridge University Press, Cambridge, 2003).

[236] D. van Oosten, P. van der Straten, and H.T.C. Stoof, Phys. Rev. A 63, 053601 (2001).

[237] F.M. Cucchietti, B. Damski, J. Dziarmaga, and W.H. Zurek, cond-mat/0601650.

[238] M.P. Gelfand, R.R.P. Singh, and D.A. Huse, J. Stat. Phys. 59, 1093 (1990)

[239] M.P. Gelfand, Solid State Commun. 98, 11 (1996).

[240] N. Elstner and H. Monien, Phys. Rev. B 59, 12184 (1999); cond-mat/9905367.

[241] J.K. Freericks and H. Monien, Europhys. Lett. 26, 545 (1994).

[242] T. D. Kühner and H. Monien, Phys. Rev. B 58, R14741 (1998).

[243] T.P. Meyrath, F. Schreck, J.L. Hanssen, C.-S. Chuu, and M.G. Raizen, Phys. Rev. A 71, 041604(R) (2005).

[244] W. Krauth, M. Caffarel, and J.-P. Bouchaud, Phys. Rev. B 45, 3137 (1992).

[245] D.S. Rokhsar and B.G. Kotliar, Phys. Rev. B 44, 10328 (1991).

[246] W. Zwerger, J. Opt. B 5, S9 (2003).

[247] W.H. Press, B.P. Flannery, S.A. Teukolsky, and W.T. Vetterling, Numerical Recipes (Cambridge University Press 1987); http://www.nr.com/.

[248] J. Zakrzewski, Phys. Rev. A 71, 043601 (2005).

[249] L. Amico and V. Penna, Phys. Rev. Lett 80, 2189 (1998); Phys. Rev. B 62, 1224 (2000).

[250] V.W. Scarola, E. Demler, S. Das Sarma, Phys. Rev. A 73, 051601(R) (2006).

[251] C. Schroll, F. Marquardt, and C. Bruder, Phys. Rev. A 70, 053609 (2004).

[252] D.B.M. Dickerscheid, D. van Oosten, P.J.H. Denteneer, and H.T.C. Stoof, Phys. Rev. A 68, $043623(2003)$

[253] S. Konabe, T. Nikuni, and M. Nakamura, Phys. Rev. A 73, 033621 (2006).

254] Y. Ohashi, M. Kitaura and H. Matsumoto, Phys. Rev. A 73, 033617 (2006).

[255] K. Sengupta and N. Dupuis, Phys. Rev. A 71, 033629 (2005).

[256] K. Sheshadri, H.R. Krishnamurthy, R. Pandit and T.V. Ramakrishnan, Europhys. Lett. 22, 257 (1993).

[257] C. Menotti and N. Trivedi, in preparation.

[258] http://www.caam.rice.edu/software/ARPACK/.

[259] R.M. Noack and S.R. Manmana, AIP Conf. Proc. 789, 93 (2005).

[260] W. Krauth, N. Trivedi, and D. Ceperley, Phys. Rev. Lett. 67, 2307 (1991).

[261] W. Krauth and N. Trivedi, Europhys. Lett. 14, 627 (1991).

[262] R.T. Scalettar, G.G. Batrouni, and G.T. Zimanyi, Phys. Rev. Lett. 66, 3144 (1991)

[263] V.A. Kashurnikov, N.V. Prokofév, and B.V. Svistunov, Phys. Rev. A 66, 031601(R) (2002).

[264] N.V. Prokofév, B.V. Svistunov, and I.S. Tupitsyn, Phys. Lett. A 238, 253 (1998); JETP 87, 310 (1998).

[265] F. Alet, S. Wessel, and M. Troyer, Phys. Rev. E 71, 036706 (2005).

[266] F. Alet et al., J. Phys. Soc. Jpn. Suppl. 74, 30 (2005); http://alps.comp-phys.org/. 
[267] G.G. Batrouni, V. Rousseau, R.T. Scalettar, M. Rigol, A. Muramatsu, P.J.H. Denteneer, and M. Troyer, Phys. Rev. Lett. 89, 117203 (2002).

[268] G.G. Batrouni, F.F. Assaad, R.T. Scalettar, and P.J.H. Denteneer, Phys. Rev. A 72, $031601(\mathrm{R})(2005)$.

[269] P. Sengupta, M. Rigol, G.G. Batrouni, P.J.H. Denteneer, and R.T. Scalettar, Phys. Rev. Lett. 95, 220402 (2005).

[270] G.K. Campbell, J. Mun, M. Boyd, P. Medley, A.E. Leanhardt, L. Marcassa, D.E. Pritchard, and W. Ketterle, cond-mat/0606642.

[271] S. Foelling, A. Widera, T. Mueller, F. Gerbier, and I. Bloch, cond-mat/0606592.

[272] O. Gygi, H.G. Katzgraber, M. Troyer, S. Wessel, and G.G. Batrouni, Phys. Rev. A 73, 063606 (2006)

[273] F. Gerbier, A. Widera, S. Fölling, O. Mandel, T. Gericke, and I. Bloch, Phys. Rev. Lett. 95, 050404 (2005); Phys. Rev. A 72, 053606 (2005).

[274] E. Wigner, Phys. Rev. 40, 749 (1932).

[275] E. P. Wigner, Gruppentheorie und ihre Anwendung auf die Quantenmechanik der Atomspektren (F. Vieweg und Sohn, Braunschweig, 1931); English translation: Group Theory and its Application to the Quantum Mechanics of Atomic Spectra, expanded and improved ed. (Academic Press, New York, 1959).

[276] J. E. Moyal, Proc. Camb. Phil. Soc. 45, 99 (1949)

[277] E.C.G. Sudarshan, Phys. Rev. Lett. 10, 277 (1963).

[278] M. J. Steel, M. K. Olsen, L. I. Plimak, P. D. Drummond, S. M. Tan, M. J. Collett, D. F. Walls, and R. Graham, Phys. Rev. A 58, 4824 (1998); P.D. Drummond, P. Deuar, and K.V. Kheruntsyan, Phys. Rev. Lett. 92, 040405 (2004); P. D. Drummond and P. Deuar, J.Opt.B Quant.Semiclass.Opt. 5, 281 (2003); P. D. Drummond, P. Deuar, J. F. Corney, and K. V. Kheruntsyan, cond-mat/0309537, published in Proceedings of the 16th International Conference on Laser Spectroscopy (ICOLS), Palm Cove, Australia (2003); P. Deuar and P. D. Drummond, J. Phys. A: Math. Gen. 39, 1163 (2006); ibid. J. Phys. A: Math. Gen. 39 2723 (2006); for a review see P. D. Drummond and J. F. Corney, Comp. Phys. Comm. 169, $412(2005)$.

[279] J. F. Corney and P. D. Drummond, Phys. Rev. Lett. 93, 260401 (2004) J. F. Corney and P. D. Drummond, Phys. Rev. B 73, 125112 (2006); P. D. Drummond, J. F. Corney, X.-J. Liu, and $\mathrm{H} . \mathrm{Hu}$, in print in J. Mod. Opt. (2006).

[280] A. Sinatra, Y. Castin, and C. Lobo, J. Mod. Opt. 47, 2629 (2000); A. Sinatra, C. Lobo, and Y. Castin, Phys. Rev. Lett. 87, 210404 (2001); I. Carusotto and Y. Castin, J. Phys. B 34, 4589 (2001); A. Sinatra, C. Lobo, and Y. Castin, J. Phys. B 35, 3599 (2002); I. Carusotto and Y. Castin, Phys. Rev. Lett. 90, 030401 (2003); I. Carusotto and Y. Castin, Laser Phys. 13, 509 (2003).

[281] I. Carusotto and Y. Castin, New J. Phys. 5, 91 (2003).

[282] Y. Castin and I. Carusotto, Opt. Comm. 243, 81 (2004).

[283] A. Montina and Y. Castin, Phys. Rev. A 73013618 (2006).

[284] S. Dettmer, D. Hellweg, P. Ryytty, J.J. Arlt, W. Ertmer, K. Sengstock, D.S. Petrov, G.V. Shlyapnikov, H. Kreutzmann, L. Santos, and M. Lewenstein, Phys. Rev. Lett. 87, 160406 (2001).

[285] D. Hellweg, S. Dettmer, P. Ryytty, J. J. Arlt, W. Ertmer, K. Sengstock, D. S. Petrov, G. V. Shlyapnikov, H. Kreutzmann, L. Santos, and M. Lewenstein, Appl. Phys. B 73, 781 (2001).

[286] H. Kreutzmann, A. Sanpera, L. Santos, M. Lewenstein, D. Hellweg, L. Cacciapuoti, M. Kottke, T. Schulte K. Sengstock, J. J. Arlt, and W. Ertmer, cond-mat/0210348.

[287] A. Mebrahtu, A. Sanpera, and M. Lewenstein, Phys. Rev. A 73, 033601 (2006).

[288] J. Mur-Petit, M. Guilleumas, A. Polls, A. Sanpera, M. Lewenstein, K. Bongs, and K. Sengstock, Phys. Rev. A 73, 013629 (2006).

[289] M. J. Davis, S. A. Morgan, and K. Burnett, Phys. Rev. Lett. 87, 160402 (2001); M. J. Davis, R. J. Ballagh, and K. Burnett, J. Phys. B 34, 4487 (2001); M.J. Davis, S.A. Morgan, and K. Burnett, Phys. Rev. A 66, 053618 (2002).

[290] K. Góral, M. Gajda, and K. Rzążewski, Opt. Express 8, 92 (2001); K. Góral, M. Gajda, and K. Rzążewski, Phys. Rev. A 66, 051602 (2002); H. Schmidt, K. Góral, F. Floegel, M. Gajda, and K. Rzążewski, J. Opt. B 5, S96 (2003); M. Brewczyk, P. Borowski, M. Gajda, and K. Rzążewski, J. Phys. B: At. Mol. Opt. Phys. 37, 2725 (2004); Ł. Zawitkowski, M. Brewczyk, M. Gajda, and K. Rzążewski, cond-mat/0406630; K. Gawryluk, M. Brewczyk, and K. Rzażewski, J. Phys. B: At. Mol. Opt. Phys. 39,L225 (2006).

[291] F. Verstraete and J.I. Cirac, J. Stat. Mech. P09012 (2005). 
[292] G.D. Mahan, Many-Particle Physics, (Plenum Press, New York, 1993).

[293] M.A. Cazalilla, A.F. Ho, and T. Giamarchi, New J. Phys. 8, 158 (2006).

[294] H.A. Bethe, Z. Physik 21, 205 (1931).

[295] M. Karbach and G. Müller, Computers in Physics 11, 36 (1997) (cond-mat/9809162).

[296] M. Karbach, K. Hu, G. Müller and J. Tobochnik, Computers in Physics 12, 565 (1998).

[297] G. Vidal, Phys. Rev. Lett. 91, 147902 (2003).

[298] G. Vidal, J.I. Latorre, E. Rico, and A. Kitaev, Phys. Rev. Lett. 90, 227902 (2003).

[299] R. Orus and J.I. Lattore, Phys. Rev. A 69, 052308 (2004).

[300] F. Verstraete, D. Porras and J.I. Cirac, Phys. Rev. Lett 93, 227205 (2004).

[301] F. Verstraete, M.A. Martín-Delgado, and J.I. Cirac, Phys. Rev. Lett. 92, 087201 (2004).

[302] F. Verstraete, M. Popp and J.I Cirac, Phys. Rev. Lett. 92, 027901 (2004).

[303] S. Anders, M.B. Plenio, W. Dür, F. Verstraete, and H.-J. Briegel, Phys. Rev. Lett. 97, 107206 (2006).

[304] S.R. White, Phys. Rep. 301, 187 (1998).

[305] S.R. Clark and D. Jaksch, New J. Phys. 8, 160 (2006).

[306] F. Verstraete and J.I. Cirac, cond-mat/0407066.

[307] S. Östlund and S. Rommer, Phys. Rev. Lett. 75, 3537 (1995).

[308] S. Rommer and S. Östlund, Phys. Rev. B, 55, 2164 (1997).

[309] M. Fannes, B. Nachtergaele, R.F. Werner, Europhys. Lett. 10, 663 (1989).

[310] M. Fannes, B. Nachtergaele, and R.F. Werner, Comm. Math. Phys. 144, 441 (1992).

[311] F. Verstraete and J.I. Cirac, Phys. Rev. B 73, 094423 (2006).

[312] M.B. Hastings, Phys. Rev. Lett. 93, 140402 (2004).

[313] G. Vidal, cond-mat/0605597.

[314] F. Verstraete, M.M. Wolf, D. Perez-Garcia, J.I. Cirac, Phys. Rev. Lett. 96, 220601 (2006).

[315] N.F. Mott and W. D. Twose, Adv. Phys. 10, 107 (1961).

[316] R.E. Borland, Proc. R. Soc. London Ser. A 274, 529 (1963).

[317] N.F. Mott, J. Non-Cryst. Solids 1, 1 (1968).

[318] J.T. Edwards and D.J. Thouless, J. Phys. C 5, 807 (1972).

[319] D. J. Thouless, Phys. Rep. 13, 93 (1974).

[320] D. C. Licciardello and D. J. Thouless, J. Phys. C 8, 4157 (1975).

[321] F. J. Wegner, Z. Phys. 25, 327 (1976).

[322] E. Abrahams, P. W. Anderson, D. C. Licciardello and T. V. Ramakrishnan, Phys. Rev. Lett. 42, 673 (1979).

[323] L. P. Gorkov, A. I. Larkin and D. E. Khmelnitskii, JETP Lett. 30, 228 (1979)

[324] F. Haake, Quantum Signatures of Chaos, (Springer, Berlin, 2004).

[325] Y. Nagaoka and H. Fukuyama (Eds.), Anderson Localization, Springer Series in Solid State Sciences 39, (Springer, Heidelberg, 1982).

[326] P.A. Lee and R.V. Ramakrishan, Disordered electronic systems, Rev. Mod. Phys. 57, 287 (1985).

[327] T. Ando and H. Fukuyama (Eds.), Anderson Localization, Springer Proceedings in Physics 28, (Springer, Heidelberg, 1988).

[328] B. Kramer and A. MacKinnon, Rev. Prog. Phys. 56, 1469 (1993).

[329] N. E. Cusack, The Physics of Structurally Disordered Matter, (Adam Hilger, Bristol, 1987).

[330] R. Balian, R. Maynard, and G. Toulouse (Eds.), Ill-Condensed Matter, (North-Holland, Amsterdam, 1979).

[331] D. Chowdhury, Spin Glasses and other Frustrated Systems, (Wiley, New York, 1986).

[332] C. M. Newman and D. L. Stein, J. Phys.-Condens. Mat. 15, R1319 (2003).

[333] D. J. Amit, Modelling Brain Function, (Cambridge University Press, Cambridge, 1989).

[334] A. Aharony and D. Stauffer, Introduction to percolation Theory (Taylor \& Francis, London, 1994); G. Grimmett, Percolation (Springer, Berlin, 1999).

[335] P.H.R. Barbosa, E.P. Raposo, and M.D. Coutinho-Filho, Phys. Rev. Lett. 91, 197207 (2003).

[336] K. Efetov, Supersymmetry in Disorder and Chaos, (Cambridge University Press, Cambridge, 1997).

[337] B. Paredes, F. Verstraete, and J.I. Cirac, cond-mat/0505288.

[338] M. Ma, B.I. Halperin and P.A. Lee, Phys. Rev. B 34, 3136 (1986).

[339] T. Giamarchi and H. J. Schulz, Phys. Rev. B 37, 325 (1988).

[340] K.G. Singh and D.S. Rokhsar, Phys. Rev. B 46, 9013 (1992).

[341] L. Zhang and M. Ma, Phys. Rev. B 45, 4855 (1992).

[342] L. Zhang and X.-Q. Wang, Phys. Rev. B 47, 11518 (1993).

[343] L. Zhang, Phys. Rev. B 47, 14364 (1993). 
[344] D. K.K. Lee and J.M.F. Gunn, J. Phys. C: Condens. Matter 2, 7753 (1990).

345] K. Huang and H.-F. Meng, Phys. Rev. Lett. 69, 644 (1992).

346] K.G. Singh and D.S. Rokhsar, Phys. Rev. B 49, 9013 (1994).

[347] S. Giorgini, L. Pitaevskii and S. Stringari, Phys. Rev. B 49, 12938 (1994).

[348] A.V. Lopatin and V.M. Vinokur, Phys. Rev. Lett. 88, 235503 (2002).

[349] W. Krauth, N. Trivedi and D. Ceperley, Phys. Rev. Lett. 67, 2307 (1991).

[350] J. Kisker and H. Rieger, Phys. Rev. B 55, R11981 (1997).

351] F. Pázmándi, G. Zimaányi and R. Scalettar, Phys. Rev. Lett. 75, 1356 (1995).

[352] M. Wallin, E.S. Sørensen, S.M. Girvin and A.P. Young, Phys. Rev. B 49, 12115 (1994).

[353] N. Prokofév and B. Svistunov, Phys. Rev. Lett. 92, 015703 (2004).

[354] Y.B. Kim and X.-G. Wen, Phys. Rev. B 49, 4043 (1994).

[355] S. Rapsch, U. Schollwöck and W. Zwerger, Europhys. Lett. 46 (5), 559 (1999).

[356] L. Guidoni, C. Triché, P. Verkerk, and G. Grynberg, Phys. Rev. Lett. 79, 3363 (1997).

[357] L. Guidoni and P. Verkerk, Phys. Rev. A 57, R1501 (1998).

[358] L. Guidoni, B. Dépret, A. di Stefano, and P. Verkerk, Phys. Rev. A 60, R4233 (1999).

[359] L. Sanchez-Palencia and L. Santos, Phys. Rev. A 72, 053607 (2005).

[360] R.B. Diener, G.A. Georgakis, J. Zhong, M. Raizen and Q. Niu, Phys. Rev. A 64, 033416 (2001).

[361] J.W. Goodman, in Laser Speckle and Related Phenomena, edited by J.C. Dainty (Springer, Berlin, 1975).

[362] P. Horak, J.-Y. Courtois, and G. Grynberg, Phys. Rev. A 58, 3953 (1998).

[363] D. Boiron, C. Mennerat-Robilliard, J.-M. Fournier, L. Guidoni, C. Salomon and G. Grynberg, Eur. Phys. J. D 7, 373 (1999).

[364] U. Gavish and Y. Castin, Phys. Rev. Lett. 95, 020401 (2005).

[365] P. Zoller, H.P. Büchler, and I. Bloch (private communication).

[366] N. Kawashima and H. Rieger, Recent progress in spin glasses (cond-mat/0312432) in Frustrated spin systems, edited by H.T. Diep (World Scientific, Singapore, 2004).

[367] A. Kantian, Disordered Quantum Phases in Optical Lattices, Diploma Thesis, Universität Hannover, 2004.

[368] H. Gimperlein, S. Wessel, J. Schmiedmayer, and L. Santos, Phys. Rev. Lett. 95, 170401 (2005).

[369] R. Folman, P. Krüger, J. Schmiedmayer, J. Denschlag, and C. Henkel, Adv. At. Mol. Opt. Phys. 48, 263 (2002). For the latest results see Y.J. Wang, D.Z. Anderson, V.M. Bright, E.A. Cornell, Q. Diot, T. Kishimoto, M. Prentiss, R.A. Saravanan, S.R. Segal, and S.J. Wu, Phys. Rev. Lett. 94, 090405 (2005); T. Schumm, J. Esteve, C. Figl, J.B. Trebbia, C. Aussibal, H. Nguyen, D. Mailly, I. Bouchoule, C.I. Westbrook, and A. Aspect, Eur. Phys. J. D 32, 171 (2005); J. Fortagh and C. Zimmermann, Science 307, 860 (2005); P. Hommelhoff, W. Hansel, T. Steinmetz, T. W. Hänsch, and J. Reichel, New J. Phys. 7, Art. no. 3, Jan. (2005); L. Feenstra, L.M. Andersson, and J. Schmiedmayer, Gen. Rel. Grav. 36, 2317 (2004).

[370] C. Henkel, P. Krüger, R. Folman, and J. Schmiedmayer, Appl. Phys. B 76, 173 (2003).

[371] D.-W. Wang, M. D. Lukin, and E. Demler, Phys. Rev. Lett. 92, 076802 (2004).

[372] M.G. Raizen (private communication).

[373] A. Lenard, J. Math. Phys. 5, 930 (1964).

[374] H.G. Vaidya and C.A. Tracy, Phys. Rev. Lett. 42, 3 (1979).

[375] D.M. Gangardt, J. Phys. A 37, 9335 (2004).

[376] T. Cheon and T. Shigehara, Phys. Rev. Lett. 82, 2536 (1999).

[377] K.V. Krutitsky, A. Pelster, and R. Graham, New J. Phys. 8, 187 (2006).

[378] V.W. Scarola and S. Das Sarma, Phys. Rev. A 73, 041609(R) (2006).

[379] S. Richard, F. Gerbier, J.H. Thywissen, M. Hugbart, P. Bouyer, and A. Aspect, Phys. Rev. Lett. 91, 010405 (2003).

[380] L. Fallani, L. De Sarlo, J.E. Lye, M. Modugno, R. Saers, C. Fort and M. Inguscio, Phys. Rev. Lett. 93, 140406 (2004).

[381] Yu. Kagan, E.L. Surkov and G.V. Schlyapnikov, Phys. Rev. A 54, R1753 (1994).

[382] Y. Castin and R. Dum, Phys. Rev. Lett. 77, 5315 (1996).

[383] B. De Marco and D.S. Jin, Science 285, 1703 (1999).

[384] A.G. Truscott, K.E. Strecker, W.I. McAlexander, G.B. Partridge, and R.G. Hulet, Science 291, 2570 (2001).

[385] F. Schreck, L. Khaykovich, K.L. Corwin, G. Ferrari, T. Bourdel, J. Cubizolles, and C. Salomon, Phys. Rev. Lett. 87, 80403 (2001).

[386] Z. Hadzibabic, C.A. Stan, K. Dieckmann, S. Gupta, M.W. Zwierlein, A. Görlitz, and W. 
Ketterle, Phys. Rev. Lett. 88, 160401 (2002).

[387] G. Roati, F. Riboli, G. Modugno and M. Inguscio, Phys. Rev. Lett. 89, 150403 (2002).

[388] C. Honerkamp and W. Hofstetter, Phys. Rev. Lett. 92, 170403 (2004).

[389] B. Paredes, C. Tejedor and J.I. Cirac, Phys. Rev. A 71, 063608 (2005).

[390] P.W. Anderson, Comments Solid State Phys. 2, 193 (1970).

[391] R. Freedman and J.A. Hertz, Phys. Rev. B 15, 2384 (1977)

[392] B.L. Altshuler and A.G. Aronov, Solid State Comm. 30, 115 (1079).

[393] B.L. Altshuler and A.G. Aronov, Sov. Phys. JETP 50, 968 (1979).

[394] B.L. Altshuler, A.G. Aronov, and P.A. Lee, Phys. Rev. Lett. 44, 1288 (1980).

[395] H. Fukuyama, J. Phys. Soc. Jpn. 48, 2169 (1980).

[396] D.L. Shepelyansky, Phys. Rev. Lett. 73, 2607 (1994).

[397] Y. Imry, Europhys. Lett. 30, 405 (1995).

[398] K. Frahm, A. Müller-Groeling, J.L. Pichard and D. Weinmann, Europhys. Lett. 31, 405 (1995).

[399] D. Weinmann, A. Müller-Groeling, J. L. Pichard and K. Frahm, Phys. Rev. Lett. 75, 1598 (1995).

[400] F. von Oppen, T. Wettig and J. Müller, Phys. Rev. Lett. 76, 491 (1996).

[401] Correlated Fermions and Transport in Mesoscopic systems, edited by T. Martin, G. Montambaux, and J. Tran Thanh Van (Editions Frontieres, Gif-sur-Yvette, 1996).

[402] Ph. Jacquod and D.L. Shepelyansky, Phys. Rev. Lett. 78, 4986 (1997).

[403] P. Schmitteckert, R.A. Jalabert, D. Weinmann and J.-L. Pichard, Phys. Rev. Lett. 81, 2308 (1998).

[404] E. Gambeti-Césare, D. Weinmann, R.A. Jalabert, and Ph. Brune, Europhys. Lett. 60, 120 (2002).

[405] G. Benenti, X. Waintal, and J.-L. Pichard, Phys. Rev. Lett. 83, 1826 (1999).

[406] X. Waintal, G. Benenti, and J.-L. Pichard, Europhys. Lett. 49, 466 (2000).

[407] A.L. Efros and B.I. Shklovskii, J. Phys. C 8, L49 (1975).

[408] A.L. Efros and B.I. Shklovskii, Phys. Stat. Sol. B 76, 475 (1976).

[409] A.L. Efros, Sov. Phys. JETP 54, 218 (1981).

[410] W.L. McMillan, Phys. Rev. B 24, 2739 (1981).

[411] H.-L. Lee, J.P. Carini, D.V. Baxter, and G. Gruner, Phys. Rev. Lett. 80, 4261 (1998).

[412] H.-L. Lee, J.P. Carini, D.V. Baxter, W. Henderson, and G. Gruner, Science 287, 633 (2000).

[413] M.A. Paalanen, T.F. Rosenbaum, G.A. Thomas, and R.N. Bhatt, Phys. Rev. Lett. 51, 1896 (1983).

[414] S. Bogdanovich, M.P. Sarachik, and R.N. Bhatt, Phys. Rev. Lett. 82, 137 (1999).

[415] J.H. Davies, P.A. Lee, and T.M. Rice, Phys. Rev. Lett. 49, 758 (1982).

[416] J. H. Davies, P. A. Lee, T. M. Rice, Phys. Rev. B 29, 4260 (1984).

[417] M. Pollak and M. Ortuño, .Electron-Electron interactions in disordered systems edited by A. L. Efros and M. Pollak (North-Holland, Amsterdam, 1985) p.287.

[418] M. Hering, M. Scheffler, M. Dressel, and H. Von Lohneysen, Physica B 359, 1469 (2005).

[419] M. Scheffler, M. Dressel, M. Jourdan and H. Adrian, Nature 438, 1135 (2005).

[420] K. Byczuk, W. Hofstetter and D. Vollhardt, Phys. Rev. Lett. 94, 056404 (2005).

[421] K. Byczuk, W. Hofstetter and D. Vollhardt, Physica B 359-361, 651 (2005).

[422] H. Ott, E. de Mirandes, F. Ferlaino, G. Roati, G. Modugno, and M. Inguscio, Phys. Rev. Lett. 92, 160601 (2004).

[423] R. Roth and K. Burnett, Phys. Rev. A 69, 021601(R) (2004).

[424] R. Roth and K. Burnett, J. Phys. B 37, 3893 (2004).

[425] D. Sherrington, cond-mat/9806289.

[426] D.S. Fisher and D.A. Huse, Phys. Rev. B 38, 373 (1988).

[427] D.S. Fisher and D.A. Huse, Phys. Rev. B 38, 386 (1988).

[428] R. Oppermann and B. Rosenow, Phys. Rev. B 60, 10325 (1999).

[429] R. Oppermann and D. Sherrington, Phys. Rev. B 67, 245111 (2003).

[430] K. Binder, A.P. Young, Rev. Mod. Phys. 58, 801 (1986)

[431] A. Georges, O. Parcollet, and S. Sachdev, Phys. Rev. B 63, 134406 (2001).

[432] A.P. Young and H.G. Katzgraber, Phys. Rev. Lett. 93, 207203 (2004).

[433] H.G. Katzgraber and A.P. Young, Phys. Rev. B 72, 184416 (2005).

[434] F. Krzakala and J-P. Bouchaud, Europhys. Lett. 72, 472 (2005).

[435] H.G. Katzgraber, L.W. Lee, and I.A. Campbell, cond-mat/0510668.

[436] H.G. Katzgraber, M. Körner, and A.P. Young, Phys. Rev. B, 73, 224432 (2006).

[437] H.P. Büchler, Workshop on disordered ultracold atomic gases, Hannover 2004. 
[438] R. Moesner and S.L. Sondhi, Phys. Rev. B 63, 224401 (2001).

[439] M. Rasolt and Z. Tesanović, Rev. Mod. Phys. 64, 709 (1992)

[440] M. Sigrist and T.M. Rice, Rev. Mod. Phys. 67, 503 (1995).

[441] B. Bernu, C. Lhuillier, and L. Pierre, Phys. Rev. Lett. 69, 2590 (1992); B. Bernu, P. Lecheminant, C. Lhuillier, and L. Pierre, Phys. Rev. B 50, 10048 (1994).

[442] X.-G. Wen, Quantum Field Theory of Many-body Systems, Oxford University Press (2004).

[443] P.W. Anderson, Science, 237, 1196 (1987).

[444] S. Liang, B. Daucot, and P.W. Anderson, Phys. Rev. Lett. 61, 365 (1988).

[445] E. Manousakis, Rev. Mod. Phys. 63, 1 (1991).

[446] L. Hulthén, Arkiv. Mat. Astron. Physik, 26A, 11 (1938).

[447] C.N. Yang and C.P. Yang, Phys. Rev. 150, 321 (1966); ibid. 150, 327 (1966).

[448] F.D.M. Haldane, Phys. Lett 93A, 464 (1983); Phys. Rev. Lett. 50, 1153 (1983).

[449] J.C. Bonner, J. Appl. Phys. 61, 3941 (1987).

[450] R. Melzi, P. Carretta, A. Lascialfari, M. Mambrini, M. Troyer, P. Millet, and F. Mila, Phys. Rev. Lett. 85, 1318 (2000).

451] C.K. Majumdar and D.K. Ghosh, J. Math. Phys. 10, 1388 (1969).

[452] I. Bose and P. Mitra, Phys. Rev. B 44, 443(R) (1991).

[453] U. Bhaumik and I. Bose, Phys. Rev. B 52, 12489 (1995).

454] B. Kumar, Phys. Rev. B 66, 024406 (2002)

[455] N. Surendran and R. Shankar, Phys. Rev. B 66, 024415 (2002).

[456] E. Dagotto and A. Moreo, Phys. Rev. Lett. 63, 2148 (1989).

457] R.P. Singh and R. Narayanan, Phys. Rev. Lett. 65, 1072 (1990).

[458] H.A. Ceccatto, C.J. Gazza, and A.E. Trumper, Phys. Rev. B 45, 7832 (1992).

[459] J. Richter, Phys. Rev. B 47, 5794 (1993).

[460] J. Oitmaa and Z. Weihong, Phys. Rev. B 54, 3022 (1996).

[461] R.P. Singh, M.P. Gelfand, and D.A. Huse, Phys. Rev. Lett. 61, 2484 (1989).

462 M.P. Gelfand, R.P. Singh, and D.A. Huse, J. Stat. Phys. 59, 1093 (1990).

[463] E.F. Shender and P.C.W. Holdsworth, Fluctuations and Order: The New Synthesis (edited by M.M. Millonas, MIT Press, 1994).

[464] C.L. Henley, Phys. Rev. Lett. 62, 2056 (1989).

[465] P.W. Anderson, Phys. Rev. 83, 1260 (1951).

[466] R. Kubo, Phys. Rev. 87, 568 (1952).

467] T. Oguchi, Phys. Rev. 117, 117 (1960).

[468] D. Poilblanc, E. Gagliano, S. Bacci, and E. Dagatto, Phys. Rev. B 43, 10970 (1991).

[469] H.J. Schulz and T.A.L. Ziman, Europhys. Lett. 18, 355 (1992)

[470] H.J. Schulz and T.A.L. Ziman, Phys. Rev. B 51, 6151 (1995).

[471] A.W. Sandvik, Phys. Rev. B 56, 11678 (1997) and references therein.

[472] F. Figueirido, A. Carlhede, S. Kivelson, S. Sondhi, M. Rocek, and D.S. Rokhsar, Phys. Rev. B 41, 2148 (1990).

[473] H.J. Schulz, T.A.L. Ziman, and D. Poilblanc, J. Phys. I 6, 675 (1996)

[474] N. Read and S. Sachdev, Phys. Rev. Lett. 62, 1694 (1989).

[475] N. Read and S. Sachdev, Phys. Rev. Lett. 66, 1773 (1991).

[476] G. Murthy and S. Sachdev, Nucl. Phys. B, 344, 557 (1990).

[477] V.N. Kotov, O.P. Sushkov, J. Oitmaa, and Z. Weihong, Phys. Rev. B 60, 14613 (1999).

[478] M.P. Gelfand, R.R.P. Singh, and D.A. Huse, Phys. Rev. B 40, 10801 (1989).

[479] M.P. Gelfand, Phys. Rev. B 42, 8206 (1990).

480] R.R.P. Singh, Z. Weihong, C.J. Hammer, and J. Oitmaa, Phys. Rev. B 60, 7278 (1999).

[481] L. Capriotti, F. Becca, A. Parola, and S. Sorella, Phys. Rev. Lett. 87, 097201 (2001).

482] O.P. Sushkov, J. Oitmaa, and Z. Weihong, Phys. Rev. B 63, 104420 (2001).

[483] R. Moesner, S.L. Sondhi, and P. Chandra, Phys. Rev. Lett. 84, 4457 (2000).

[484] C. Waldtmann, H.-U. Everts, B. Bernu, C. Lhuillier, P. Sindzingre, P.Lecheminant, L. Pierre, Eur. Phys. J. B 2, 501 (1998).

[485] C. Zeng and V. Elser, Phys. Rev. B 42, 8436 (1990).

[486] R. Singh and D. Huse, Phys. Rev. Lett. 68, 1766 (1992).

[487] P. Leung and V. Elser, Phys. Rev. B 47, 5459 (1993).

[488] C. Zeng and V. Elser, Phys. Rev. B 51, 8318 (1995).

[489] P. Sindzingre, P. Lecheminant and C. Lhuillier, Phys. Rev. B 50, 3108 (1994).

[490] T. Nakamura and S. Miyashita, Phys. Rev. B 52, 9174 (1995).

[491] P. Lecheminant, B. Bernu, C. Lhuillier, L. Pierre, and P. Sindzingre, Phys. Rev. B 56, 2521 (1997). 
[492] F. Mila, Phys. Rev. Lett. 81, 2356 (1998).

[493] M. Mambrini and F. Mila, Eur. Phys. J. B 17, 651 (2001).

[494] R. Budnik and A. Auerbach, Phys. Rev. Lett. 93, 187205 (2004).

[495] D. Porras and J.I. Cirac, Phys. Rev. Lett. 92, 207901 (2004)

[496] X.-L. Deng, D. Porras, and J.I. Cirac, Phys. Rev. A 72, 063407 (2004).

[497] D. Porras and J.I. Cirac, quant-ph/0601148.

[498] H.J. Briegel and R. Raussendorf, Phys. Rev. Lett. 86, 910 (2001).

[499] R. Raussendorf and H.-J. Briegel, Phys. Rev. Lett. 86, 5188 (2001).

[500] A. Kitaev, Ann. Phys. 321, 2 (2006).

[501] F. Wilczek, Phys. Rev. Lett. 48, 1144 (1982).

[502] F. Wilczek, Phys. Rev. Lett. 48, 1146 (1982).

[503] T. Rieger, T. Junglen, S.A. Rangwala, P.W.H. Pinkse, and G. Rempe, Phys. Rev. Lett. 95, 173002 (2005).

[504] F. Mintert and C. Wunderlich, Phys. Rev. Lett. 87, 257904 (2001).

[505] W.M. Itano, J.J. Bollinger, J.N. Tan, B. Jelenković, X.-P. Huang, and D.J. Wineland, Science 279, 686 (1998).

[506] T.B. Mitchell, J.J. Bollinger, D.H.E. Dubin, X.-P. Huang, W.M. Itano, and R.H. Baughman, Science 282, 1290 (1998).

[507] C.J. Myatt, E.A. Burt, R.W. Ghrist, E.A. Cornell, and C.E. Wieman, Phys. Rev. Lett. 78, 586 (1997); D.S. Hall, M.R. Matthews, J.R. Ensher, C.E. Wieman, and E.A. Cornell, ibid. 81, 1539 (1998); M.R. Matthews, D.S. Hall, D.S. Jin, J.R. Ensher, C.E. Wieman, E.A. Cornell, F. Dalfovo, C. Minniti, and S. Stringari, ibid. 81, 243 (1998); J.M. McGuirk, H.J. Lewandowski, D.M. Harber, T. Nikuni, J.E. Williams, and E.A. Cornell, Phys. Rev. Lett. 89, 090402 (2002); H.J. Lewandowski, J.M. McGuirk, D.M. Harber, and E.A. Cornell, Phys. Rev. Lett. 91, 240404 (2003).

[508] D.M. Stamper-Kurn and W. Ketterle, Proceedings of Les Houches 1999 Summer School, Session LXXII.

[509] T.-L. Ho, Phys. Rev. Lett. 81, 742 (1998).

[510] M. Koashi and M. Ueda, Phys. Rev. Lett. 84, 1066 (2000).

[511] J. Stenger, S. Inouye, D.M. Stamper-Kurn, H.J. Miesner, A.P. Chikkatur, and W. Ketterle, Nature 396, 345 (1998).

[512] T. Ohmi and K. Machida, J. Phys. Soc. Jpn. 67, 1822 (1998); T. Isoshima, K. Machida, and T. Ohmi, Phys. Rev. A 60, 4857 (1999).

[513] W. Zhang, S. Yi, and L. You, Phys. Rev. A 70, 043611 (2004).

[514] C.V. Ciobanu, S.K. Yip, and T.-L. Ho, Phys. Rev. A 61, 033607 (2001).

[515] M. Ueda and M. Koashi, Phys. Rev. A 65, 063602 (2002).

[516] R.B. Diener and T.-L. Ho, Phys. Rev. Lett. 96, 190405 (2006).

[517] L. Santos and T. Pfau, cond-mat/0510634.

[518] M.D. Barrett, J.A. Sauer, and M.S. Chapman, Phys. Rev. Lett. 87, 010404 (2001).

[519] H. Schmaljohann, M. Erhard, J. Kronjager, M. Kottke, S. van Staa, L. Cacciapuoti, J.J. Arlt, K. Bongs, and K. Sengstock, Phys. Rev. Lett. 92, 040402 (2004).

[520] M.S. Chang, C.D. Hamley, M.D. Barrett, J.A. Sauer, K.M. Fortier, W. Zhang, L. You, and M.S. Chapman, Phys. Rev. Lett. 92, 140403 (2004).

[521] T. Kuwamoto, K. Araki, T. Eno, and T. Hirano, Phys. Rev. A 69, 063604 (2004).

[522] M. Erhard, H. Schmaljohann, J. Kronjäger, K. Bongs, and K. Sengstock, Phys. Rev. A 70, 031602 (2004).

[523] J.M. Higbie, L.E. Sadler, S. Inouye, A.P. Chikkatur, S.R. Leslie, K.L. Moore, V. Savalli, and D.M. Stamper-Kurn Phys. Rev. Lett. 95, 050401 (2005).

[524] A. Widera, F. Gerbier, S. Folling, T. Gericke, O. Mandel, and I. Bloch, Phys. Rev. Lett. 95 190405 (2005).

[525] M. Rizzi, D. Rossini, G. De Chiara, S. Montangero, and R. Fazio, Phys Rev. Lett. 95, 240404 (2005).

[526] D. Porras, F. Verstraete, and J.I. Cirac, cond-mat/0504717.

[527] O. Romero-Isart, K. Eckert, and A. Sanpera, quant-ph/0610210.

[528] K.V. Krutitsky and R. Graham, Phys. Rev. Lett. 91, 240406 (2003;) K.V. Krutitsky and R. Graham, Phys. Rev. A 70, 063610 (2004); K.V. Krutitsky, M. Timmer, and R. Graham, Phys. Rev. A 71, 033623 (2005).

[529] T. Kimura, S. Tsuchiya and S. Kurihara, Phys. Rev. Lett. 94, 110403 (2005)

[530] M.M. Wolf, G. Ortiz, F. Verstraete, and J.I. Cirac, cond-mat/0512180.

[531] T. Paananen, J.-P. Martikainen, and P. Torma, Phys. Rev. A 73, 053606 (2006). 
[532] For a review, see W. Hofstetter, in print in Adv. Solid State Phys., cond-mat/0504113.

[533] T. Weber, J. Herbig, M. Mark, H.C. Nagerl, and R. Grimm, Science 299, 232 (2003).

[534] Y. Takahashi, Y. Takasu, K. Maki, K. Komori, T. Takano, K. Honda, A. Yamaguchi, Y. Kato, M. Mizoguchi, M. Kumakura, and T. Yabuzaki, Laser Phys. 14, 621 (2004).

[535] H.-H. Tu, G.-M. Zhang, and L. Yu, Phys. Rev. B 74, 174404 (2006).

536] C. Wu, J.-P. Hu, and S.-C. Zhang, submitted to Phys. Rev. Lett. (cond-mat/0512602).

[537] C. Wu, J.-P. Hu, and S.-C. Zhang, Phys. Rev. Lett. 91, 186402 (2003).

538] C. Wu, Phys. Rev. Lett. 95, 266404 (2005).

[539] C. Wu, cond-mat/0608690.

[540] R.F. Prange and S.M. Girvin, The Quantum Hall Effect, (Springer, Berlin, 1987).

[541] K.W. Madison, F. Chevy, W. Wohlleben and J. Dalibard, Phys. Rev. Lett 84, 806 (2000).

[542] J.R. Abo-Shaeer, C. Raman, J.M. Vogels, and W. Ketterle, Science 292, 476 (2001).

[543] N.K. Wilkin, J.M.F. Gunn, and R.A. Smith, Phys. Rev. Lett 80, 2265 (1998).

[544] M.A. Cazalilla, N. Barberan, and N.R. Cooper, Phys. Rev. A 71, 121303(R) (2005).

[545] J. Sinova, C.B. Hanna, A.H. MacDonald, Phys. Rev. Lett 89, 030403 (2002)

546] N. Regnault and T. Jolicoeur, Phys. Rev. B 18, 1003 (2004).

547] N. Regnault and T. Jolicoeur, Phys. Rev. B 70, 241307 (2004).

[548] N. Read and E. Rezayi, Phys. Rev. B 59, 8084 (1999).

549] G. Moore and N. Read, Nucl. Phys. B 360, 362 (1991).

[550] E.H. Rezayi, N. Read and N.R. Cooper, Phys. Rev. Lett 95, 160404 (2005).

[551] A.G. Morris and D.L. Feder, Phys. Rev. A 74, 033605 (2006).

[552] N. Regnault, C.C. Chang, Th. Jolicoeur, and J.K. Jain, J. Phys. B: At. Mol. Opt. Phys. 39, S89 (2006).

[553] I. Coddington, P. Engels, V. Schweikhard, and E.A. Cornell, Phys. Rev. Lett. 91, 100402 (2004).

[554] V. Schweikhard, I. Coddington, P. Engels, S. Tung, and E.A. Cornell, Phys. Rev. Lett. 93, 210403 (2004)

[555] V. Schweikhard, I. Coddington, P. Engels, V.P. Mogendorff, and E.A. Cornell, Phys. Rev. Lett. 92, 040404 (2002).

[556] T.P. Simula, P. Engels, I. Coddington, V. Schweikhard, E.A. Cornell, and R.J. Ballagh Phys. Rev. Lett. 94080404 (2005).

[557] S. Stock, B. Battelier, V. Bretin, Z. Hadzibabic, J. Dalibard, Laser Phys. Lett. 2 (6), 275 (2005).

[558] See eg. special issue on ultracold polar molecules [Eur. Phys. J. D 31, 149 (2004).

[559] G. Juzeliunas and P. Öhberg, Phys. Rev. Lett. 93, 033602 (2004).

[560] G. Juzeliunas, P. Öhberg, J. Ruseckas, and A. Klein, Phys. Rev. A 71, 053614 (2005).

[561] G. Juzeliunas, J. Ruseckas, and P. Öhberg, J. Phys. B: At. Mol. Opt. Phys. 38, 4171 (2005).

[562] G. Juzeliunas, J. Ruseckas, P. Öhberg, and M. Fleischhauer, quant-ph/0511226.

[563] P. Öhberg, G. Juzeliunas, J. Ruseckas, and M. Fleischhauer, Phys. Rev. A 71, 053632 (2005).

[564] I. Bloch, private communication.

[565] G. Birkl, private communication; see also G. Birkl, F.B.J. Buchkremer, R. Dumke, and W. Ertmer, Opt. Commun. 191, 1 (2001).

[566] D. D. Yavuz, P. B. Kulatunga, E. Urban, T. A. Johnson, N. Proite, T. Henage, T. G. Walker, and M. Saffman, Phys. Rev. Lett. 96, 063001 (2006).

[567] I. Romanovsky, C. Yannouleas, and U. Landman, Phys. Rev. Lett. 93, 230405 (2004); C. Yannouleas and Uzi Landman, Phys. Rev. B 70, 235319 (2004); I. Romanovsky, C. Yannouleas, L.O. Baksmaty, and U. Landman, Phys. Rev. Lett. 97090401 (2006).

[568] D. R. Hofstadter, Phys. Rev. B 14, 2239 (1976).

[569] P. G. Harper, Proc. Phys. Soc. Lond. A 68, 874 (1955).

[570] Z.F. Ezawa, Quantum Hall efects: field theoretical approach and related topics, (World Scientific, Singapore, 2000).

[571] A.A. Burkov and E. Demler, Phys. Rev. Lett. 96, 180406 (2006).

[572] M. Polini, R. Fazio, A.H. MacDonald, and M.P. Tosi, Phys. Rev. Lett. 95, 010401 (2005). See also M. Polini, R. Fazio, M.P. Tosi, J. Sinova, and A.H. MacDonald, Laser Phys. 14, 603 (2004).

[573] R. Bhat, M.J. Holland, and L.D. Carr, Phys. Rev. Lett. 96, 060405 (2006).

[574] C. Wu, H.-D. Chen, J.-P. Hu, S.-C. Zhang, Phys. Rev. A 69, 43609 (2004).

[575] H.J. Rothe, Lattice Gauge Theories (World Scientific, Singapore, 1998).

[576] Indubala I. Satija, D.C. Dakin, and C.W. Clark, cond-mat/0607246.

[577] N. Goldman and P. Gaspard, cond-mat/0609472. 
[578] For a very good pedagogical review of Yang-Mills systems in $(2+1)$ D, see H. Schulz, hep$\mathrm{ph} / 0008239$.

[579] A. Jacob and L. Santos, AMOP-Frühjahrstagung der DPG, Ver. Deutsch. Phys. Ges. 7/2006, Q2.4 (2006).

[580] A. Shapere and F. Wilczek, Geometric Phases in Physics, (World Scientific, Singapore, 1989).

[581] N. Read and G. Moore, Prog. Theor. Phys. Suppl. 107157 (1992) (hep-th/9202001).

[582] E. Ardonne and K. Schoutens, cond-mat/0606217.

[583] N. Read and E. Rezayi, Phys. Rev. B 54, 16864 (1996).

[584] M. Greiter, X.-G. Wen, and F. Wilczek Phys. Rev. Lett. 66, 3205 (1991).

[585] S. Das Sarma and A. Pinczuk (Eds.), Perspectives in Quantum Hall Effects: Novel Quantum Liquids in Low-Dimensional Semiconductor Structures, (Wiley \& Sons, New York, 1997)

[586] Understanding the Fractional Quantum Hall Effect without the Pfaffian Wave Function C. Töke and J.K. Jain, Phys. Rev. Lett. 96, 246805 (2006).

[587] B. Paredes, T. Keilmann, J. I. Cirac, cond-mat/0608012.

[588] For a recent review of LGT's, see M. Engelhardt, hep-lat/0409023.

[589] J. Greensite, Prog. Part. Nucl. Phys. 51, 1 (2003).

[590] M. Baig and J. Colet, Phys. Lett. B 176, 149 (1986).

[591] T. Senthil, A. Vishwanath, L. Balents, S. Sachdev, and M.P.A. Fisher, Science 303, 1490 (2004).

[592] M. Hemerle, M.P.A. Fisher, and L. Balents, Phys. Rev. B 69, 064404 (2004).

[593] M. Snoek, S. Vandoren, and H.T.C. Stoof, cond-mat/0604671.

[594] T.J. Osborne and M.A. Nielsen, Quantum Information Processing, 1, 45 (2002) (quant$\mathrm{ph} / 0109024)$

[595] T.J. Osborne and M.A. Nielsen, Phys. Rev. A 66, 032110 (2002).

[596] A. Osterloh, L. Amico, G. Falci, and R. Fazio, Nature 416, 608 (2002).

[597] R.F. Werner, Phys. Rev. A 40, 4277 (1989)

[598] A. Sen(De), U. Sen, M. Lewenstein, and A. Sanpera, The separability versus entanglement problem, in Lectures on Quantum Information, eds. D. Bruss and G. Leuchs (Wiley-VCH, 2006), to be published (ISBN: 3527405275). The chapter is available at quant-ph/0508032.

[599] A.C. Doherty, P.A. Parrilo, and F.M. Spedelieri, Phys. Rev. Lett. 88, 187904 (2002).

[600] F. Hulpke and D. Bruß, J. Phys. A: Math. Gen. 38, 5573 (2005).

[601] A. Peres, Phys. Rev. Lett 77, 1413 (1996).

[602] M. Horodecki, P. Horodecki, and R. Horodecki, Phys. Lett. A 223, 1 (1996).

[603] C.H. Bennett, D.P. DiVincenzo, J.A. Smolin, and W.K. Wootters, Phys. Rev. A 54, 3824 (1996).

[604] V. Vedral, M.B. Plenio, M.A. Rippin, and P.L. Knight, Phys. Rev. Lett 78, 2275 (1997).

[605] D.P. DiVincenzo, C.A. Fuchs, H. Mabuchi, J.A. Smolin, A. Thapliyal, A. Uhlmann, quant$\mathrm{ph} / 9803033$.

[606] T. Laustsen, F. Verstraete, and S.J. van Enk, Quantum Inf. Comput. 3, 64 (2003).

[607] M.A. Nielsen, Phys. Rev. Lett. 83, 436 (1999).

[608] G. Vidal, J. Mod. Opt. 47, 355 (2000).

[609] D. Jonathan and M.B. Plenio, Phys. Rev. Lett. 831455 (1999).

[610] M. Horodecki, A. Sen(De), and U. Sen, Phys. Rev. A 70, 052326 (2004);

[611] M. Horodecki, Quantum Inf. Comput. 1, 7 (2001).

[612] M.B. Plenio and S. Virmani, Quant. Inf. Comp. 7, 1 (2007) (quant-ph/0504163)

[613] C.H. Bennett, H. Bernstein, S. Popescu, and B. Schumacher, Phys. Rev. A 53, 2046 (1996).

[614] S. Hill and W.K. Wootters, Phys. Rev. Lett. 78, 5022 (1997).

[615] W.K. Wootters, Phys. Rev. Lett. 80, 2245 (1998).

[616] B.M. Terhal and K.G.H. Vollbrecht, Phys. Rev. Lett. 85, 2625 (2000)

[617] G. Vidal, W. Dür, and J.I. Cirac, Phys. Rev. Lett. 89, 0279012625 (2002).

[618] M. Horodecki, A. Sen(De), and U. Sen, Phys. Rev. A 67, 062314 (2003).

[619] G. Giedke, M.M. Wolf, O. Krüger, R.F. Werner, and J.I. Cirac, Phys. Rev. Lett. 91, 107901 (2003).

[620] M.M. Wolf, G. Giedke, O. Krüger, R.F. Werner, and J.I. Cirac, Phys. Rev. A 69, 052320 (2004).

[621] G. Vidal and R.F. Werner, Phys. Rev. A 65, 032314 (2002).

[622] A. Sanpera, R. Tarrach, and G. Vidal, Phys. Rev. A 58, 826 (1998).

[623] K. Audenaert, M.B. Plenio, and J. Eisert, Phys. Rev. Lett. 90, 027901 (2003).

[624] M.B. Plenio, Phys. Rev. Lett. 95, 090503 (2005) 
[625] V. Coffman, J. Kundu, and W.K. Wootters, Phys. Rev. A 61, 052306 (2000).

[626] M.A. Nielsen, Ph.D. thesis, University of New Mexico, USA, 1998 (quant-ph/0011036).

[627] J. Preskill, J. Mod. Phys. 47, 127 (2000).

[628] X. Wang, Phys. Rev. A 64, 012313 (2001).

629] K.A. Dennison and W.K. Wootters, quant-ph/0106058.

[630] X. Wang, H. Fu, and A.I. Solomon, J. Phys. A 34, 11307 (2001).

[631] K.M. O'Connors and W.K. Wootters, Phys. Rev. A 63, 052302 (2001).

[632] M.C. Arnesen, S. Bose, and V. Vedral, Phys. Rev. Lett. 87, 017901 (2001).

[633] D. Gunlycke, S. Bose, V.M. Kendon, and V. Vedral, Phys. Rev. A 64, 042302 (2001).

[634] D.A. Meyer and N.R. Wallach, quant-ph/0108104.

[635] X. Wang, Phys. Lett. A 281, 101 (2001).

[636] W.K. Wootters, quant-ph/0202048.

[637] W.K. Wootters, Contemporary Mathematics 305, 299 (2002).

[638] P. Zanardi, Phys. Rev. A 65, 042101 (2002).

[639] P. Zanardi and X. Wang, J. Phys. A 35, 7947 (2002).

[640] I. Bose and E. Chattopadhyay, Phys. Rev. A 66, 062320 (2002).

[641] H. Fu, A.I. Solomon, and X. Wang, quant-ph/0201025.

[642] X. Wang and P. Zanardi, Phys. Lett. A 301, 1 (2002).

[643] S.C. Benjamin and S. Bose, Phys. Rev. Lett. 90, 247901 (2003).

[644] S. Bose, Phys. Rev. Lett. 91, 207901 (2003).

[645] T. Roschilde, P. Verrucchi, A. Fubini, S. Haas, and V. Tognetti, Phys. Rev. Lett. 93, 167203 (2004).

[646] A. Anfossi, P. Giorda, A. Montorsi, and F. Traversa, Phys. Rev. Lett. 95, 056402 (2005).

[647] T. Roschilde, P. Verrucchi, A. Fubini, S. Haas, and V. Tognetti, Phys. Rev. Lett. 94, 147208 (2005).

[648] T.-C. Wei, D. Das, S. Mukhopadyay, S. Vishveshwara, and P.M. Goldbart, Phys. Rev. A 71, 060305(R) (2005)

[649] T.R. de Oliveira, G. Rigolin, and M.C. de Oliveira, Phys. Rev. A 73, 010305(R) (2006).

[650] V.E. Korepin, Phys. Rev. Lett. 92, 096402 (2004).

[651] S.-J. Gu, S.-S. Deng, Y.-Q. Li, and H.-Q. Lin, Phys. Rev. Lett. 93, 086402 (2004)

[652] D. Larsson and H. Johannesson, Phys. Rev. Lett. 95, 196406 (2005).

[653] N. Lambert, C. Emary, and T. Brandes, Phys. Rev. Lett. 92, 073602 (2004).

[654] J. Eisert and M. Cramer, Phys. Rev. A 72, 042112 (2005).

[655] A. Sen(De), U. Sen, and M. Lewenstein, Phys. Rev. A 72, 052319 (2005).

656] E. Lieb, T. Schultz, and D. Mattis, Ann. Phys. 60, 407 (1961).

[657] B.M. McCoy, Phys. Rev. 173, 531 (1968).

[658] P. Pfeuty, Ann. Phys. 57, 79 (1970).

[659] E. Barouch, B.M. McCoy and M. Dresden, Phys. Rev. A 2, 1075 (1970).

[660] E. Barouch and B.M. McCoy, Phys. Rev. A 3, 786 (1971).

[661] E. Barouch and B.M. McCoy, Phys. Rev. A 3, 2137 (1971)

[662] E. Barouch and B.M. McCoy, Phys. Rev. A 4, 2331 (1971).

[663] A. Osterloh, private communication.

[664] S.-J. Gu, H.-Q. Lin, and Y.-Q. Li, Phys. Rev. A 68, 042330 (2003).

[665] J. Vidal, G. Palacios, and R. Mosseri, Phys. Rev. A 69, 022107 (2004).

[666] J. Vidal, R. Mosseri, and J. Dukelsky, Phys. Rev. A 69, 054101 (2004).

[667] S. Dusuel and J. Vidal, Phys. Rev. Lett. 93, 237204 (2004).

[668] T. Stauber and F. Guinea, Phys. Rev. A 70, 022313 (2004)

[669] F.C. Alcarez, A. Saguia, and M.S. Sarandy, Phys. Rev. A 70, 032333 (2004).

[670] M.-F. Yang, Phys. Rev. A 71, 030302(R) (2005).

[671] O.F. Syljuasen, Phys. Rev. A 68, 060301(R) (2003).

[672] L.-A. Wu, M.S. Sarandy, and D.A. Lidar, Phys. Rev. Lett. 93, 250404 (2004).

[673] J.I. Latorre, E. Rico, and G. Vidal, Quantum Inf. Comput. 4, 48 (2004).

[674] H. Fan, V. Korepin, and V. Roychowdhury, Phys. Rev. Lett. 93, 227203 (2004).

[675] A.R. Its, B.-Q. Jin, and V.E. Korepin, J. Phys. A: Math. Gen. 38, 2975 (2005).

[676] C. Wellard and R. Orús, Phys. Rev. A 70, 062318 (2004).

[677] J.I. Latorre, R. Orús, and J. Vidal, Phys. Rev. A 71, 064101 (2005).

[678] M.B. Plenio, J. Eisert, J. Dreissig, and M. Cramer, Phys. Rev. Lett. 94, 060503 (2005).

[679] M. Cramer, J. Eisert, M.B. Plenio, and J. Dreissig, Phys. Rev. A 73, 012309 (2006).

[680] M.M. Wolf, Phys. Rev. Lett. 96, 010404 (2006).

[681] D. Gioev and I. Klich, Phys. Rev. Lett. 96, 100503 (2006). 
[682] A. Riera and J.I. Latorre, quant-ph/0605112.

[683] W. Dür, L. Hartmann, M. Hein, M. Lewenstein, and H.-J. Briegel, Phys. Rev. Lett. 94, 097203 (2005).

[684] J.S. Bell, Physics 1, 195 (1964).

[685] S. Popescu and D. Rohrlich, Phys. Lett. A 166, 293 (1992).

[686] A. Sen(De), U. Sen, and M. Żukowski, Phys. Rev. A 68, 062306 (2003).

[687] A. Sen(De), U. Sen, and M. Żukowski, Phys. Rev. A 68, 032309 (2003).

[688] D. Aharonov, Phys. Rev. A 62, 062311 (2000).

[689] S. Scheel, J. Eisert, P.L. Knight, and M.B. Plenio, J. Mod. Opt. 50, 881 (2003).

690] M.B. Plenio, S.F. Huelga, A. Beige, and P.L. Knight, Phys. Rev. A 59, 2468 (1999).

[691] S. Bose, P.L. Knight, M.B. Plenio, and V. Vedral, Phys. Rev. Lett. 83, 5158 (1999).

[692] A. Beige, S. Bose, D. Braun, S. F. Huelga, P. L. Knight, M. B. Plenio, V. Vedral, J. Mod. Opt. 47, $2583(2000)$

[693] P. Horodecki, Phys. Rev. A 63, 022108 (2001).

694] M.B. Plenio and S.F. Huelga, Phys. Rev. Lett. 88, 197901 (2002).

[695] D. Braun, Phys. Rev. Lett. 89, 277901 (2002).

[696] X.X. Yi, C.S. Yu, L. Zhou, and H.S. Song, Phys. Rev. A 68, 052304 (2003).

[697] A.R. Its, A.G. Izergin, V.E. Korepin, and N.A. Slavnov, Phys. Rev. Lett. 70, 1704 (1993).

[698] H.G. Vaidya and C.A. Tracy, Phys. Lett. 68A, 378 (1978).

[699] J.H.H. Perk, Phys. Lett. 79A, 1 (1980).

[700] B.M. McCoy, J.H.H. Perk, and R.E. Shrock, Nucl. Phys. B 220, 35 (1983).

[701] B.M. McCoy, J.H.H. Perk, and R.E. Shrock, Nucl. Phys. B 220, 269 (1983).

[702] K. Sengupta, S. Powell, and S. Sachdev, Phys. Rev. A 69, 053616 (2004).

[703] A. Sen(De), U. Sen, and M. Lewenstein, Phys. Rev. A 70, 060304(R) (2004).

[704] J. Eisert, M.B. Plenio, S. Bose, and J. Hartley, Phys. Rev. Lett. 93, 190402 (2004).

[705] C.P. Williams and S.H. Clearwater, Explorations in Quantum Computing (Springer, New York, 1997).

[706] M.B. Plenio and P.L. Knight, Phys. Rev. A 53, 2986 (1996).

[707] A. Garg, Phys. Rev. Lett. 77, 964 (1996).

[708] D.J. Wineland, C. Monroe, W.M. Itano, B.E. King, D. Leibfried, D.M. Meekhof, C. Myatt, and C. Wood, Fortschr. Phys. 46, 363 (1998).

[709] D.F.V. James, Phys. Rev. Lett. 81, 317 (1998).

[710] J.F. Poyatos, J.I. Cirac, and P. Zoller, Phys. Rev. Lett. 81, 1322 (1998).

[711] B.E. King, C.S. Wood, C.J. Myatt, Q.A. Turchette, D. Leibfried, W.M. Itano, C. Monroe, and D.J. Wineland, Phys. Rev. Lett. 81, 1525 (1998).

[712] C. Monroe, D.M. Meekhof, B.E. King, W.M. Itano, and D.J. Wineland, Phys. Rev. Lett. 75, $4714(1995)$

[713] S. Gulde, M. Riebe, G.P.T. Lancaster, C. Becher, J. Eschner, H. Häffner, F. Schmidt-Kaler, I.L. Chuang, and R. Blatt, Nature 421, 48 (2003).

[714] F. Schmidt-Kaler, H. Häffner, M. Riebe, S. Gulde, G.P.T. Lancaster, T. Deuschle, C. Becher, C.F. Roos, J. Eschner, and R. Blatt, Nature 422, 404 (2003).

[715] H. Haeffner, W. Haensel, C.F. Roos, J. Benhelm, D. Chek-al-kar, M. Chwalla, T. Koerber, U.D. Rapol, M. Riebe, P.O. Schmidt, C. Becher, O. Gühne, W. Dür, and R. Blatt, Nature 438, 643 (2005).

[716] D. J. Wineland, D. Leibfried, M.D. Barrett, A. Ben-Kish, J.C. Bergquist, R.B. Blakestad, J.J. Bollinger, J. Britton, J. Chiaverini, B. Demarco, D. Hume, W. M. Itano, M. Jensen, J.D. Jost, E. Knill, J. Koelemeij, C. Langer, W. Oskay, R. Ozeri, R. Reichle, T. Rosenband, T. Schaetz, P. O. Schmidt, and S. Seidelin, quant-ph/0508025, Proceedings of the International Conference on Laser Spectroscopy (ICOLS).

[717] J.K. Korbicz, O. Gühne, M. Lewenstein, H. Haeffner, C.F. Roos, and R. Blatt, Phys. Rev. A 74, 052319 (2006)

[718] G.K. Brennen, C.M. Caves, P.S. Jessen, and I.H. Deutsch, Phys. Rev. Lett. 82, 1060 (1999).

[719] W. Dür, H. J. Briegel, J. I. Cirac, and P. Zoller, Phys. Rev. A 59, 169 (1999).

[720] A. Hemmerich, Phys. Rev. A 60, 943 (1999).

[721] G.K. Brennen, I.H. Deutsch, and P.S. Jessen, Phys. Rev. A 61, 062309 (2000).

[722] D. Jaksch, J.I. Cirac, P. Zoller, S.L. Rolston, R. Côté, and M.D. Lukin, Phys. Rev. Lett. 85, $2208(2000)$.

[723] M.D. Lukin, M. Fleischhauer, R. Côté, L.M. Duan, D. Jaksch, J.I. Cirac, and P. Zoller, Phys. Rev. Lett. 87, 037901 (2001).

[724] N. Schlosser, G. Reymond, I. Protsenko, and P. Grangier, Nature 411, 1024 (2001). 
[725] E. Andersson and S. Stenholm, Opt. Commun. 188, 141 (2001).

[726] K. Eckert, J. Mompart, X.X. Yi, J. Schliemann, D. Bruß, G. Birkl, and M. Lewenstein, Phys. Rev. A 66, 042317 (2002).

[727] R. Ionicioiu and P. Zanardi, Phys. Rev. A 66, 050301(R) (2002).

[728] S.E. Sklarz, I. Friedler, D.J. Tannor, Y.B. Band, and C.J. Williams, Phys. Rev. A 66, 053620 (2002).

[729] S.C. Benjamin, Phys. Rev. Lett. 88, 017904 (2002).

[730] E. Charron, E. Tiesinga, F. Mies, and C. Williams, Phys. Rev. Lett. 88, 077901 (2002).

[731] R. Dumke, T. Mther, M. Volk, W. Ertmer, and G. Birkl, Phys. Rev. Lett. 89, 220402 (2002).

[732] L. Tian and P. Zoller, Phys. Rev. A 68, 042321 (2003).

[733] J.K. Pachos and P.L. Knight, Phys. Rev. Lett. 91, 107902 (2003).

[734] J.J. García-Ripoll and J.I. Cirac, Phys. Rev. Lett. 90, 127902 (2003).

[735] K.G.H. Vollbrecht, E. Solano, and J.I. Cirac, Phys. Rev. Lett. 93, 220502 (2004).

[736] V. Murg and J.I. Cirac, Phys. Rev. A 69, 042320 (2004).

[737] T. Calarco, U. Dorner, P.S. Julienne, C.J. Williams, and P. Zoller, Phys. Rev. A 70, 012306 (2004).

[738] K. Christandl, G.P. Lafyatis, S.-C. Lee, and J.-F. Lee, Phys. Rev. A 70, 032302 (2004).

[739] A. Derevianko and C.C. Cannon, Phys. Rev. A 70, 062319 (2004).

[740] J. Vala, A.V. Thapliyal, S. Myrgen, U. Vazirani, D.S. Weiss, and K.B. Whaley, Phys. Rev. A 71, 032324 (2005).

[741] C. Lee and E.A. Ostrovskaya, Phys. Rev. A 72, 062321 (2005).

[742] A. Serafini, S. Mancini, and S. Bose, Phys. Rev. Lett. 96, 010503 (2006).

[743] R. Raussendorf and H.-J. Briegel, Phys. Rev. Lett. 86, 5188 (2001).

[744] A. Kay, J.K. Pachos, and C.S. Adams, Phys. Rev. A 73, 022310 (2006)

745] S. Clark, C. Moura-Alves, and D. Jaksch, New J. Phys. 7, 124 (2005).

[746] M. Christandl, N. Datta, T.C. Dorlas, A. Ekert, A. Kay, and A.J. Landahl, Phys. Rev. A 71, $032312(2005)$.

[747] R. Raussendorf and H.-J. Briegel, Phys. Rev. Lett. 86, 910 (2001).

[748] N. Deo, Graph Theory with Applications to Engineering and Computer Science (Prentice Hall, 1974)

[749] R. Raussendorf, D.E. Browne, and H.-J. Briegel, J. Mod. Opt. 49, 1299 (2002).

[750] R. Raussendorf, D.E. Browne, and H.-J. Briegel, Phys. Rev. A 68, 022312 (2003).

[751] D. Gottesman, Ph.D. thesis, California Institute of Technology, USA, 1997 (quant$\mathrm{ph} / 9705052)$

[752] M.A. Nielsen, Phys. Lett. A 308, 96 (2003).

[753] D.W. Leung, quant-ph/0111122.

[754] D.W. Leung, Int. Jour. Quant. Inf. 2, 33 (2004) (quant-ph/0310189).

[755] M. Hein, J. Eisert, and H.-J. Briegel, Phys. Rev. A 69, 062311 (2004)

[756] M. Asano, N. Tateda, and C. Ishii, Phys. Rev. A 70, 022315 (2004).

[757] M. Van den Nest, J. Dehaene, and B. De Moor, Phys. Rev A 70, 032323 (2004).

[758] M. Van den Nest, J. Dehaene, and B. De Moor, Phys. Rev. A 70, 034302 (2004).

[759] P. Aliferis, D.W. Leung, Phys. Rev. A 70, 062314 (2004).

[760] H. Aschauer, W. Dür, and H.-J. Briegel, Phys. Rev. A 71, 012319 (2005).

[761] A.M. Childs, D.W. Leung, and M.A. Nielsen, Phys. Rev. A 71, 032318 (2005).

[762] M. Borhani and D. Loss, Phys. Rev. A 71, 034308 (2005).

[763] P. Walther, M. Aspelmeyer, K.J. Resch, and A. Zeilinger, Phys. Rev. Lett 95, 020403 (2005).

[764] L.-M. Duan and R. Raussendorf, Phys. Rev. Lett. 95, 080503 (2005).

[765] E. Hostens, J. Dehaene, and B. De Moor, Phys. Rev. A 71, 042315 (2005).

[766] M.A. Nielsen and C.M. Dawson, Phys. Rev. A 71, 042323 (2005).

[767] M.A. Nielsen, quant-ph/0504097.

[768] M. Van den Nest, J. Dehaene, and B. De Moor, Phys. Rev. A 71, 062323 (2005).

[769] M.S. Tame, M. Paternostro, M.S. Kim, and V. Vedral, Phys. Rev. A 72, 012319 (2005).

[770] M. Van den Nest, J. Dehaene, and B. De Moor, Phys. Rev. A 72, 014307 (2005).

[771] Q. Chen, J. Cheng, K.-L. Wang, and J. Du, Phys. Rev. A 73, 012303 (2006).

[772] M.S. Tame, M. Paternostro, M.S. Kim, and V. Vedral, Phys. Rev. A 73, 022309 (2006).

[773] S. Anders and H.-J. Briegel, Phys. Rev. A 73, 022334 (2006).

774] P. Aliferis and D.W. Leung, Phys. Rev. A 73, 032308 (2006)

[775] For the first experiment see P.O. Schmidt, S. Hensler, J. Werner, A. Griesmaier, A. Görlitz, and T. Pfau, Phys. Rev. Lett. 91, 193201 (2003).

[776] M. Pons, V. Ahufinger, C. Wunderlich, A. Sanpera, S. Braungardt, A. Sen(De), U. Sen, and 
M. Lewenstein, to appear in Phys. Rev. Lett. (quant-ph/0607016)

[777] H. Cho, T.D. Ladd, J. Baugh, D.G. Cory, and C. Ramanathan, Phys. Rev. B 72, 054427 (2005).

[778] D.J. Amit, Modeling Brain Function: The World of Attractor Neural Networks (Cambridge University Press, Cambridge, 1992)

[779] P.W. Shor, Phys. Rev. A 52, R2493 (1995)

[780] A.M. Steane, Proc. R. Soc. London A, 452, 2551 (1996).

[781] A. Sen(De), U. Sen, V. Ahufinger, H.-J. Briegel, A. Sanpera, and M. Lewenstein, to appear in Phys. Rev. A (quant-ph/0507172).

[782] A. Sen(De), U. Sen, M. Lewenstein, M. Pons, V. Ahufinger, A. Sanpera, quant-ph/0508018.

[783] S. Braungardt, A. Sen(De), U. Sen, and M. Lewenstein, quant-ph/0607053.

[784] S. Popescu, Phys. Rev. Lett. 72, 797 (1994). 